Supporting Information

\title{
NHC-Catalyzed Radical Trifluoromethylation Enabled by Togni Reagent
}

Bei Zhang, Qiupeng Peng, Donghui Guo and Jian Wang*

School of Pharmaceutical Sciences, Key Laboratory of Bioorganic Phosphorous

Chemistry \& Chemical Biology (Ministry of Education),

Tsinghua University, Beijing, 100084, China.

E-mail: wangjian2012@tsinghua.edu.cn

\section{Contents}

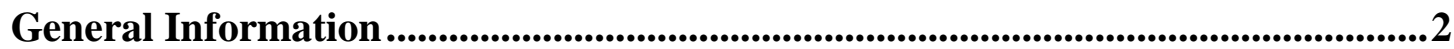

General Procedure for preparing trifluoromethylated styrenes ...............................2

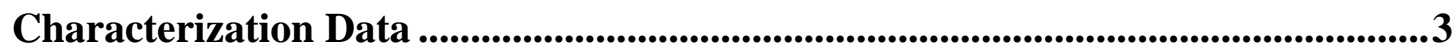

The reaction of 1a with $2 \mathrm{a}$ at 1 mmol Scale ......................................................21

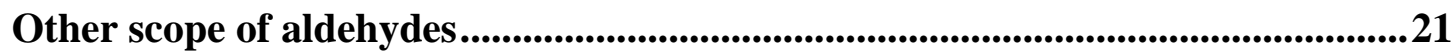

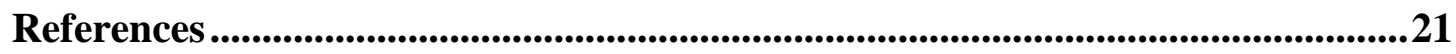

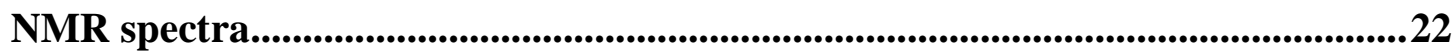




\section{General Information}

Chemicals were purchased from commercial suppliers and used as received. Solvents were dried on alumina columns using a solvent dispensing system. Thin-layer chromatography (TLC) was conducted on plates (GF254) supplied by Yantai Chemicals (China) and visualized using a combination of UV, anisaldehyde, iodine, and potassium permanganate staining. ${ }^{1} \mathrm{H} \mathrm{NMR},{ }^{13} \mathrm{C} \mathrm{NMR},{ }^{19} \mathrm{~F}$ NMR, spectra were recorded on a Bruker ACF400 (400 MHz) spectrometer. Chemical shifts were reported in parts per million (ppm), and the residual solvent peak was used as an internal reference: proton (chloroform $\delta 7.26$, menthol $\delta 3.31$ ), carbon (chloroform $\delta 77.16$ ) or tetramethylsilane (TMS $\delta 0.00$ ) was used as a reference. Multiplicity was indicated as follows: s (singlet), $d$ (doublet), $\mathrm{t}$ (triplet), $\mathrm{q}$ (quartet), $\mathrm{m}$ (multiplet), dd (doublet of doublet), bs (broad singlet). Coupling constants were reported in Hertz (Hz). All high resolution mass spectra were obtained from the Tsinghua University Mass Spectrometry Facility. Flash chromatography separations were performed on Silica gel (300-400 mesh) supplied by Tsingdao Haiyang Chemicals (China). Aromatic styrenes were prepared by literatures ${ }^{1}$ or commercial available.

\section{General Procedure for preparing trifluoromethylated styrenes}<smiles>[R]C=C[CH-][CH]C([R])=O</smiles><smiles></smiles>

To a flame-dried Schlenk reaction tube equipped with a magnetic stir bar, was added the pre-catalyst $\mathbf{C 3}$ (16.6 mg, $0.04 \mathrm{mmol}$ ), $\mathrm{Cs}_{2} \mathrm{CO}_{3}$ (26.1 mg, $0.08 \mathrm{mmol}$ ) and 3a (132.0 $\mathrm{mg}, 0.4 \mathrm{mmol})$. The Schlenk tube was closed with a septum, evacuated and refilled with argon atmosphere. $1(0.24 \mathrm{mmol})$ and $\mathbf{2}(0.20 \mathrm{mmol})$ in DCM $(2 \mathrm{~mL})$ was added via a syringe. Water $(40 \mu \mathrm{L})$ was added subsequently. The mixture was then stirred at $25^{\circ} \mathrm{C}$ and monitored by TLC until 2 was consumed completely. The mixture was concentrated under reduced pressure and purified by flash column chromatography (hexane : EA, 20:1 to 3:1) to afford the trifluoromethylated product $\mathbf{4}$ or $\mathbf{5}$. 


\section{Characterization Data}<smiles>O=C(c1ccc(Cl)cc1)C(CC(F)(F)F)c1ccccc1</smiles>

4a: $52.5 \mathrm{mg}, 84 \%$ yield, white solid, m.p.: $67-71{ }^{\circ} \mathrm{C}, 6 \mathrm{~h}$, hexane: $\mathrm{EA}=20: 1, \mathrm{R}_{\mathrm{f}}=0.6$. ${ }^{1}$ H NMR (400 MHz, Chloroform- $\left.d\right) \delta 7.92(\mathrm{~d}, J=8.7 \mathrm{~Hz}, 2 \mathrm{H}), 7.40(\mathrm{~d}, J=8.7 \mathrm{~Hz}$, $2 \mathrm{H}), 7.37-7.24(\mathrm{~m}, 5 \mathrm{H}), 4.86(\mathrm{dd}, J=7.7,5.4 \mathrm{~Hz}, 1 \mathrm{H}), 3.42-3.12(\mathrm{~m}, 1 \mathrm{H})$, 2.49-2.63 (m, 1H).

${ }^{19}$ F NMR $(376 \mathrm{MHz}$, Chloroform- $d) \delta-64.60$.

${ }^{13}$ C NMR (100 MHz, Chloroform- $d$ ) $\delta$ 195.5, 139.9, 137.1, 134.0, 130.2, 129.5, 129.0, 128.0, 128.0, 126.3 (q, $J=277.3 \mathrm{~Hz}), 47.3(\mathrm{q}, J=2.4 \mathrm{~Hz}), 37.3(\mathrm{q}, J=28.3 \mathrm{~Hz})$.

IR (neat, ATR) 2988, 2901, 1663, 1600, 1590, 1510, 1493, 1136, 1070, 752, 698 $\mathrm{cm}^{-1}$.

HRMS (ESI): $m / z$ : calculated for $\mathrm{C}_{16} \mathrm{H}_{13} \mathrm{ClF}_{3} \mathrm{O}^{+}[\mathrm{M}+\mathrm{H}]^{+}$313.0602, found: 313.0603 .<smiles>O=C(c1ccc(Cl)cc1)C(CC(F)(F)F)c1ccc(Cl)cc1</smiles>

4b: $58.1 \mathrm{mg}, 84 \%$ yield, colorless oil, $12 \mathrm{~h}$, hexane: $\mathrm{EA}=20: 1, \mathrm{R}_{\mathrm{f}}=0.6$.

${ }^{1}$ H NMR (400 MHz, Chloroform- $d$ ) $\delta 7.89(\mathrm{~d}, J=8.7 \mathrm{~Hz}, 2 \mathrm{H}), 7.41(\mathrm{~d}, J=8.7 \mathrm{~Hz}, 2 \mathrm{H})$, $7.32(\mathrm{~d}, J=8.6 \mathrm{~Hz}, 2 \mathrm{H}), 7.24(\mathrm{~d}, J=8.5 \mathrm{~Hz}, 2 \mathrm{H}), 4.84(\mathrm{t}, J=6.6 \mathrm{~Hz}, 1 \mathrm{H}), 3.42-3.11(\mathrm{~m}$, $1 \mathrm{H}), 2.49-2.62(\mathrm{~m}, 1 \mathrm{H})$.

${ }^{19}$ F NMR (376 MHz, Chloroform- $d$ ) $\delta-64.45$.

${ }^{13}$ C NMR (100 MHz, Chloroform- $d$ ) $\delta$ 195.2, 140.2, 135.5, 134.1, 133.6, 130.2, 129.7, $129.3,129.1,126.2(\mathrm{q}, J=277.2 \mathrm{~Hz}), 46.6(\mathrm{q}, J=2.2 \mathrm{~Hz}), 37.2(\mathrm{q}, J=28.4 \mathrm{~Hz})$.

IR (neat, ATR) 3066, 2956, 1685, 1590, 1571, 1490, 1434, 1256, 1092, 797, 745 $\mathrm{cm}^{-1}$.

HRMS (ESI): $m / z$ : calculated for $\mathrm{C}_{16} \mathrm{H}_{12} \mathrm{C}_{12} \mathrm{~F}_{3} \mathrm{O}^{+}[\mathrm{M}+\mathrm{H}]^{+}$347.0212, found: 347.0209 .<smiles>N#Cc1ccc(C(CC(F)(F)F)C(=O)c2ccc(Cl)cc2)cc1</smiles>

4c: $60.5 \mathrm{mg}, 90 \%$ yield, colorless oil, $12 \mathrm{~h}$, hexane: $\mathrm{EA}=20: 1, \mathrm{R}_{\mathrm{f}}=0.3$. 
${ }^{1}$ H NMR (400 MHz, Chloroform- $\left.d\right) \delta 7.88(\mathrm{~d}, J=8.6 \mathrm{~Hz}, 2 \mathrm{H}), 7.65(\mathrm{~d}, J=8.3 \mathrm{~Hz}$, $2 \mathrm{H}), 7.42-7.46(\mathrm{~m}, 4 \mathrm{H}), 4.93(\mathrm{t}, J=6.7 \mathrm{~Hz}, 1 \mathrm{H}), 3.38-3.13(\mathrm{~m}, 1 \mathrm{H}), 2.53-2.67(\mathrm{~m}$, 1H).

${ }^{19}$ F NMR (376 MHz, Chloroform- $d$ ) $\delta-64.34$.

${ }^{13}$ C NMR (100 MHz, Chloroform- $d$ ) $\delta$ 194.6, 142.1, 140.6, 133.4, 133.2, 130.2, 129.3, $128.9,118.1,112.3,126.0(\mathrm{q}, J=277.3 \mathrm{~Hz}), 47.1(\mathrm{q}, J=2.2 \mathrm{~Hz}), 37.1(\mathrm{q}, J=28.8$ $\mathrm{Hz}$ ).

IR (neat, ATR) 3067, 2958, 2231, 1686, 1607, 1588, 1504, 1489, 1257, 1094, 798, $780,760,732 \mathrm{~cm}^{-1}$.

HRMS (ESI): $m / z$ : calculated for $\mathrm{C}_{17} \mathrm{H}_{12} \mathrm{ClF}_{3} \mathrm{NO}^{+}[\mathrm{M}+\mathrm{H}]^{+}$338.0554, found: 338.0554.<smiles>COc1ccc(C(CC(F)(F)F)C(=O)c2ccc(Cl)cc2)cc1</smiles>

4d: $51.8 \mathrm{mg}, 76 \%$ yield, colorless oil, $12 \mathrm{~h}$, hexane: $\mathrm{EA}=20: 1, \mathrm{R}_{\mathrm{f}}=0.4$.

${ }^{1} \mathbf{H}$ NMR $(400 \mathrm{MHz}$, Chloroform- $d$ ) $\delta 7.91(\mathrm{~d}, J=8.6 \mathrm{~Hz}, 2 \mathrm{H}), 7.39(\mathrm{~d}, J=8.6 \mathrm{~Hz}, 2 \mathrm{H})$, $7.21(\mathrm{~d}, J=8.7 \mathrm{~Hz}, 2 \mathrm{H}), 6.86(\mathrm{~d}, J=8.7 \mathrm{~Hz}, 2 \mathrm{H}), 5.01-4.73(\mathrm{~m}, 1 \mathrm{H}), 3.77(\mathrm{~s}, 3 \mathrm{H}), 3.44$ $-3.12(\mathrm{~m}, 1 \mathrm{H}), 2.75-2.37(\mathrm{~m}, 1 \mathrm{H})$.

${ }^{19}$ F NMR (376 MHz, Chloroform- $d$ ) $\delta-64.50$.

${ }^{13}$ C NMR (100 MHz, Chloroform- $d$ ) $\delta$ 195.7, 159.2, 139.8, 134.0, 130.2, 129.1, 129.0, $128.9,126.4$ (q, $J=277.3 \mathrm{~Hz}), 114.8,55.2,46.5(\mathrm{q}, J=1.9 \mathrm{~Hz}), 37.3(\mathrm{q}, J=28.1 \mathrm{~Hz})$. IR (neat, ATR) 2958, 2839, 1685, 1610, 1589, 1571, 1510, 1249, 1093, 786, 765 $\mathrm{cm}^{-1}$.

HRMS (ESI): $m / z$ : calculated for $\mathrm{C}_{17} \mathrm{H}_{15} \mathrm{ClF}_{3} \mathrm{O}_{2}{ }^{+}[\mathrm{M}+\mathrm{H}]^{+}$343.0707, found: 343.0710 .<smiles>Cc1ccc(C(CC(F)(F)F)C(=O)c2ccc(Cl)cc2)cc1</smiles>

4e: $54.9 \mathrm{mg}, 84 \%$ yield, colorless oil, $12 \mathrm{~h}$, hexane: $\mathrm{EA}=20: 1, \mathrm{R}_{\mathrm{f}}=0.6$.

${ }^{1} \mathbf{H}$ NMR (400 MHz, Chloroform- $d$ ) $\delta 7.91(\mathrm{~d}, J=8.7 \mathrm{~Hz}, 2 \mathrm{H}), 7.39(\mathrm{~d}, J=8.7 \mathrm{~Hz}, 2 \mathrm{H})$, $7.17(\mathrm{~m}, 4 \mathrm{H}), 4.82(\mathrm{dd}, J=7.5,5.6 \mathrm{~Hz}, 1 \mathrm{H}), 3.44-3.15(\mathrm{~m}, 1 \mathrm{H}), 2.63-2.44(\mathrm{~m}, 1 \mathrm{H})$, $2.31(\mathrm{~s}, 3 \mathrm{H})$.

${ }^{19}$ F NMR (376 MHz, Chloroform- $d$ ) $\delta-64.57$.

${ }^{13}$ C NMR (100 MHz, Chloroform- $d$ ) $\delta$ 195.6, 139.8, 137.8, 134.0, 134.0, 130.2, 130.1, 129.0, 127.8, $126.4(\mathrm{q}, J=266 \mathrm{~Hz}), 46.9(\mathrm{q}, J=2.1 \mathrm{~Hz}), 37.3(\mathrm{q}, J=28.2 \mathrm{~Hz}), 21.0$. 
IR (neat, ATR) 2958, 2830, 1685, 1590, 1571, 1512, 1489, 1257, 1094, 846, 816, 793, $767 \mathrm{~cm}^{-1}$.

HRMS (ESI): $m / z$ : calculated for $\mathrm{C}_{17} \mathrm{H}_{14} \mathrm{ClF}_{3} \mathrm{NaO}^{+}[\mathrm{M}+\mathrm{Na}]^{+}$349.0577, found: 349.0579 .<smiles>O=C(c1ccc(Cl)cc1)C(CC(F)(F)F)c1cccc(Cl)c1</smiles>

4f: $55.5 \mathrm{mg}, 80 \%$ yield, colorless oil, $12 \mathrm{~h}$, hexane: $\mathrm{EA}=20: 1, \mathrm{R}_{\mathrm{f}}=0.6$.

${ }^{1}$ H NMR (400 MHz, Chloroform- $d$ ) $\delta 7.91(\mathrm{dd}, J=8.9,2.1 \mathrm{~Hz}, 2 \mathrm{H}), 7.42(\mathrm{dd}, J=8.9$, $2.0 \mathrm{~Hz}, 2 \mathrm{H}), 7.34-7.24(\mathrm{~m}, 3 \mathrm{H}), 7.23-7.16(\mathrm{~m}, 1 \mathrm{H}), 4.84(\mathrm{dd}, J=7.2,6.0 \mathrm{~Hz}, 1 \mathrm{H})$, $3.50-2.98(\mathrm{~m}, 1 \mathrm{H}), 2.71-2.27(\mathrm{~m}, 1 \mathrm{H})$.

${ }^{19}$ F NMR (376 MHz, Chloroform- $d$ ) $\delta$-64.54.

${ }^{13}$ C NMR (100 MHz, Chloroform- $d$ ) $\delta$ 195.0, 140.3, 138.9, 135.3, 133.6, 130.7, 130.2, $129.2,128.4,128.1,126.2,126.1(\mathrm{q}, J=277.3 \mathrm{~Hz}), 46.8(\mathrm{q}, J=2.4 \mathrm{~Hz}), 37.2(\mathrm{q}, J=$ $28.5 \mathrm{~Hz})$

IR (neat, ATR) 2958, 2830, 1685, 1590, 1571, 1512, 1489, 1257, 1094, 846, 816, 793, $767 \mathrm{~cm}^{-1}$.

HRMS (ESI): $m / z$ : calculated for $\mathrm{C}_{16} \mathrm{H}_{12} \mathrm{C}_{12} \mathrm{~F}_{3} \mathrm{O}^{+}[\mathrm{M}+\mathrm{H}]^{+}$347.0212, found: 347.0216.<smiles>Cc1ccccc1C(CC(F)(F)F)C(=O)c1ccc(Cl)cc1</smiles>

4g: $52.1 \mathrm{mg}, 80 \%$ yield, colorless oil, $12 \mathrm{~h}$, hexane: $\mathrm{EA}=20: 1, \mathrm{R}_{\mathrm{f}}=0.6$.

${ }^{1} \mathbf{H}$ NMR (400 MHz, Chloroform- $d$ ) $\delta 7.84-7.78(\mathrm{~m}, 2 \mathrm{H}), 7.47-7.31$ (m, 2H), 7.30 $7.24(\mathrm{~m}, 1 \mathrm{H}), 7.10-7.21(\mathrm{~m}, 2 \mathrm{H}), 7.04(\mathrm{dd}, J=7.7,1.3 \mathrm{~Hz}, 1 \mathrm{H}), 4.99(\mathrm{dd}, J=8.4,4.0 \mathrm{~Hz}$, $1 \mathrm{H}), 3.55-3.27(\mathrm{~m}, 1 \mathrm{H}), 2.57(\mathrm{~s}, 3 \mathrm{H}), 2.32-2.41(\mathrm{~m}, 1 \mathrm{H})$.

${ }^{19}$ F NMR (376 MHz, Chloroform- $d$ ) $\delta-65.16$.

${ }^{13}$ C NMR (100 MHz, Chloroform- $d$ ) $\delta$ 196.1, 139.7, 135.7, 135.0, 134.3, 131.7, 129.9, $129.0,128.0,127.1,127.0,126.3(\mathrm{q}, J=277.3 \mathrm{~Hz}), 43.6(\mathrm{q}, J=2.6 \mathrm{~Hz}), 36.5(\mathrm{q}, J=$ $28.4 \mathrm{~Hz}), 19.5$.

IR (neat, ATR) 2958, 2850, 1688, 1590, 1572, 1489, 1462, 1257, 1090, 841, 797, 756, $728 \mathrm{~cm}^{-1}$.

HRMS (ESI): $m / z$ : calculated for $\mathrm{C}_{17} \mathrm{H}_{14} \mathrm{ClF}_{3} \mathrm{NaO}^{+}[\mathrm{M}+\mathrm{Na}]^{+}$349.0577, found: 349.0574 . 
<smiles>O=C(c1ccc(Cl)cc1)C(CC(F)(F)F)c1ccccc1Br</smiles>

4h: $61.6 \mathrm{mg}$, 79\% yield, colorless oil, $12 \mathrm{~h}$, hexane: $\mathrm{EA}=20: 1, \mathrm{R}_{\mathrm{f}}=0.6$.

${ }^{1}$ H NMR (400 MHz, Chloroform- $d$ ) $\delta 7.97-7.89(\mathrm{~m}, 2 \mathrm{H}), 7.69-7.56(\mathrm{~m}, 1 \mathrm{H}), 7.48-$ $7.38(\mathrm{~m}, 2 \mathrm{H}), 7.27-7.20(\mathrm{~m}, 1 \mathrm{H}), 7.19-7.10(\mathrm{~m}, 2 \mathrm{H}), 5.39$ (dd, $J=8.4,4.4 \mathrm{~Hz}, 1 \mathrm{H})$, $3.48-3.00(\mathrm{~m}, 1 \mathrm{H}), 2.38-2.51(\mathrm{~m}, 1 \mathrm{H})$.

${ }^{19}$ F NMR (376 MHz, Chloroform- $d$ ) $\delta-64.75$.

${ }^{13}$ C NMR (100 MHz, Chloroform- $d$ ) $\delta$ 195.3, 140.2, 136.5, 134.0, 133.7, 130.2, 129.7, $129.1,128.7,128.5,126.0(\mathrm{q}, J=277.5 \mathrm{~Hz}), 124.1,46.0(\mathrm{q}, J=2.2 \mathrm{~Hz}), 36.4(\mathrm{q}, J=$ $28.8 \mathrm{~Hz})$.

IR (neat, ATR) 3010, 2959, 1687, 1590, 1571, 1489, 1471, 1256, 1094, 754, 741, 722, $688 \mathrm{~cm}^{-1}$.

HRMS (ESI): $m / z$ : calculated for $\mathrm{C}_{16} \mathrm{H}_{12} \mathrm{BrClF}_{3} \mathrm{O}^{+}[\mathrm{M}+\mathrm{H}]^{+}$390.9707, found: 390.9705 .<smiles>O=C(c1ccc(Cl)cc1)C(CC(F)(F)F)c1ccccc1Cl</smiles>

4i: $57.4 \mathrm{mg}, 83 \%$ yield, colorless oil, $12 \mathrm{~h}$, hexane: $\mathrm{EA}=20: 1, \mathrm{R}_{\mathrm{f}}=0.6$.

${ }^{1}$ H NMR (400 MHz, Chloroform- $d$ ) $\delta 7.98-7.89$ (m, 2H), $7.47(\mathrm{dd}, J=6.3,1.5 \mathrm{~Hz}$, $1 \mathrm{H}), 7.43-7.35(\mathrm{~m}, 2 \mathrm{H}), 7.27-7.07(\mathrm{~m}, 3 \mathrm{H}), 5.40(\mathrm{dd}, J=7.8,5.1 \mathrm{~Hz}, 1 \mathrm{H}), 3.43-$ $3.19(\mathrm{~m}, 1 \mathrm{H}), 2.62-2.37(\mathrm{~m}, 1 \mathrm{H})$.

${ }^{19}$ F NMR (376 MHz, Chloroform- $d$ ) $\delta-64.88$.

${ }^{13}$ C NMR (100 MHz, Chloroform- $d$ ) $\delta$ 195.3, 140.2, 134.9, 133.7, 133.3, 130.5, 130.1, $129.4,129.1,128.6,127.8,126.1$ (q, $J=277.4 \mathrm{~Hz}), 43.2$ (q, $J=2.6 \mathrm{~Hz}), 36.4$ (q, $J=$ $28.9 \mathrm{~Hz})$.

IR (neat, ATR) 3010, 2961, 1688, 1590, 1571, 1489, 1476, 1256, 1094, 755, 746, 726, $697 \mathrm{~cm}^{-1}$.

HRMS (ESI): $m / z$ : calculated for $\mathrm{C}_{16} \mathrm{H}_{12} \mathrm{Cl}_{2} \mathrm{~F}_{3} \mathrm{O}^{+}[\mathrm{M}+\mathrm{H}]^{+}$347.0212, found: 347.0216 .<smiles>O=C(c1ccc(Cl)cc1)C(CC(F)(F)F)c1cccc2ccccc12</smiles>

4j: $60.1 \mathrm{mg}, 83 \%$ yield, colorless oil, $12 \mathrm{~h}$, hexane: $\mathrm{EA}=20: 1, \mathrm{R}_{\mathrm{f}}=0.5$.

${ }^{1}$ H NMR (400 MHz, Chloroform- $d$ ) $\delta 8.00-7.94(\mathrm{~m}, 2 \mathrm{H}), 7.88-7.71(\mathrm{~m}, 4 \mathrm{H}), 7.59-$ $7.46(\mathrm{~m}, 2 \mathrm{H}), 7.45-7.31(\mathrm{~m}, 3 \mathrm{H}), 5.03(\mathrm{dd}, J=7.4,5.6 \mathrm{~Hz}, 1 \mathrm{H}), 3.81-3.21(\mathrm{~m}, 1 \mathrm{H})$, 
2.59-2.72 (m, 1H).

${ }^{19}$ F NMR (376 MHz, Chloroform- $d$ ) $\delta-64.51$.

${ }^{13}$ C NMR (100 MHz, Chloroform- $d$ ) $\delta$ 195.4, 139.9, 134.4, 133.9, 133.6, 132.7, 130.3, $129.5,129.0,127.8,127.7,127.2,126.7,126.5,125.4,126.4$ (q, $J=277.4 \mathrm{~Hz}), 47.4$ (q, $J=2.3 \mathrm{~Hz}), 37.3(\mathrm{q}, J=28.3 \mathrm{~Hz})$.

IR (neat, ATR) 3060, 2959, 1683, 1590, 1571, 1508, 1488, 1255, 1093, 842, 817, 798, $746 \mathrm{~cm}^{-1}$.

HRMS (ESI): $m / z$ : calculated for $\mathrm{C}_{20} \mathrm{H}_{15} \mathrm{ClF}_{3} \mathrm{O}^{+}[\mathrm{M}+\mathrm{H}]^{+}$363.0758, found: 363.0754 .<smiles>O=C(c1ccc(Cl)cc1)C(CC(F)(F)F)c1ccc2ncccc2c1</smiles>

4k: $64.7 \mathrm{mg}, 89 \%$ yield, colorless oil, $12 \mathrm{~h}$, hexane: $\mathrm{EA}=10: 1, \mathrm{R}_{\mathrm{f}}=0.4$.

${ }^{1}$ H NMR (400 MHz, Chloroform- $d$ ) $\delta 8.92(\mathrm{dd}, J=4.2,1.6 \mathrm{~Hz}, 1 \mathrm{H}), 8.22-8.03(\mathrm{~m}$, $2 \mathrm{H}), 7.98-7.88(\mathrm{~m}, 2 \mathrm{H}), 7.77-7.59(\mathrm{~m}, 2 \mathrm{H}), 7.51-7.36(\mathrm{~m}, 3 \mathrm{H}), 5.16-4.85(\mathrm{~m}, 1 \mathrm{H})$, $3.54-3.21(\mathrm{~m}, 1 \mathrm{H}), 2.61-2.74(\mathrm{~m}, 1 \mathrm{H})$.

${ }^{19}$ F NMR (376 MHz, Chloroform- $d$ ) $\delta$-64.44.

${ }^{13}$ C NMR (100 MHz, Chloroform- $d$ ) $\delta$ 195.3, 151.1, 147.6, 140.2, 135.9, 135.3, 133.7, $131.1,130.2,129.2,128.4,127.0,126.2$ (q, $J=277.2 \mathrm{~Hz}), 121.8,47.1$ (q, $J=2.4 \mathrm{~Hz}$ ), $37.4(\mathrm{q}, J=28.6 \mathrm{~Hz})$, missing one carbon.

IR (neat, ATR) 3061, 2955, 1683, 1590, 1571, 1500, 1433, 1258, 1094, 835, 800, 774, $731 \mathrm{~cm}^{-1}$.

HRMS (ESI): $m / z$ : calculated for $\mathrm{C}_{19} \mathrm{H}_{14} \mathrm{ClF}_{3} \mathrm{NO}^{+}[\mathrm{M}+\mathrm{H}]^{+}$364.0711, found: 364.0715 .<smiles>O=C(c1ccc(Cl)cc1)C(CC(F)(F)F)c1ccccn1</smiles>

4l: $43.0 \mathrm{mg}, 69 \%$ yield, colorless oil, $12 \mathrm{~h}$, hexane: $\mathrm{EA}=5: 1, \mathrm{R}_{\mathrm{f}}=0.4$.

${ }^{1}$ H NMR (400 MHz, Chloroform- $d$ ) $\delta 8.58(\mathrm{~d}, J=3.8 \mathrm{~Hz}, 1 \mathrm{H}), 8.01(\mathrm{~d}, J=8.4 \mathrm{~Hz}, 2 \mathrm{H})$, $7.66(\mathrm{t}, J=7.6 \mathrm{~Hz}, 1 \mathrm{H}), 7.41(\mathrm{~d}, J=8.4 \mathrm{~Hz}, 2 \mathrm{H}), 7.30(\mathrm{~d}, J=8.4 \mathrm{~Hz}, 1 \mathrm{H}), 7.24-7.14$ $(\mathrm{m}, 1 \mathrm{H}), 5.13(\mathrm{t}, J=6.5 \mathrm{~Hz}, 1 \mathrm{H}), 3.45-3.09(\mathrm{~m}, 1 \mathrm{H}), 2.74-2.84(\mathrm{~m}, 1 \mathrm{H})$.

${ }^{19}$ F NMR (376 MHz, Chloroform- $d$ ) $\delta-64.45$.

${ }^{13}$ C NMR (100 MHz, Chloroform- $d$ ) $\delta$ 194.8, 156.8, 150.2, 140.1, 137.4, 133.9, 130.5, 129.0, 122.8, 122.6, $50.1(\mathrm{q}, J=2.0 \mathrm{~Hz}), 35.9$ (q, $J=29.2 \mathrm{~Hz})$, missing one carbon.

IR (neat, ATR) 3088, 2988, 1693, 1590, 1571, 1471, 1437, 1280, 1136, 843, 811, 773, $748 \mathrm{~cm}^{-1}$. 
HRMS (ESI): $m / z$ : calculated for $\mathrm{C}_{15} \mathrm{H}_{12} \mathrm{ClF}_{3} \mathrm{NO}^{+}[\mathrm{M}+\mathrm{H}]^{+}$314.0554, found: 314.0557.<smiles>O=C(c1ccc(Cl)cc1)C(CC(F)(F)F)c1ccncc1</smiles>

4m: $50.1 \mathrm{mg}, 80 \%$ yield, colorless oil, $12 \mathrm{~h}$, hexane: $\mathrm{EA}=3: 1, \mathrm{R}_{\mathrm{f}}=0.6$.

${ }^{1} \mathbf{H}$ NMR (400 MHz, Chloroform- $d$ ) $\delta 8.58(\mathrm{dd}, J=4.5,1.5 \mathrm{~Hz}, 2 \mathrm{H}), 7.88(\mathrm{dd}, J=8.9$, $2.2 \mathrm{~Hz}, 2 \mathrm{H}), 7.49-7.38(\mathrm{~m}, 2 \mathrm{H}), 7.34-7.10(\mathrm{~m}, 2 \mathrm{H}), 4.85(\mathrm{t}, J=6.6 \mathrm{~Hz}, 1 \mathrm{H}), 3.46-$ $2.87(\mathrm{~m}, 1 \mathrm{H}), 2.51-2.65(\mathrm{~m} 1 \mathrm{H})$.

${ }^{19}$ F NMR (376 MHz, Chloroform- $d$ ) $\delta-64.48$.

${ }^{13}$ C NMR (100 MHz, Chloroform- $d$ ) $\delta$ 194.4, 150.8, 145.8, 140.6, 133.4, 130.1, 129.3, $126.0(\mathrm{q}, J=277.2 \mathrm{~Hz}), 123.0,46.5(\mathrm{q}, J=2.7 \mathrm{~Hz}), 36.9(\mathrm{q}, J=28.8 \mathrm{~Hz})$.

IR (neat, ATR) 3088, 2960, 1683, 1590, 1571, 1487, 1473, 1287, 1103, 787, 758, 659, $631 \mathrm{~cm}^{-1}$.

HRMS (ESI): $m / z$ : calculated for $\mathrm{C}_{15} \mathrm{H}_{12} \mathrm{ClF}_{3} \mathrm{NO}^{+}[\mathrm{M}+\mathrm{H}]^{+}$314.0554, found: 314.0550 .<smiles>O=C(c1ccc(Cl)cc1)C(CC(F)(F)F)c1ccsc1</smiles>

4n: $38.2 \mathrm{mg}, 60 \%$ yield, colorless oil, $12 \mathrm{~h}$, hexane: $\mathrm{EA}=20: 1, \mathrm{R}_{\mathrm{f}}=0.3$.

${ }^{1} \mathbf{H}$ NMR (400 MHz, Chloroform- $d$ ) $\delta 7.92(\mathrm{~d}, J=8.6 \mathrm{~Hz}, 2 \mathrm{H}), 7.42(\mathrm{~d}, J=8.6 \mathrm{~Hz}, 2 \mathrm{H})$, $7.32(\mathrm{dd}, J=5.0,3.0 \mathrm{~Hz}, 1 \mathrm{H}), 7.16(\mathrm{dd}, J=2.7,1.1 \mathrm{~Hz}, 1 \mathrm{H}), 7.01(\mathrm{dd}, J=5.0,1.1 \mathrm{~Hz}$, $1 \mathrm{H}), 5.02(\mathrm{dd}, J=7.8,5.3 \mathrm{~Hz}, 1 \mathrm{H}), 3.24-3.33(\mathrm{~m}, 1 \mathrm{H}), 2.76-2.48(\mathrm{~m}, 1 \mathrm{H})$.

${ }^{19}$ F NMR (376 MHz, Chloroform- $d$ ) $\delta-64.75$.

${ }^{13}$ C NMR (100 MHz, Chloroform- $d$ ) $\delta$ 195.2, 140.0, 136.9, 133.8, 130.1, 129.1, 127.3, 126.6, 126.2 (q, $J=267.0 \mathrm{~Hz}), 123.1,42.3(\mathrm{q}, J=2.6 \mathrm{~Hz}), 37.0(\mathrm{q}, J=28.3 \mathrm{~Hz})$.

IR (neat, ATR) 3090, 2968, 1680, 1589, 1572, 1488, 1439, 1282, 1108, 842, 781, 656, $627 \mathrm{~cm}^{-1}$.

HRMS (ESI): $m / z$ : calculated for $\mathrm{C}_{14} \mathrm{H}_{11} \mathrm{ClF}_{3} \mathrm{OS}^{+}[\mathrm{M}+\mathrm{H}]^{+}$319.0166, found: 319.0169 .<smiles>O=C(c1ccc(Cl)cc1)C(CC(F)(F)F)n1c2ccccc2c2ccccc21</smiles>

4o: $78.5 \mathrm{mg}, 98 \%$ yield, colorless oil, $12 \mathrm{~h}$, hexane: $\mathrm{EA}=10: 1, \mathrm{R}_{\mathrm{f}}=0.3$. 
${ }^{\mathbf{1}} \mathbf{H}$ NMR (400 MHz, Chloroform- $d$ ) $\delta 8.08(\mathrm{~d}, J=7.8 \mathrm{~Hz}, 2 \mathrm{H}), 7.73(\mathrm{~d}, J=8.7 \mathrm{~Hz}$, $2 \mathrm{H}), 7.67-7.24(\mathrm{~m}, 6 \mathrm{H}), 7.16(\mathrm{~d}, J=8.7 \mathrm{~Hz}, 2 \mathrm{H}), 6.22(\mathrm{dd}, J=8.3,4.1 \mathrm{~Hz}, 1 \mathrm{H}), 3.97$ $-3.37(\mathrm{~m}, 1 \mathrm{H}), 2.96-3.04(\mathrm{~m}, 1 \mathrm{H})$.

${ }^{19}$ F NMR (376 MHz, Chloroform- $d$ ) $\delta-64.16$.

${ }^{13}$ C NMR (100 MHz, Chloroform- $d$ ) $\delta$ 193.7, 140.6, 132.5, 131.5, 130.4, 129.4, 129.1, $128.5,126.5,126.2$ (q, $J=276.8 \mathrm{~Hz}), 123.9,122.1,120.8,120.4,53.7$ (q, $J=2.4 \mathrm{~Hz})$, $32.6(\mathrm{q}, J=29.0 \mathrm{~Hz})$.

IR (neat, ATR) 2971, 2960, 1682, 1590, 1483, 1223, 1088, 746, $723 \mathrm{~cm}^{-1}$.

HRMS (ESI): $m / z$ : calculated for $\mathrm{C}_{22} \mathrm{H}_{16} \mathrm{ClF}_{3} \mathrm{NO}^{+}[\mathrm{M}+\mathrm{H}]^{+}$402.0867, found: 402.0866 .<smiles>CC(=O)NC(CC(F)(F)F)C(=O)c1ccc(Cl)cc1</smiles>

4p: $37.3 \mathrm{mg}$, $64 \%$ yield, white solid, m.p. $176-180{ }^{\circ} \mathrm{C}, 12 \mathrm{~h}$, hexane: $\mathrm{EA}=3: 1, \mathrm{R}_{\mathrm{f}}=$ 0.4 .

${ }^{1} \mathbf{H}$ NMR (400 MHz, Chloroform- $d$ ) $\delta 7.95(\mathrm{~d}, J=8.7 \mathrm{~Hz}, 2 \mathrm{H}), 7.52(\mathrm{~d}, J=8.6 \mathrm{~Hz}, 2 \mathrm{H})$, $6.35(\mathrm{~d}, J=8.3 \mathrm{~Hz}, 1 \mathrm{H}), 5.98-5.63(\mathrm{~m}, 1 \mathrm{H}), 2.82-2.90(\mathrm{~m}, 1 \mathrm{H}), 2.64-2.44(\mathrm{~m}, 1 \mathrm{H})$, $2.07(\mathrm{~s}, 3 \mathrm{H})$.

${ }^{19}$ F NMR (376 MHz, Chloroform- $d$ ) $\delta-62.80$.

${ }^{13}$ C NMR (100 MHz, Chloroform- $d$ ) $\delta$ 195.1, 169.5, 141.0, 132.1, 130.1, 129.5, 125.5 $(\mathrm{q}, J=277.6 \mathrm{~Hz}), 48.2(\mathrm{q}, J=2.6 \mathrm{~Hz}), 36.0(\mathrm{q}, J=28.5 \mathrm{~Hz}), 23.1$.

IR (neat, ATR) 3300, 3075, 2972, 2901, 1685, 1658, 1588, 1572, 1533, 1490, 1127 , $1093,724,681 \mathrm{~cm}^{-1}$.

HRMS (ESI): $m / z$ : calculated for $\mathrm{C}_{12} \mathrm{H}_{12} \mathrm{ClF}_{3} \mathrm{NO}_{2}{ }^{+}[\mathrm{M}+\mathrm{H}]^{+}$294.0503, found: 294.0506.<smiles>COC(CC(F)(F)F)(C(=O)c1ccc(Cl)cc1)C(F)(F)F</smiles>

4q: $30.5 \mathrm{mg}, 54 \%$ yield, colorless oil, $12 \mathrm{~h}$, hexane: $\mathrm{EA}=20: 1, \mathrm{R}_{\mathrm{f}}=0.6$.

${ }^{1} \mathbf{H}$ NMR (400 MHz, Chloroform- $d$ ) $\delta 8.20(\mathrm{~d}, J=8.7 \mathrm{~Hz}, 2 \mathrm{H}), 7.45(\mathrm{~d}, J=8.7 \mathrm{~Hz}, 2 \mathrm{H})$, $3.27(\mathrm{~s}, 3 \mathrm{H}), 3.01-2.83(\mathrm{~m}, 1 \mathrm{H}), 2.82-2.64(\mathrm{~m}, 1 \mathrm{H}), 1.66(\mathrm{~s}, 3 \mathrm{H})$.

${ }^{19}$ F NMR (376 MHz, Chloroform- $d$ ) $\delta-59.90$.

${ }^{13}$ C NMR (100 MHz, Chloroform- $d$ ) $\delta$ 200.0, 139.8, 132.5, 131.4, 128.9, 125.7 (q, $J=$ $277.5 \mathrm{~Hz}), 82.7(\mathrm{q}, J=1.6 \mathrm{~Hz}), 52.4,38.4(\mathrm{q}, J=27.5 \mathrm{~Hz}), 22.5(\mathrm{q}, J=1.8 \mathrm{~Hz})$.

IR (neat, ATR) 2959, 2837, 1683, 1587, 1567, 1486, 1462, 1264, 1091,757, $748 \mathrm{~cm}^{-1}$. HRMS (ESI): $m / z$ : calculated for $\mathrm{C}_{12} \mathrm{H}_{13} \mathrm{ClF}_{3} \mathrm{O}_{2}{ }^{+}[\mathrm{M}+\mathrm{H}]^{+}$281.0551, found: 281.0554. 
$\overbrace{\mathrm{O}_{\mathrm{AC}}}^{\mathrm{Me}} \mathrm{CF}_{3}$

4r: $42.9 \mathrm{mg}, 70 \%$ yield, colorless oil, $12 \mathrm{~h}$, hexane: $\mathrm{EA}=20: 1, \mathrm{R}_{\mathrm{f}}=0.6$.

${ }^{1}$ H NMR (400 MHz, Chloroform- $d$ ) $\delta 7.99(\mathrm{~d}, J=8.7 \mathrm{~Hz}, 2 \mathrm{H}), 7.44(\mathrm{~d}, J=8.6 \mathrm{~Hz}, 2 \mathrm{H})$, 3.30-3.42 (m, 1H), 2.86-2.98 (m, 1H), $2.02(\mathrm{~s}, 3 \mathrm{H}), 1.87$ (s, 3H).

${ }^{19}$ F NMR (376 MHz, Chloroform- $d$ ) $\delta-60.11$.

${ }^{13}$ C NMR (101 MHz, Chloroform- $d$ ) $\delta$ 195.9, 170.4, 139.6, 131.4, 130.0, 129.0, 126.0 $(\mathrm{q}, J=277.3 \mathrm{~Hz}), 81.8(\mathrm{q}, J=2.1 \mathrm{~Hz}), 37.6(\mathrm{q}, J=27.6 \mathrm{~Hz}), 23.5(\mathrm{q}, J=2.1 \mathrm{~Hz}), 21.1$. IR (neat, ATR) 2988, 2901, 1731, 1683, 1587, 1569, 1488, 1455, 1264, 1092, $763,753 \mathrm{~cm}^{-1}$.

HRMS (ESI): $m / z$ : calculated for $\mathrm{C}_{13} \mathrm{H}_{13} \mathrm{ClF}_{3} \mathrm{O}_{3}{ }^{+}[\mathrm{M}+\mathrm{H}]^{+}$309.0500, found: 309.0502 .<smiles>O=C(c1ccc(Cl)cc1)C(CC(F)(F)F)c1ccccc1</smiles>

4s: $54.3 \mathrm{mg}, 79 \%$ yield, colorless oil, $12 \mathrm{~h}$, hexane: $\mathrm{EA}=10: 1, \mathrm{R}_{\mathrm{f}}=0.6$.

${ }^{1}$ H NMR (400 MHz, Chloroform- $d$ ) $\delta 7.92(\mathrm{~d}, J=8.6 \mathrm{~Hz}, 2 \mathrm{H}), 7.48(\mathrm{~d}, J=8.6 \mathrm{~Hz}$, $2 \mathrm{H}), 7.43-7.39(\mathrm{~m}, 1 \mathrm{H}), 7.38-7.31(\mathrm{~m}, 4 \mathrm{H}), 4.66(\mathrm{dd}, J=8.7,4.3 \mathrm{~Hz}, 1 \mathrm{H}), 3.46-$ $2.81(\mathrm{~m}, 1 \mathrm{H}), 2.88-2.48(\mathrm{~m}, 1 \mathrm{H})$.

${ }^{19}$ F NMR (376 MHz, Chloroform- $d$ ) $\delta-64.33$.

${ }^{13}$ C NMR (100 MHz, Chloroform- $d$ ) $\delta$ 191.2, 140.1, 135.4, 133.4, 130.1, 129.9, 129.5, $129.4,129.1,125.8(\mathrm{q}, J=277.7 \mathrm{~Hz}), 43.9(\mathrm{q}, J=2.5 \mathrm{~Hz}), 35.3(\mathrm{q}, J=28.8 \mathrm{~Hz})$.

IR (neat, ATR) 3075, 2969, 1681, 1590, 1571, 1488, 1439, 1256, 1093, 747, 690 $\mathrm{cm}^{-1}$.

HRMS (ESI): $m / z$ : calculated for $\mathrm{C}_{16} \mathrm{H}_{13} \mathrm{ClF}_{3} \mathrm{OS}^{+}[\mathrm{M}+\mathrm{H}]^{+}$345.0322, found: 345.0325 .<smiles>O=C(c1ccc(Cl)cc1)C(Cc1ccccc1)CC(F)(F)F</smiles>

4t: $13.6 \mathrm{mg}, 21 \%$ yield, colorless oil, $12 \mathrm{~h}$, hexane: $\mathrm{EA}=20: 1, \mathrm{R}_{\mathrm{f}}=0.7$.

${ }^{1}$ H NMR (400 MHz, Chloroform- $d$ ) $\delta 7.90$ - $7.73(\mathrm{~m}, 2 \mathrm{H}), 7.46-7.39(\mathrm{~m}, 2 \mathrm{H}), 7.29$ $7.19(\mathrm{~m}, 3 \mathrm{H}), 7.17-7.05(\mathrm{~m}, 2 \mathrm{H}), 3.99(\mathrm{~m}, 1 \mathrm{H}), 3.08(\mathrm{dd}, J=13.7,7.0 \mathrm{~Hz}, 1 \mathrm{H}), 2.95-$ $2.67(\mathrm{~m}, 2 \mathrm{H}), 2.39-2.16(\mathrm{~m}, 1 \mathrm{H})$.

${ }^{19}$ F NMR (376 MHz, Chloroform- $d$ ) $\delta$-64.60.

${ }^{13}$ C NMR (100 MHz, Chloroform- $d$ ) $\delta$ 199.9, 140.0, 137.2, 134.6, 129.7, 129.1, 129.0, 128.8, 127.1, $126.4(\mathrm{q}, J=277.1 \mathrm{~Hz}), 41.8(\mathrm{q}, J=1.7 \mathrm{~Hz}), 39.0,35.0(\mathrm{q}, J=28.6 \mathrm{~Hz})$. 
IR (neat, ATR) 2970, 2950, 1684, 1590, 1489, 1251, 1093, 754, $700 \mathrm{~cm}^{-1}$.

HRMS (ESI): $m / z$ : calculated for $\mathrm{C}_{17} \mathrm{H}_{15} \mathrm{ClF}_{3} \mathrm{O}^{+}[\mathrm{M}+\mathrm{H}]^{+}$327.0758, found: 327.0755 .<smiles>O=C(c1ccc(Cl)cc1)C(CC(F)(F)F)CC(F)(F)F</smiles>

4u: $23.3 \mathrm{mg}, 38 \%$ yield, colorless oil, $12 \mathrm{~h}$, hexane: $\mathrm{EA}=20: 1, \mathrm{R}_{\mathrm{f}}=0.7$.

${ }^{1}$ H NMR (400 MHz, Chloroform- $d$ ) $\delta 7.95(\mathrm{~d}, J=8.6 \mathrm{~Hz}, 2 \mathrm{H}), 7.50(\mathrm{~d}, J=8.6 \mathrm{~Hz}, 2 \mathrm{H})$, $3.76-3.81(\mathrm{~m}, 1 \mathrm{H}), 2.66-2.80(\mathrm{~m}, 1 \mathrm{H}), 2.26-2.33(\mathrm{~m}, 1 \mathrm{H}), 1.90-1.95(\mathrm{~m}, 1 \mathrm{H}), 1.42-1.47$ (m, 1H), $0.90(\mathrm{~s}, 9 \mathrm{H})$.

${ }^{19}$ F NMR (376 MHz, Chloroform- $d$ ) $\delta$-64.66.

${ }^{13}$ C NMR (100 MHz, Chloroform- $d$ ) $\delta$ 200.7, 140.0, 134.5, 129.8, 129.2, 126.3 (q, $J=$ $277.3 \mathrm{~Hz}$ ), 46.3, 37.8 (q, $J=28.0 \mathrm{~Hz}$ ), 35.9 (q, $J=2.0 \mathrm{~Hz}), 31.2,29.8$.

IR (neat, ATR) 2961, 2834, 1686, 1590, 1571, 1477, 1438, 1248, 1094, $757 \mathrm{~cm}^{-1}$.

HRMS (ESI): $m / z$ : calculated for $\mathrm{C}_{15} \mathrm{H}_{19} \mathrm{ClF}_{3} \mathrm{O}^{+}[\mathrm{M}+\mathrm{H}]^{+}$307.1071, found: 307.1069.<smiles>CC(C)OC(=O)C(C)(C)Oc1ccc(C(=O)c2ccc(C(CC(F)(F)F)C(=O)c3ccc(Cl)cc3)cc2)cc1</smiles>

4v: $97.4 \mathrm{mg}, 87 \%$ yield, colorless oil, $12 \mathrm{~h}$, hexane: $\mathrm{EA}=10: 1, \mathrm{R}_{\mathrm{f}}=0.6$.

${ }^{1}$ H NMR $(400 \mathrm{MHz}$, Chloroform- $d) \delta 7.92(\mathrm{~d}, J=8.6 \mathrm{~Hz}, 2 \mathrm{H}), 7.82-7.67(\mathrm{~m}, 4 \mathrm{H})$, $7.53-7.39(\mathrm{~m}, 4 \mathrm{H}), 6.86(\mathrm{~d}, J=8.8 \mathrm{~Hz}, 2 \mathrm{H}), 5.10(\mathrm{~m}, 1 \mathrm{H}), 4.14(\mathrm{q}, J=7.1 \mathrm{~Hz}, 1 \mathrm{H})$, $3.46-3.08(\mathrm{~m}, 1 \mathrm{H}), 2.75-2.47(\mathrm{~m}, 1 \mathrm{H}), 1.67(\mathrm{~s}, 6 \mathrm{H}), 1.21(\mathrm{~d}, J=6.3 \mathrm{~Hz}, 6 \mathrm{H})$.

${ }^{19}$ F NMR (376 MHz, Chloroform- $d$ ) $\delta-64.47$.

${ }^{13}$ C NMR (100 MHz, Chloroform- $d$ ) $\delta$ 195.1, 194.6, 173.1, 159.8, 140.9, 140.3, 137.9, 133.7, 132.0, 130.8, 130.2, 130.1, 129.2, 127.9, 126.2 (q, $J=275.0 \mathrm{~Hz}), 117.2,79.4$, $77.2,69.4,47.1(\mathrm{q}, J=2.4 \mathrm{~Hz}), 37.2(\mathrm{q}, J=28.5 \mathrm{~Hz}), 25.4,21.5$, missing one carbon.

IR (neat, ATR) 2986, 2970, 1729, 1687, 1652, 1598, 1572, 1504, 1489, 1252, 1098, $765,731 \mathrm{~cm}^{-1}$.

HRMS (ESI): $m / z$ : calculated for $\mathrm{C}_{30} \mathrm{H}_{29} \mathrm{ClF}_{3} \mathrm{O}_{5}{ }^{+}[\mathrm{M}+\mathrm{H}]^{+}$561.1648, found: 561.1650 .<smiles>O=C(c1ccccc1)C(CC(F)(F)F)c1ccccc1</smiles>

5a: $45.6 \mathrm{mg}, 82 \%$ yield, colorless oil, $12 \mathrm{~h}$, hexane: $\mathrm{EA}=20: 1, \mathrm{R}_{\mathrm{f}}=0.6$.

${ }^{1} \mathbf{H}$ NMR (400 MHz, Chloroform- $d$ ) $\delta 8.22$ - $7.88(\mathrm{~m}, 2 \mathrm{H}), 7.58-7.51(\mathrm{~m}, 1 \mathrm{H}), 7.47$ - 
$7.41(\mathrm{~m}, 2 \mathrm{H}), 7.34(\mathrm{~m}, 4 \mathrm{H}), 7.30-7.22(\mathrm{~m}, 1 \mathrm{H}), 4.95(\mathrm{dd}, J=7.6,5.5 \mathrm{~Hz}, 1 \mathrm{H}), 3.60-$ $3.05(\mathrm{~m}, 1 \mathrm{H}), 2.52-2.65(\mathrm{~m}, 1 \mathrm{H})$.

${ }^{19}$ F NMR (376 MHz, Chloroform- $d$ ) $\delta-64.59$.

${ }^{13}$ C NMR (100 MHz, Chloroform- $d$ ) $\delta 196.8,137.4,135.7,133.4,129.4,128.8,128.7$, 128.1, 127.9, 126.4 (q, $J=277.2 \mathrm{~Hz}), 47.2(\mathrm{q}, J=2.4 \mathrm{~Hz}), 37.4(\mathrm{q}, J=28.2 \mathrm{~Hz})$.

IR (neat, ATR) 2988,2901, 1692, 1614, 1532, 1493, 1471, 1349, 1261, 722, $699 \mathrm{~cm}^{-1}$. HRMS (ESI): $m / z$ : calculated for $\mathrm{C}_{16} \mathrm{H}_{14} \mathrm{~F}_{3} \mathrm{O}^{+}[\mathrm{M}+\mathrm{H}]^{+} 279.0991$, found: 279.0987 .<smiles>O=C(c1ccc(C(F)(F)F)cc1)C(CC(F)(F)F)c1ccccc1</smiles>

5b: $49.8 \mathrm{mg}, 72 \%$ yield, colorless oil, $12 \mathrm{~h}$, hexane: $\mathrm{EA}=20: 1, \mathrm{R}_{\mathrm{f}}=0.6$.

${ }^{1}$ H NMR (400 MHz, Chloroform- $d$ ) $\delta 8.07(\mathrm{~d}, J=8.2 \mathrm{~Hz}, 2 \mathrm{H}), 7.69(\mathrm{~d}, J=8.3 \mathrm{~Hz}, 2 \mathrm{H})$, $7.51-7.15(\mathrm{~m}, 5 \mathrm{H}), 4.91(\mathrm{dd}, J=7.5,5.6 \mathrm{~Hz}, 1 \mathrm{H}), 3.52-3.11(\mathrm{~m}, 1 \mathrm{H}), 2.52-2.65(\mathrm{~m}$, $1 \mathrm{H})$.

${ }^{19}$ F NMR (376 MHz, Chloroform- $d$ ) $\delta-63.28,-64.64$.

${ }^{13}$ C NMR (100 MHz, Chloroform- $d$ ) $\delta$ 195.8, 138.4, 136.6, 134.6 (q, $J=32.8 \mathrm{~Hz}$ ), 129.6, 129.1, 128.2, 128.0, $125.8(\mathrm{q}, J=265.0 \mathrm{~Hz}), 124.9$ (d, $J=9.4 \mathrm{~Hz}), 122.1,47.7$ (q, $J=2.5 \mathrm{~Hz}), 37.3(\mathrm{q}, J=28.4 \mathrm{~Hz})$.

IR (neat, ATR) 2988, 2950, 1690, 1595, 1582, 1511, 1494, 1290, 1094, 751, 698 $\mathrm{cm}^{-1}$.

HRMS (ESI): $m / z$ : calculated for $\mathrm{C}_{17} \mathrm{H}_{13} \mathrm{~F}_{6} \mathrm{O}^{+}[\mathrm{M}+\mathrm{H}]^{+}$347.0865, found: 347.0867 .<smiles>O=C(c1ccc(F)cc1)C(CC(F)(F)F)c1ccccc1</smiles>

5c: $51.5 \mathrm{mg}, 87 \%$ yield, colorless oil, $12 \mathrm{~h}$, hexane: $\mathrm{EA}=20: 1, \mathrm{R}_{\mathrm{f}}=0.6$.

${ }^{1} \mathbf{H}$ NMR (400 MHz, Chloroform- $d$ ) $\delta 8.20$ - $7.93(\mathrm{~m}, 2 \mathrm{H}), 7.54-7.21(\mathrm{~m}, 5 \mathrm{H}), 7.18-$ $6.91(\mathrm{~m}, 2 \mathrm{H}), 4.87(\mathrm{dd}, J=7.6,5.5 \mathrm{~Hz}, 1 \mathrm{H}), 3.60-3.13(\mathrm{~m}, 1 \mathrm{H}), 2.50-2.63(\mathrm{~m}, 1 \mathrm{H})$.

${ }^{19}$ F NMR (376 MHz, Chloroform- $d$ ) $\delta$-64.62, -104.34.

${ }^{13}$ C NMR (100 MHz, Chloroform- $d$ ) $\delta 195.1,165.8(\mathrm{~d}, J=255.8 \mathrm{~Hz}), 137.3,132.0(\mathrm{~d}$, $J=3.0 \mathrm{~Hz}), 131.5(\mathrm{~d}, J=9.4 \mathrm{~Hz}), 129.4,129.2,128.0,126.3(\mathrm{q}, J=277.1 \mathrm{~Hz}), 115.9(\mathrm{~d}$, $J=22.0 \mathrm{~Hz}), 47.2(\mathrm{q}, J=2.5 \mathrm{~Hz}), 37.3(\mathrm{q}, J=28.2 \mathrm{~Hz})$.

IR (neat, ATR) 2998, 2963, 1687, 1590, 1571, 1497, 1451, 1270, 1095, 763, 688 $\mathrm{cm}^{-1}$.

HRMS (ESI): $m / z$ : calculated for $\mathrm{C}_{16} \mathrm{H}_{13} \mathrm{~F}_{4} \mathrm{O}^{+}[\mathrm{M}+\mathrm{H}]^{+}$297.0897, found: 297.0894 . 
<smiles>Cc1ccc(C(=O)C(CC(F)(F)F)c2ccccc2)cc1</smiles>

5d: $50.2 \mathrm{mg}, 86 \%$ yield, colorless oil, $12 \mathrm{~h}$, hexane: $\mathrm{EA}=20: 1, \mathrm{R}_{\mathrm{f}}=0.5$.

${ }^{1}$ H NMR (400 MHz, Chloroform- $d$ ) $\delta 7.90(\mathrm{dd}, J=8.3,2.0 \mathrm{~Hz}, 2 \mathrm{H}), 7.29-7.34(\mathrm{~m}$, $4 \mathrm{H}), 7.30-7.14(\mathrm{~m}, 3 \mathrm{H}), 4.90-4.94(\mathrm{~m}, 1 \mathrm{H}), 3.69-3.16(\mathrm{~m}, 1 \mathrm{H}), 2.53-2.61(\mathrm{~m}, 1 \mathrm{H})$, $2.38(\mathrm{~s}, 3 \mathrm{H})$.

${ }^{19}$ F NMR (376 MHz, Chloroform- $d$ ) $\delta-64.58$.

${ }^{13}$ C NMR (100 MHz, Chloroform- $d$ ) $\delta$ 196.3, 144.4, 137.7, 133.1, 129.4, 129.3, 129.0, 128.0, 127.8, $126.5(\mathrm{q}, J=277.1 \mathrm{~Hz}), 47.0(\mathrm{q}, J=2.6 \mathrm{~Hz}), 37.3(\mathrm{q}, J=28.1 \mathrm{~Hz}), 21.6$. IR (neat, ATR) 2988, 2901, 1671, 1605, 1573, 1495, 1456, 1254, 1097, 761, 699 $\mathrm{cm}^{-1}$.

HRMS (ESI): $m / z$ : calculated for $\mathrm{C}_{17} \mathrm{H}_{15} \mathrm{~F}_{3} \mathrm{NaO}^{+}[\mathrm{M}+\mathrm{Na}]^{+}$315.0967, found: 315.0970 .<smiles>Cc1cccc(C(=O)C(CC(F)(F)F)c2ccccc2)c1</smiles>

5e: $37.4 \mathrm{mg}, 64 \%$ yield, colorless oil, $12 \mathrm{~h}$, hexane: $\mathrm{EA}=20: 1, \mathrm{R}_{\mathrm{f}}=0.6$.

${ }^{1} \mathbf{H}$ NMR (400 MHz, Chloroform- $d$ ) $\delta 7.91-7.70(\mathrm{~m}, 2 \mathrm{H}), 7.60-7.02(\mathrm{~m}, 7 \mathrm{H}), 4.93$ $(\mathrm{dd}, J=7.7,5.4 \mathrm{~Hz}, 1 \mathrm{H}), 3.55-3.04(\mathrm{~m}, 1 \mathrm{H}), 2.50-2.63(\mathrm{~m}, 1 \mathrm{H}), 2.39(\mathrm{~s}, 3 \mathrm{H})$.

${ }^{19}$ F NMR (376 MHz, Chloroform- $d$ ) $\delta$-64.62.

${ }^{13}$ C NMR (100 MHz, Chloroform- $d$ ) $\delta$ 196.9, 138.5, 137.5, 135.7, 134.2, 129.3, 129.3, $128.5,128.0,127.8,126.0,125.0,47.1(\mathrm{q}, J=2.4 \mathrm{~Hz}), 37.4(\mathrm{q}, J=28.2 \mathrm{~Hz}), 21.4$.

IR (neat, ATR) 2988, 2901, 1682, 1602, 1585, 1493, 1455, 1260, 1127, 770, 747 $\mathrm{cm}^{-1}$.

HRMS (ESI): $m / z$ : calculated for $\mathrm{C}_{17} \mathrm{H}_{15} \mathrm{~F}_{3} \mathrm{NaO}^{+}[\mathrm{M}+\mathrm{Na}]^{+}$315.0967, found: 315.0960 .<smiles>O=C(c1cccc([N+](=O)[O-])c1)C(CC(F)(F)F)c1ccccc1</smiles>

5f: $58.8 \mathrm{mg}, 91 \%$ yield, colorless oil, $12 \mathrm{~h}$, hexane: $\mathrm{EA}=5: 1, \mathrm{R}_{\mathrm{f}}=0.5$.

${ }^{1} \mathbf{H}$ NMR $(400 \mathrm{MHz}$, Chloroform- $d) \delta 8.81(\mathrm{t}, J=2.0 \mathrm{~Hz}, 1 \mathrm{H}), 8.50-8.36(\mathrm{~m}, 1 \mathrm{H})$, $8.29(\mathrm{~m}, 1 \mathrm{H}), 7.64(\mathrm{t}, J=8.0 \mathrm{~Hz}, 1 \mathrm{H}), 7.43-7.11(\mathrm{~m}, 5 \mathrm{H}), 4.91(\mathrm{dd}, J=7.7,5.4 \mathrm{~Hz}$, $1 \mathrm{H}), 3.62-3.02(\mathrm{~m}, 1 \mathrm{H}), 2.60(\mathrm{~m}, 1 \mathrm{H})$.

${ }^{19}$ F NMR (376 MHz, Chloroform- $d$ ) $\delta-64.57$. 
${ }^{13}$ C NMR (100 MHz, Chloroform- $d$ ) $\delta$ 194.5, 148.4, 136.8, 136.3, 134.3, 130.0, 129.7, $128.4,128.0,127.6,126.2(\mathrm{q}, J=277.2 \mathrm{~Hz}), 123.8,47.8(\mathrm{q}, J=2.5 \mathrm{~Hz}), 37.2(\mathrm{q}, J=$ $28.5 \mathrm{~Hz}$ ).

IR (neat, ATR) 3087, 2987, 1693, 1614, 1582, 1532, 1493, 1257, 1103, 712, 699 $\mathrm{cm}^{-1}$.

HRMS (ESI): $m / z$ : calculated for $\mathrm{C}_{16} \mathrm{H}_{13} \mathrm{~F}_{3} \mathrm{NO}_{3}{ }^{+}[\mathrm{M}+\mathrm{H}]^{+}$324.0842, found: 324.0845 .<smiles>N#Cc1ccccc1C(=O)C(CC(F)(F)F)c1ccccc1</smiles>

5g: $39.8 \mathrm{mg}, 66 \%$ yield, colorless oil, $12 \mathrm{~h}$, hexane: $\mathrm{EA}=10: 1, \mathrm{R}_{\mathrm{f}}=0.6$.

${ }^{1}$ H NMR (400 MHz, Chloroform- $\left.d\right) \delta 7.95-7.86(\mathrm{~m}, 1 \mathrm{H}), 7.82-7.76(\mathrm{~m}, 1 \mathrm{H})$, 7.58-7.66 (m, 2H), $7.49-7.19(\mathrm{~m}, 5 \mathrm{H}), 4.96-4.78(\mathrm{~m}, 1 \mathrm{H}), 3.59-3.03(\mathrm{~m}, 1 \mathrm{H})$, 2.53-2.66 (m, 1H).

${ }^{19}$ F NMR (376 MHz, Chloroform- $d$ ) $\delta-64.54$.

${ }^{13}$ C NMR (100 MHz, Chloroform- $d$ ) $\delta$ 195.3, 138.9, 135.9, 135.4, 132.5, 132.4, 129.6, $129.2,128.4,128.1,126.2(\mathrm{q}, J=276.9 \mathrm{~Hz}), 117.6,112.0,48.7(\mathrm{q}, J=2.5 \mathrm{~Hz}), 37.0(\mathrm{q}$, $J=28.5 \mathrm{~Hz})$.

IR (neat, ATR) 3067, 2956, 1693, 1592, 1572, 1492, 1455, 1257, 1105,762, $700 \mathrm{~cm}^{-1}$. HRMS (ESI): $m / z$ : calculated for $\mathrm{C}_{17} \mathrm{H}_{13} \mathrm{~F}_{3} \mathrm{NO}^{+}[\mathrm{M}+\mathrm{H}]^{+}$304.0944, found: 304.0947 .<smiles>O=C(c1ccc(F)c(F)c1)C(CC(F)(F)F)c1ccccc1</smiles>

5h: $52.7 \mathrm{mg}, 84 \%$ yield, colorless oil, $12 \mathrm{~h}$, hexane: $\mathrm{EA}=20: 1, \mathrm{R}_{\mathrm{f}}=0.7$.

${ }^{1}$ H NMR (400 MHz, Chloroform- $d$ ) $\delta$ 7.74-7.84 (m, 2H), $7.44-7.27(\mathrm{~m}, 5 \mathrm{H})$, 7.19-7.24 (m, 1H), $4.82(\mathrm{dd}, J=7.7,5.4 \mathrm{~Hz}, 1 \mathrm{H}), 3.46-3.10(\mathrm{~m}, 1 \mathrm{H}), 2.49-2.62$ (m, $1 \mathrm{H})$.

${ }^{19}$ F NMR (376 MHz, Chloroform- $d$ ) $\delta-64.65,-128.80(\mathrm{~d}, J=20.9 \mathrm{~Hz}),-135.51(\mathrm{~d}, J=$ $21.0 \mathrm{~Hz}$ ).

${ }^{13}$ C NMR (100 MHz, Chloroform- $d$ ) $\delta$ 194.2, 153.7 (dd, $\left.J=257.9,12.9 \mathrm{~Hz}\right), 150.4(\mathrm{dd}$, $J=251.2,13.0 \mathrm{~Hz}), 136.8,132.7(\mathrm{dd}, J=4.0 \mathrm{~Hz}), 129.6,128.2,127.9,126.2(\mathrm{q}, J=$ $277.3 \mathrm{~Hz}), 125.9(\mathrm{dd}, J=7.4,3.6 \mathrm{~Hz}), 118.2(\mathrm{dd}, J=18.3,2.0 \mathrm{~Hz}), 117.6(\mathrm{~d}, J=18.1$ $\mathrm{Hz}), 47.4(\mathrm{q}, J=2.8 \mathrm{~Hz}), 37.3(\mathrm{q}, J=28.5 \mathrm{~Hz})$.

IR (neat, ATR) 3067, 2957, 1689, 1611, 1516, 1496, 1455, 1288, 1104, 749, 700 $\mathrm{cm}^{-1}$.

HRMS (ESI): $m / z$ : calculated for $\mathrm{C}_{16} \mathrm{H}_{12} \mathrm{~F}_{5} \mathrm{O}^{+}[\mathrm{M}+\mathrm{H}]^{+}$315.0803, found:315.0800 
<smiles>O=C(c1ccc2c(c1)OCCO2)C(CC(F)(F)F)c1ccccc1</smiles>

5i: $59.7 \mathrm{mg}, 89 \%$ yield, colorless oil, $12 \mathrm{~h}$, hexane: $\mathrm{EA}=10: 1, \mathrm{R}_{\mathrm{f}}=0.4$.

${ }^{1}$ H NMR (400 MHz, Chloroform- $d$ ) $\delta$ 7.52-7.55 (m, 2H), 7.37 - 7.31 (m, 4H), 7.29 $7.22(\mathrm{~m}, 1 \mathrm{H}), 6.98-6.76(\mathrm{~m}, 1 \mathrm{H}), 4.84(\mathrm{dd}, J=7.7,5.5 \mathrm{~Hz}, 1 \mathrm{H}), 4.39-4.07$ (m, 4H), 3.26-3.34 (m, 1H), 2.50-2.58 (m, 1H).

${ }^{19}$ F NMR (376 MHz, Chloroform- $d$ ) $\delta-64.62$.

${ }^{13}$ C NMR (100 MHz, Chloroform- $d$ ) $\delta$ 195.1, 148.3, 143.4, 137.8, 129.4, 129.3, 128.0, $127.7,126.4(\mathrm{q}, J=277.3 \mathrm{~Hz}), 123.1,118.4,117.4,64.7,64.1,46.8(\mathrm{q}, J=2.4 \mathrm{~Hz}), 37.4$ $(\mathrm{q}, J=28.2 \mathrm{~Hz})$.

IR (neat, ATR) 3064, 2987, 2938, 2882, 1675, 1604, 1579, 1506, 1456, 1288, 1064, $744,699 \mathrm{~cm}^{-1}$.

HRMS (ESI): $m / z$ : calculated for $\mathrm{C}_{18} \mathrm{H}_{16} \mathrm{~F}_{3} \mathrm{O}_{3}{ }^{+}[\mathrm{M}+\mathrm{H}]^{+}$337.1046, found: 337.1043 .<smiles>O=C(c1ccc2ccccc2c1)C(CC(F)(F)F)c1ccccc1</smiles>

5j: $49.8 \mathrm{mg}, 76 \%$ yield, colorless oil, $12 \mathrm{~h}$, hexane: $\mathrm{EA}=20: 1, \mathrm{R}_{\mathrm{f}}=0.6$.

${ }^{1}$ H NMR (400 MHz, Chloroform- $d$ ) $\delta 8.53(\mathrm{~s}, 1 \mathrm{H}), 8.04(\mathrm{dd}, J=8.7,1.7 \mathrm{~Hz}, 1 \mathrm{H}), 7.95$ $(\mathrm{d}, J=8.0 \mathrm{~Hz}, 1 \mathrm{H}), 7.89-7.76(\mathrm{~m}, 2 \mathrm{H}), 7.67-7.50(\mathrm{~m}, 2 \mathrm{H}), 7.46-7.16(\mathrm{~m}, 5 \mathrm{H}), 5.11$ $(\mathrm{dd}, J=7.5,5.6 \mathrm{~Hz}, 1 \mathrm{H}), 3.69-2.96(\mathrm{~m}, 1 \mathrm{H}), 2.81-2.38(\mathrm{~m}, 1 \mathrm{H})$.

${ }^{19}$ F NMR (376 MHz, Chloroform- $d$ ) $\delta-64.49$.

${ }^{13}$ C NMR (100 MHz, Chloroform- $d$ ) $\delta$ 196.7, 137.6, 135.6, 133.0, 132.4, 130.7, 129.7, $129.4,128.8,128.6,128.1,127.9,127.7,126.9,125.1,124.4,47.3$ (q, $J=2.5 \mathrm{~Hz}), 37.4$ $(\mathrm{q}, J=28.2 \mathrm{~Hz})$.

IR (neat, ATR) 3063, 2961, 1674, 1627, 1598, 1494, 1468, 1260, 1107, 749, 700 $\mathrm{cm}^{-1}$.

HRMS (ESI): $m / z$ : calculated for $\mathrm{C}_{20} \mathrm{H}_{16} \mathrm{~F}_{3} \mathrm{O}^{+}[\mathrm{M}+\mathrm{H}]^{+}$329.1148, found:329.0044<smiles>O=C(c1cccnc1)C(CC(F)(F)F)c1ccccc1</smiles>

5k: $44.1 \mathrm{mg}, 79 \%$ yield, colorless oil, $12 \mathrm{~h}$, hexane: $\mathrm{EA}=5: 1, \mathrm{R}_{\mathrm{f}}=0.5$.

${ }^{1}$ H NMR (400 MHz, Chloroform- $d$ ) $\delta 9.19(\mathrm{~d}, J=2.3 \mathrm{~Hz}, 1 \mathrm{H}), 8.71(\mathrm{dd}, J=4.8,1.7$ $\mathrm{Hz}, 1 \mathrm{H}), 8.21-8.24(\mathrm{~m}, 1 \mathrm{H}), 7.61-6.85(\mathrm{~m}, 6 \mathrm{H}), 4.87$ (dd, $J=7.7,5.4 \mathrm{~Hz}, 1 \mathrm{H}), 3.75$ $-3.06(\mathrm{~m}, 1 \mathrm{H}), 2.81-2.34(\mathrm{~m}, 1 \mathrm{H})$.

${ }^{19}$ F NMR (376 MHz, Chloroform- $d$ ) $\delta-64.58$. 
${ }^{13}$ C NMR (100 MHz, Chloroform- $d$ ) $\delta$ 195.6, 153.6, 150.1, 136.5, 136.2, 131.0, 129.6, $128.2,128.1,126.3(\mathrm{q}, J=277.3 \mathrm{~Hz}), 123.7,47.8(\mathrm{q}, J=2.5 \mathrm{~Hz}), 37.0(\mathrm{q}, J=28.3$ $\mathrm{Hz})$.

IR (neat, ATR) 3010, 2959, 1689, 1585, 1570, 1493, 1455, 1259, 1137, $699 \mathrm{~cm}^{-1}$.

HRMS (ESI): $m / z$ : calculated for $\mathrm{C}_{15} \mathrm{H}_{13} \mathrm{~F}_{3} \mathrm{NO}^{+}[\mathrm{M}+\mathrm{H}]^{+}$280.0944, found: 280.0943.<smiles>O=C(c1ccn([12F])c1)C(CC(F)(F)F)c1ccccc1</smiles>

5l: $70.5 \mathrm{mg}, 84 \%$ yield, colorless oil, $12 \mathrm{~h}$, hexane: $\mathrm{EA}=10: 1, \mathrm{R}_{\mathrm{f}}=0.6$.

${ }^{1}$ H NMR (400 MHz, Chloroform- $d$ ) $\delta 7.73(\mathrm{dd}, J=5.1,3.1 \mathrm{~Hz}, 3 \mathrm{H}), 7.51-7.20(\mathrm{~m}$, $7 \mathrm{H}), 7.05(\mathrm{dd}, J=3.3,2.2 \mathrm{~Hz}, 1 \mathrm{H}), 6.65(\mathrm{dd}, J=3.3,1.6 \mathrm{~Hz}, 1 \mathrm{H}), 4.60-4.29(\mathrm{~m}, 1 \mathrm{H})$, $3.53-3.16(\mathrm{~m}, 1 \mathrm{H}), 2.57-2.46(\mathrm{~m}, 1 \mathrm{H}), 2.43(\mathrm{~s}, 3 \mathrm{H})$.

${ }^{19}$ F NMR (376 MHz, Chloroform- $d$ ) $\delta-64.56$.

${ }^{13}$ C NMR (100 MHz, Chloroform- $d$ ) $\delta$ 191.6, 146.0, 137.7, 134.8, 130.3, 129.3, 128.0, $127.9,127.3,127.2,126.3(\mathrm{q}, J=277.1 \mathrm{~Hz}), 125.1,121.5,112.8,49.0(\mathrm{q}, J=2.4 \mathrm{~Hz})$, $36.8(\mathrm{q}, J=28.3 \mathrm{~Hz}), 21.7$.

IR (neat, ATR) 3151, 2988, 1660, 1595, 1535, 1478, 1455, 1375, 1069, $682 \mathrm{~cm}^{-1}$.

HRMS (ESI): $m / z$ : calculated for $\mathrm{C}_{21} \mathrm{H}_{19} \mathrm{~F}_{3} \mathrm{NO}_{3} \mathrm{~S}^{+}[\mathrm{M}+\mathrm{H}]^{+}$422.1032, found: 422.1030 .<smiles>O=C(c1ccoc1)C(CC(F)(F)F)c1ccccc1</smiles>

5m: $28.4 \mathrm{mg}, 53 \%$ yield, colorless oil, $12 \mathrm{~h}$, hexane: $\mathrm{EA}=20: 1, \mathrm{R}_{\mathrm{f}}=0.3$.

${ }^{1}$ H NMR (400 MHz, Chloroform- $d$ ) $\delta 8.13-7.82(\mathrm{~m}, 1 \mathrm{H}), 7.58-7.05(\mathrm{~m}, 6 \mathrm{H}), 6.75$ $(\mathrm{dd}, J=1.9,0.7 \mathrm{~Hz}, 1 \mathrm{H}), 4.44(\mathrm{dd}, J=7.1,6.1 \mathrm{~Hz}, 1 \mathrm{H}), 3.47-3.12(\mathrm{~m}, 1 \mathrm{H}), 2.66-2.34$ $(\mathrm{m}, 1 \mathrm{H})$.

${ }^{19}$ F NMR (376 MHz, Chloroform- $d$ ) $\delta-64.64$.

${ }^{13}$ C NMR (100 MHz, Chloroform- $d$ ) $\delta$ 191.4, 147.9, 144.2, 137.5, 129.4, 128.0, 127.9, $126.4,126.3$ (q, $J=277.2 \mathrm{~Hz}), 109.1,49.9$ (q, $J=2.5 \mathrm{~Hz}), 36.7$ (q, $J=28.4 \mathrm{~Hz})$.

IR (neat, ATR) 3138, 2921, 1664, 1562, 1519, 1147, 1020, 872, $695 \mathrm{~cm}^{-1}$.

HRMS (ESI): $m / z$ : calculated for $\mathrm{C}_{14} \mathrm{H}_{12} \mathrm{~F}_{3} \mathrm{O}_{2}{ }^{+}[\mathrm{M}+\mathrm{H}]^{+}$269.0784, found: 269.0781 .<smiles>O=C(c1ccco1)C(CC(F)(F)F)c1ccccc1</smiles>

5n: $39.1 \mathrm{mg}, 73 \%$ yield, colorless oil, $12 \mathrm{~h}$, hexane: $\mathrm{EA}=20: 1, \mathrm{R}_{\mathrm{f}}=0.3$. 
${ }^{1}$ H NMR (400 MHz, Chloroform- $d$ ) $\delta 7.68$ - $7.53(\mathrm{~m}, 1 \mathrm{H}), 7.41$ - 7.31 (m, 4H), 7.30 $7.20(\mathrm{~m}, 2 \mathrm{H}), 6.52(\mathrm{dd}, J=3.6,1.7 \mathrm{~Hz}, 1 \mathrm{H}), 4.75(\mathrm{dd}, J=8.0,5.4 \mathrm{~Hz}, 1 \mathrm{H}), 3.23-3.27$ (m, $1 \mathrm{H}), 2.49-2.62(\mathrm{~m}, 1 \mathrm{H})$.

${ }^{19}$ F NMR (376 MHz, Chloroform- $d$ ) $\delta-64.83$.

${ }^{13}$ C NMR (100 MHz, Chloroform- $d$ ) $\delta$ 185.7, 151.5, 146.9, 137.1, 129.2, 128.1, 127.9, $126.3(\mathrm{q}, J=277.0 \mathrm{~Hz}), 118.7,112.6,47.2(\mathrm{q}, J=2.3 \mathrm{~Hz}), 36.4(\mathrm{q}, J=28.5 \mathrm{~Hz})$.

IR (neat, ATR) 3127, 2969, 1656, 1600, 1564, 1497, 1466, 1135, 1070, 740, 697 $\mathrm{cm}^{-1}$.

HRMS (ESI): $m / z$ : calculated for $\mathrm{C}_{14} \mathrm{H}_{12} \mathrm{~F}_{3} \mathrm{O}_{2}{ }^{+}[\mathrm{M}+\mathrm{H}]^{+}$269.0784, found: 269.0782 .<smiles>O=C(c1ccsc1)C(CC(F)(F)F)c1ccccc1</smiles>

5o: $38.0 \mathrm{mg}, 67 \%$ yield, colorless oil, $12 \mathrm{~h}$, hexane: $\mathrm{EA}=20: 1, \mathrm{R}_{\mathrm{f}}=0.4$.

${ }^{1} \mathbf{H}$ NMR (400 MHz, Chloroform- $d$ ) $\delta 8.09(\mathrm{dd}, J=2.9,1.2 \mathrm{~Hz}, 1 \mathrm{H}), 7.55(\mathrm{dd}, J=5.1$, $1.2 \mathrm{~Hz}, 1 \mathrm{H}), 7.39-7.32(\mathrm{~m}, 4 \mathrm{H}), 7.27-7.30(\mathrm{~m}, 2 \mathrm{H}), 4.70(\mathrm{dd}, J=7.3,5.8 \mathrm{~Hz}, 1 \mathrm{H}), 3.85$ $-3.03(\mathrm{~m}, 1 \mathrm{H}), 2.48-2.61(\mathrm{~m}, 1 \mathrm{H})$.

${ }^{19}$ F NMR (376 MHz, Chloroform- $d$ ) $\delta-64.58$.

${ }^{13}$ C NMR (100 MHz, Chloroform- $d$ ) $\delta$ 190.8, 140.7, 137.6, 133.3, 129.4, 128.0, 127.9, $127.4,126.5,126.4(\mathrm{q}, J=277.1 \mathrm{~Hz}), 49.0(\mathrm{q}, J=2.4 \mathrm{~Hz}), 37.1(\mathrm{q}, J=28.2 \mathrm{~Hz})$.

IR (neat, ATR) 3108, 2958, 1674, 1601, 1585, 1509, 1495, 1257, 1136, 741, 698 $\mathrm{cm}^{-1}$.

HRMS (ESI): $m / z$ : calculated for $\mathrm{C}_{14} \mathrm{H}_{12} \mathrm{~F}_{3} \mathrm{OS}^{+}[\mathrm{M}+\mathrm{H}]^{+}$285.0555, found: 285.0556 .<smiles>O=C(c1cccs1)C(CC(F)(F)F)c1ccccc1</smiles>

5p: $26.1 \mathrm{mg}, 46 \%$ yield, colorless oil, $12 \mathrm{~h}$, hexane: $\mathrm{EA}=20: 1, \mathrm{R}_{\mathrm{f}}=0.3$.

${ }^{1}$ H NMR (400 MHz, Chloroform- $d$ ) $\delta 7.77(\mathrm{~d}, J=3.2 \mathrm{~Hz}, 1 \mathrm{H}), 7.64(\mathrm{~d}, J=4.9 \mathrm{~Hz}, 1 \mathrm{H})$, $7.47-7.23(\mathrm{~m}, 5 \mathrm{H}), 7.14-7.01(\mathrm{~m}, 1 \mathrm{H}), 4.73(\mathrm{dd}, J=7.5,5.7 \mathrm{~Hz}, 1 \mathrm{H}), 3.46-3.09(\mathrm{~m}$, $1 \mathrm{H}), 2.50-2.62(\mathrm{~m}, 1 \mathrm{H})$.

${ }^{19}$ F NMR (376 MHz, Chloroform- $d$ ) $\delta-64.68$.

${ }^{13}$ C NMR (100 MHz, Chloroform- $d$ ) $\delta$ 189.6, 142.7, 137.5, 134.5, 132.9, 129.3, 128.3, 128.0, 128.0, $126.3(\mathrm{q}, J=277.2 \mathrm{~Hz}), 48.6(\mathrm{q}, J=2.3 \mathrm{~Hz}), 37.1(\mathrm{q}, J=28.3 \mathrm{~Hz})$.

IR (neat, ATR) 3091, 2958, 1601, 1585, 1517, 1492, 1471, 1259, 1136, 722, 698 $\mathrm{cm}^{-1}$.

HRMS (ESI): $m / z$ : calculated for $\mathrm{C}_{14} \mathrm{H}_{12} \mathrm{~F}_{3} \mathrm{OS}^{+}[\mathrm{M}+\mathrm{H}]^{+}$285.0555, found: 285.0558 . 
<smiles>O=C(c1cn([123I])cn1)C(CC(F)(F)F)c1ccccc1</smiles>

5q: $59.1 \mathrm{mg}, 70 \%$ yield, colorless oil, $12 \mathrm{~h}$, hexane: $\mathrm{EA}=10: 1, \mathrm{R}_{\mathrm{f}}=0.6$.

${ }^{1}$ H NMR (400 MHz, Chloroform- $d$ ) $\delta 7.95(\mathrm{~d}, J=1.3 \mathrm{~Hz}, 1 \mathrm{H}), 7.89(\mathrm{~d}, J=1.4 \mathrm{~Hz}, 1 \mathrm{H})$, $7.85(\mathrm{~d}, J=8.4 \mathrm{~Hz}, 2 \mathrm{H}), 7.38(\mathrm{dd}, J=9.5,7.8 \mathrm{~Hz}, 4 \mathrm{H}), 7.33-7.18(\mathrm{~m}, 3 \mathrm{H}), 5.16(\mathrm{dd}, J$ $=8.6,5.0 \mathrm{~Hz}, 1 \mathrm{H}), 3.20-3.34(\mathrm{~m}, 1 \mathrm{H}), 2.57-2.49(\mathrm{~m}, 1 \mathrm{H}), 2.47(\mathrm{~s}, 3 \mathrm{H})$.

${ }^{19}$ F NMR (376 MHz, Chloroform- $d$ ) $\delta-64.88$.

${ }^{13}$ C NMR (100 MHz, Chloroform- $d$ ) $\delta$ 192.4, 147.3, 141.8, 136.8, 136.0, 133.8, 130.7, $129.0,128.5,127.8,127.7,126.3(\mathrm{q}, J=277.0 \mathrm{~Hz}), 121.8,46.7(\mathrm{q}, J=2.8 \mathrm{~Hz}), 36.2(\mathrm{q}$, $J=28.4 \mathrm{~Hz}), 21.8$.

IR (neat, ATR) 3130, 1678, 1594, 1525, 1488, 1455, 1377, 1130, 741, $684 \mathrm{~cm}^{-1}$.

HRMS (ESI): $m / z$ : calculated for $\mathrm{C}_{20} \mathrm{H}_{18} \mathrm{~F}_{3} \mathrm{~N}_{2} \mathrm{O}_{3} \mathrm{~S}^{+}[\mathrm{M}+\mathrm{H}]^{+}$423.0985, found: 423.0984 .<smiles>O=C(c1cnc2ccccc2c1)C(CC(F)(F)F)c1ccccc1</smiles>

5r: $45.4 \mathrm{mg}, 69 \%$ yield, colorless oil, $12 \mathrm{~h}$, hexane: $\mathrm{EA}=10: 1, \mathrm{R}_{\mathrm{f}}=0.3$.

${ }^{1} \mathbf{H}$ NMR (400 MHz, Chloroform- $d$ ) $\delta 9.44(\mathrm{~d}, J=2.2 \mathrm{~Hz}, 1 \mathrm{H}), 8.74(\mathrm{~d}, J=2.0 \mathrm{~Hz}, 1 \mathrm{H})$, $8.12(\mathrm{~d}, J=8.5 \mathrm{~Hz}, 1 \mathrm{H}), 7.92(\mathrm{~d}, J=8.2 \mathrm{~Hz}, 1 \mathrm{H}), 7.81-7.84(\mathrm{~m}, 1 \mathrm{H}), 7.60-7.64(\mathrm{~m}, 1 \mathrm{H})$, $7.43-7.32(\mathrm{~m}, 4 \mathrm{H}), 7.25-7.29(\mathrm{~m}, 1 \mathrm{H}), 5.02(\mathrm{dd}, J=7.5,5.6 \mathrm{~Hz}, 1 \mathrm{H}), 3.73-3.22(\mathrm{~m}$, $1 \mathrm{H}), 2.57-2.70(\mathrm{~m}, 1 \mathrm{H})$.

${ }^{19}$ F NMR (376 MHz, Chloroform- $d$ ) $\delta-64.48$.

${ }^{13}$ C NMR (100 MHz, Chloroform- $d$ ) $\delta$ 195.5, 149.7, 149.3, 138.0, 136.7, 132.3, 129.6, $129.4,129.4,128.2,128.1,128.0,127.7,126.7,126.3(\mathrm{q}, J=277.1 \mathrm{~Hz}), 47.9$ (q, $J=2.5$ $\mathrm{Hz}), 37.1$ (q, $J=28.4 \mathrm{~Hz}$ ).

IR (neat, ATR) 3042, 2924, 1679, 1616, 1586, 1495, 1456, 1252, 1132, 747, 701 $\mathrm{cm}^{-1}$.

HRMS (ESI): $m / z$ : calculated for $\mathrm{C}_{19} \mathrm{H}_{15} \mathrm{~F}_{3} \mathrm{NO}^{+}[\mathrm{M}+\mathrm{H}]^{+}$330.1100, found: 330.1103 .<smiles>O=C(c1ccc2ncccc2c1)C(CC(F)(F)F)c1ccccc1</smiles>

5s: $52.6 \mathrm{mg}, 80 \%$ yield, colorless oil, $12 \mathrm{~h}$, hexane: $\mathrm{EA}=10: 1, \mathrm{R}_{\mathrm{f}}=0.4$.

${ }^{1}$ H NMR (400 MHz, Chloroform- $d$ ) $\delta 9.00(\mathrm{~s}, 1 \mathrm{H}), 8.49(\mathrm{~s}, 1 \mathrm{H}), 8.26(\mathrm{~d}, J=8.1 \mathrm{~Hz}$, $2 \mathrm{H}), 8.12(\mathrm{~d}, J=8.8 \mathrm{~Hz}, 1 \mathrm{H}), 7.54-7.09(\mathrm{~m}, 6 \mathrm{H}), 5.44-4.83(\mathrm{~m}, 1 \mathrm{H}), 3.63-3.30(\mathrm{~m}$, $1 \mathrm{H}), 2.59-2.66(\mathrm{~m}, 1 \mathrm{H})$. 
${ }^{19}$ F NMR (376 MHz, Chloroform- $d$ ) $\delta-64.54$.

${ }^{13}$ C NMR (100 MHz, Chloroform- $d$ ) $\delta$ 196.1, 152.9, 150.1, 137.6, 137.2, 133.5, 130.5, 130.3, 129.5, 128.0, 127.4, $126.4(\mathrm{q}, J=277.2 \mathrm{~Hz}), 122.0,47.5(\mathrm{q}, J=2.6 \mathrm{~Hz}), 37.4$ (q, $J=28.3 \mathrm{~Hz})$.

IR (neat, ATR) 3004, 1684, 1616, 1503, 1282, 1132, 753, $704 \mathrm{~cm}^{-1}$.

HRMS (ESI): $m / z$ : calculated for $\mathrm{C}_{19} \mathrm{H}_{15} \mathrm{~F}_{3} \mathrm{NO}^{+}[\mathrm{M}+\mathrm{H}]^{+}$330.1100, found: 330.1101 .<smiles>O=C(c1ccc2ccccc2n1)C(CC(F)(F)F)c1ccccc1</smiles>

5t: $28.1 \mathrm{mg}, 43 \%$ yield, colorless oil, $12 \mathrm{~h}$, hexane: $\mathrm{EA}=10: 1, \mathrm{R}_{\mathrm{f}}=0.4$.

${ }^{1}$ H NMR $(400 \mathrm{MHz}$, Chloroform- $d$ ) $\delta 8.26(\mathrm{dd}, J=19.4,8.4 \mathrm{~Hz}, 2 \mathrm{H}), 8.12(\mathrm{~d}, J=8.5$ $\mathrm{Hz}, 1 \mathrm{H}), 7.89-7.78(\mathrm{~m}, 2 \mathrm{H}), 7.64-7.68(\mathrm{~m}, 1 \mathrm{H}), 7.52(\mathrm{dd}, J=5.2,3.3 \mathrm{~Hz}, 2 \mathrm{H}), 7.33-$ $7.25(\mathrm{~m}, 2 \mathrm{H}), 7.23-7.13(\mathrm{~m}, 1 \mathrm{H}), 6.11(\mathrm{dd}, J=8.5,5.3 \mathrm{~Hz}, 1 \mathrm{H}), 3.32-3.46(\mathrm{~m}, 1 \mathrm{H})$, $2.81-2.50(\mathrm{~m}, 1 \mathrm{H})$.

${ }^{19}$ F NMR (376 MHz, Chloroform- $d$ ) $\delta$-64.67.

${ }^{13}$ C NMR (100 MHz, Chloroform- $d$ ) $\delta$ 198.3, 151.3, 147.0, 137.5, 137.0, 130.8, 130.1, $129.7,128.9,128.8,128.8,127.6,127.4,126.6$ (q, $J=277.3 \mathrm{~Hz}), 118.9,44.3$ (q, $J=$ $2.5 \mathrm{~Hz}), 36.4(\mathrm{q}, J=28.4 \mathrm{~Hz})$.

IR (neat, ATR) 3100, 1690, 1591, 1570, 1493, 1455, 1121, 1062, 741, $696 \mathrm{~cm}^{-1}$.

HRMS (ESI): $m / z$ : calculated for $\mathrm{C}_{19} \mathrm{H}_{15} \mathrm{~F}_{3} \mathrm{NO}^{+}[\mathrm{M}+\mathrm{H}]^{+}$330.1100, found: 330.1104 .<smiles>O=C(c1cc2ccccc2o1)C(CC(F)(F)F)c1ccccc1</smiles>

5u: $50.7 \mathrm{mg}, 80 \%$ yield, colorless oil, $12 \mathrm{~h}$, hexane: $\mathrm{EA}=10: 1, \mathrm{R}_{\mathrm{f}}=0.4$.

${ }^{1} \mathbf{H}$ NMR (400 MHz, Chloroform- $d$ ) $\delta 7.69(\mathrm{dd}, J=7.9,0.6 \mathrm{~Hz}, 1 \mathrm{H}), 7.63-7.56(\mathrm{~m}$, 2H), 7.49-7.51 (m, 1H), $7.44-7.24(\mathrm{~m}, 6 \mathrm{H}), 4.90(\mathrm{dd}, J=8.0,5.4 \mathrm{~Hz}, 1 \mathrm{H}), 3.59-3.14$ (m, 1H), 2.54-2.68 (m, 1H).

${ }^{19}$ F NMR (376 MHz, Chloroform- $d$ ) $\delta-64.76$.

${ }^{13}$ C NMR (100 MHz, Chloroform- $d$ ) $\delta 187.6,155.7,151.3,136.7,129.3,128.6,128.2$, $128.1,126.9,126.3$ (q, $J=277.2 \mathrm{~Hz}), 124.0,123.4,114.4,112.5,47.6$ (q, $J=2.3 \mathrm{~Hz})$, $36.4(\mathrm{q}, J=28.8 \mathrm{~Hz})$.

IR (neat, ATR) 3065, 2963, 1682, 1610, 1556, 1260, 1105, 798, 751, 741, $698 \mathrm{~cm}^{-1}$. HRMS (ESI): $m / z$ : calculated for $\mathrm{C}_{18} \mathrm{H}_{14} \mathrm{~F}_{3} \mathrm{O}_{2}{ }^{+}[\mathrm{M}+\mathrm{H}]^{+}$319.0940, found: 319.0938 . 


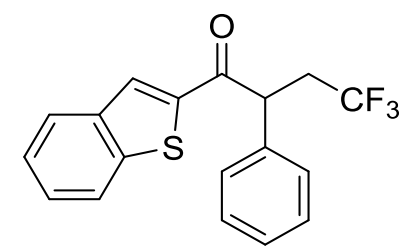

5v: $38.1 \mathrm{mg}, 57 \%$ yield, colorless oil, $12 \mathrm{~h}$, hexane: $\mathrm{EA}=10: 1, \mathrm{R}_{\mathrm{f}}=0.5$.

${ }^{1}$ H NMR (400 MHz, Chloroform- $d$ ) $\delta 8.09-7.99(\mathrm{~m}, 1 \mathrm{H}), 7.95-7.78(\mathrm{~m}, 2 \mathrm{H}), 7.50-$ $7.34(\mathrm{~m}, 6 \mathrm{H}), 7.32-7.24(\mathrm{~m}, 1 \mathrm{H}), 4.88(\mathrm{dd}, J=7.7,5.6 \mathrm{~Hz}, 1 \mathrm{H}), 3.50-3.13(\mathrm{~m}, 1 \mathrm{H})$, 2.56-2.68 (m, 1H).

${ }^{19}$ F NMR $(376 \mathrm{MHz}$, Chloroform- $d$ ) $\delta-64.61$.

${ }^{13}$ C NMR (100 MHz, Chloroform- $d$ ) $\delta$ 191.1, 142.7, 142.0, 138.9, 137.3, 130.2, 129.4, $128.1,128.0,127.7,126.3(\mathrm{q}, J=277.2 \mathrm{~Hz}), 126.2,125.1,122.9,48.5(\mathrm{q}, J=2.5 \mathrm{~Hz})$, $37.2(\mathrm{q}, J=28.4 \mathrm{~Hz})$.

IR (neat, ATR) 3067, 3002, 1680, 1589, 1571, 1490, 1456, 1284, 1133, 844, 804, 758, $702 \mathrm{~cm}^{-1}$.

HRMS (ESI): $m / z$ : calculated for $\mathrm{C}_{18} \mathrm{H}_{14} \mathrm{~F}_{3} \mathrm{OS}^{+}[\mathrm{M}+\mathrm{H}]^{+}$335.0712, found: 335.0715 . 


\section{The reaction of $1 \mathrm{a}$ with $2 \mathrm{a}$ at $1 \mathrm{mmol}$ Scale}<smiles>O=Cc1ccc(Cl)cc1</smiles>

1a<smiles></smiles>

2a

$3 a$

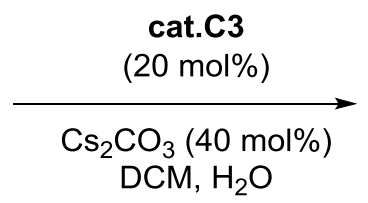<smiles>O=C(c1ccc(Cl)cc1)C(CC(F)(F)F)c1ccccc1</smiles>

$4 a$

To a flame-dried Schlenk reaction tube equipped with a magnetic stir bar, was added the pre-catalyst $\mathbf{C 3}(82.8 \mathrm{mg}, 0.4 \mathrm{mmol}), \mathrm{Cs}_{2} \mathrm{CO}_{3}(130 \mathrm{mg}, 0.8 \mathrm{mmol})$ and 3a (660 $\mathrm{mg}$, $2.0 \mathrm{mmol}$ ). The Schlenk tube was closed with a septum, evacuated and refilled with argon atmosphere. 1a (169.2 $\mathrm{mg}, 1.2 \mathrm{mmol})$ and 2a (104.1 mg, $1.0 \mathrm{mmol})$ in DCM (10 $\mathrm{mL})$ was added via a syringe. Water $(200 \mu \mathrm{L})$ was added subsequently. The mixture was then stirred at $25^{\circ} \mathrm{C}$ and monitored by TLC until 2 was consumed completely. The mixture was concentrated under reduced pressure and purified by flash column chromatography (hexane : EA, 20:1) to afford the trifluoromethylated product $4 \mathbf{a} 240$ mg as a white solid in $77 \%$ yield.

\section{Other scope of aldehydes}

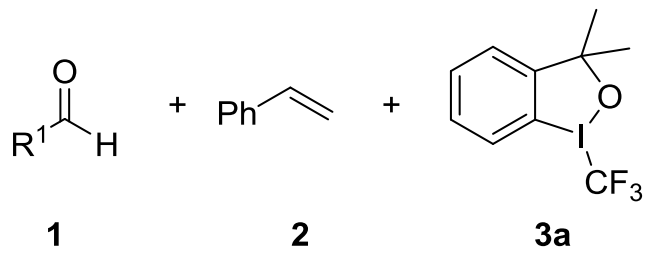

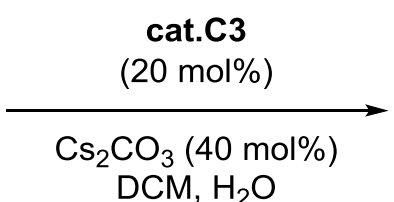<smiles>[R]C(=O)C(CC(F)(F)F)c1ccccc1</smiles>

5

all cases $<5 \%$ yield<smiles>CC(C)CC=O</smiles>

$(\mathrm{HCHO})_{n}$<smiles>O=CCCc1ccccc1</smiles><smiles>O=C/C=C/c1ccccc1</smiles><smiles>CC(=O)C=O</smiles><smiles>O=CC#Cc1ccccc1</smiles><smiles>CCOC(=O)C=O</smiles>

\section{References}

1. W. Liu, L.Li, Z. Chen, C.-J. Li, Org. Biomol. Chem., 2015, 13, 6170-6174. 


\section{NMR spectra}

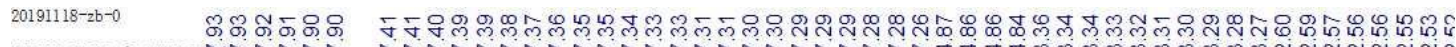

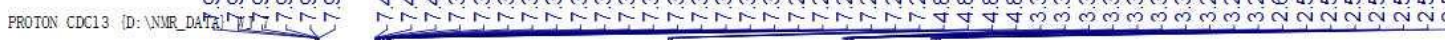

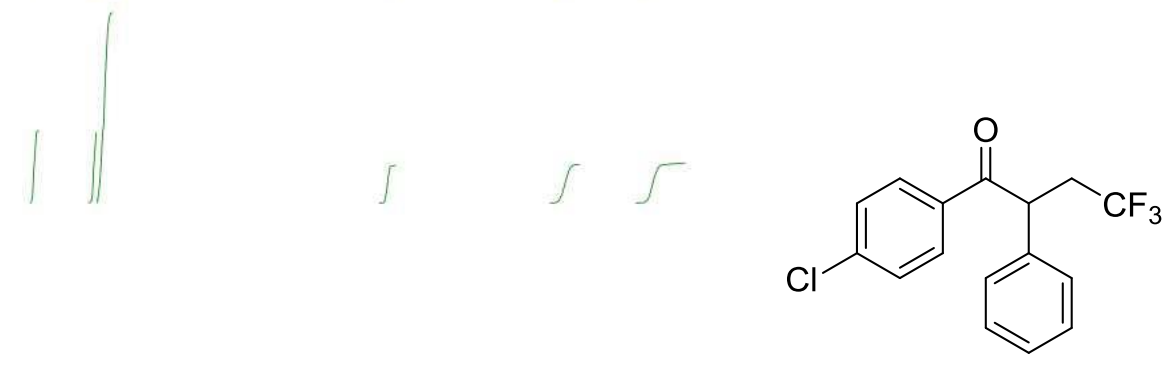

$4 a$

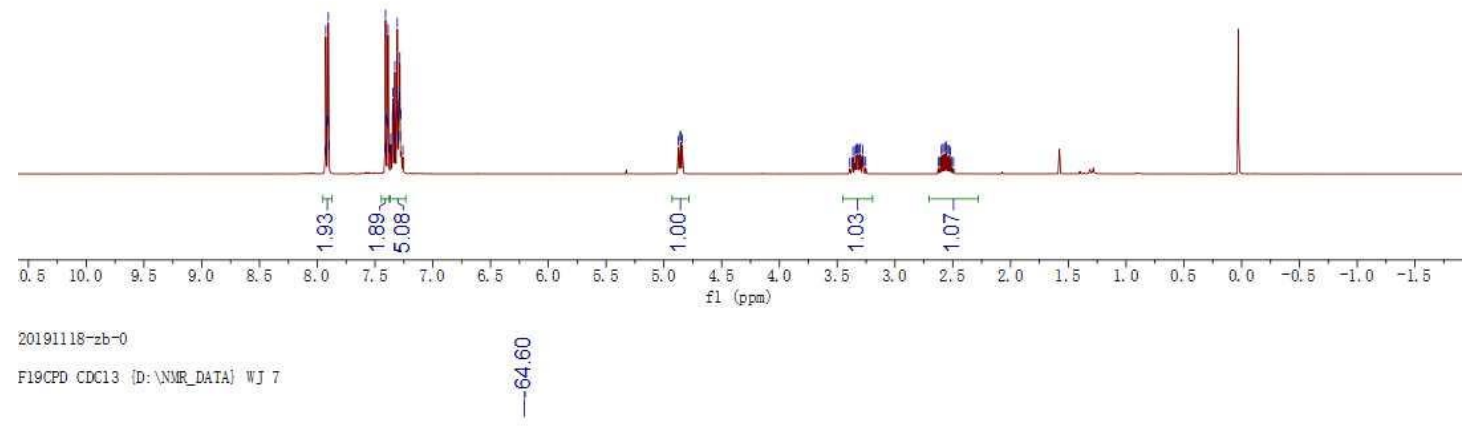<smiles>O=C(c1ccc(Cl)cc1)C(CC(F)(F)F)c1ccccc1</smiles>

4a

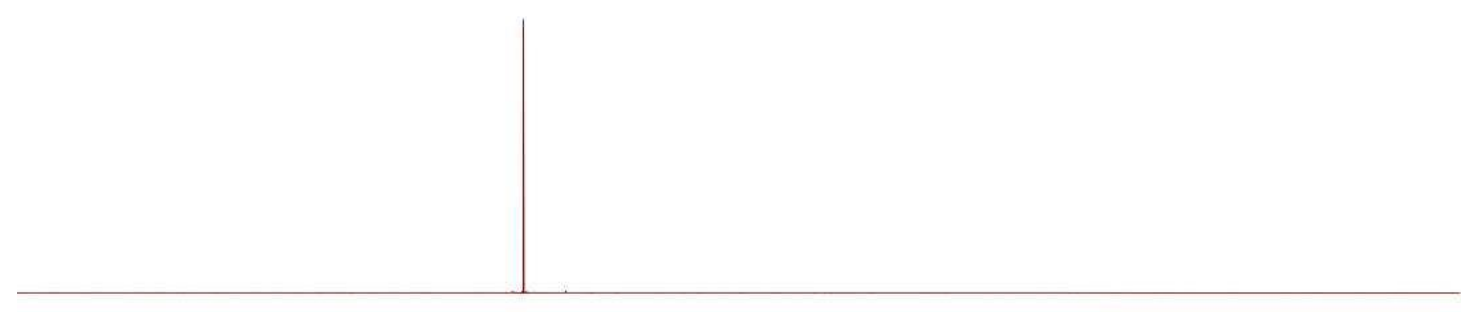

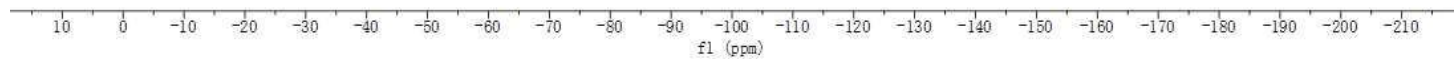


20191118-zb-0 กิ

C13CPD CDC13 [Dङू

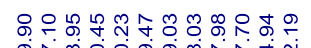

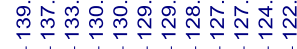<smiles>C=CC(C)CCCCCCCCCC(C)C</smiles>

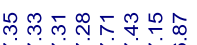

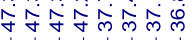

म

$4 a$

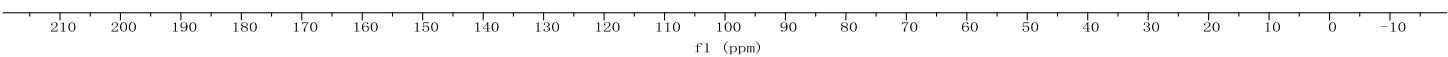



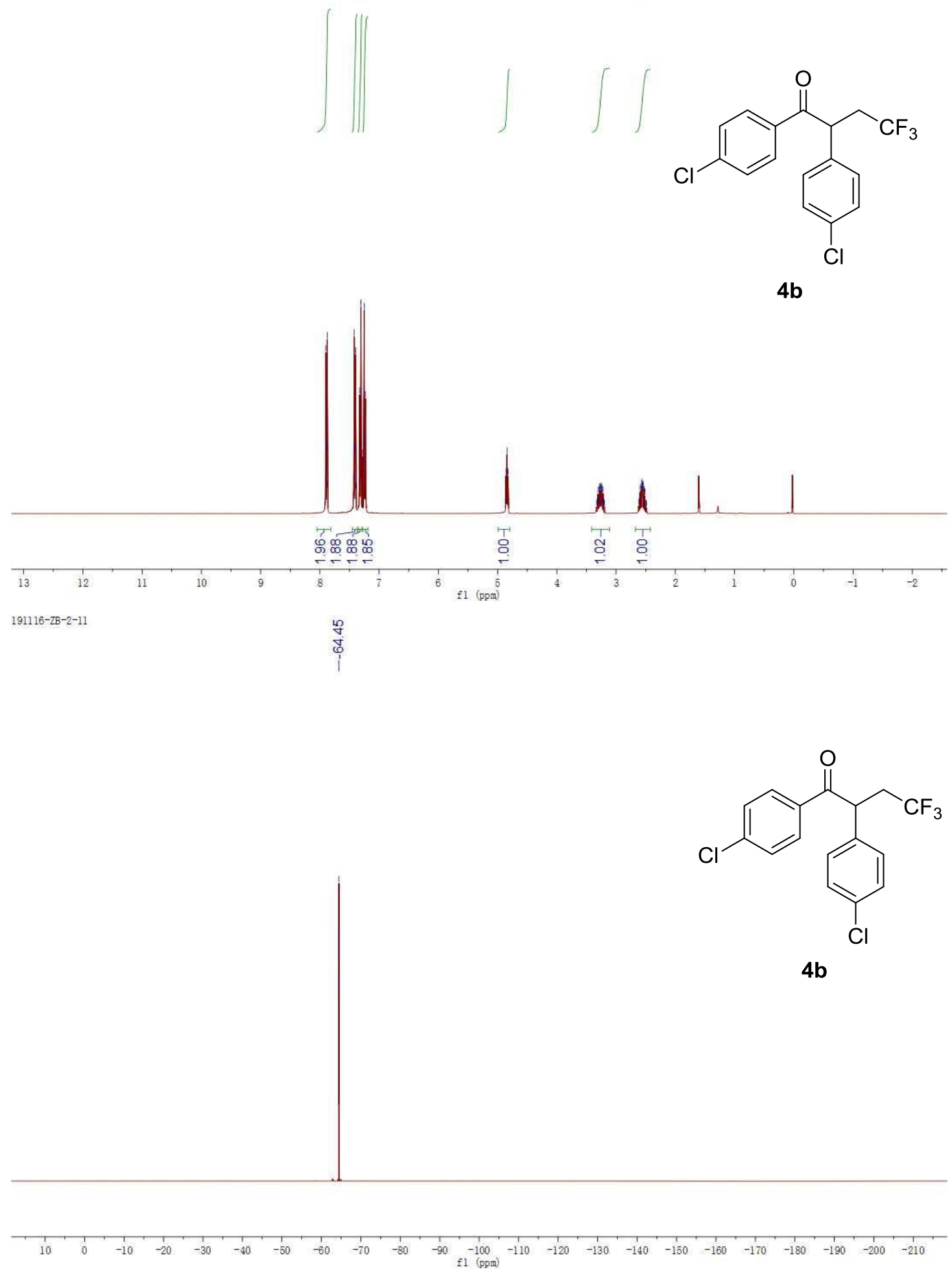


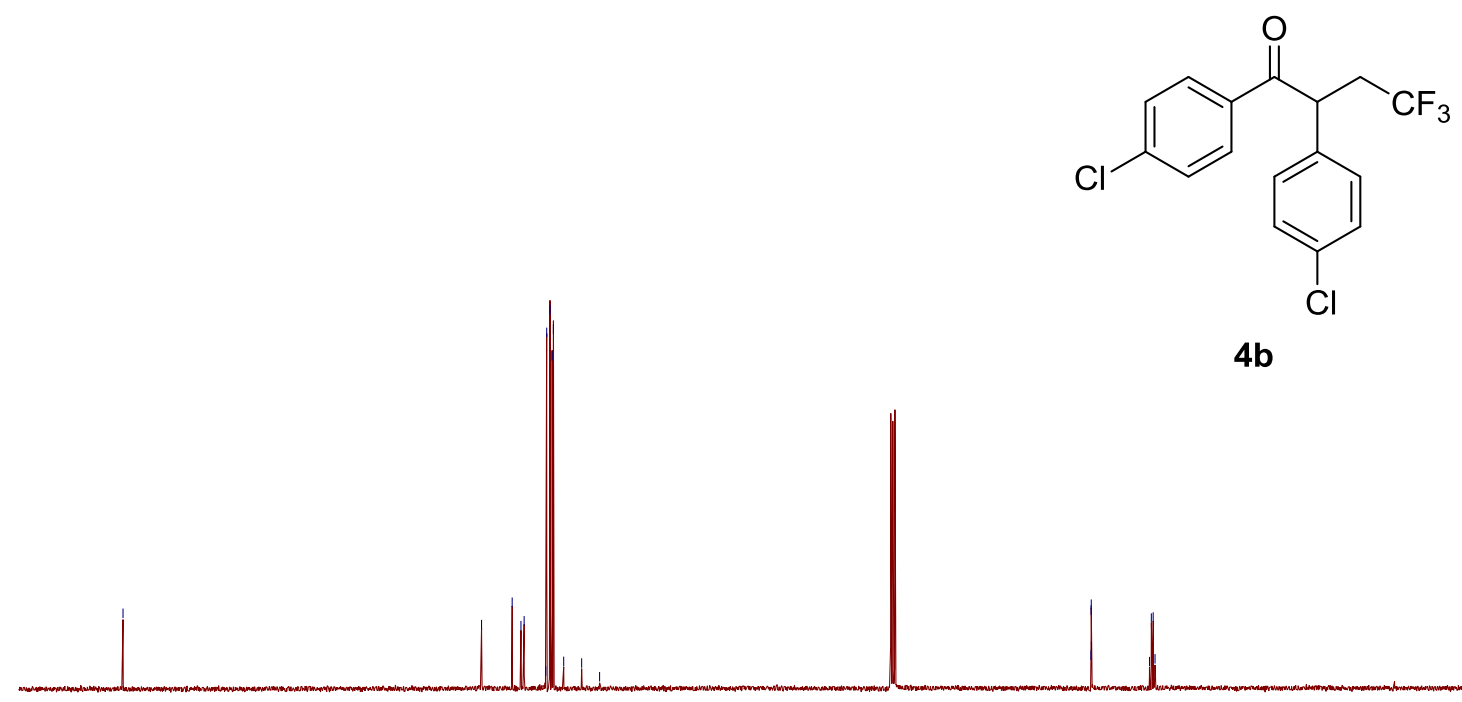

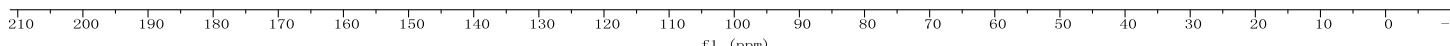




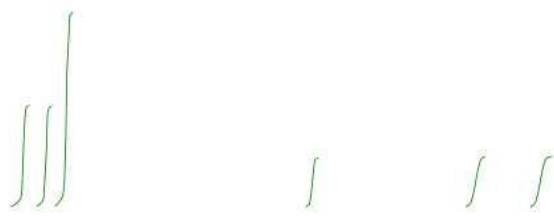<smiles>N#Cc1ccc(C(CC(F)(F)F)C(=O)c2ccc(Cl)cc2)cc1</smiles>

4c

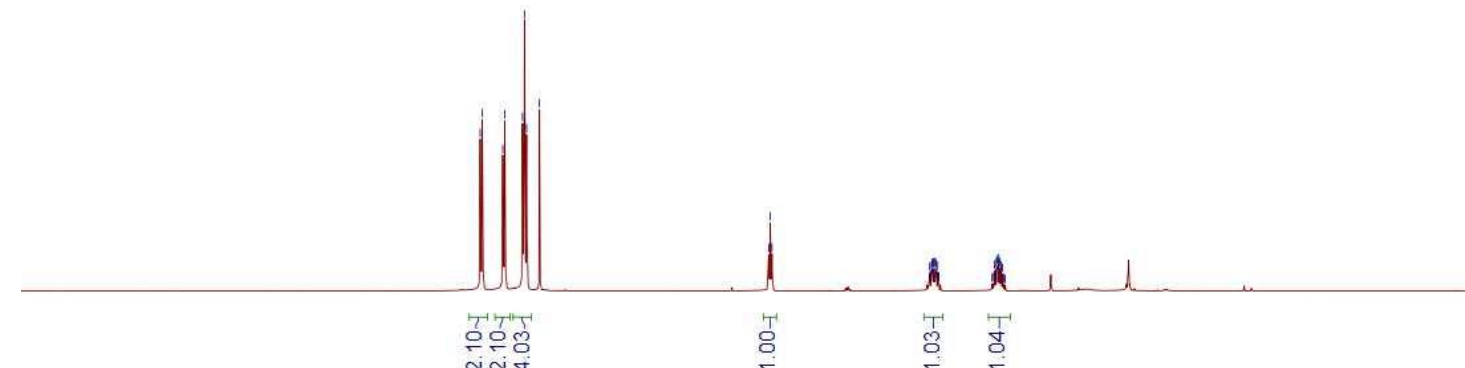

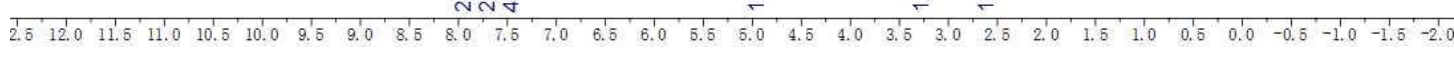
191122-zb-2-17. 11. fid

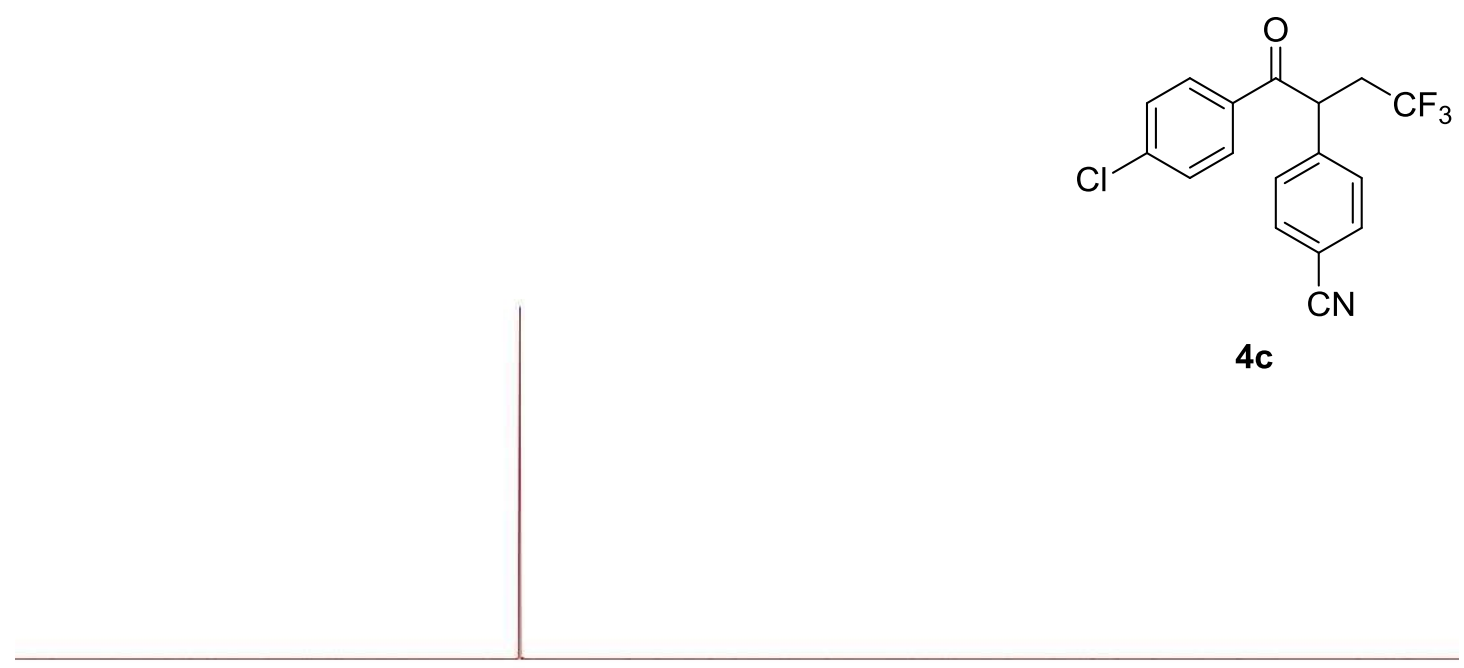




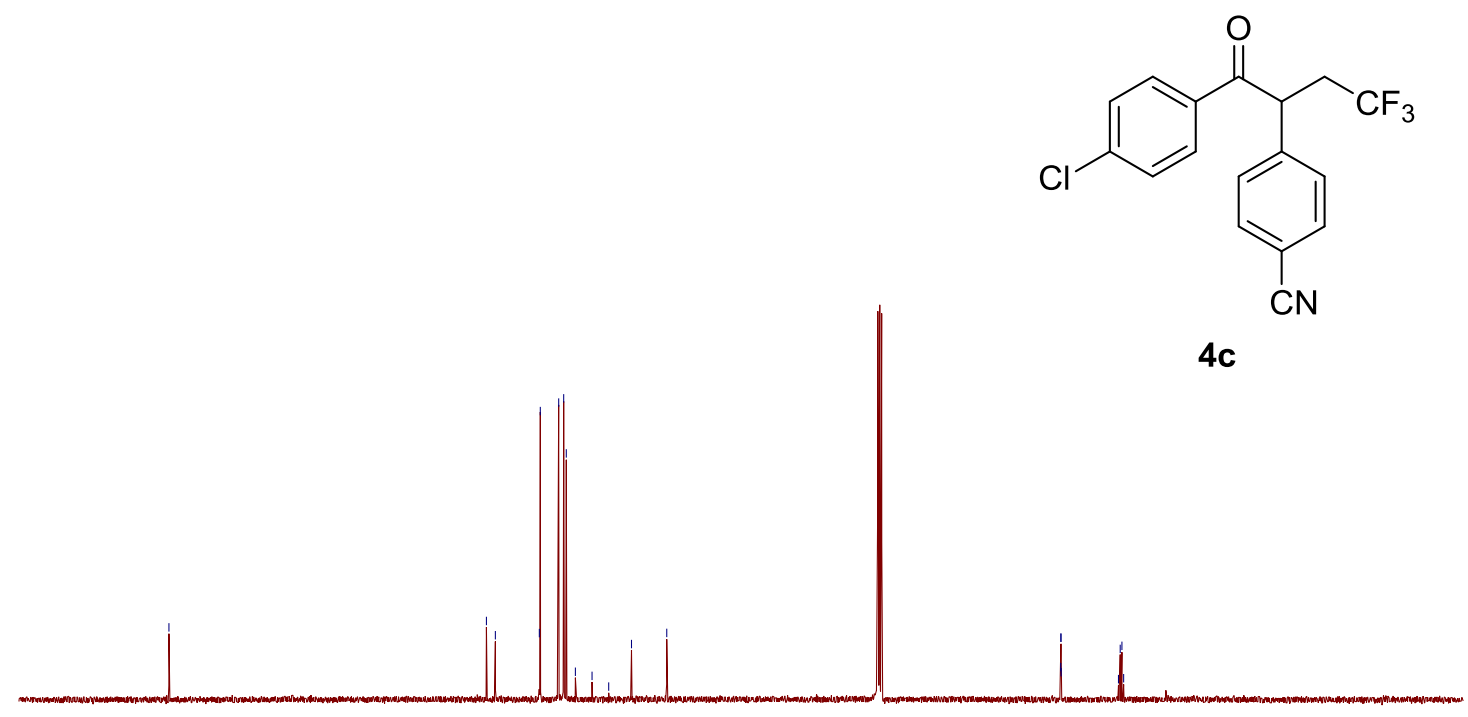




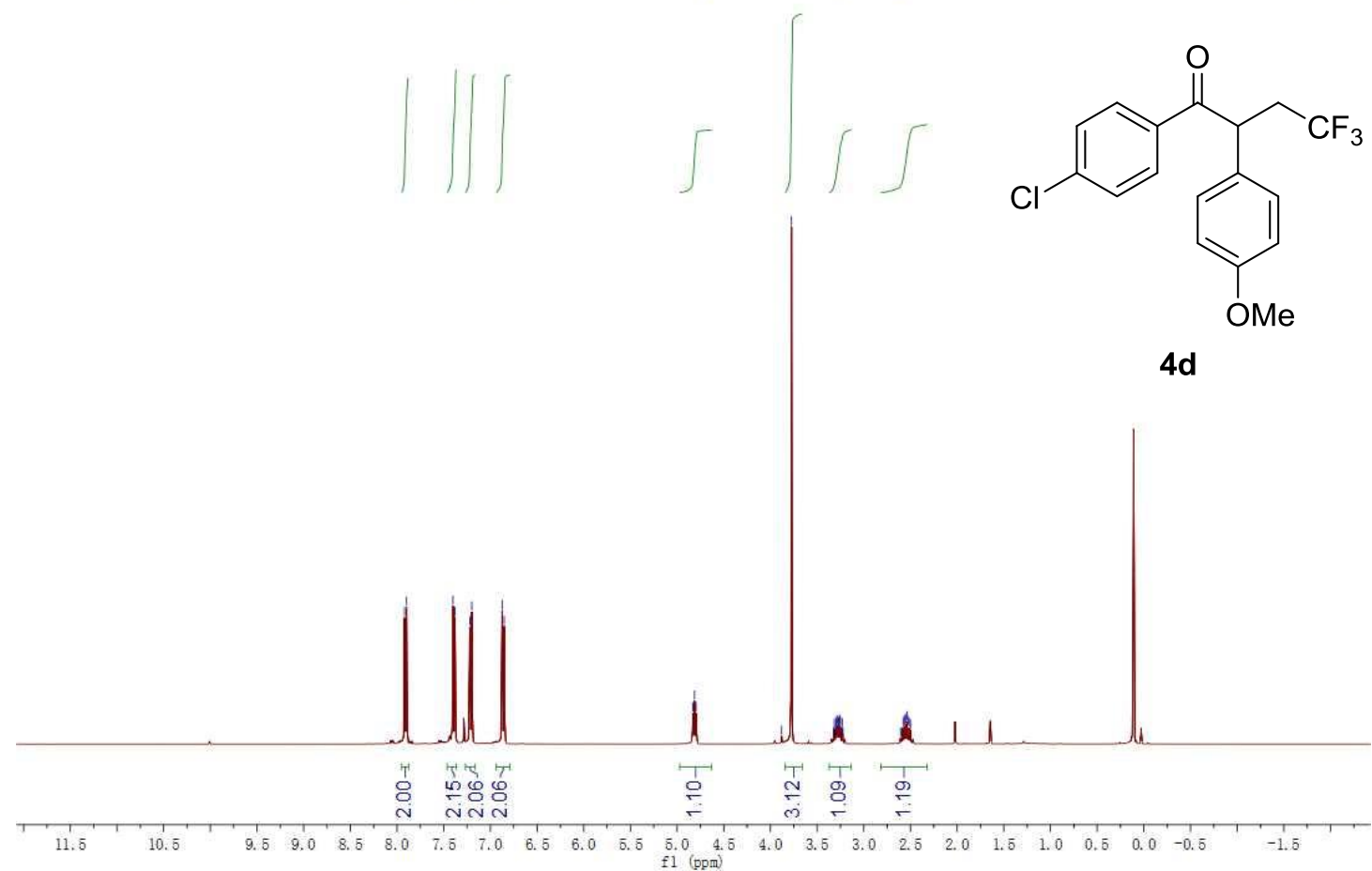

$191116-2 \mathrm{ZB}-2-13$

员<smiles>COc1ccc(C(CC(F)(F)F)C(=O)c2ccc(Cl)cc2)cc1</smiles>

4d

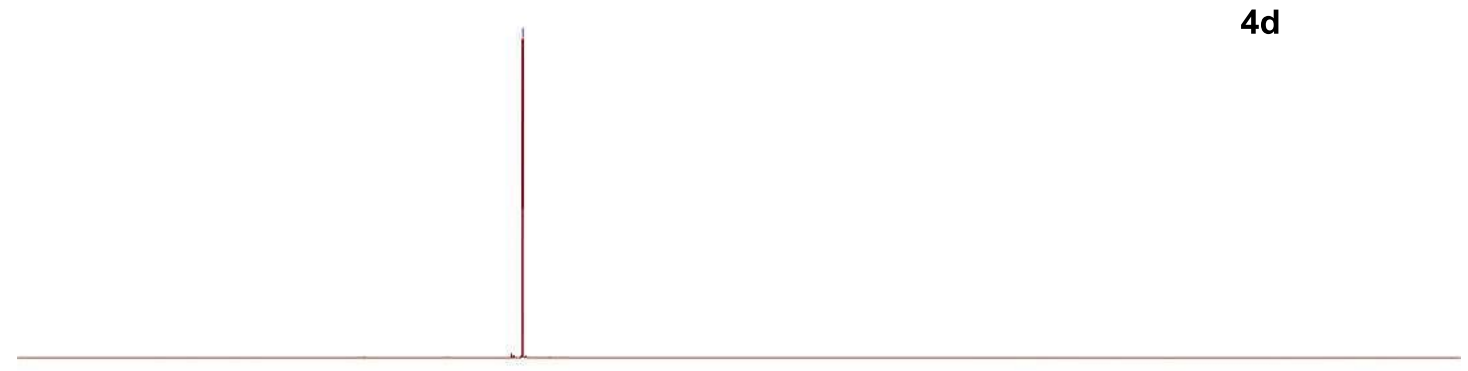

10
10 


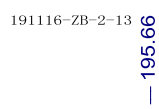

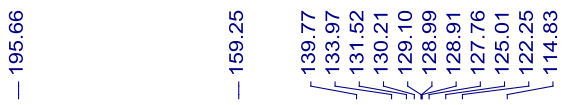

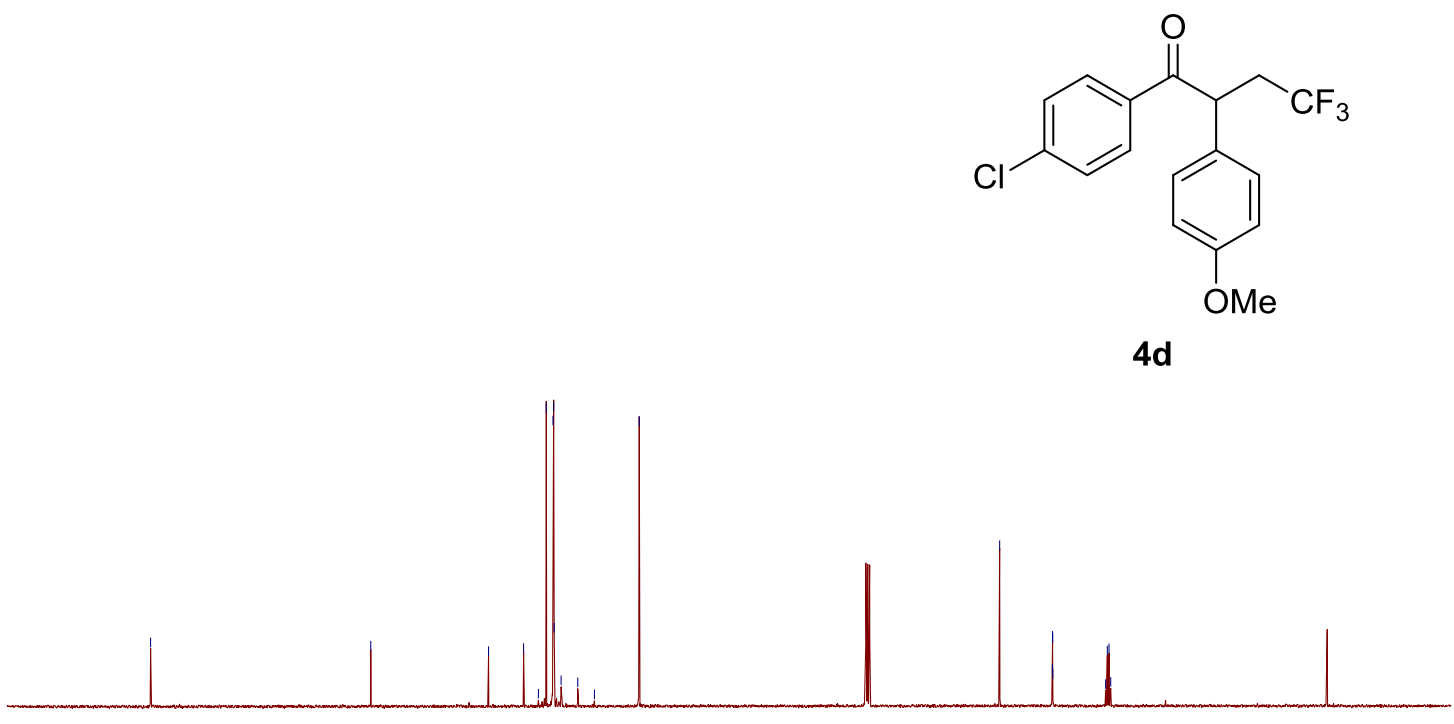

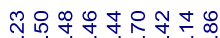

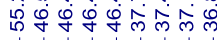

证

$4 d$

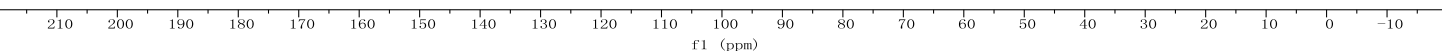




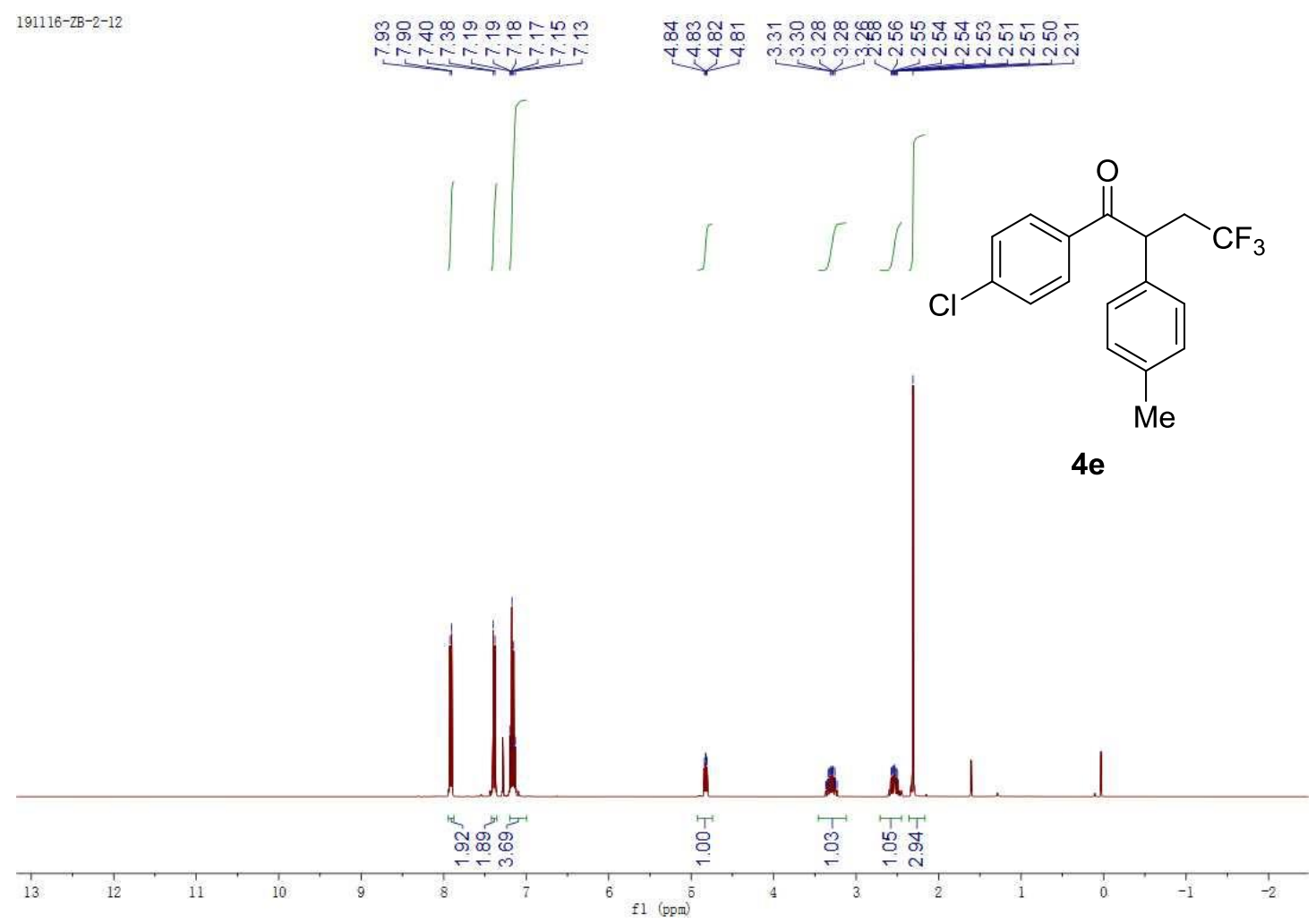

$191116-2 \mathrm{~B}-2-12$

ڤ̊<smiles>Cc1ccc(C(CC(F)(F)F)C(=O)c2ccc(Cl)cc2)cc1</smiles>

$4 e$

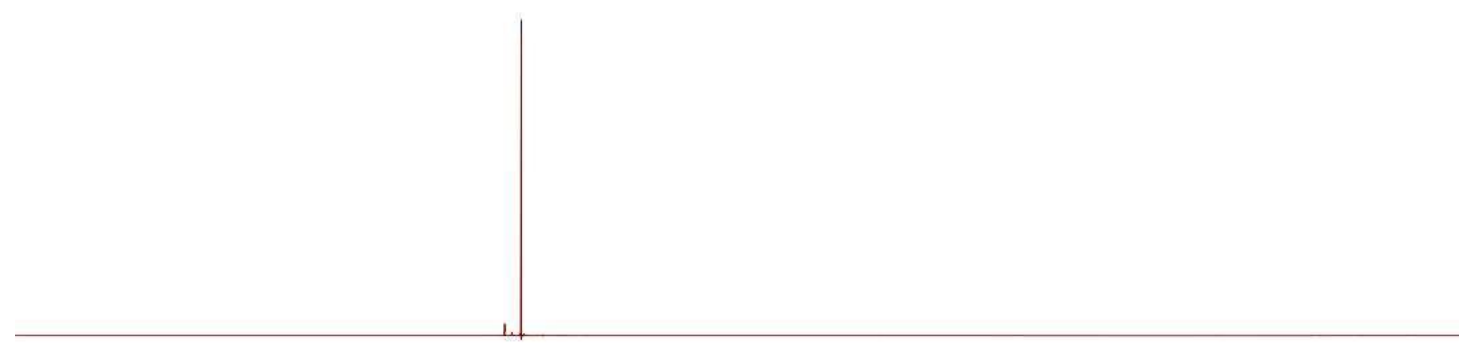




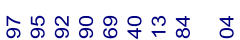

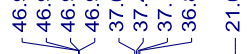
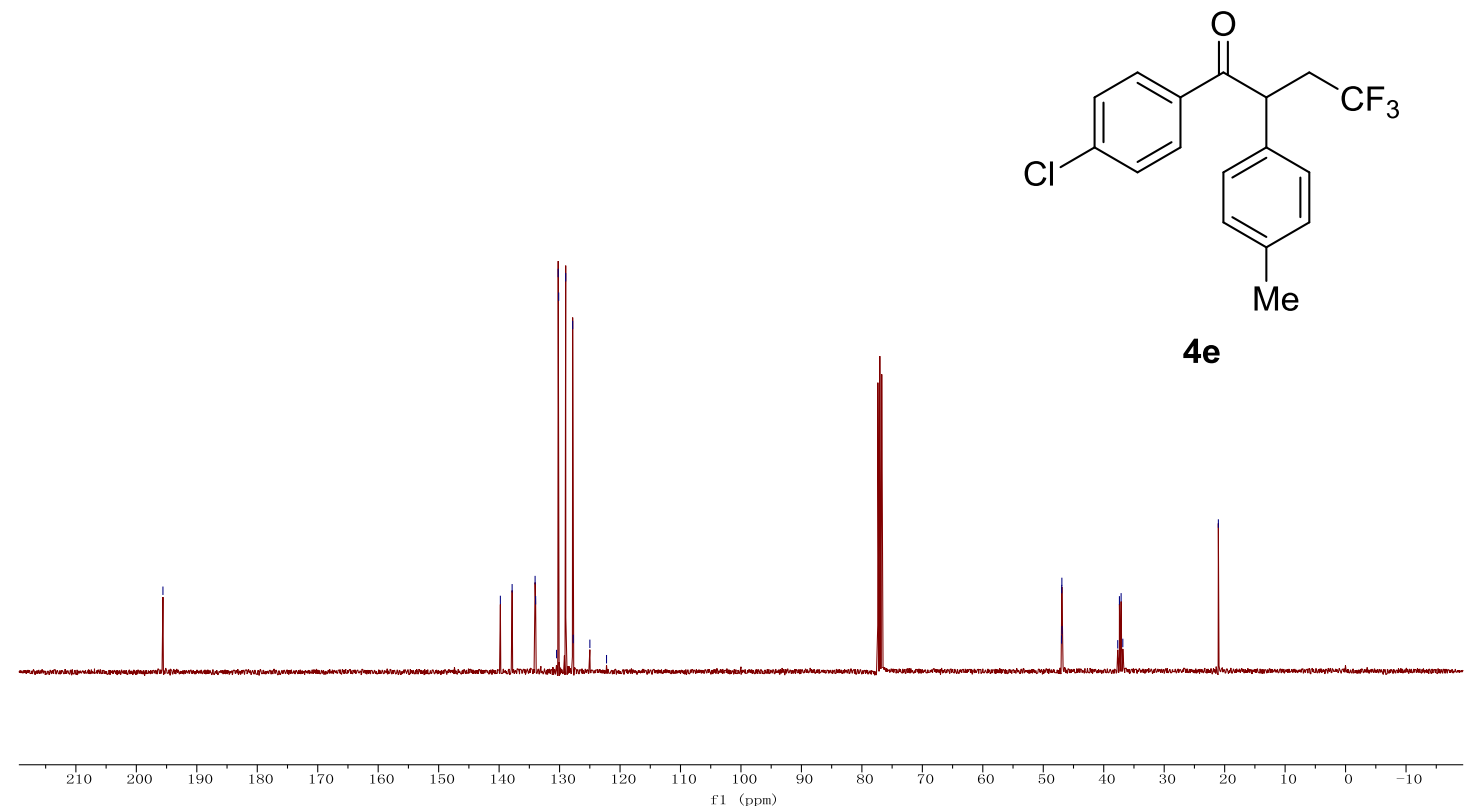

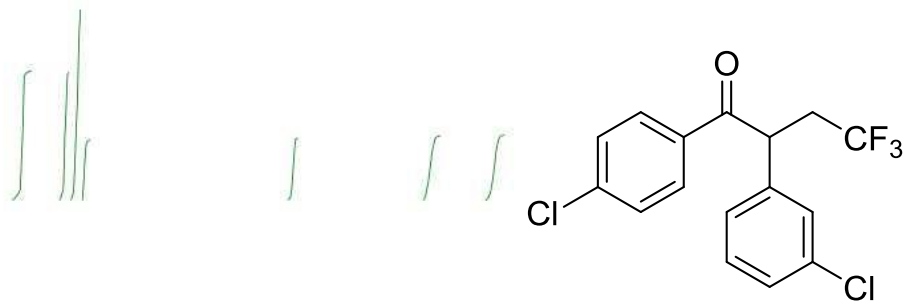

$4 f$

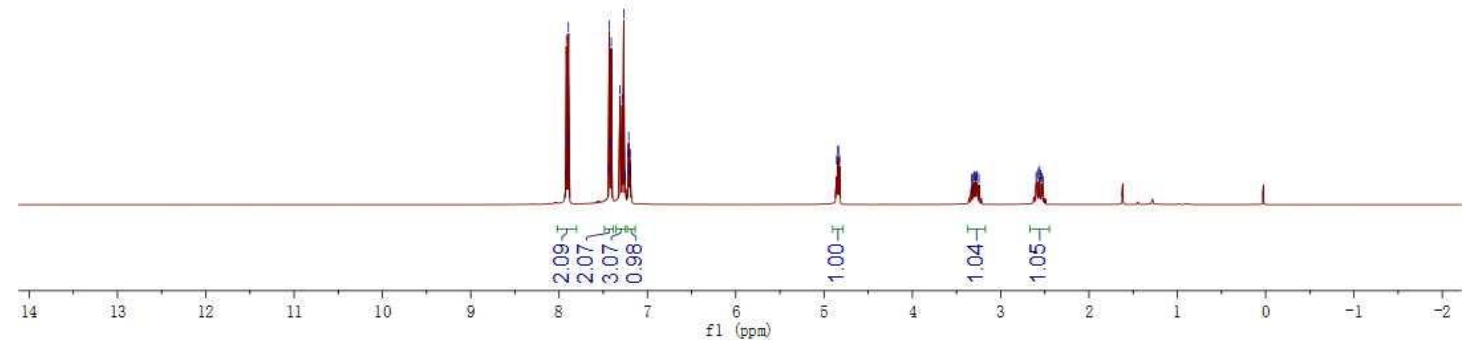

$191116-2 B-2-14$

怘<smiles>O=C(c1ccc(Cl)cc1)C(CC(F)(F)F)c1cccc(Cl)c1</smiles>

$4 f$

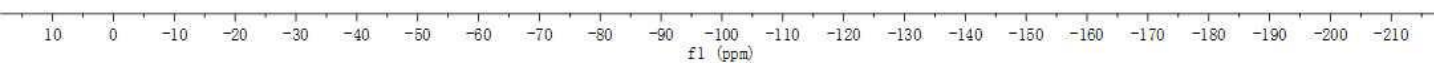



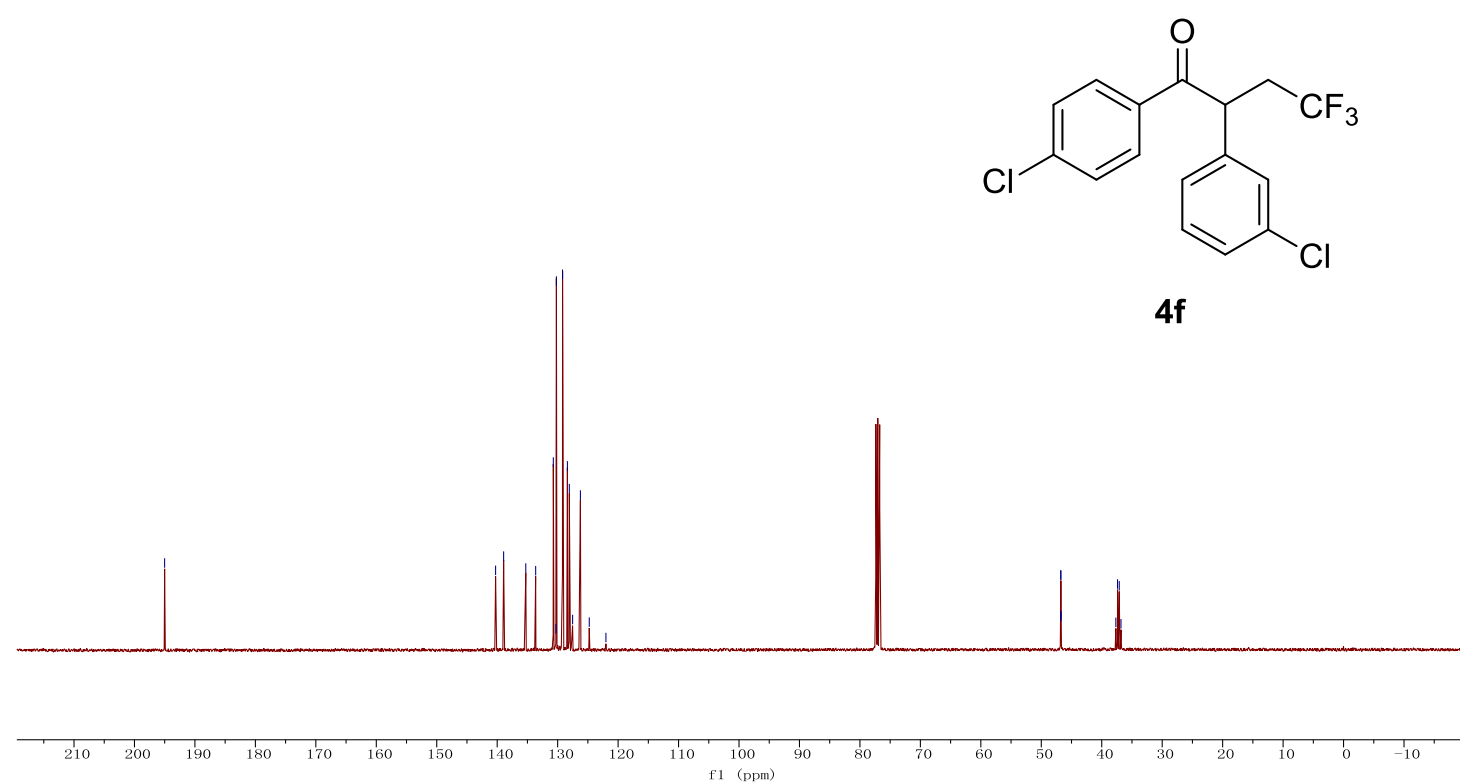


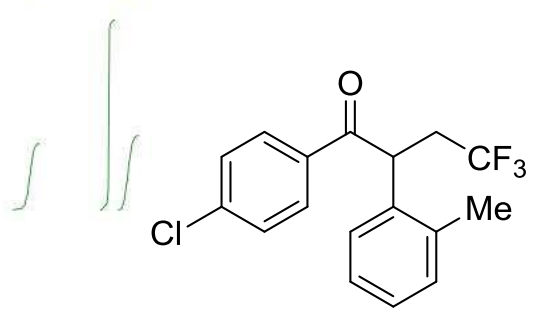

$4 g$

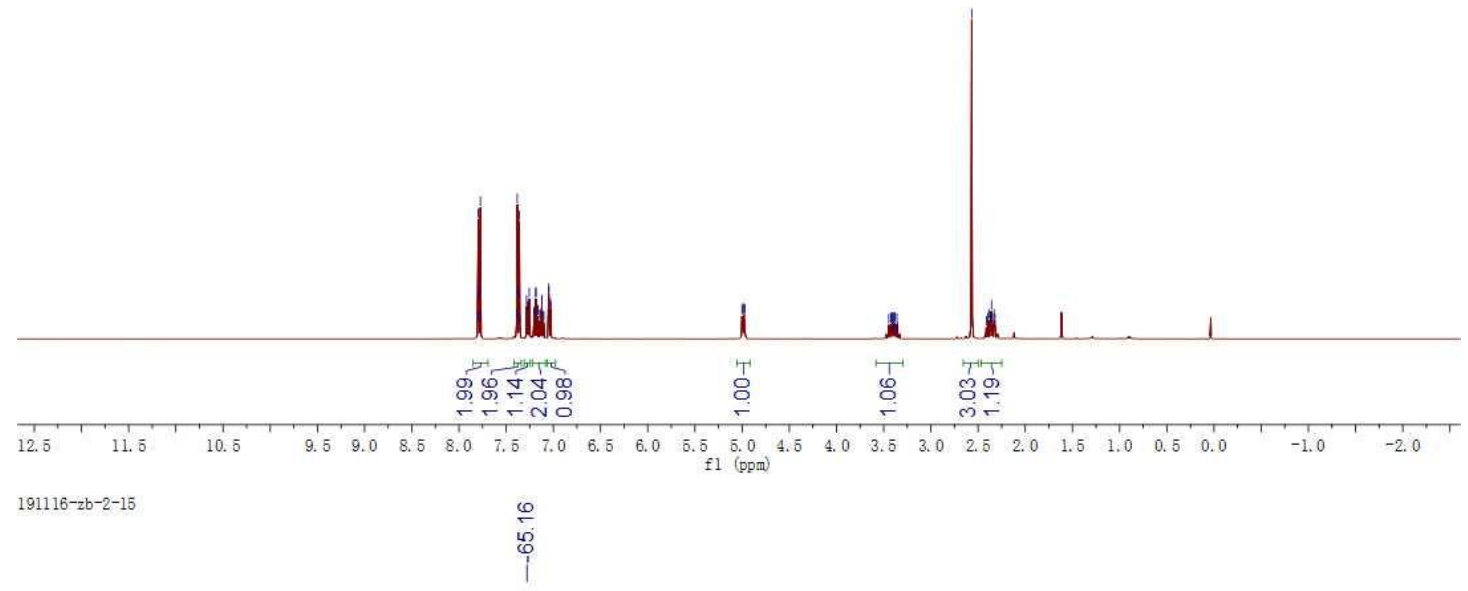<smiles>Cc1ccccc1C(CC(F)(F)F)C(=O)c1ccc(Cl)cc1</smiles>

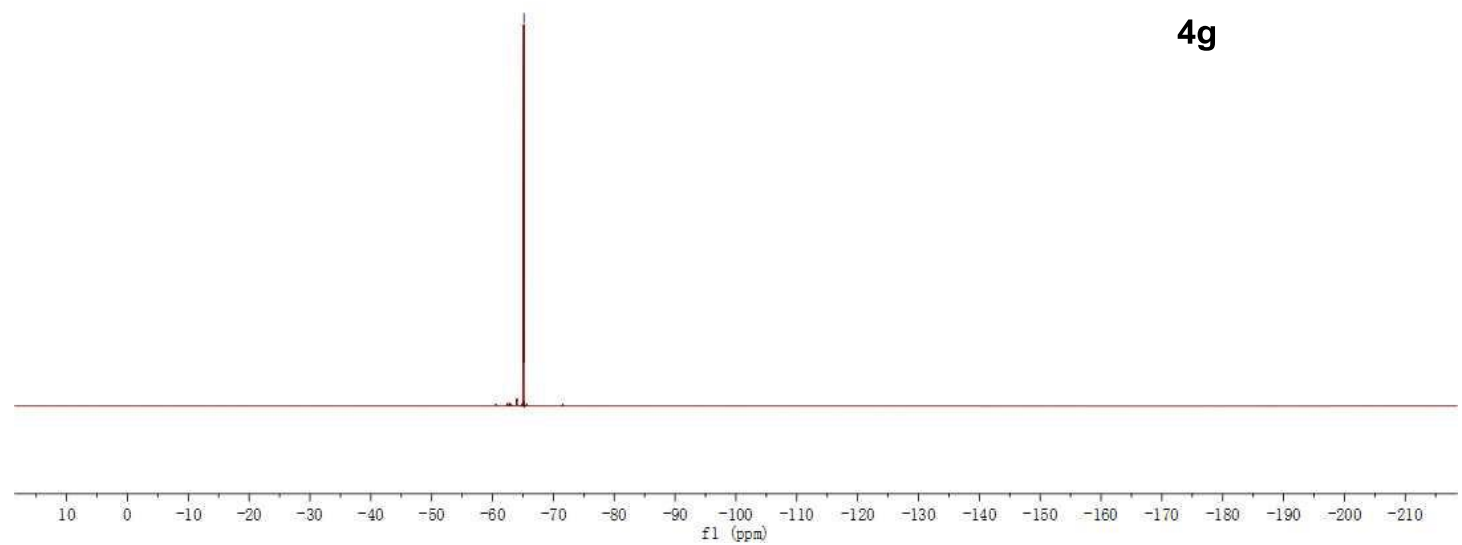


191116-zb-2-15

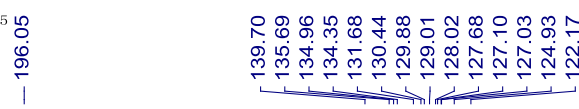

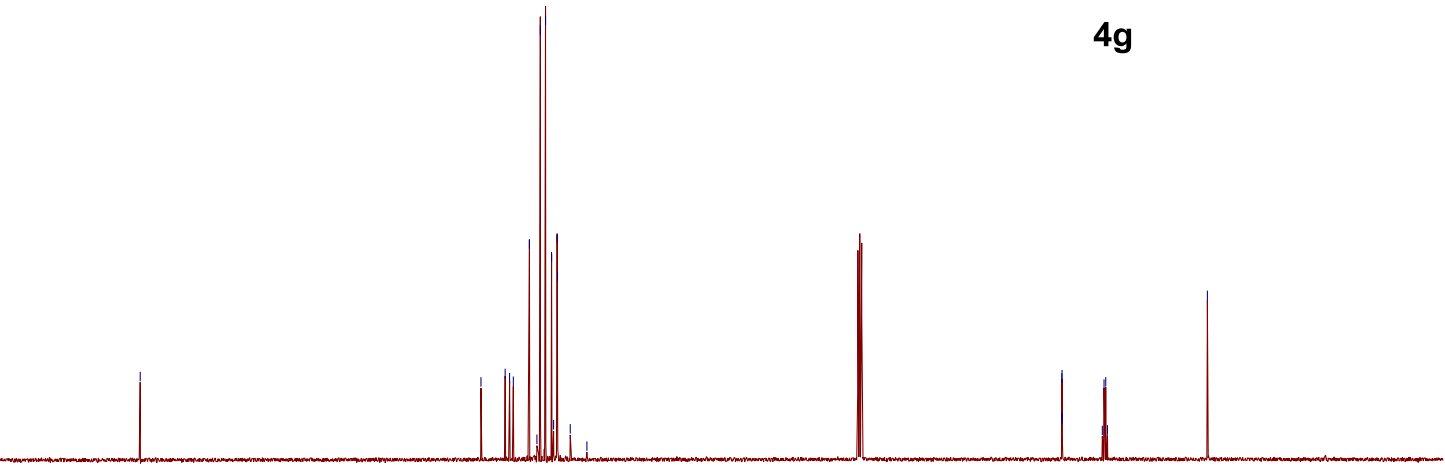

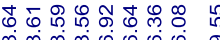

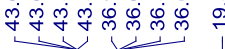

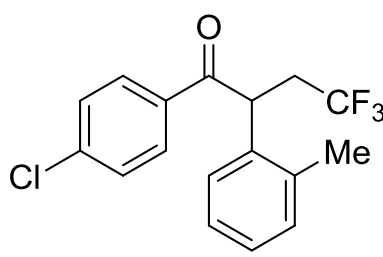

$4 \mathrm{~g}$

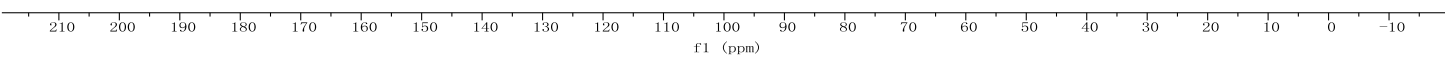




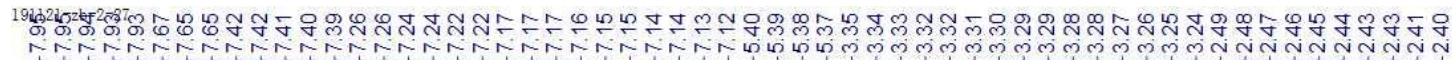

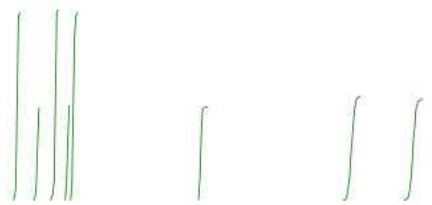<smiles>O=C(c1ccc(Cl)cc1)C(CC(F)(F)F)c1ccccc1Br</smiles>

4h

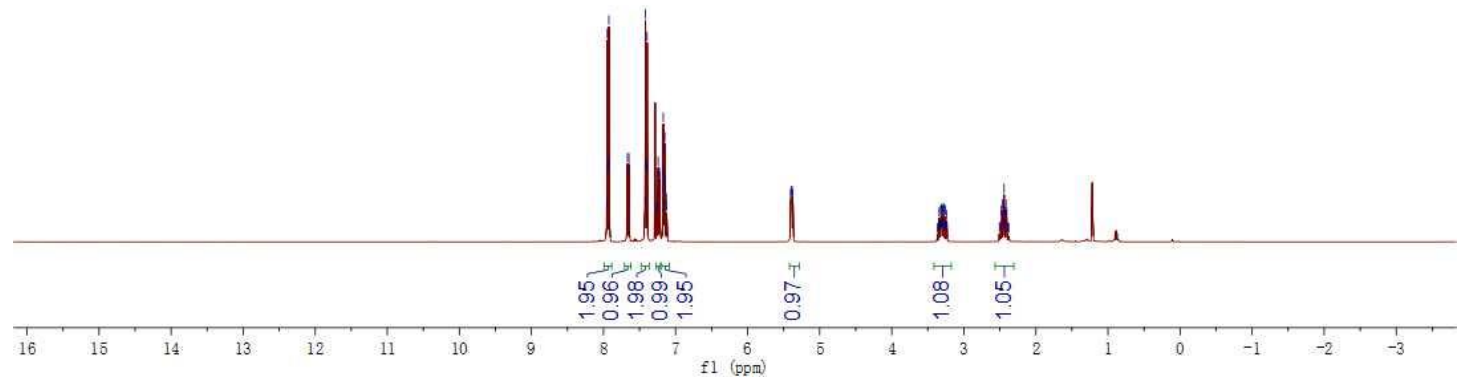

$191121-z b-2-27$

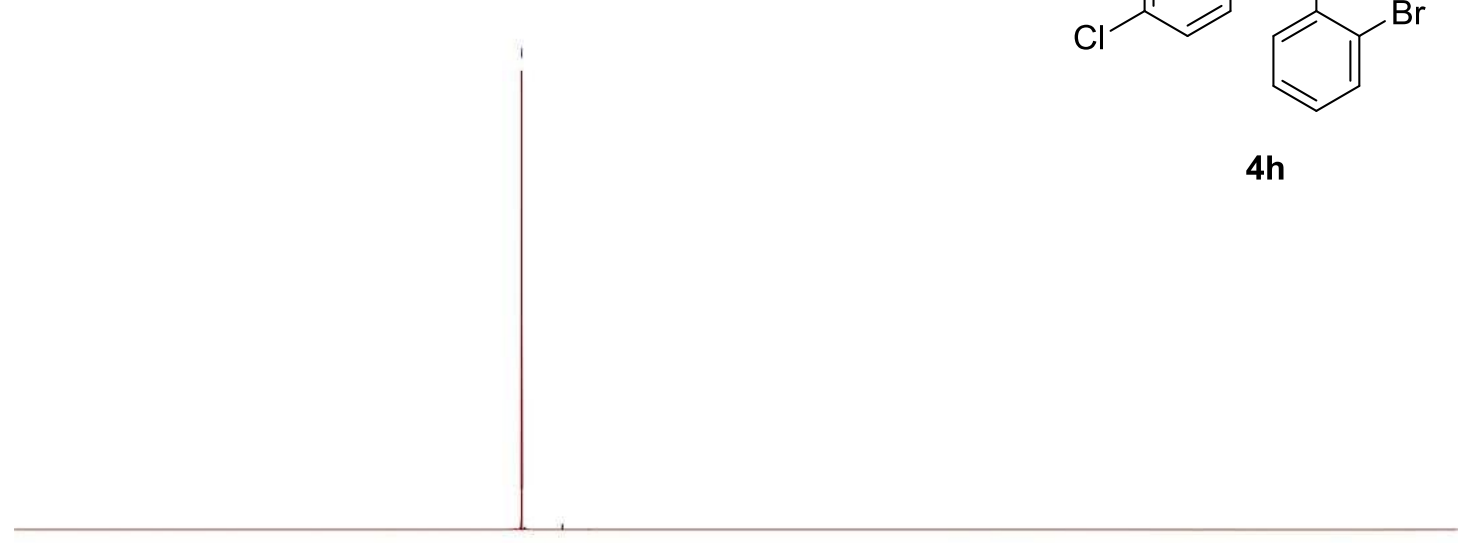<smiles>O=C(c1ccc(Cl)cc1)C(CC(F)(F)F)c1ccccc1Br</smiles>

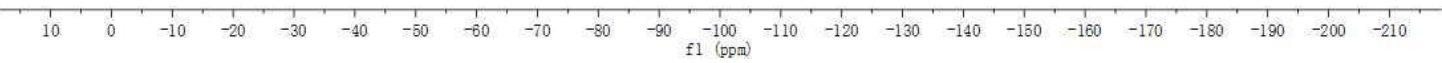




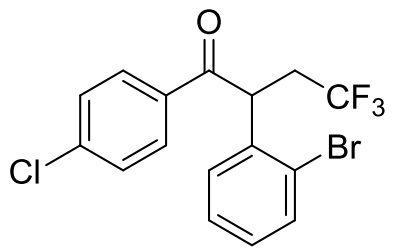

4h

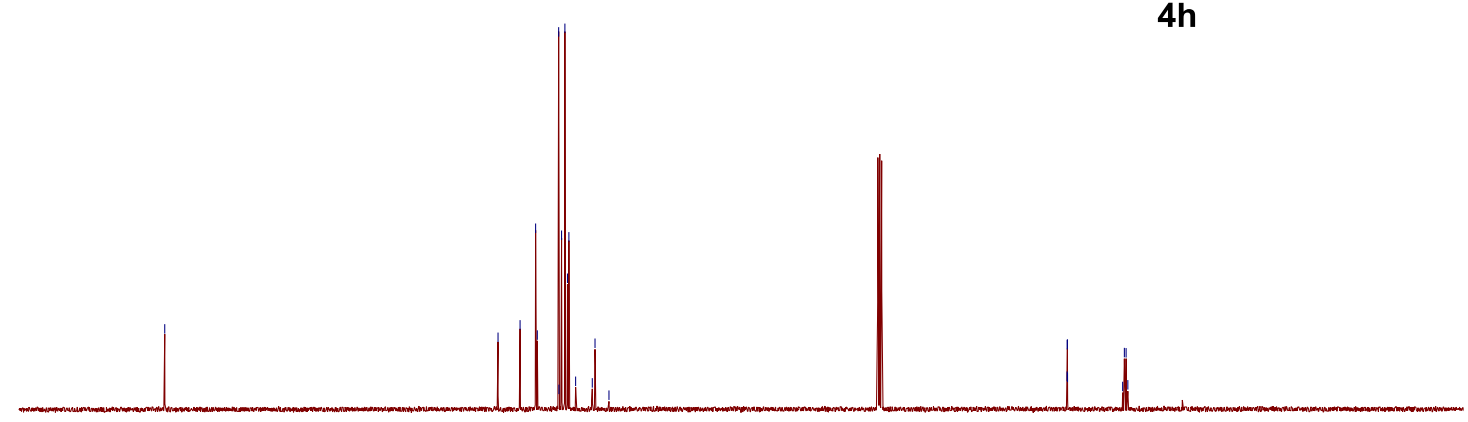

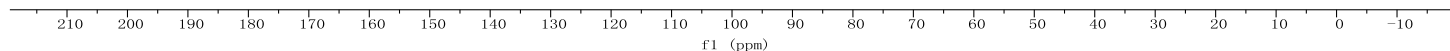




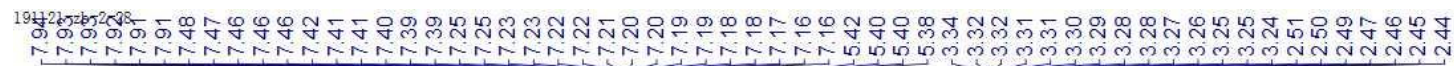

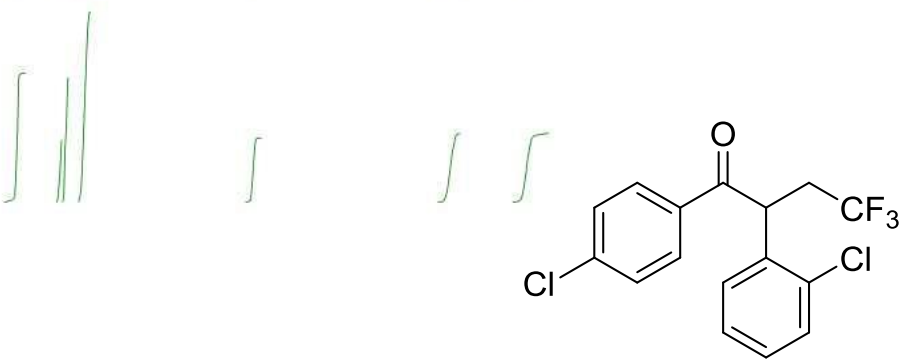

$4 i$

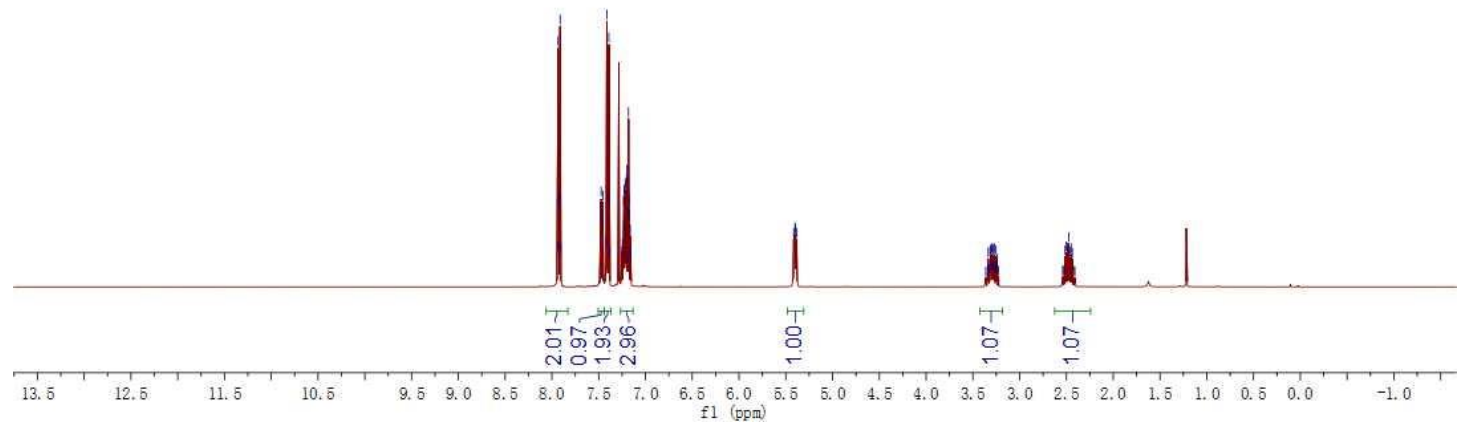

$191121-z b-2-28$

$\infty$
$\infty$
0
0
1

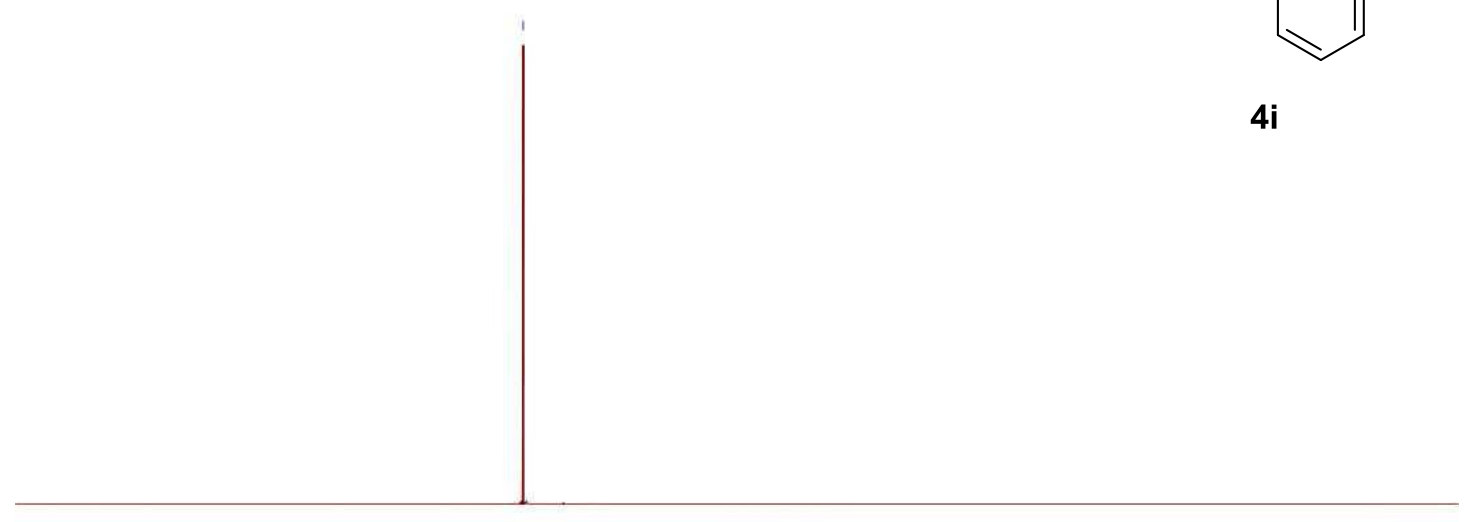<smiles>O=C(c1ccc(Cl)cc1)C(CC(F)(F)F)c1ccccc1Cl</smiles>

$4 i$

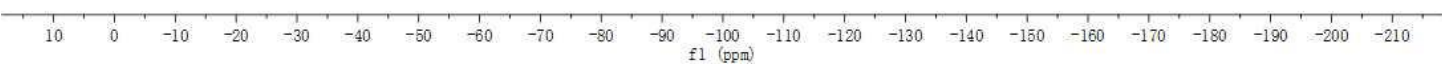




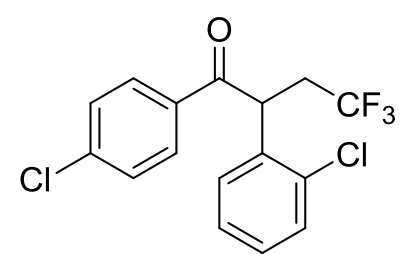

$4 i$

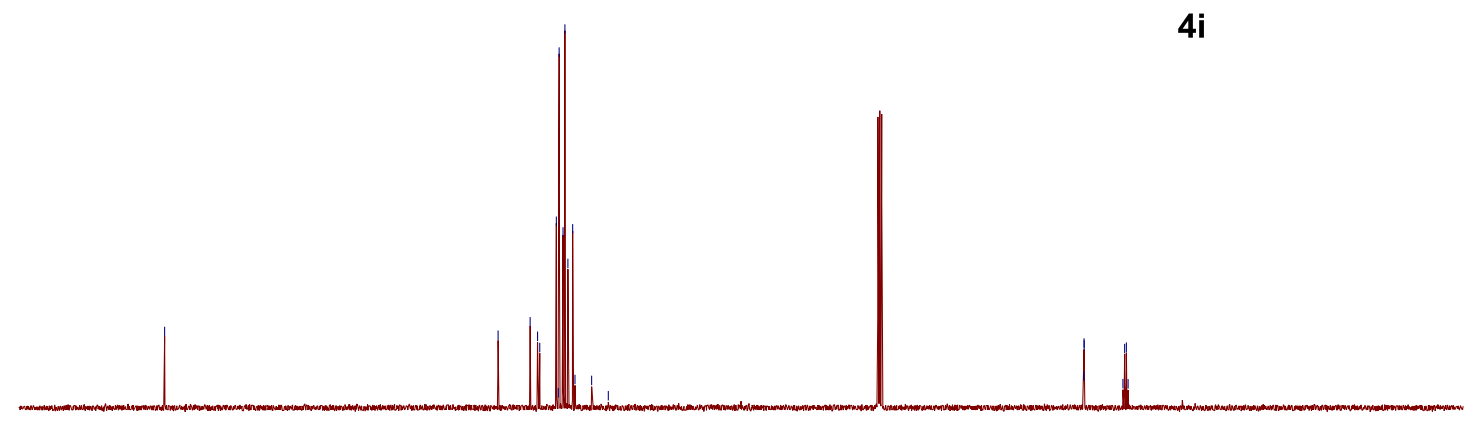

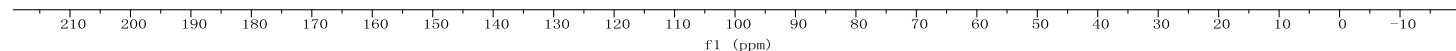




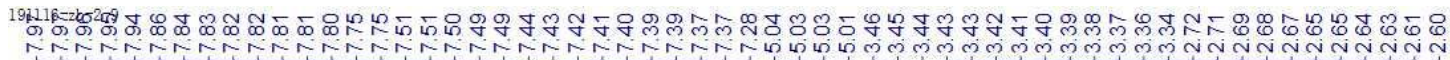

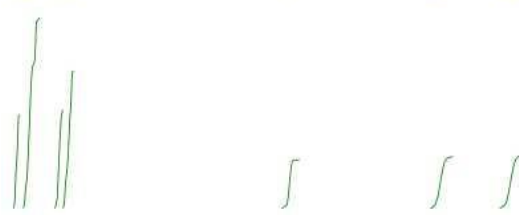<smiles>O=C(c1ccc(Cl)cc1)C(CC(F)(F)F)c1cccc2ccccc12</smiles>

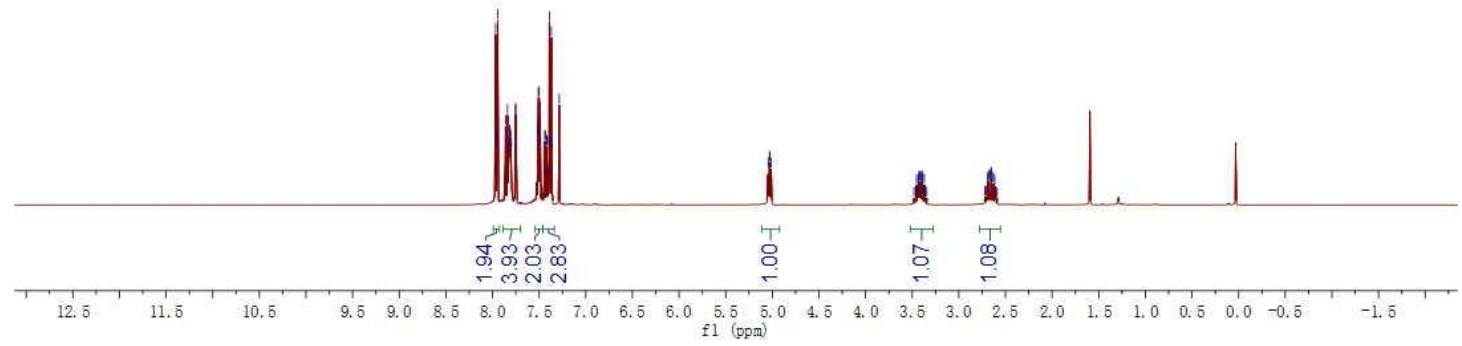

$191116-z b-2-9$

i<smiles>O=C(c1ccc(Cl)cc1)C(CC(F)(F)F)c1cccc2ccccc12</smiles>

4j

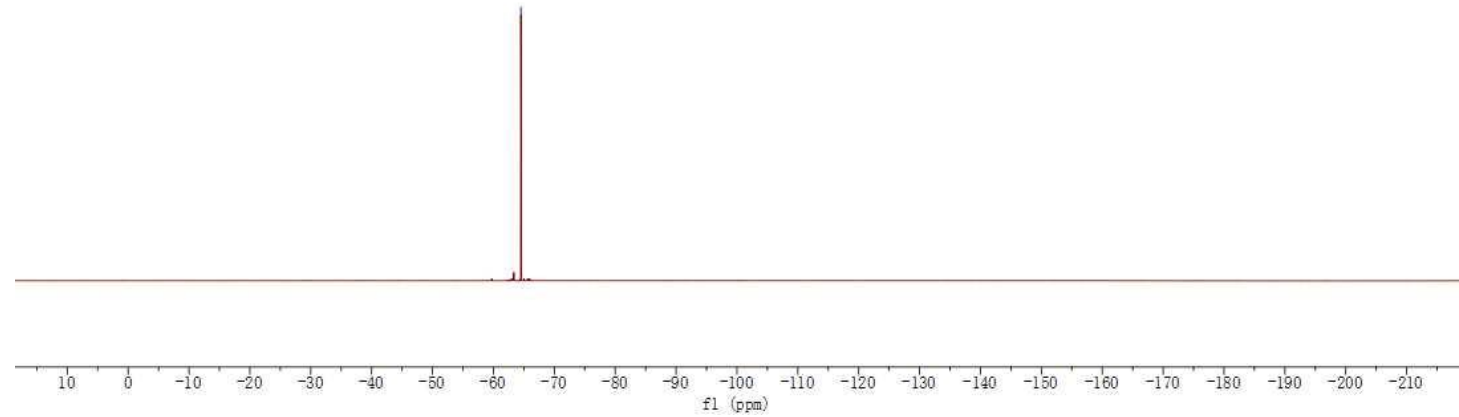




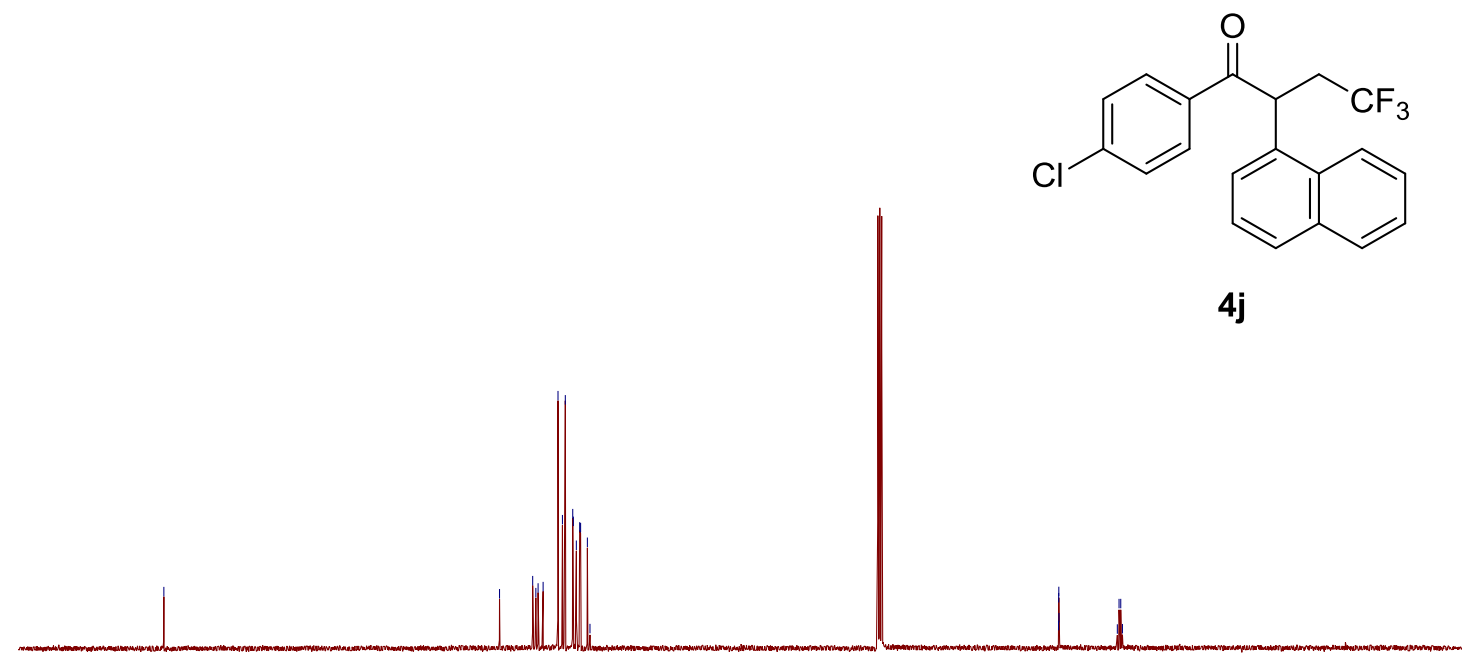

4j

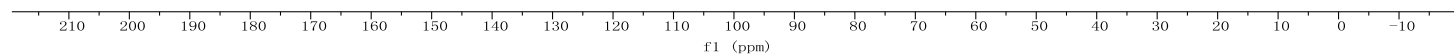




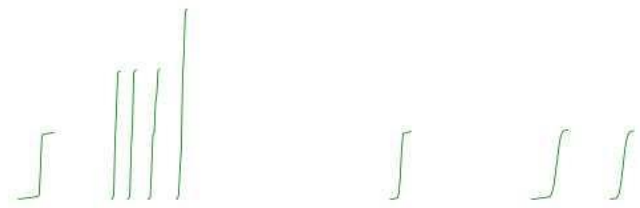<smiles>O=C(c1ccc(Cl)cc1)C(CC(F)(F)F)c1ccc2ncccc2c1</smiles>

$4 k$

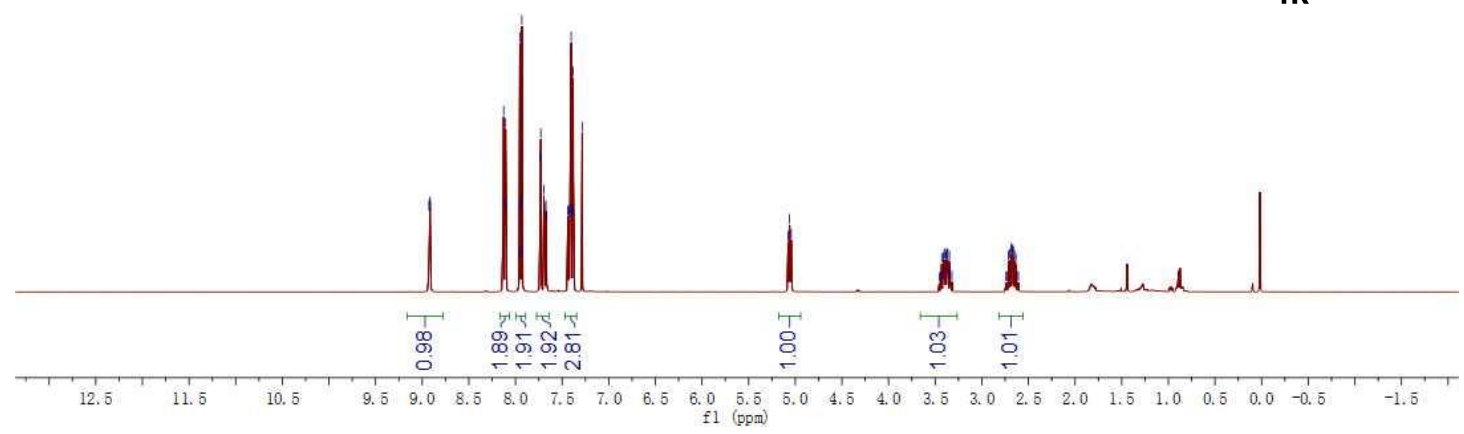

20191117-ZB-2-3. 13. fid<smiles>O=C(c1ccc(Cl)cc1)C(CC(F)(F)F)c1ccc2ncccc2c1</smiles>

4k 

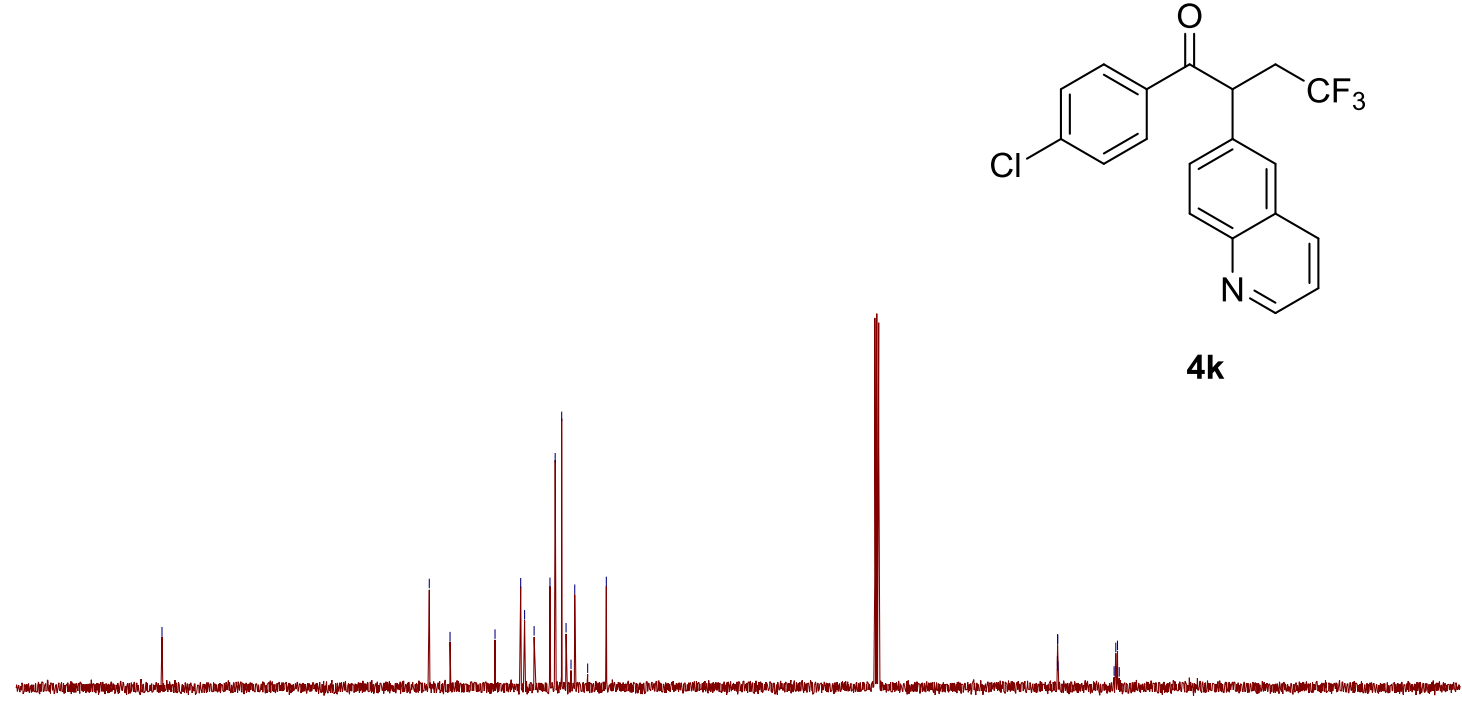

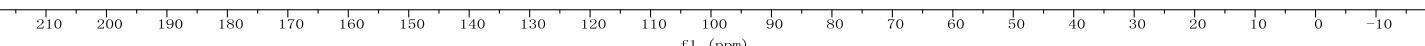


कొ

No
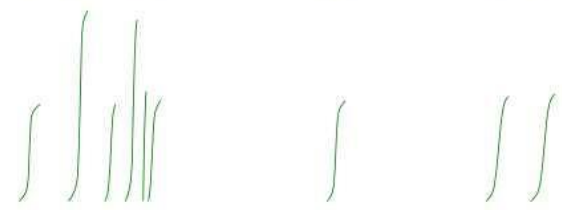<smiles>O=C(c1ccc(Cl)cc1)C(CC(F)(F)F)c1ccccn1</smiles>

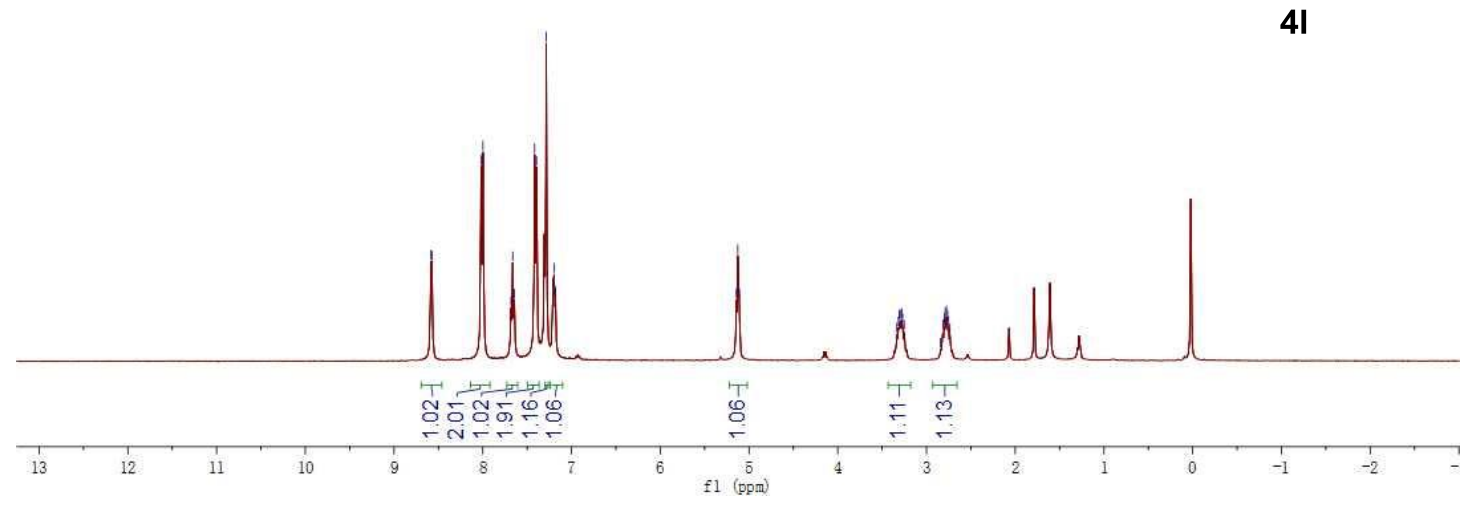

191119-2B-2-1-true

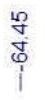
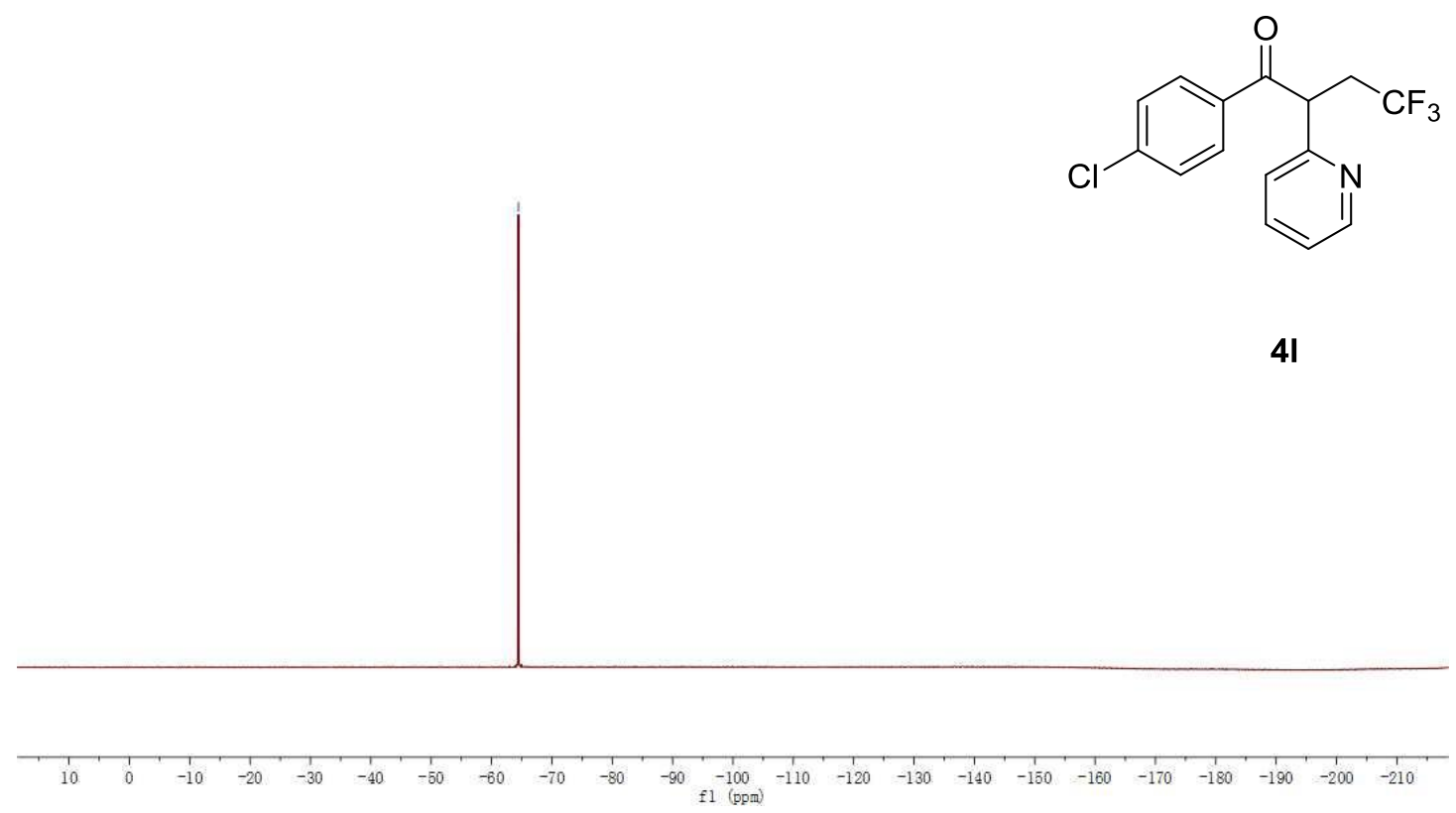

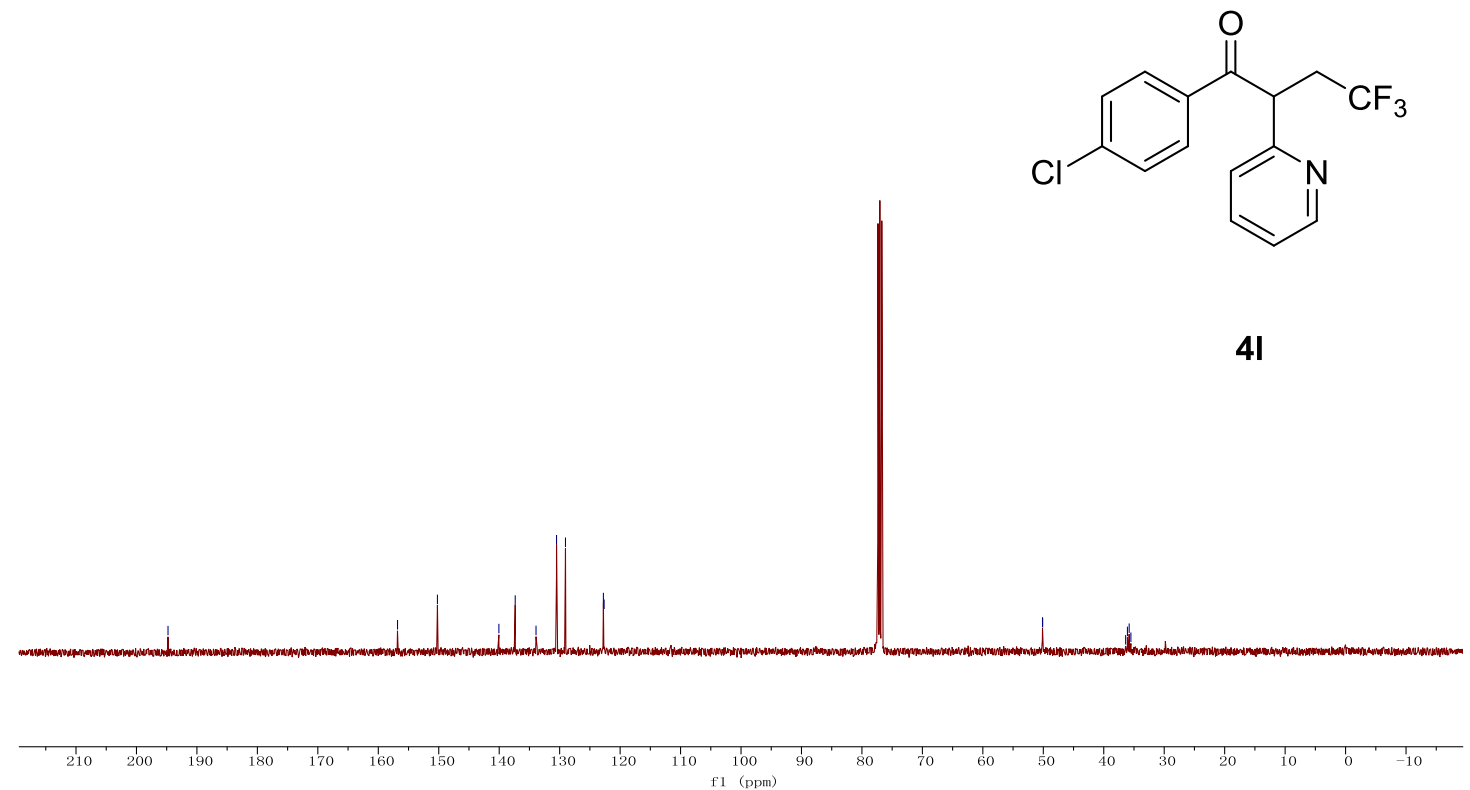
$191116-z b-2-8$
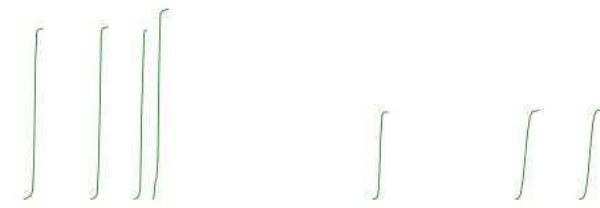<smiles>O=C(c1ccc(Cl)cc1)C(CC(F)(F)F)c1ccncc1</smiles>

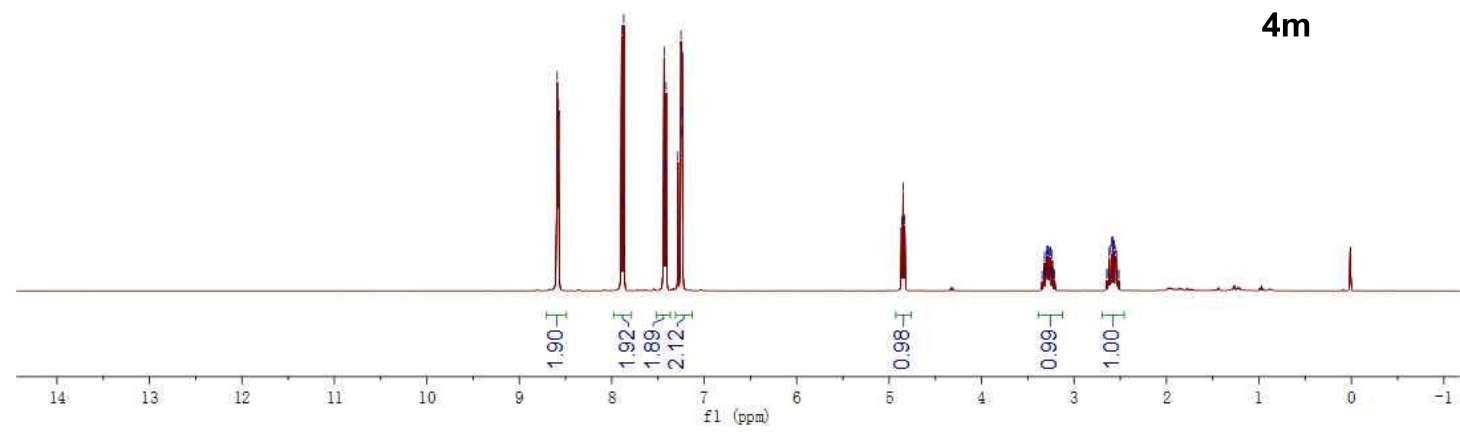

$191116-z b-2-8$

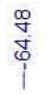

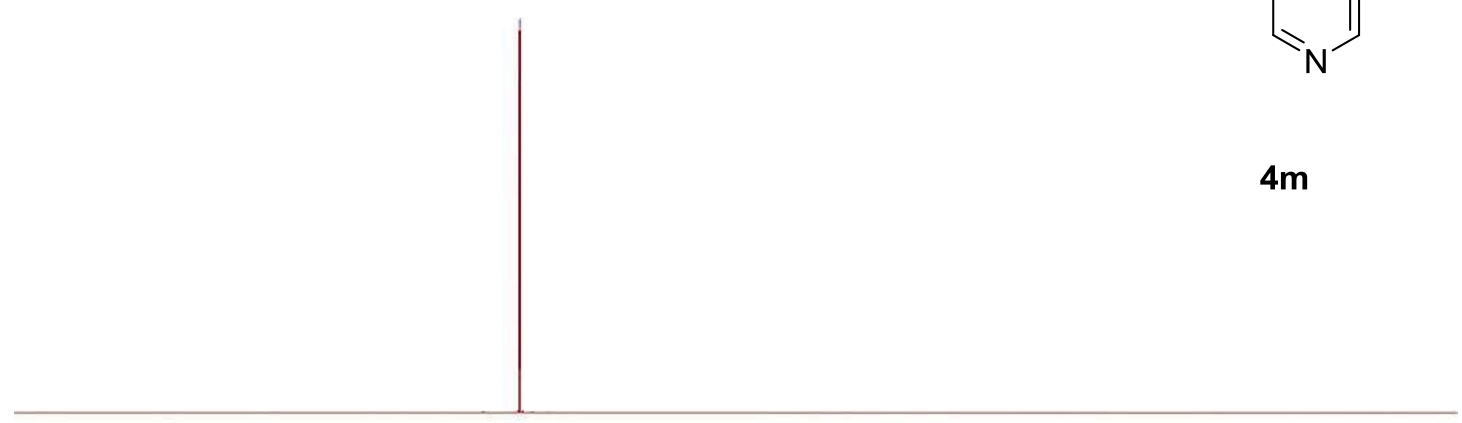<smiles>O=C(c1ccc(Cl)cc1)C(CC(F)(F)F)c1ccncc1</smiles> 
<smiles>O=C(c1ccc(Cl)cc1)C(CC(F)(F)F)c1ccncc1</smiles>
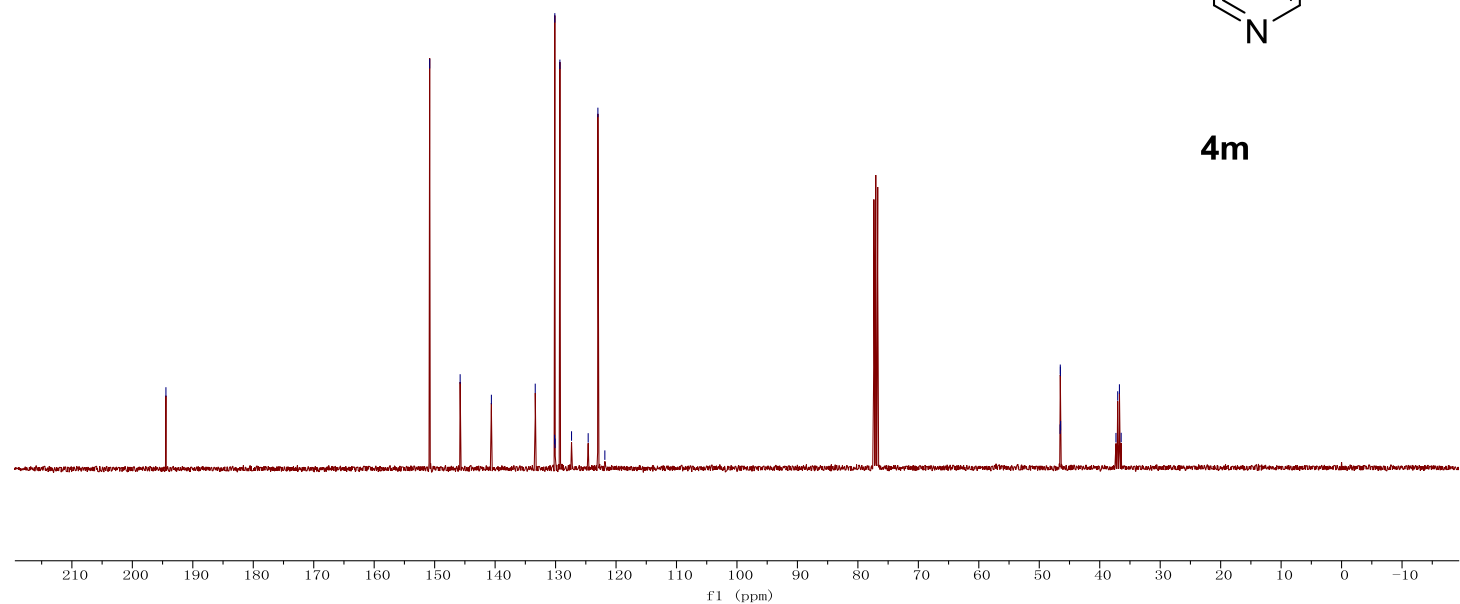

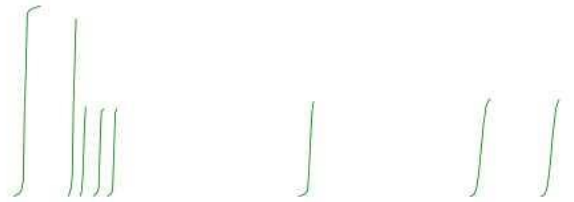<smiles>O=C(c1ccc(Cl)cc1)C(CC(F)(F)F)c1ccsc1</smiles>

$4 n$

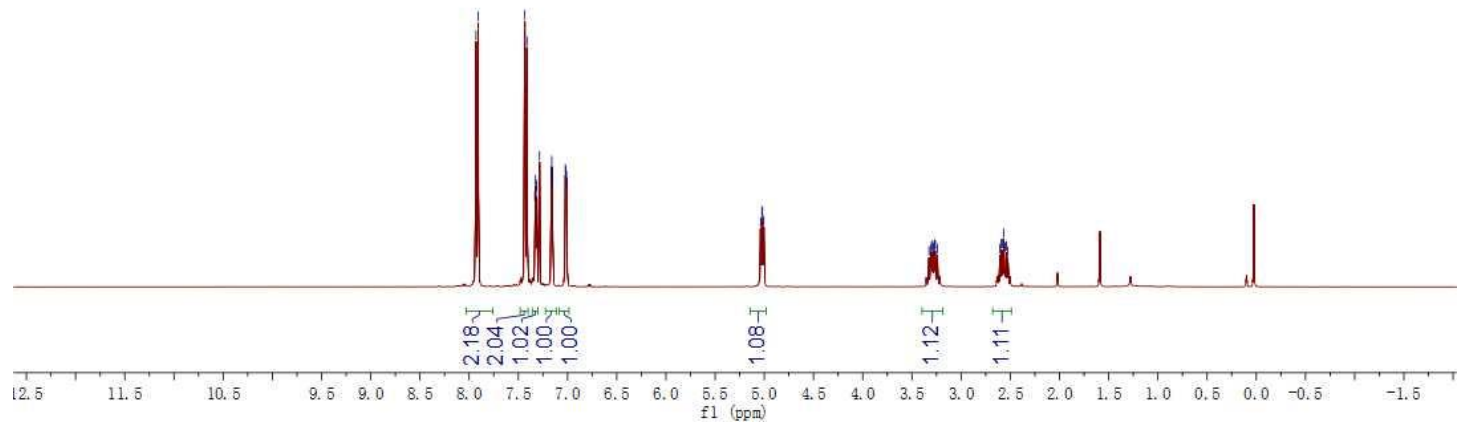
$191117-z b-2-5$<smiles>O=C(c1ccc(Cl)cc1)C(CC(F)(F)F)c1ccsc1</smiles>

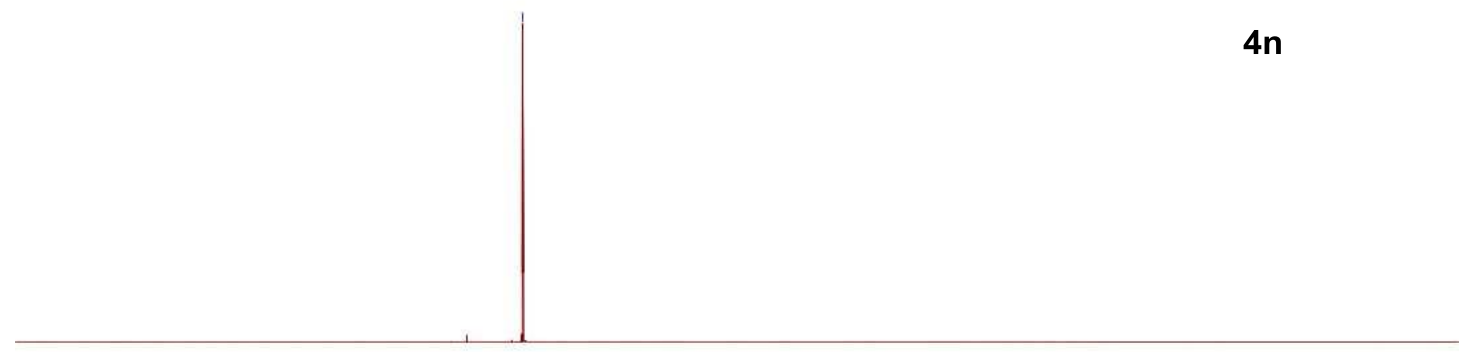


<smiles>O=C(c1ccc(Cl)cc1)C(CC(F)(F)F)c1ccsc1</smiles>

$4 n$

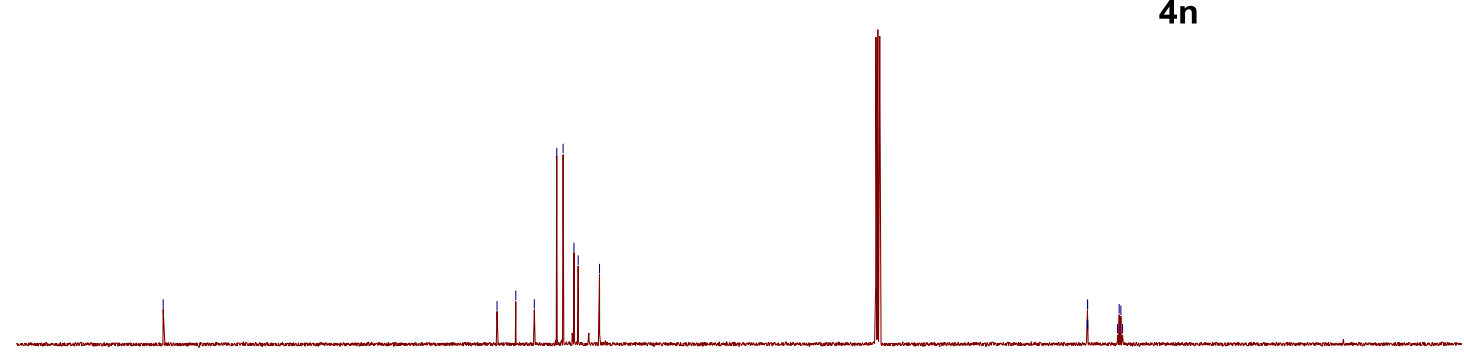

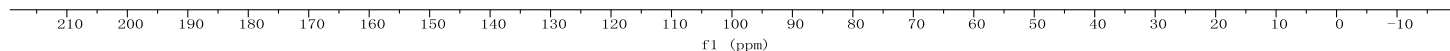



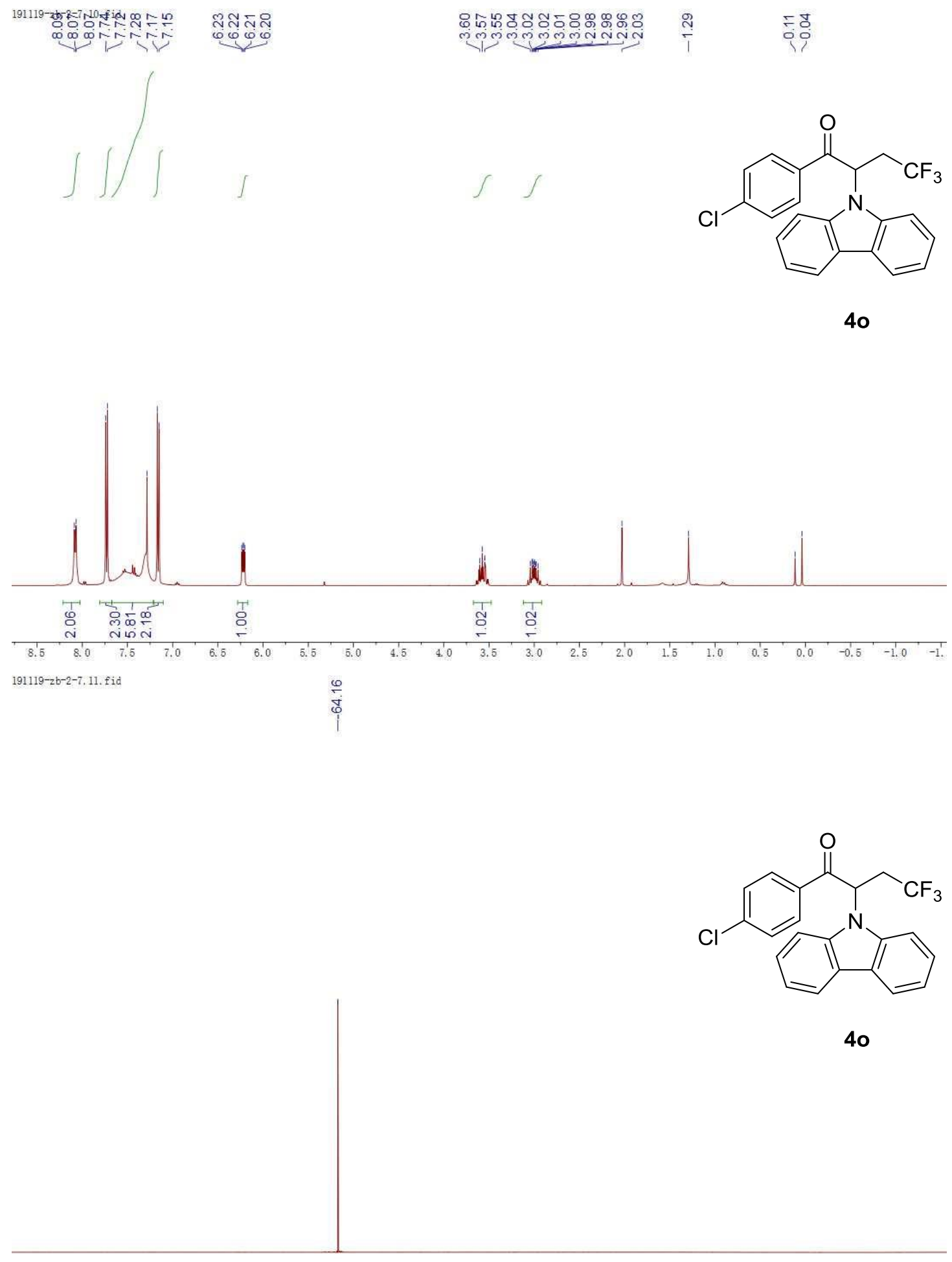

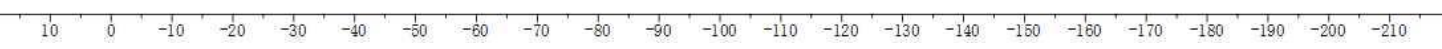




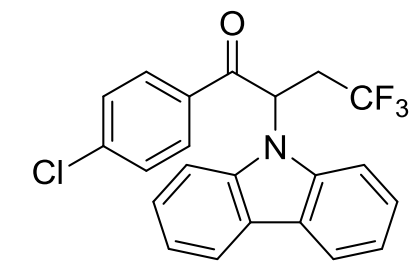

4o

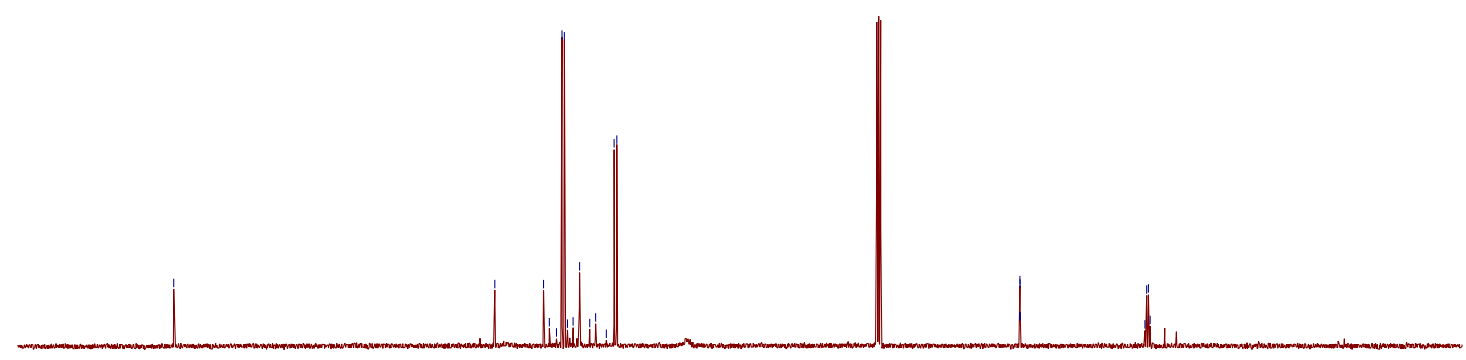



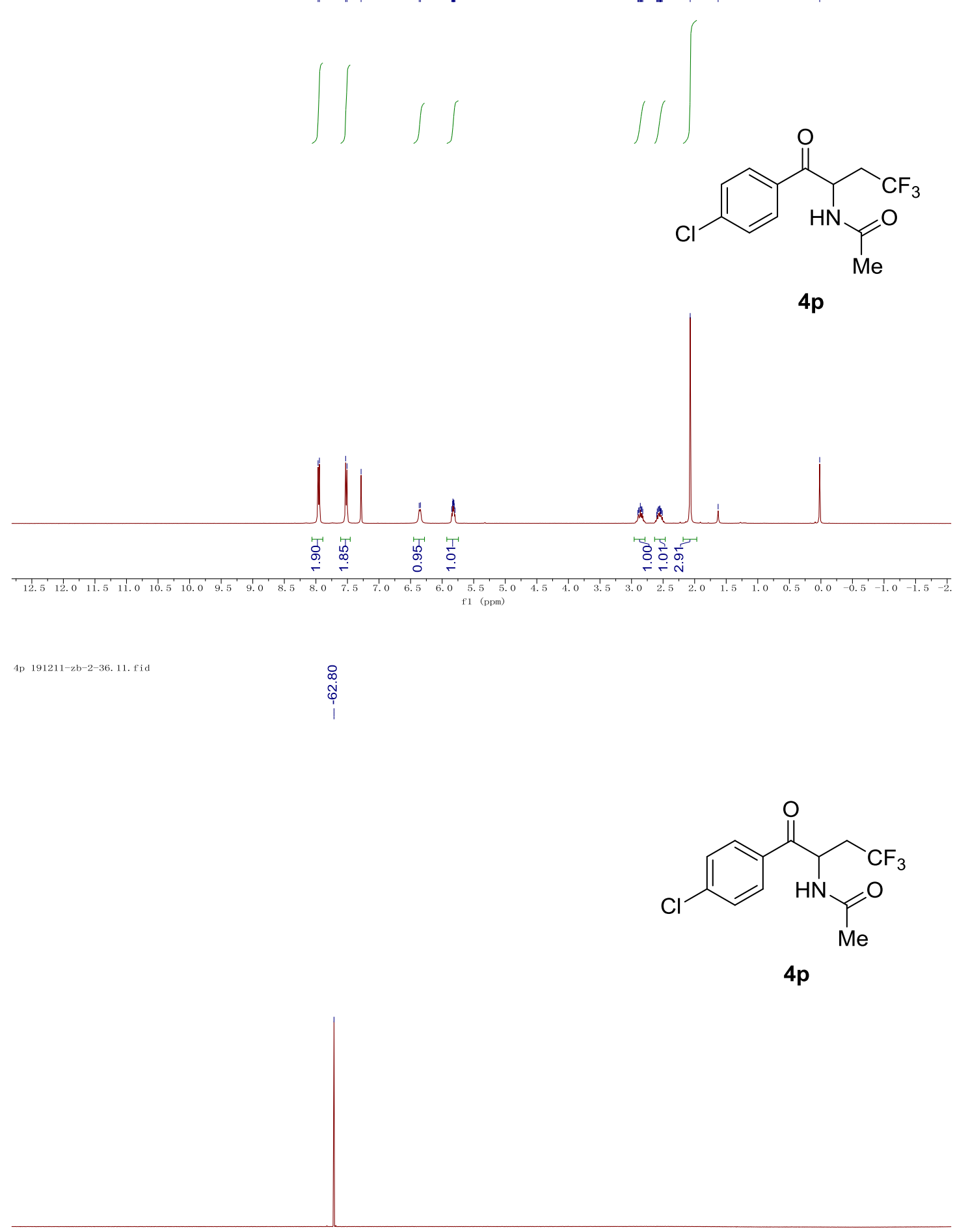

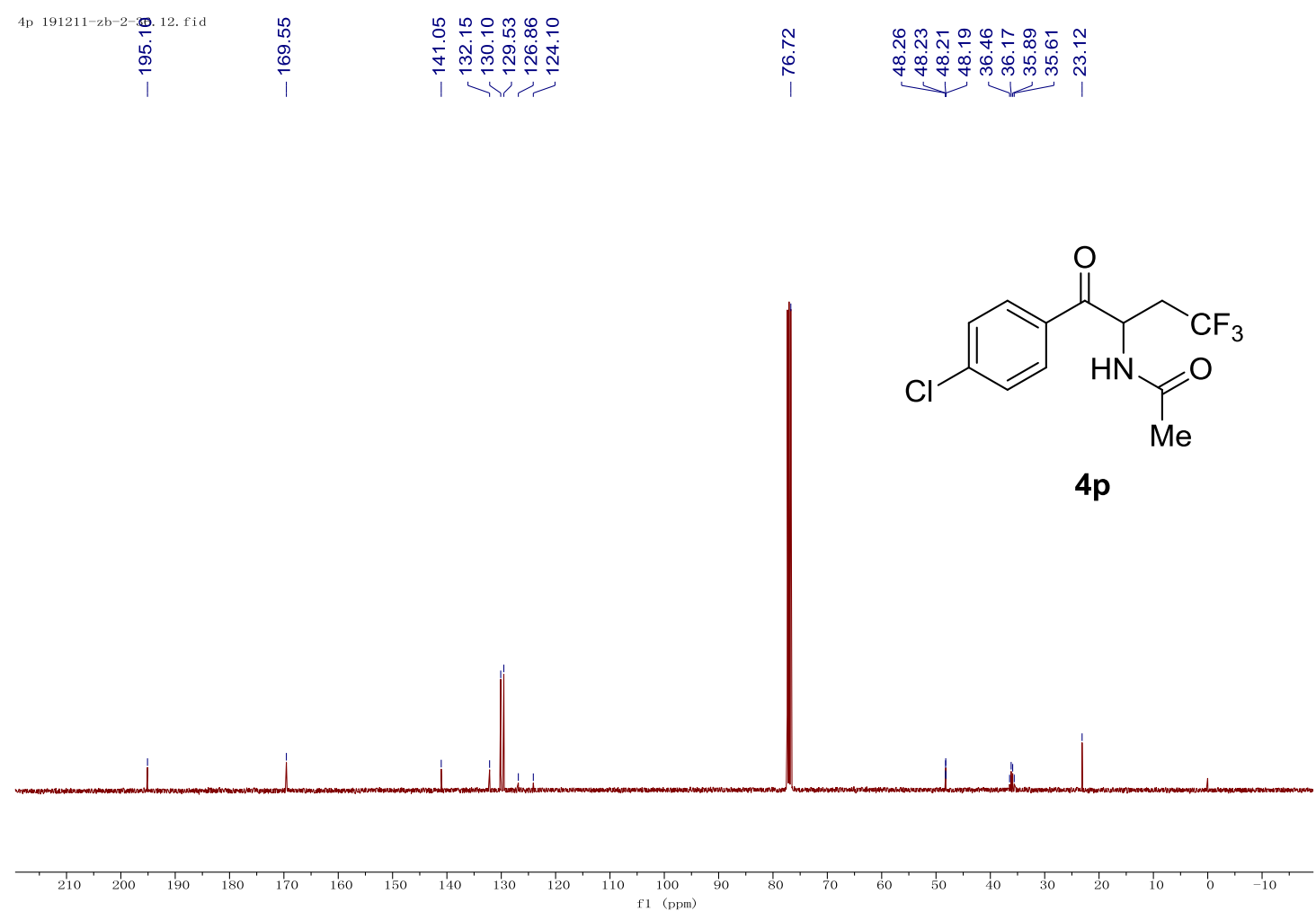

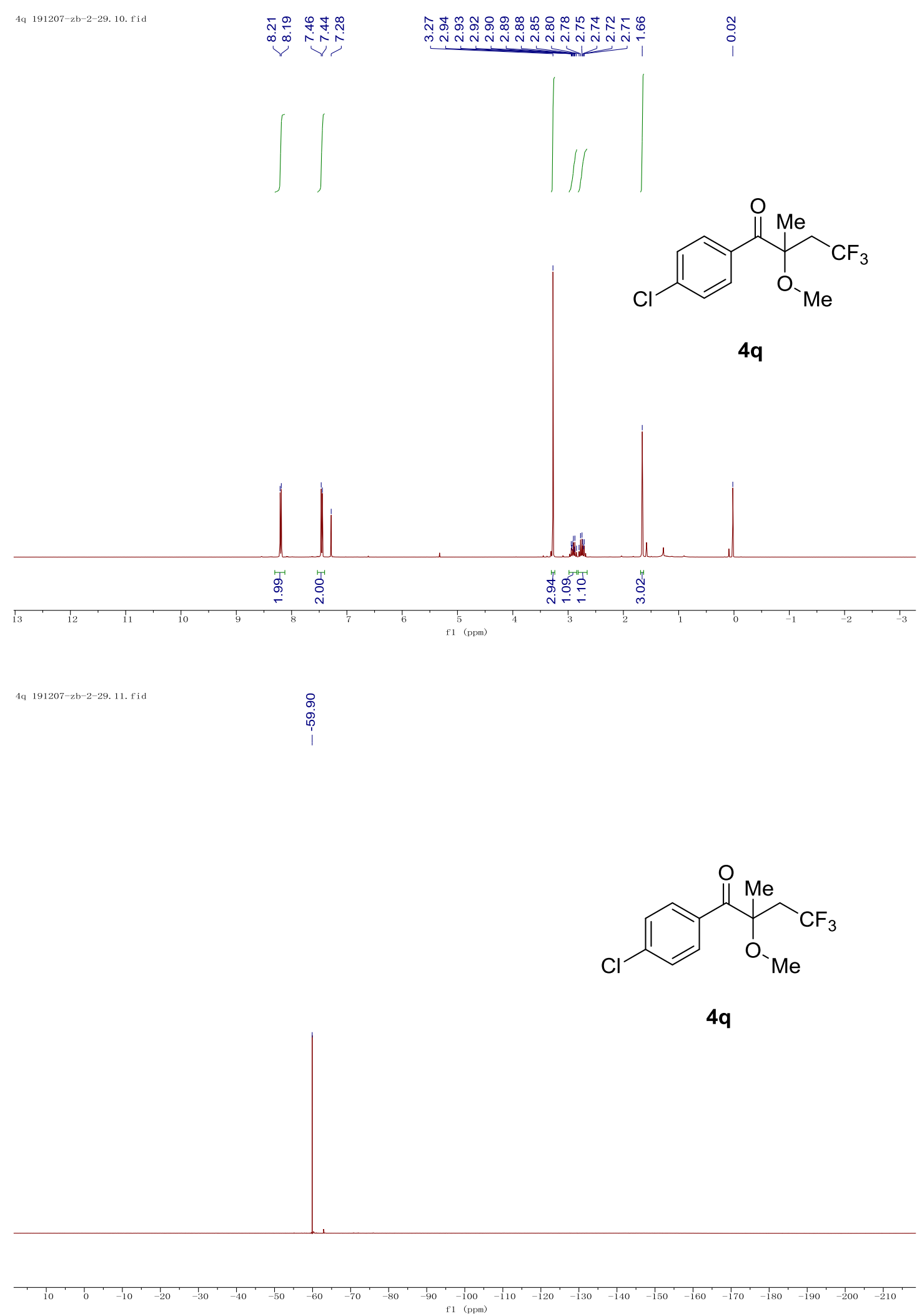

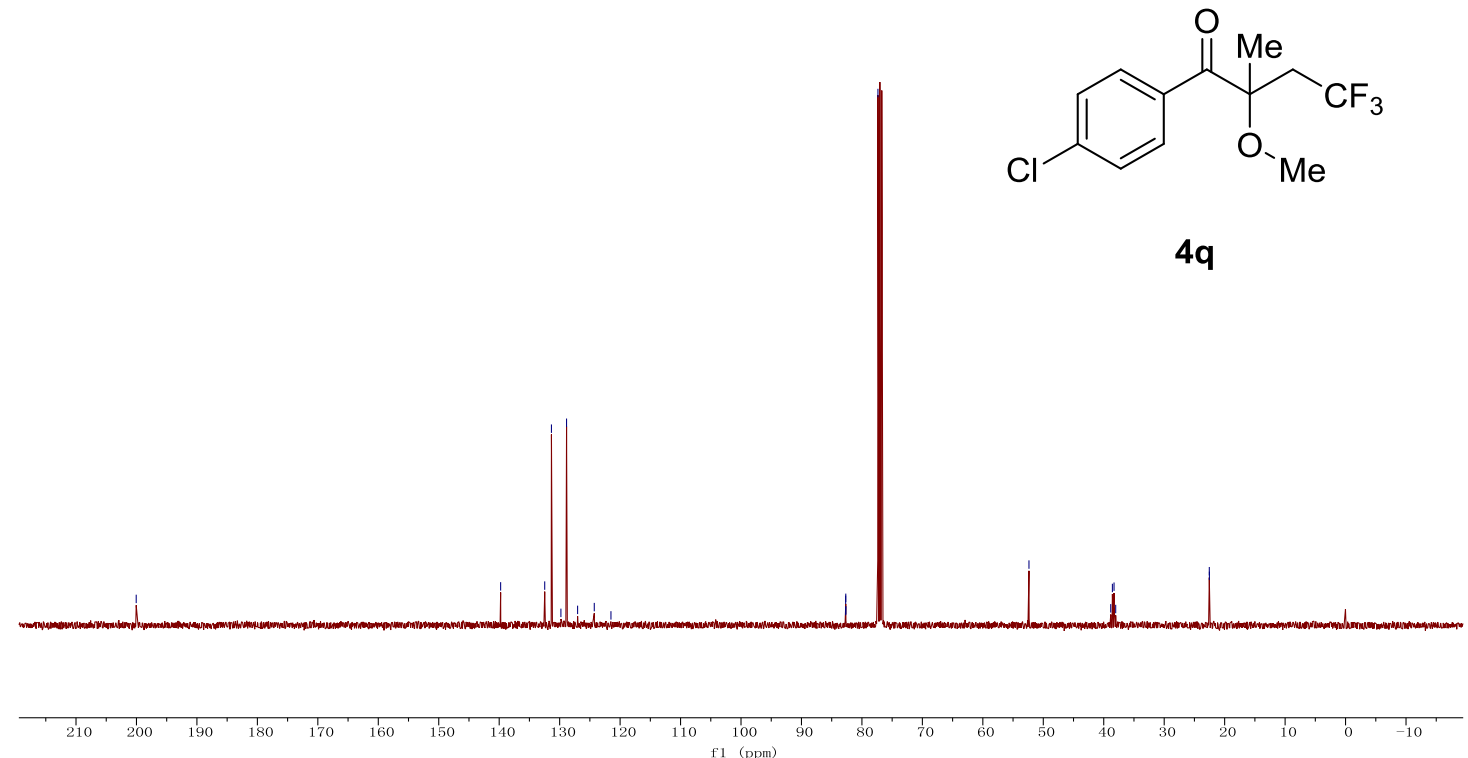

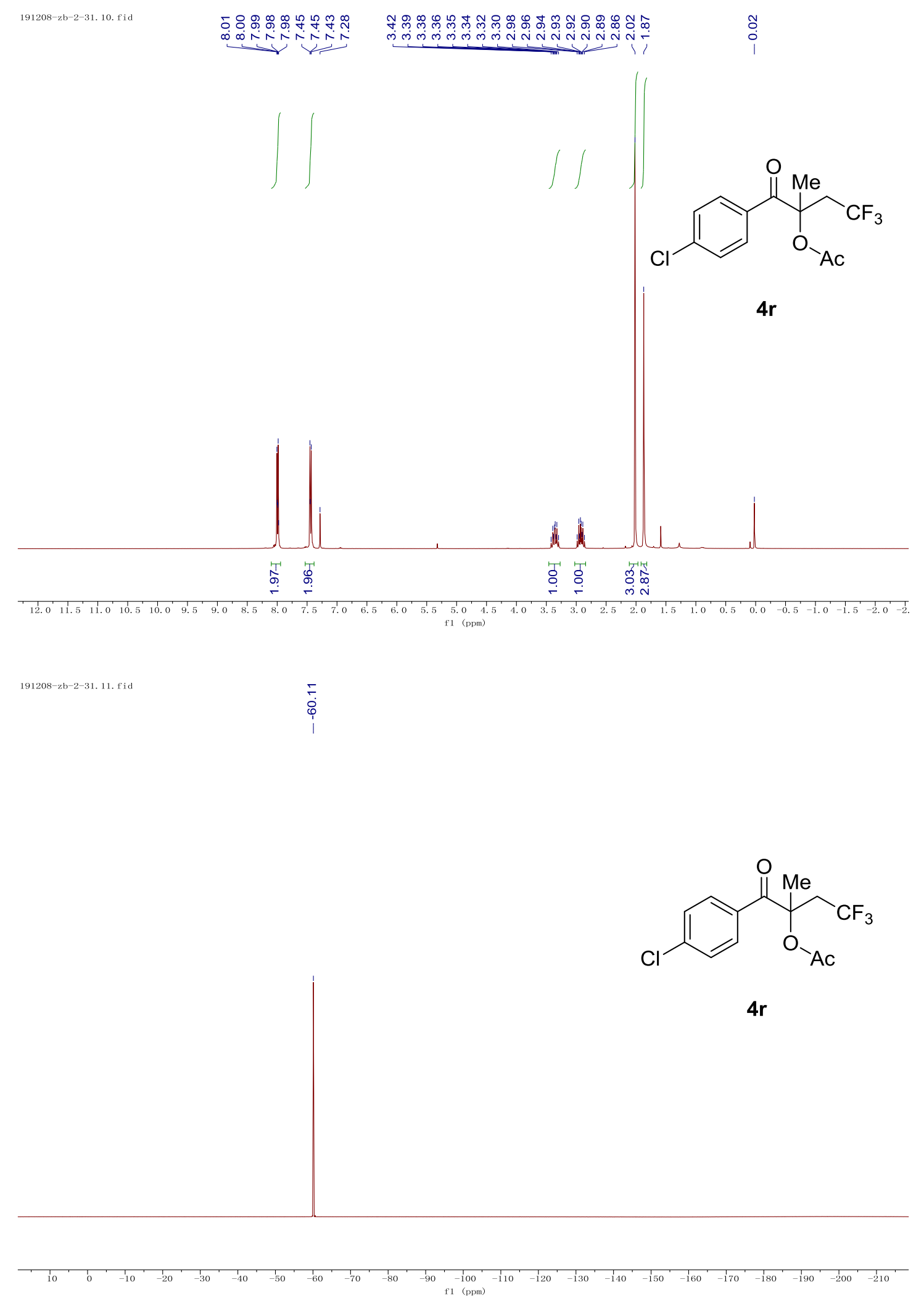


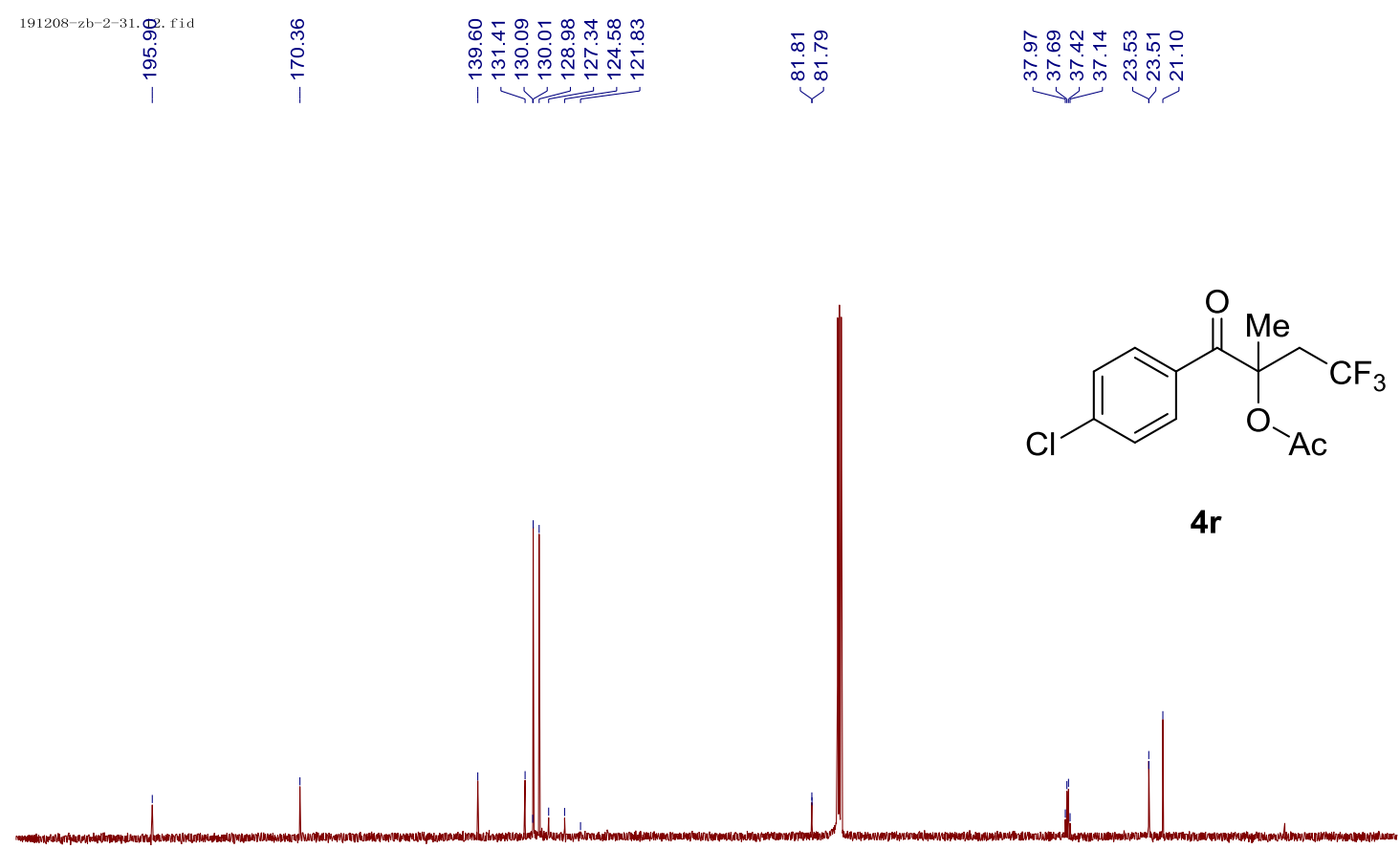




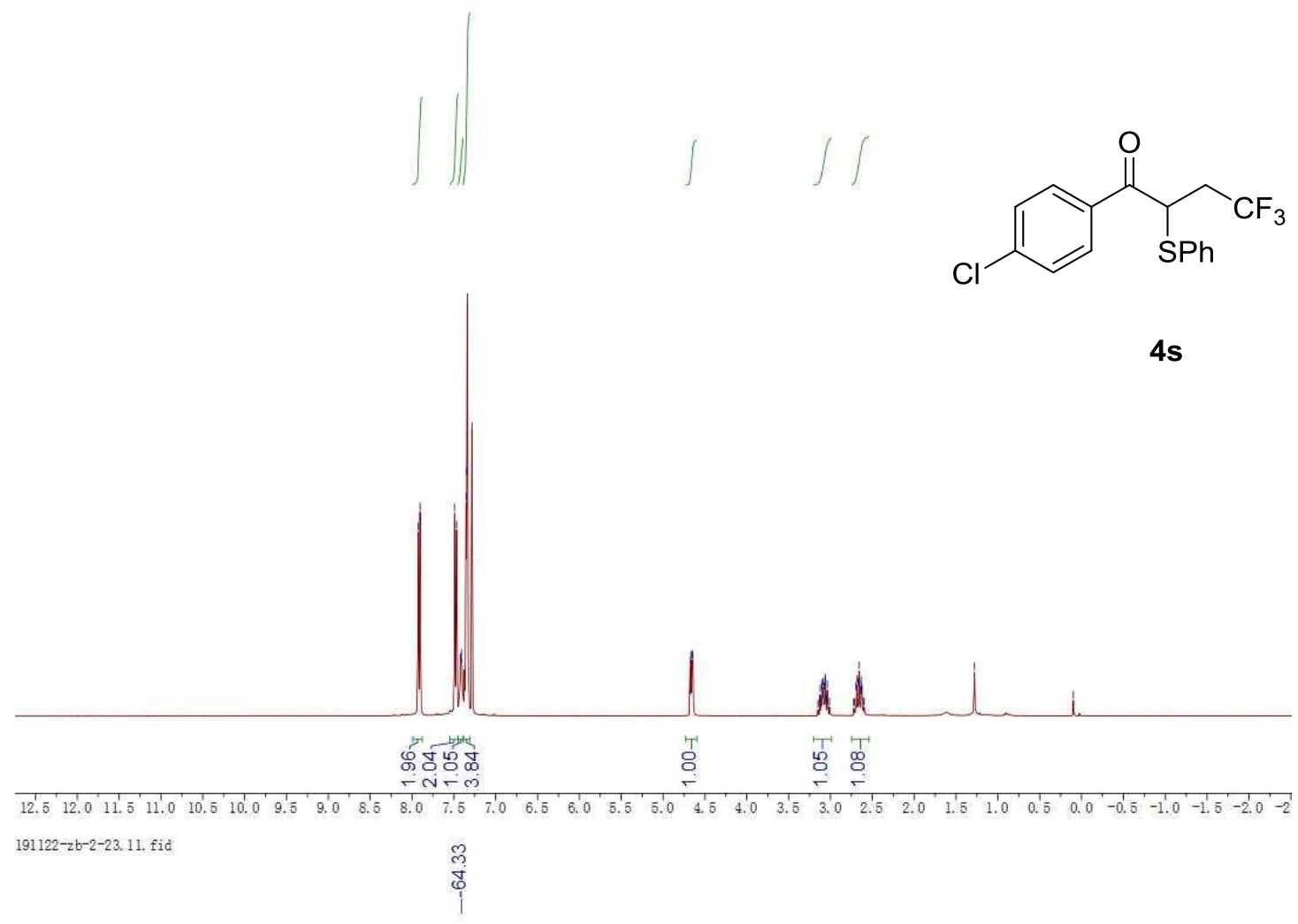<smiles>O=C(c1ccc(Cl)cc1)C(CC(F)(F)F)c1ccccc1</smiles>

4s

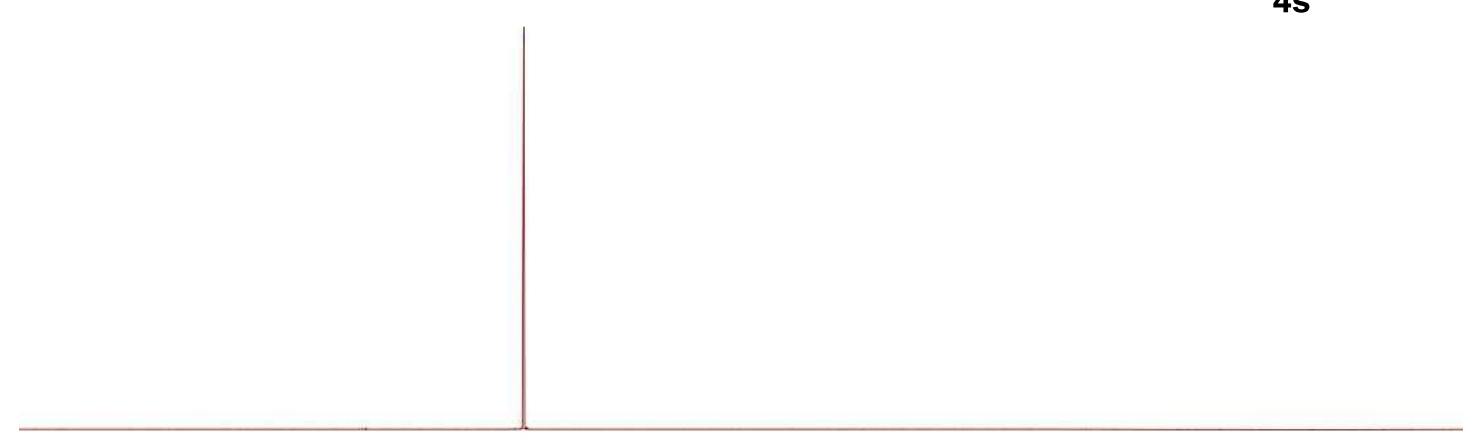




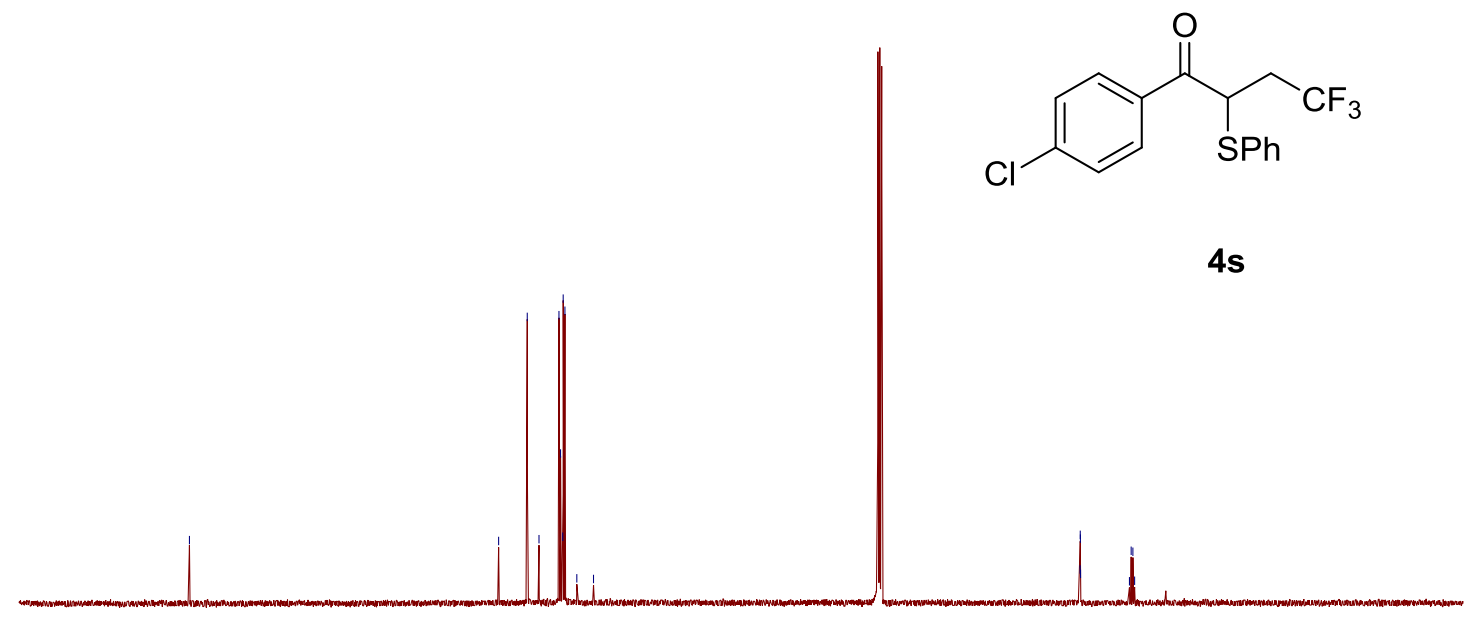



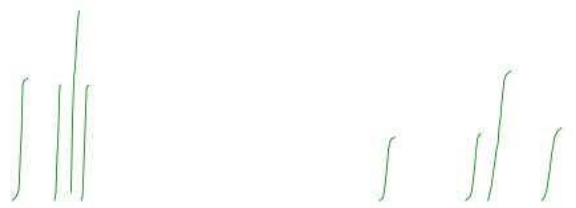<smiles>O=C(c1ccc(Cl)cc1)C(Cc1ccccc1)CC(F)(F)F</smiles>

$4 t$

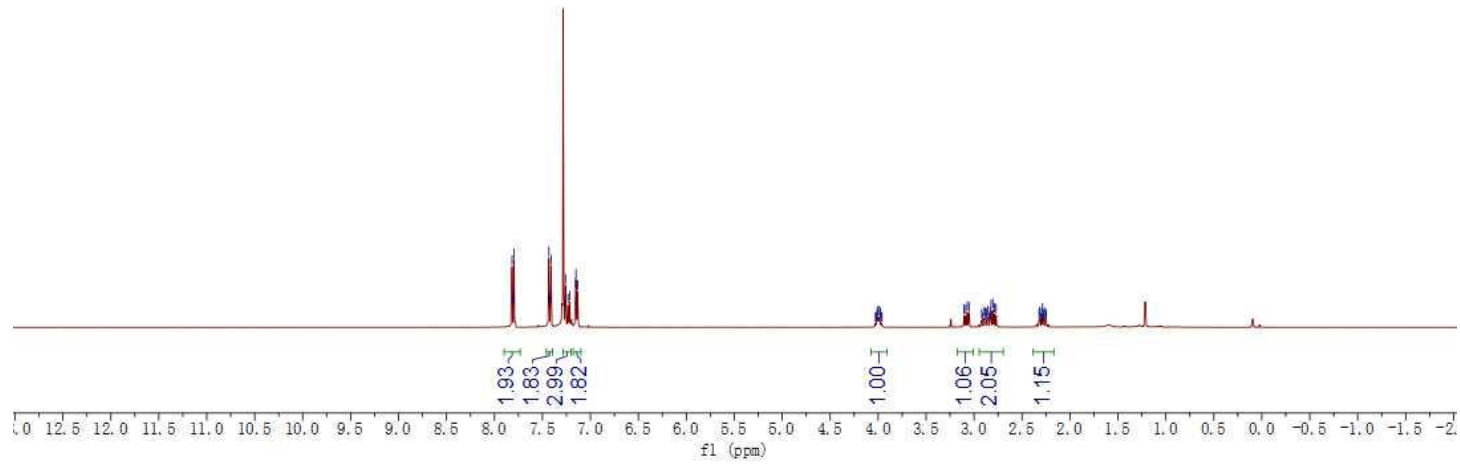
$191121-z b-2-24$
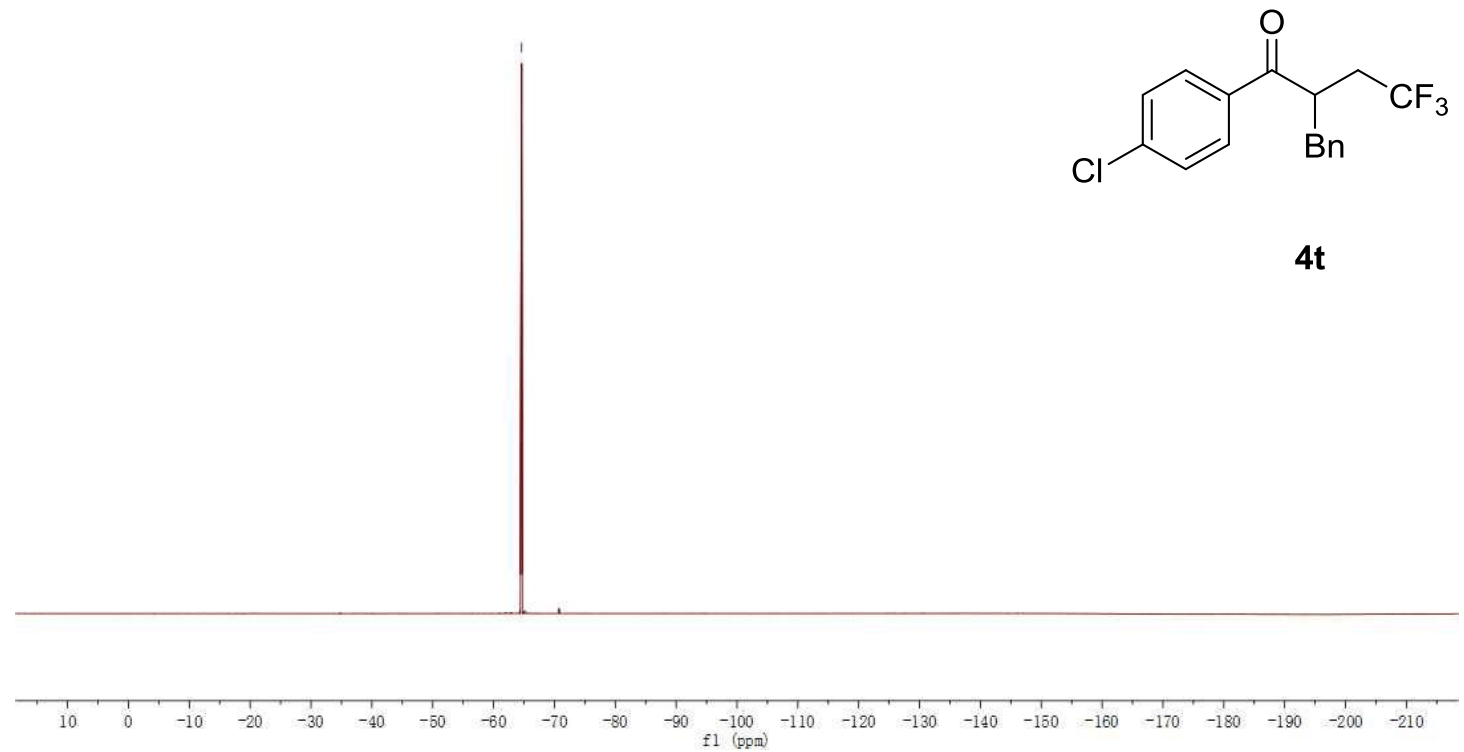


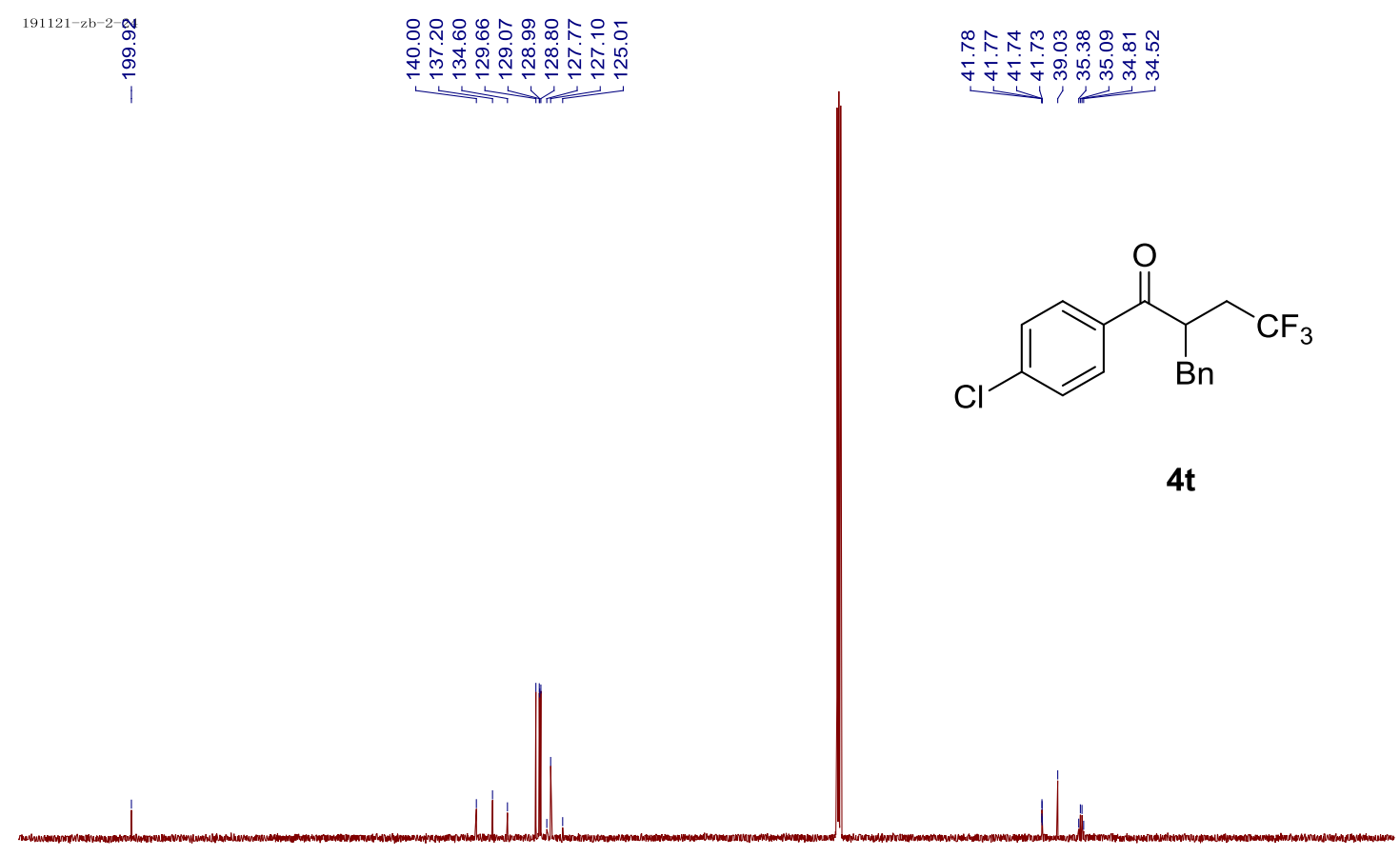




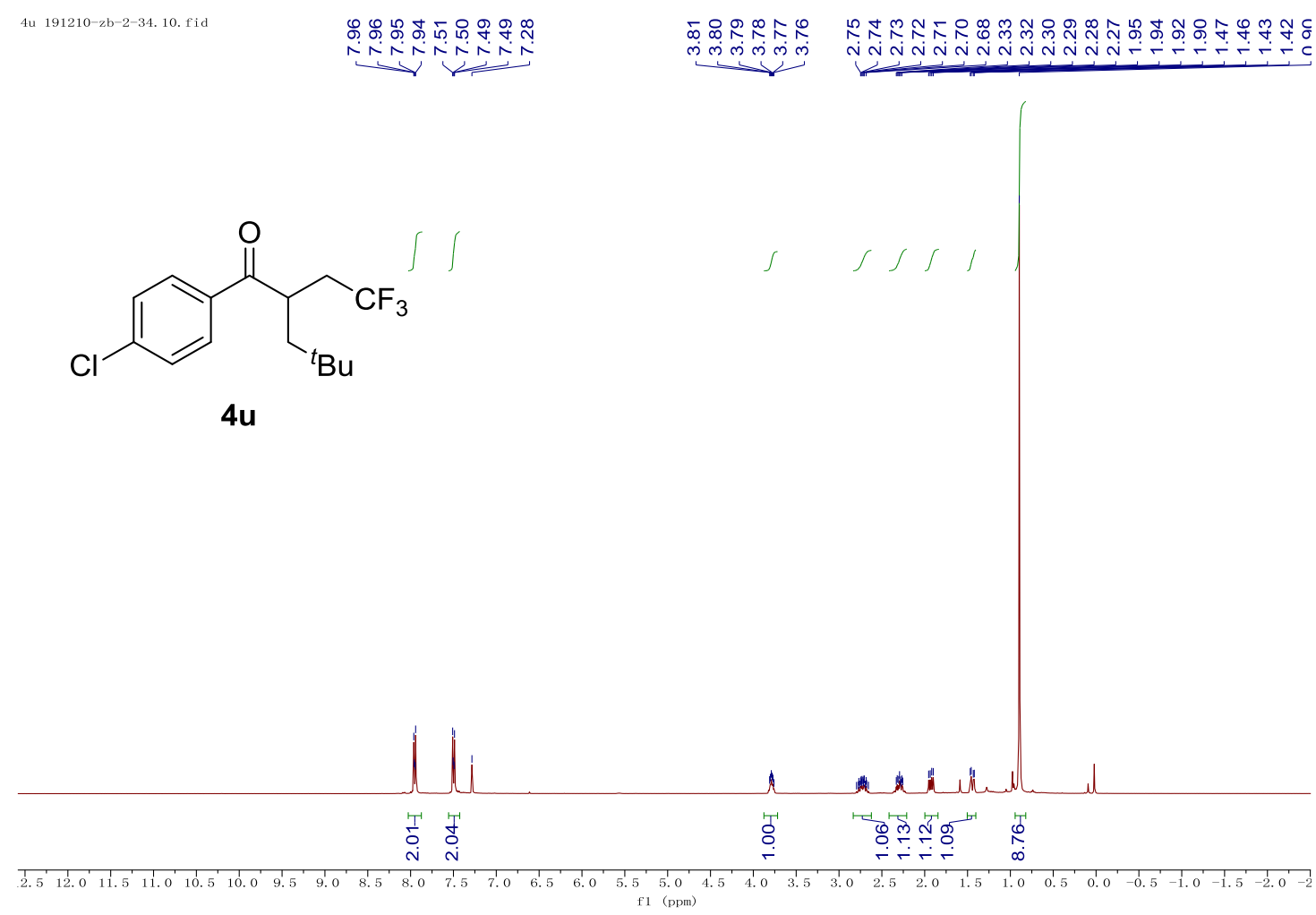

4u 191210-zb-2-34. 11. fid<smiles>CC(C)(C)CC(CC(F)(F)F)C(=O)c1ccc(Cl)cc1</smiles>

$4 u$

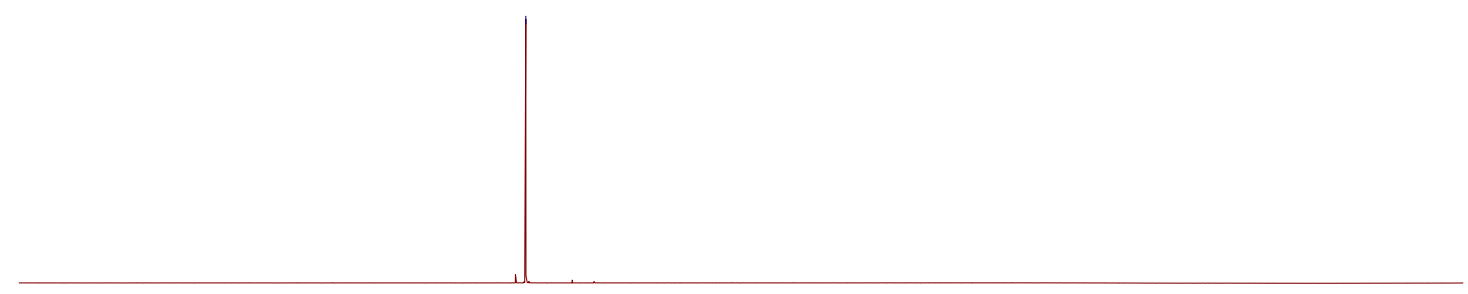


4u $191210-$ zlm2-34. 12. fid ڤั่

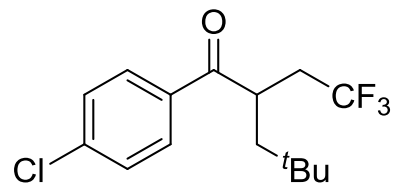

$4 u$

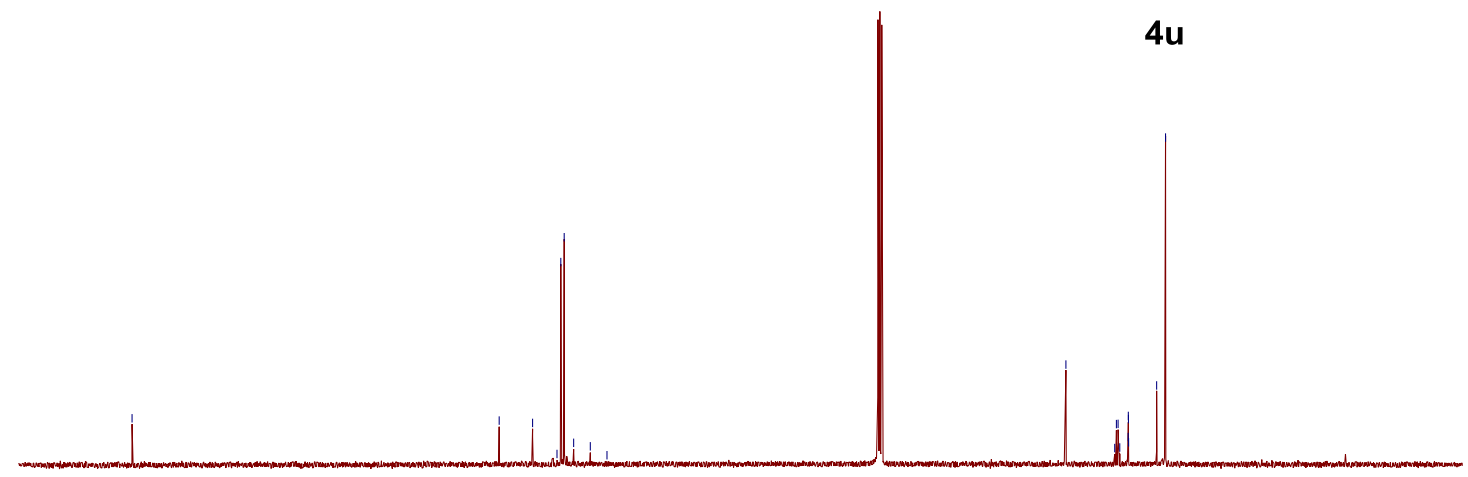

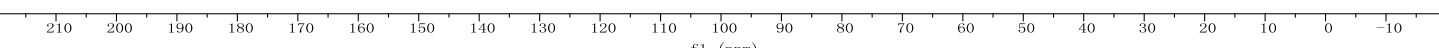



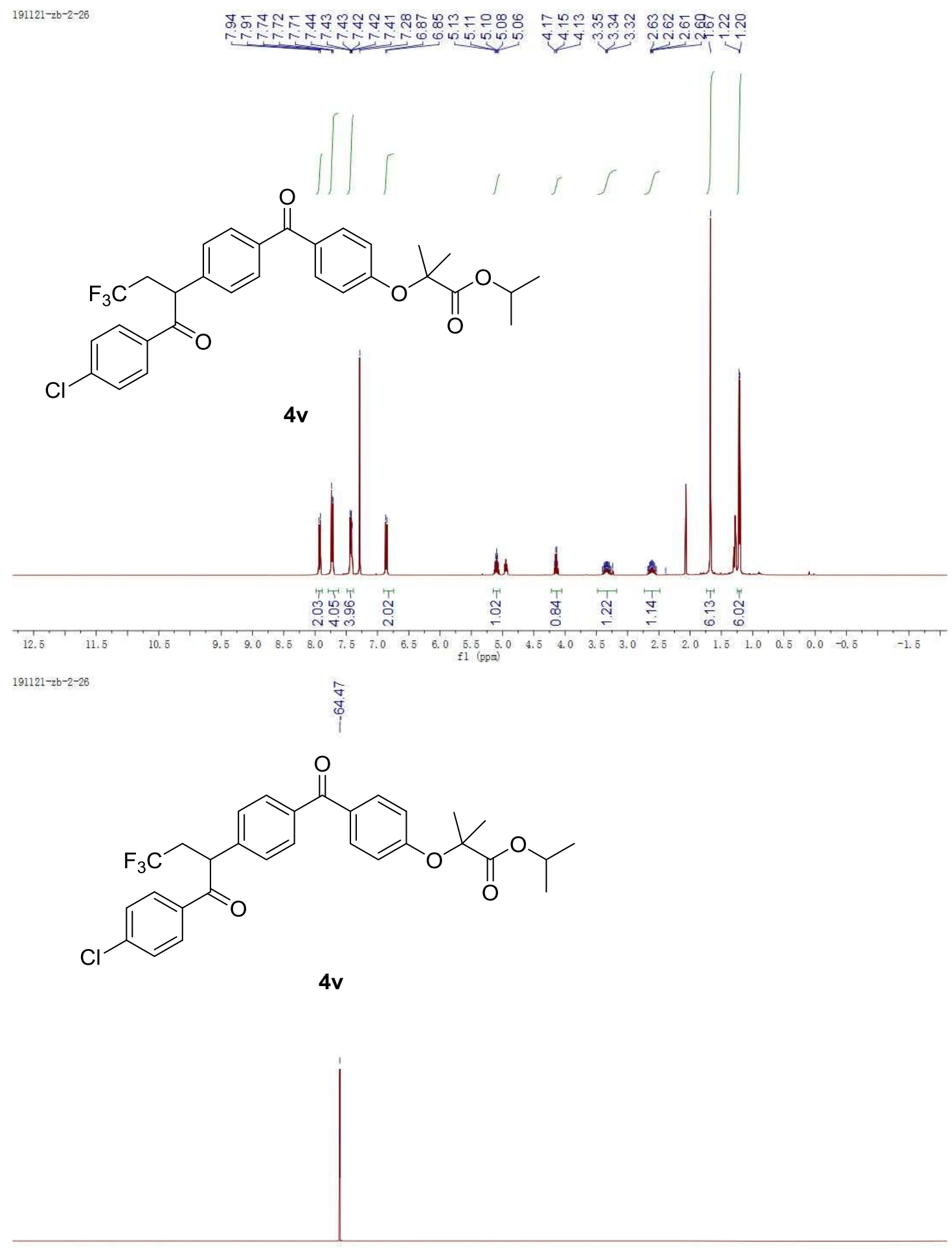


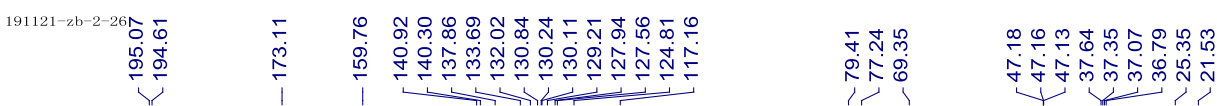
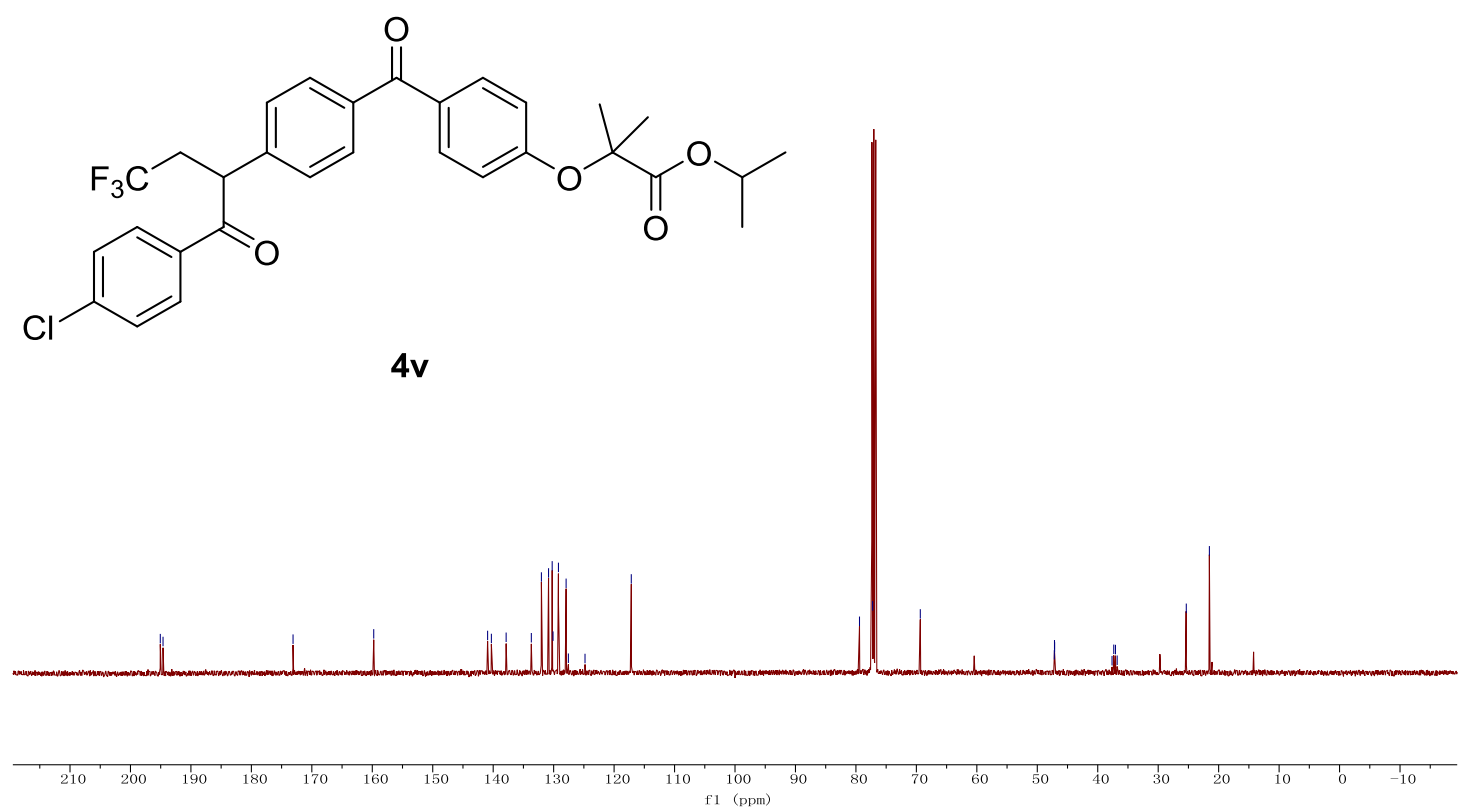


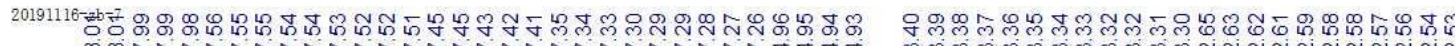

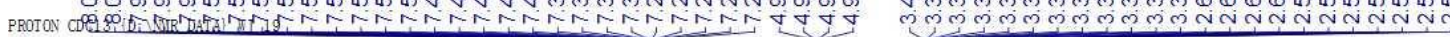

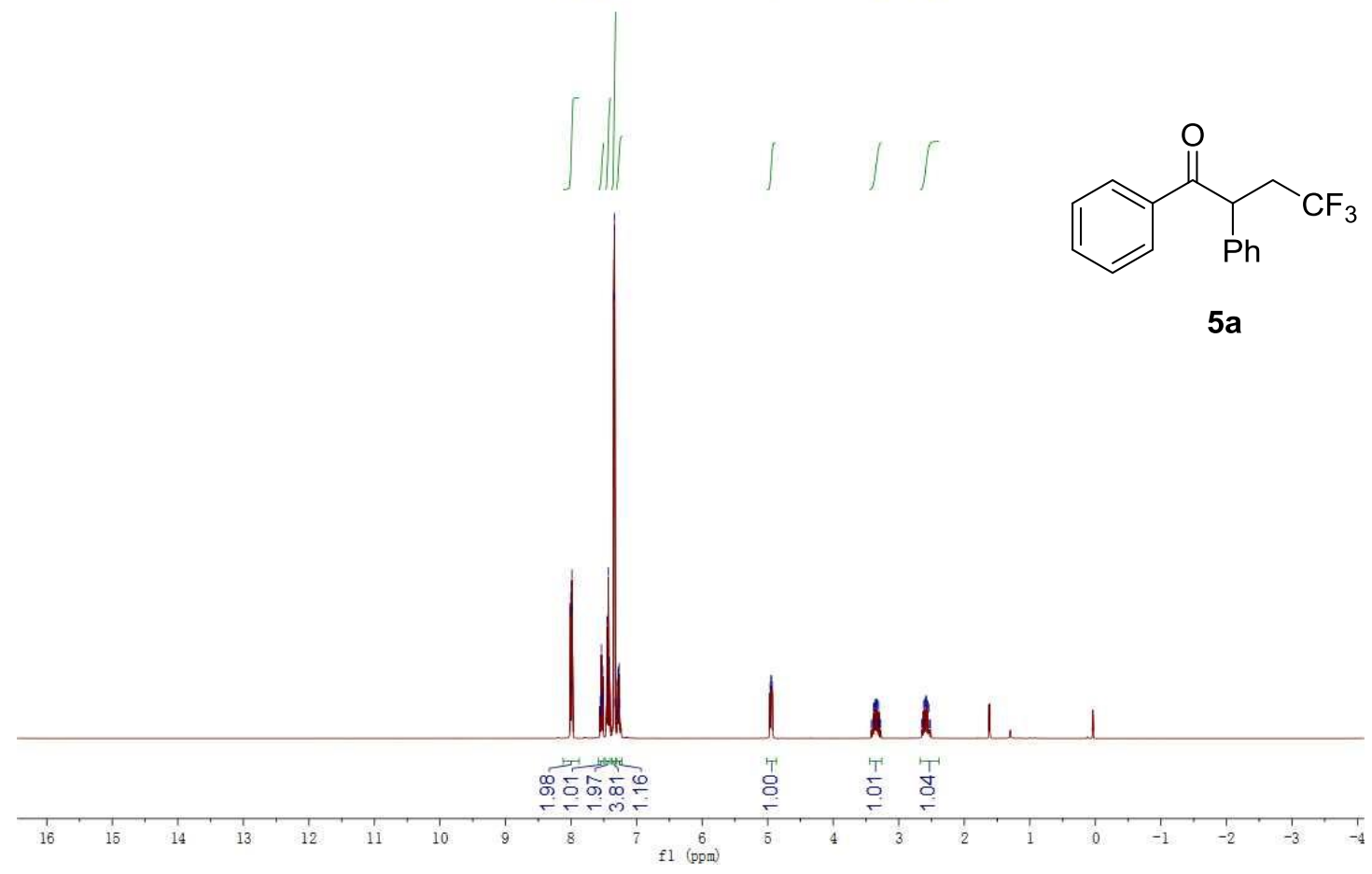

$20191116-z b-7$

F19CPD CDC13 \{D: LWIR_DATA\} WJ 19

@
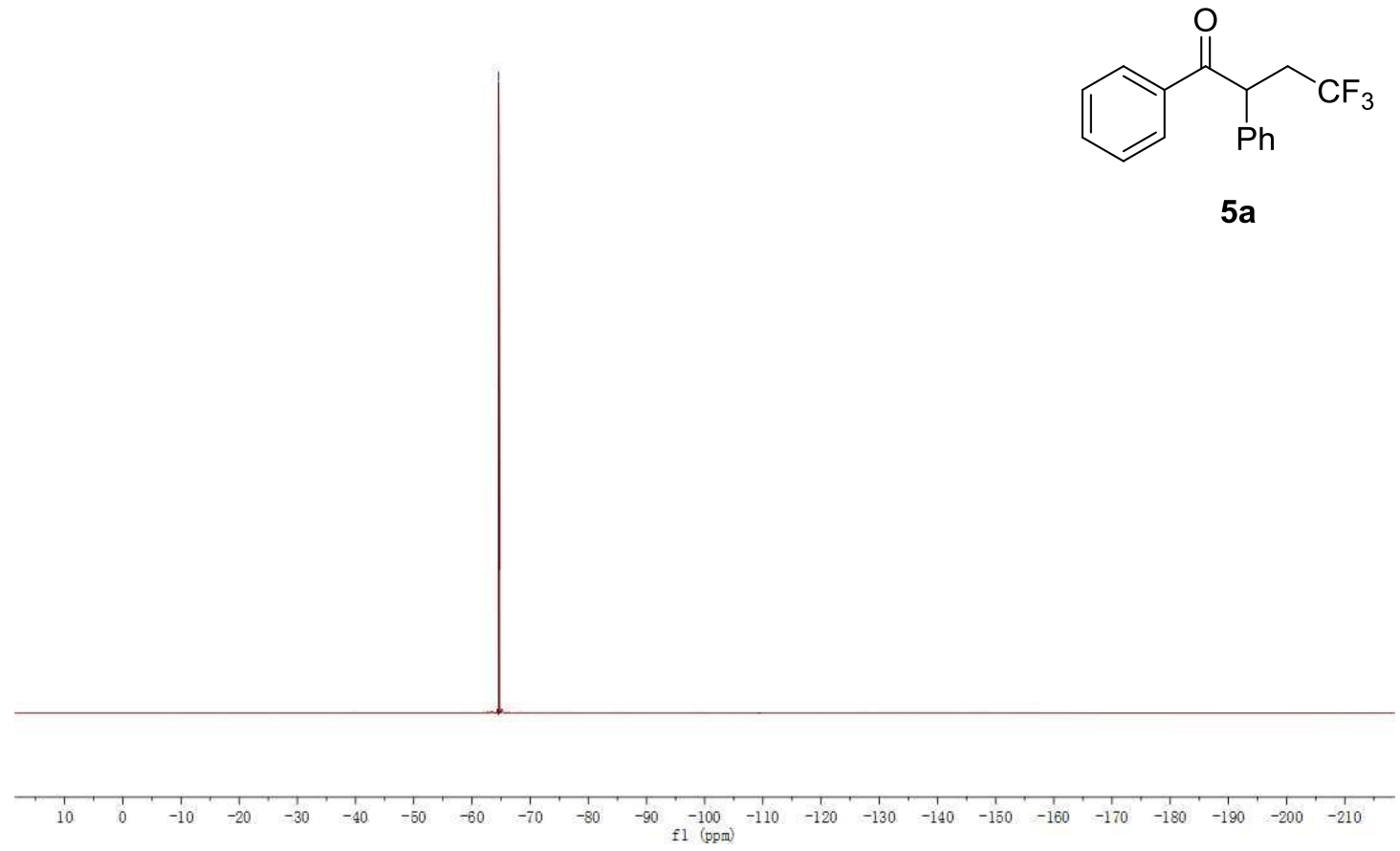


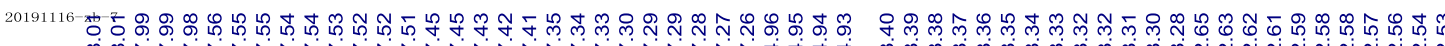

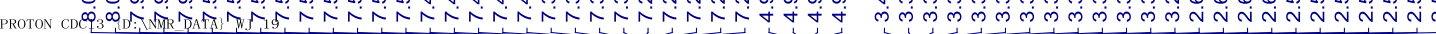

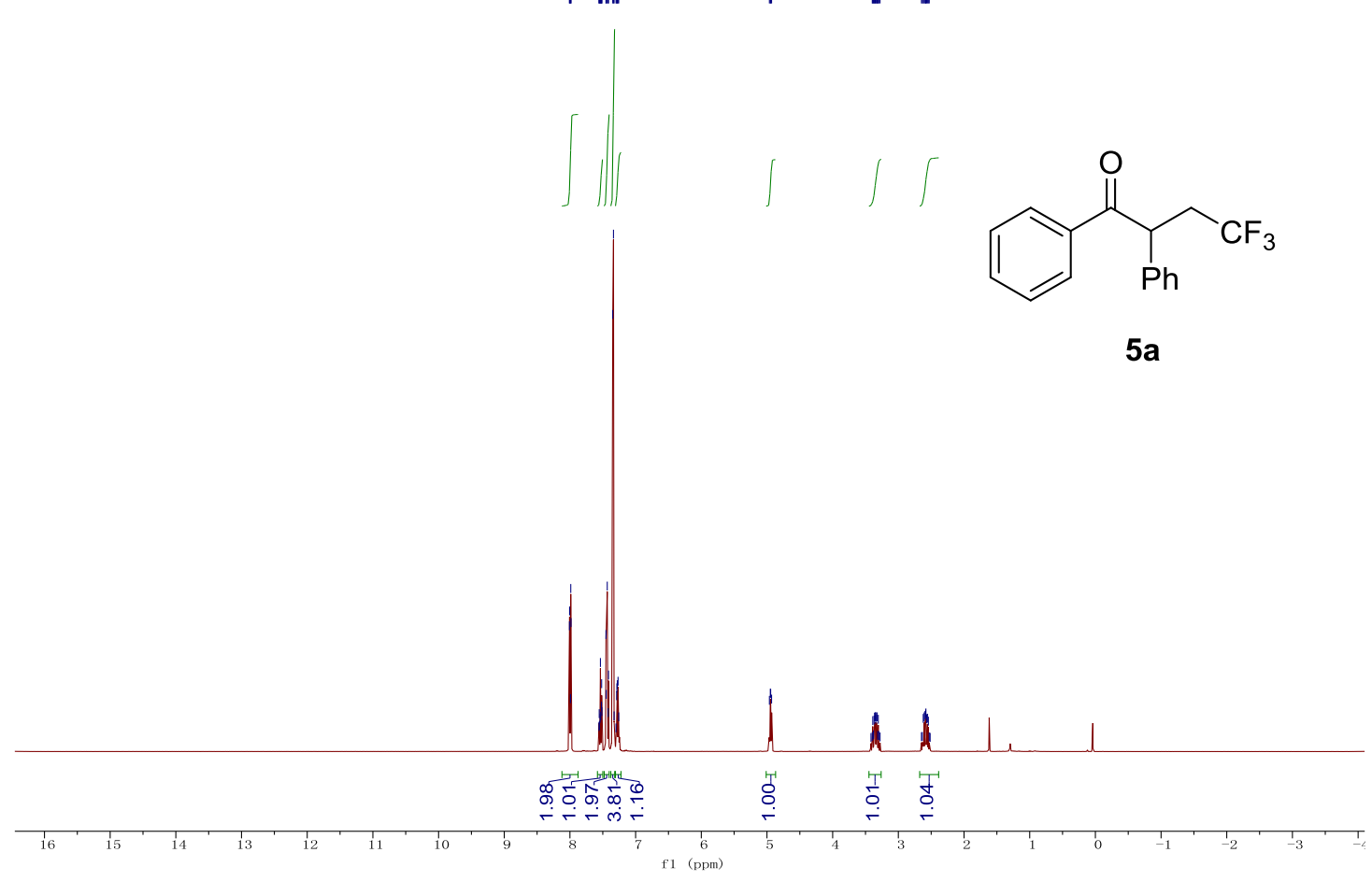




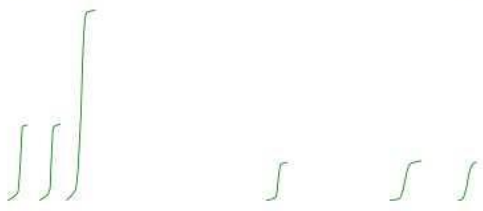<smiles>O=C(c1ccc(C(F)(F)F)cc1)C(CC(F)(F)F)c1ccccc1</smiles>

$5 b$

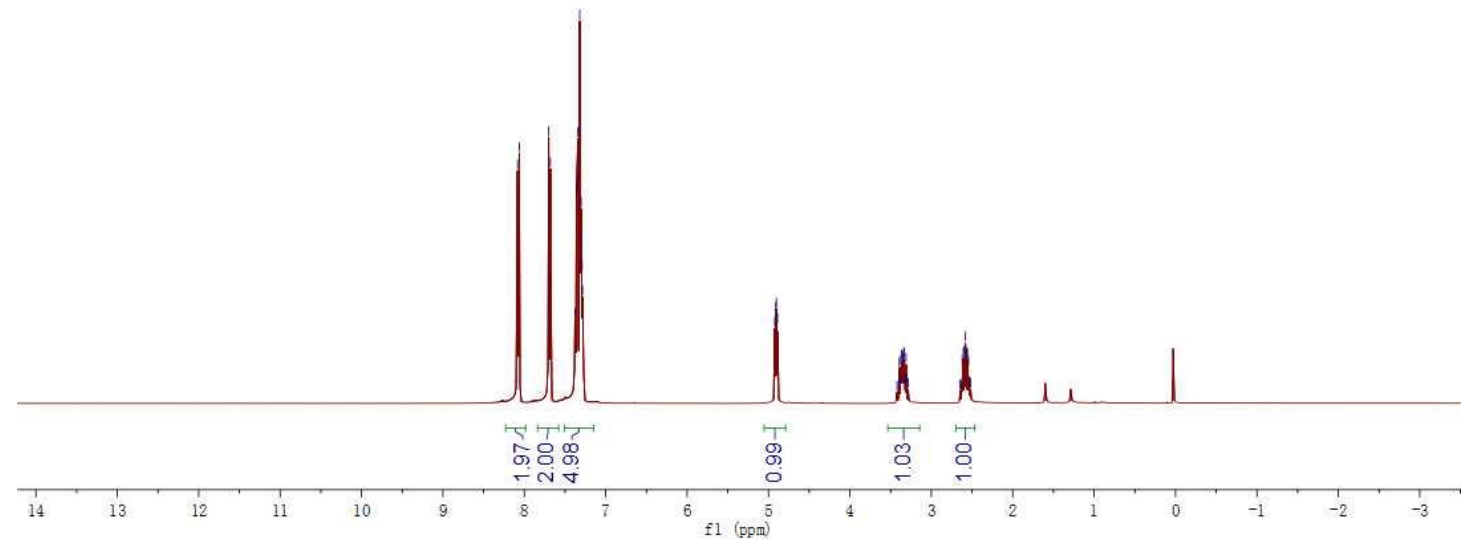

$191114-z b-3$

$\stackrel{\infty}{\sim} \mathbb{8}$

i<smiles>O=C(c1ccc(C(F)(F)F)cc1)C(CC(F)(F)F)c1ccccc1</smiles>

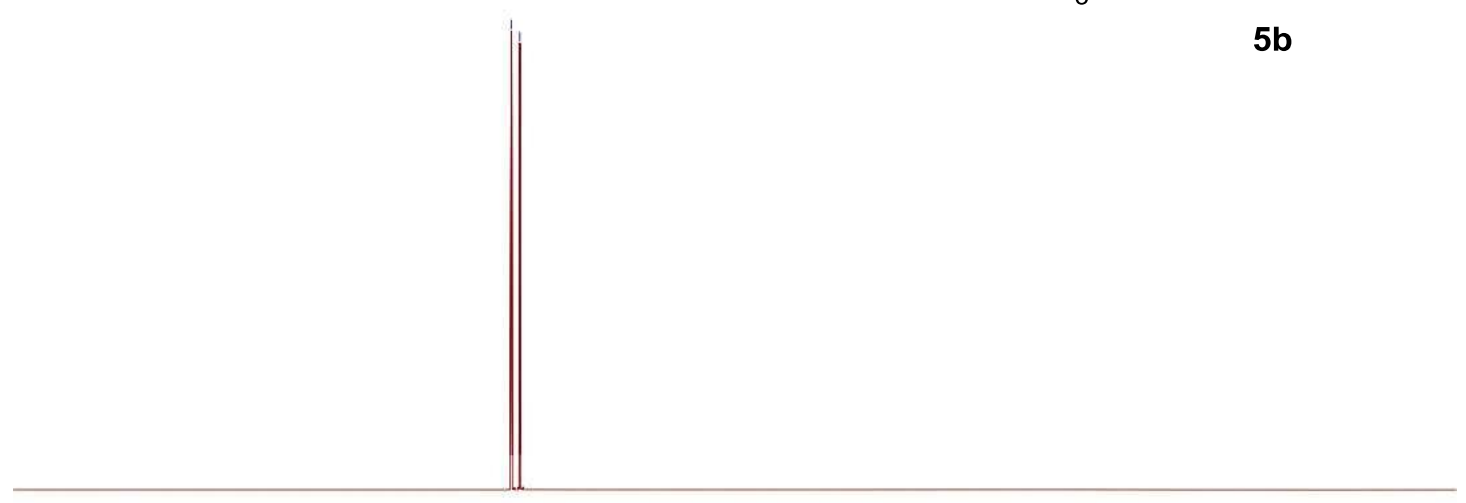

1
10 
<smiles>O=C(c1ccc(C(F)(F)F)cc1)C(CC(F)(F)F)c1ccccc1</smiles>

$5 b$

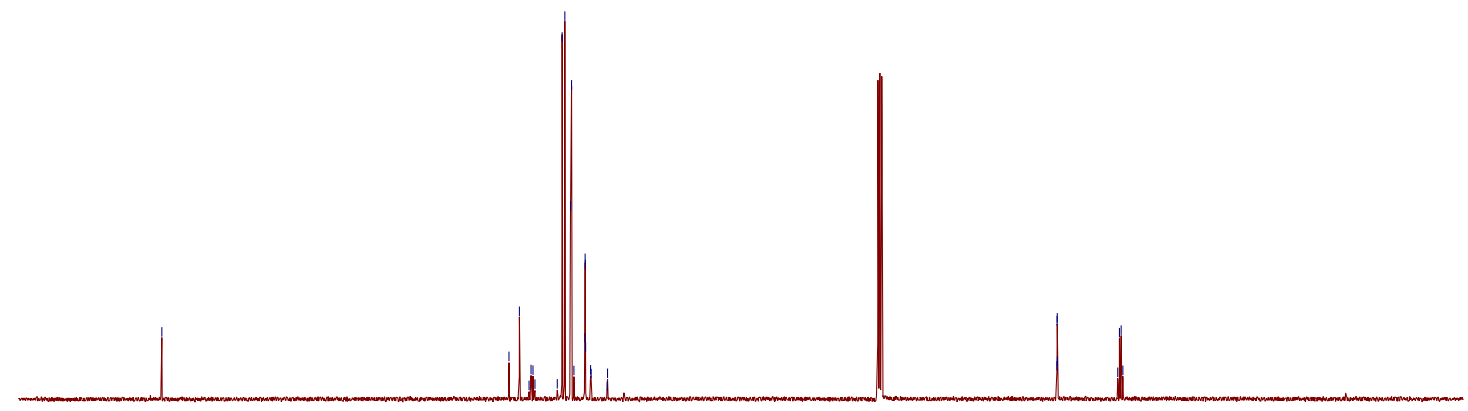

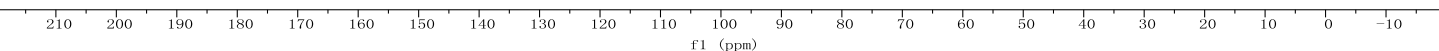




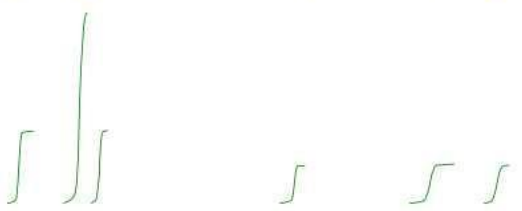<smiles>O=C(c1ccc(F)cc1)C(CC(F)(F)F)c1ccccc1</smiles>

$5 c$
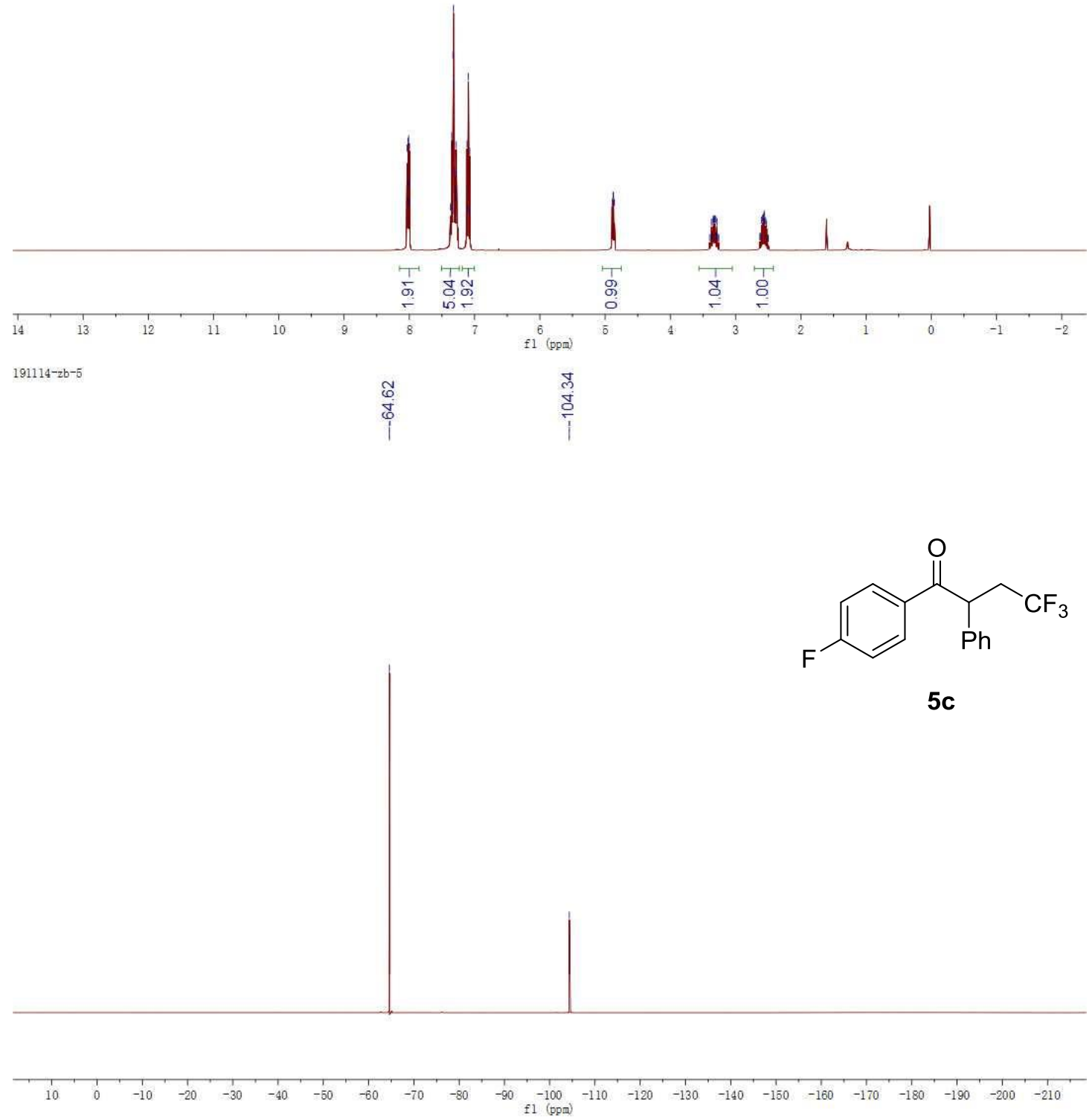


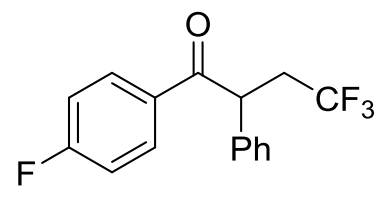

$5 c$

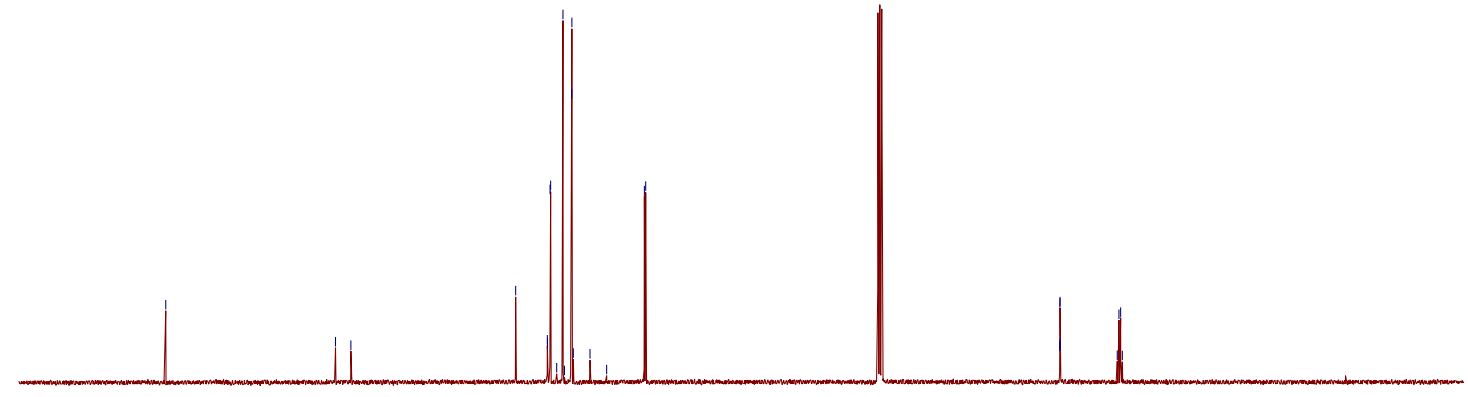

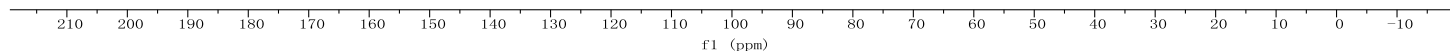




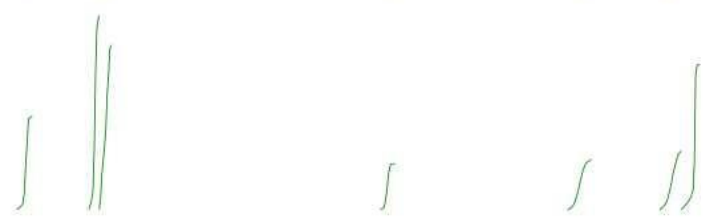<smiles>Cc1ccc(C(=O)C(CC(F)(F)F)c2ccccc2)cc1</smiles>

$5 d$
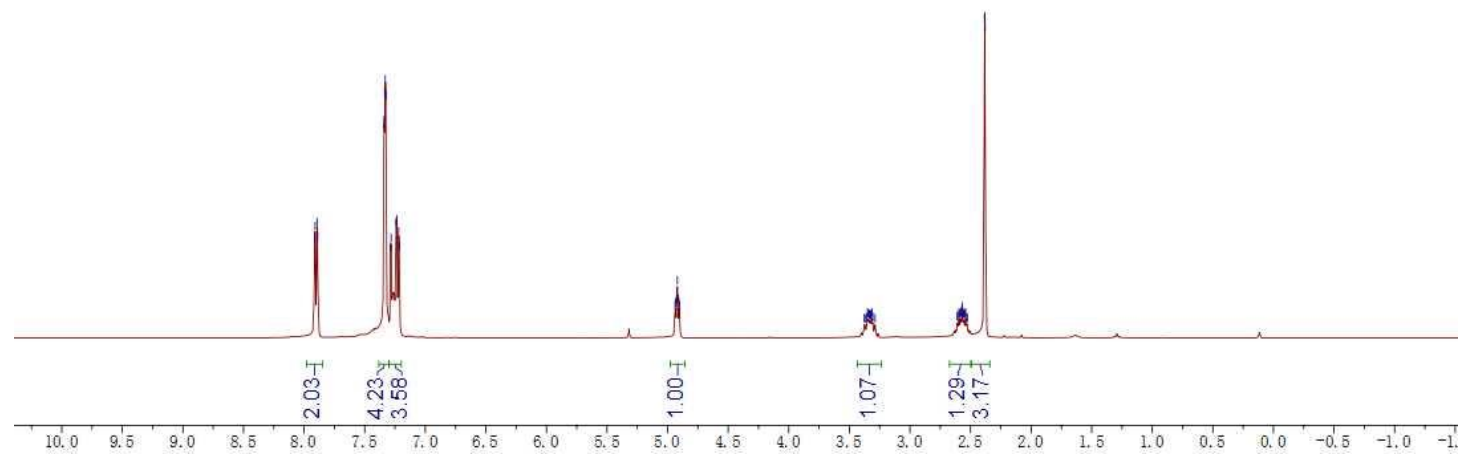

$191121-z b-1-1,21$. fid

@<smiles>Cc1ccc(C(=O)C(CC(F)(F)F)c2ccccc2)cc1</smiles>

$5 d$

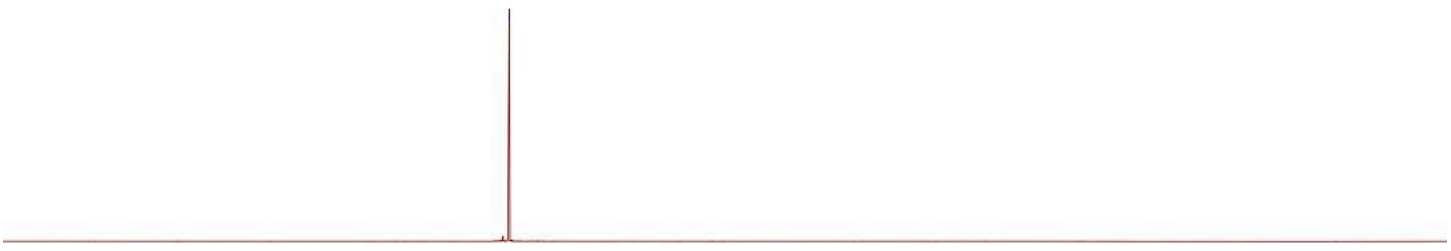




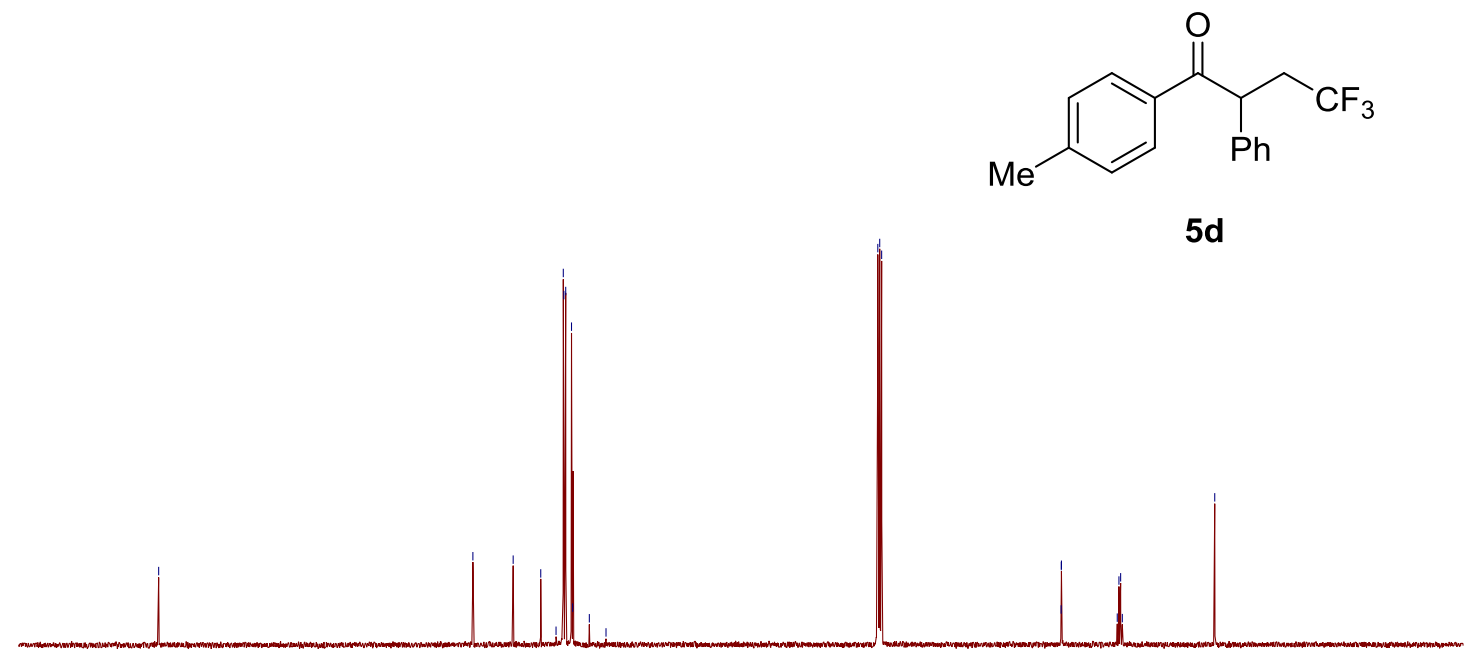



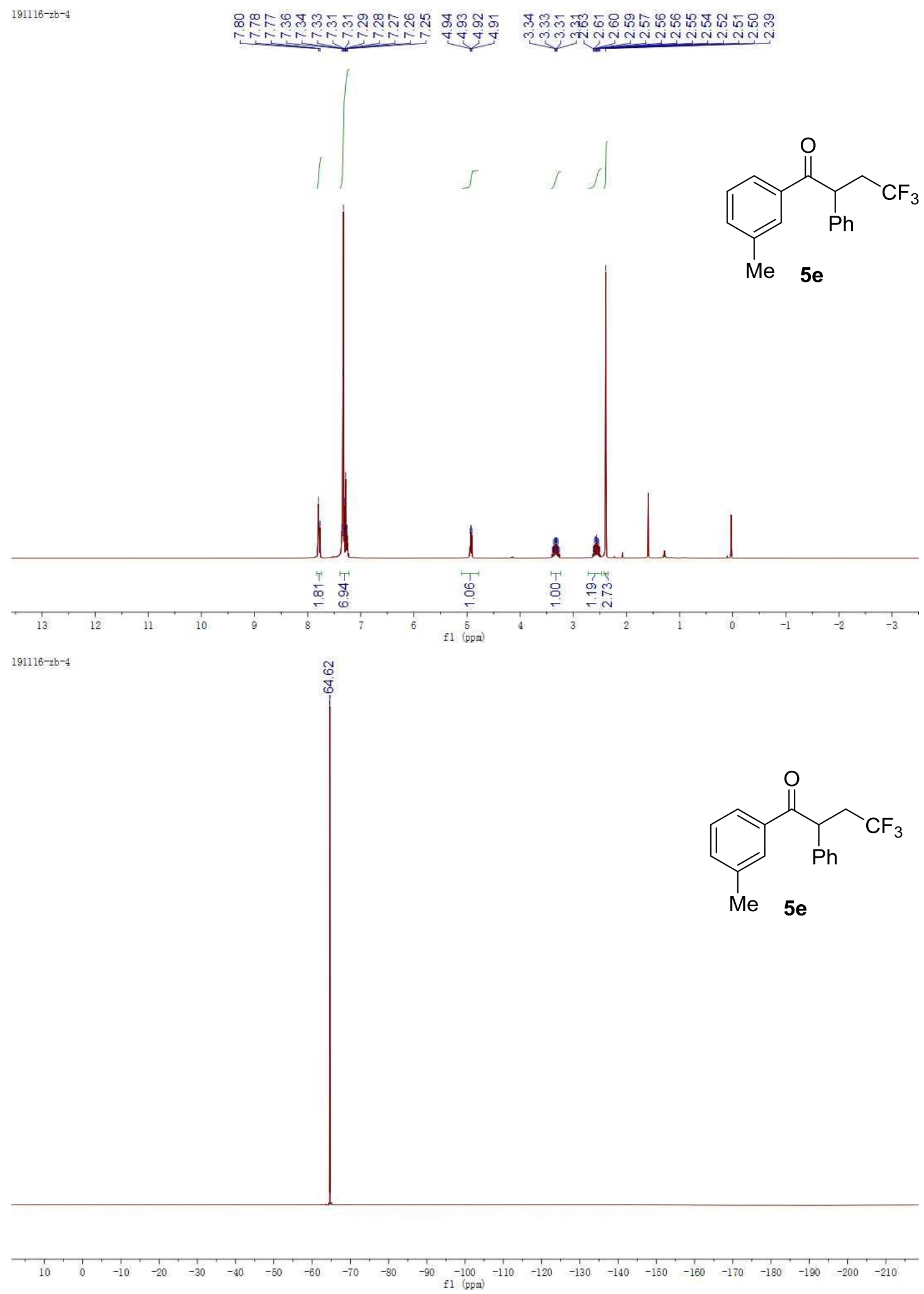


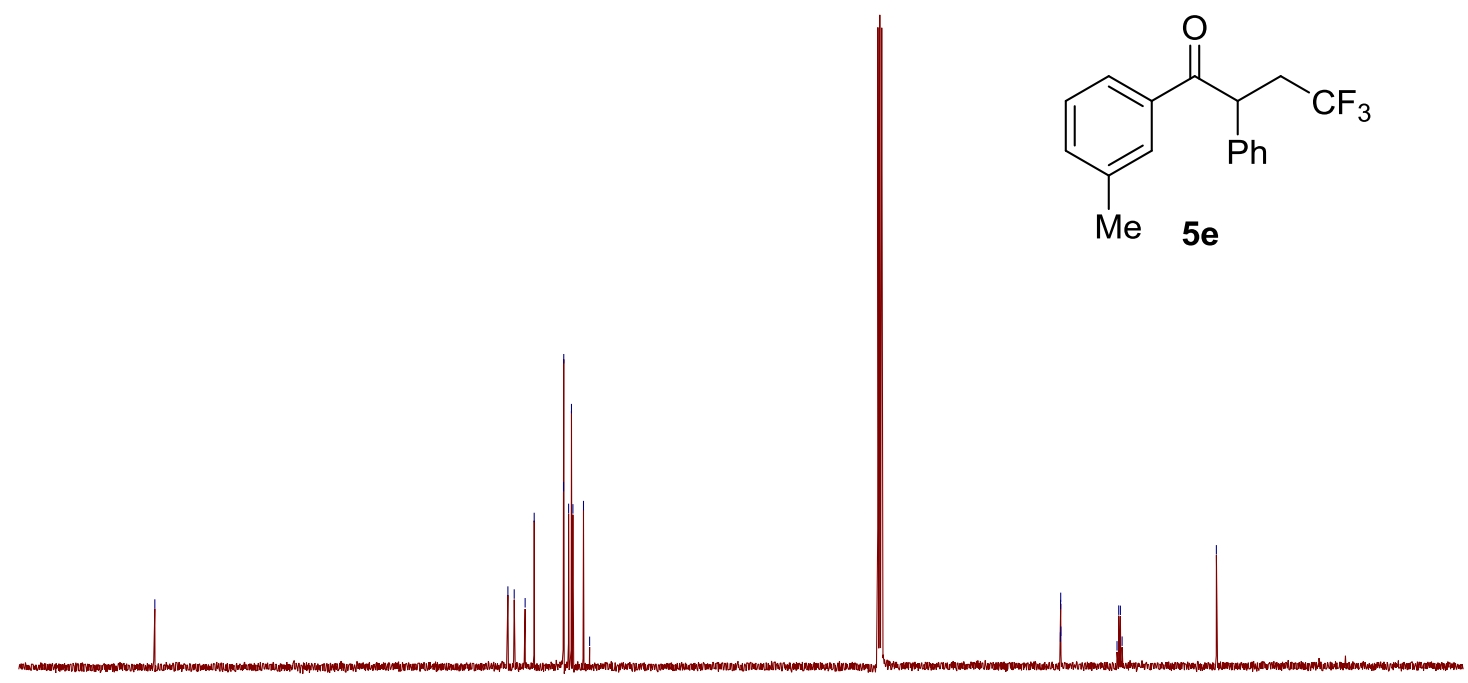




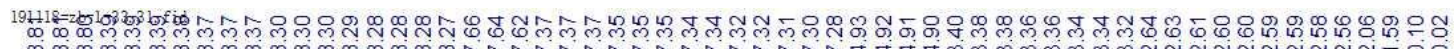
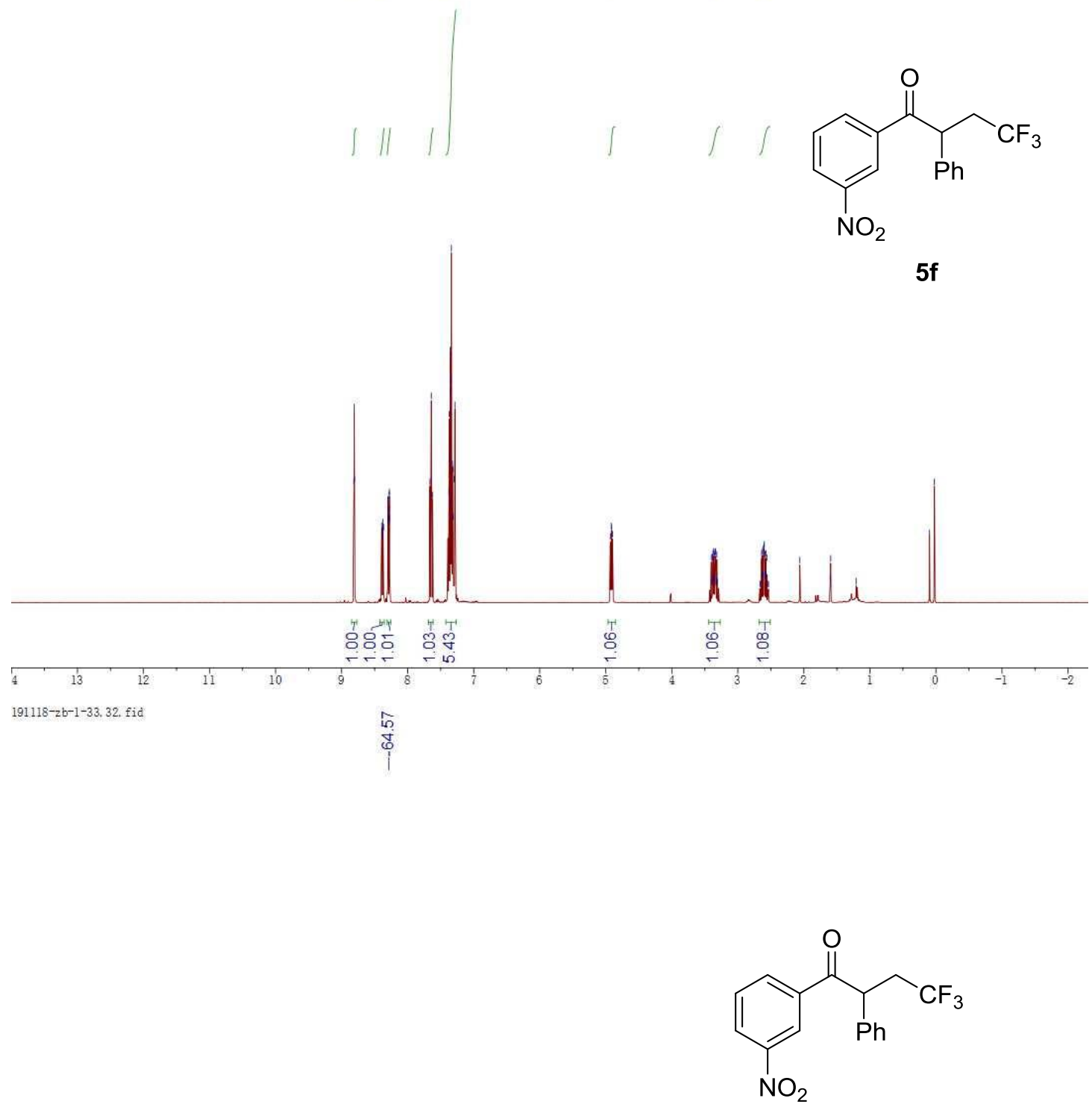

$5 f$

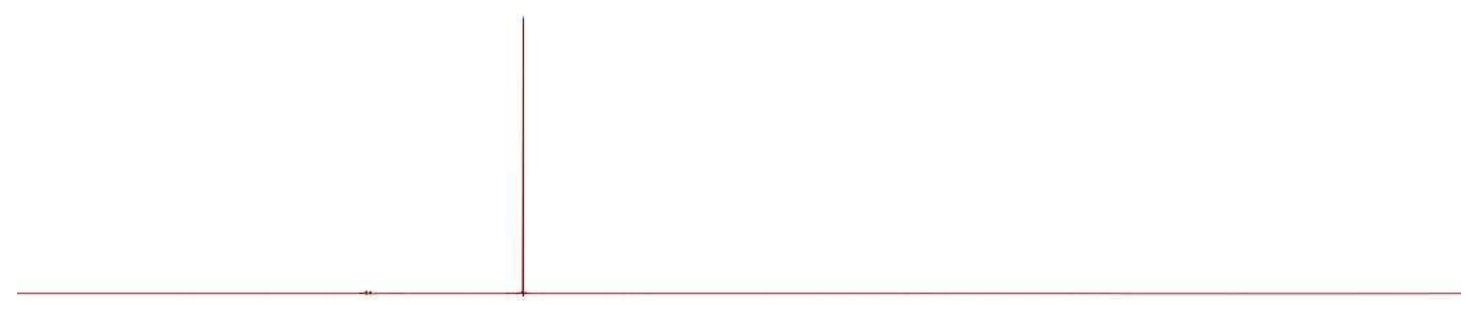



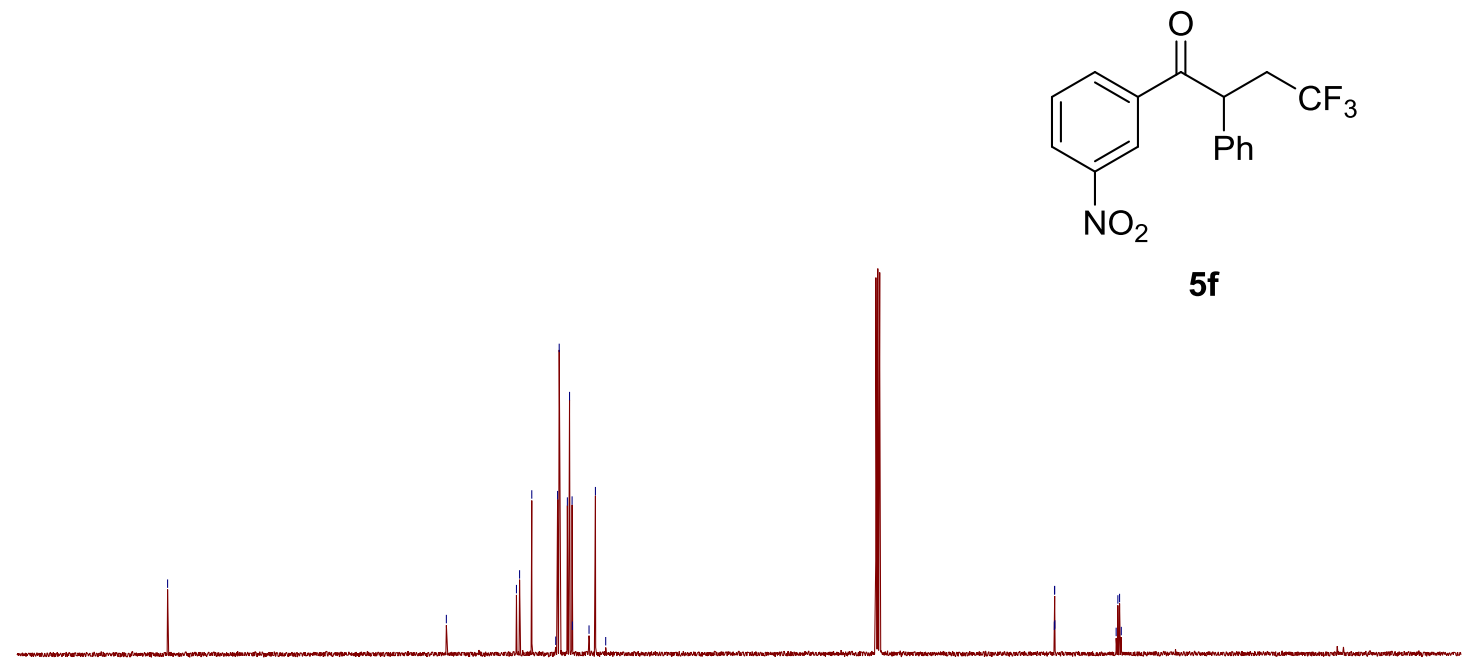


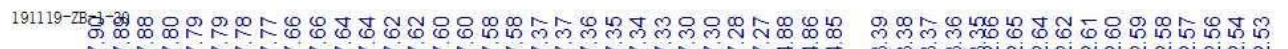

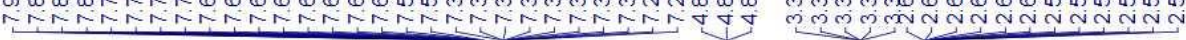
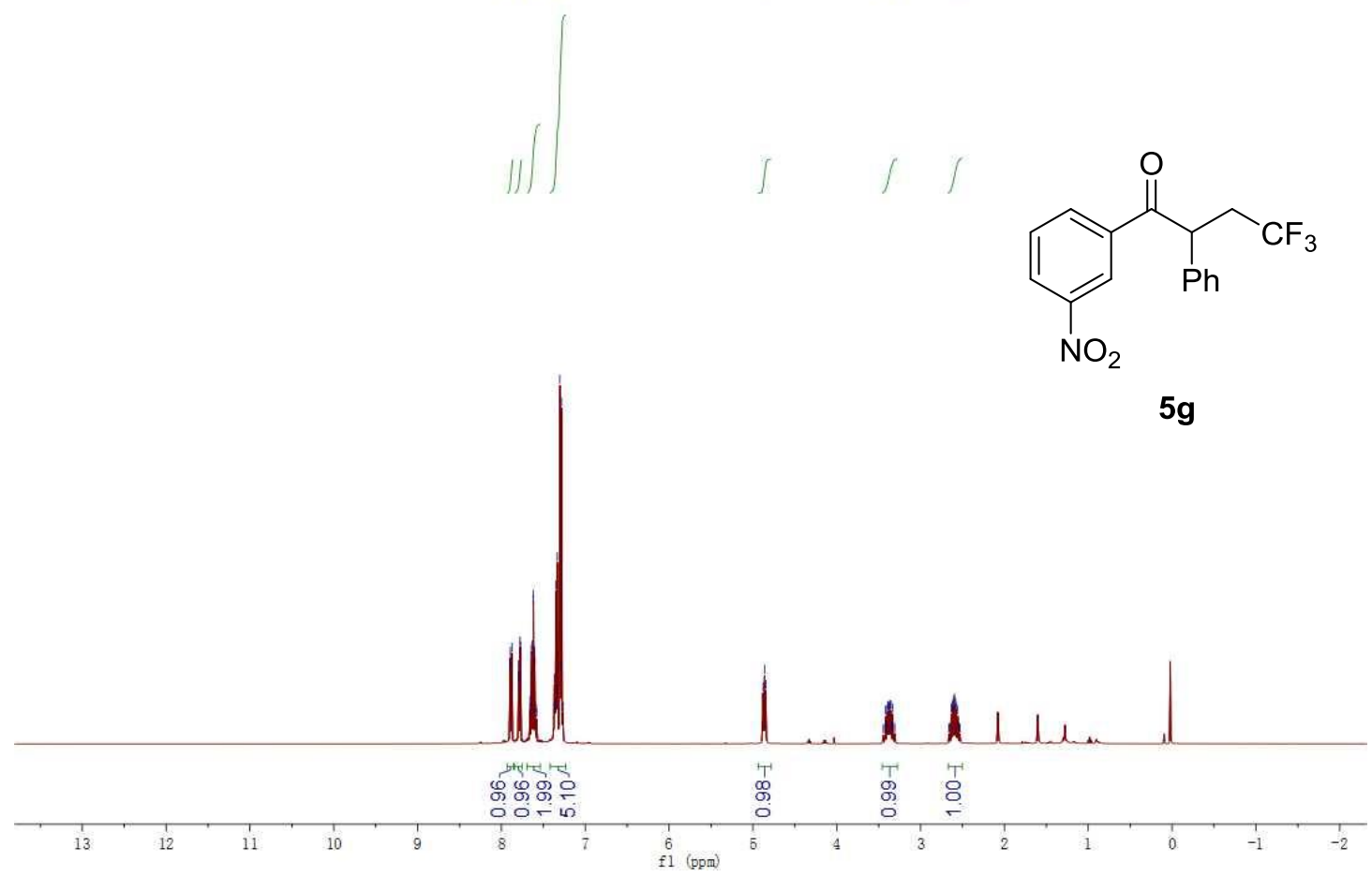

$191119-2 B-1-30$

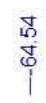
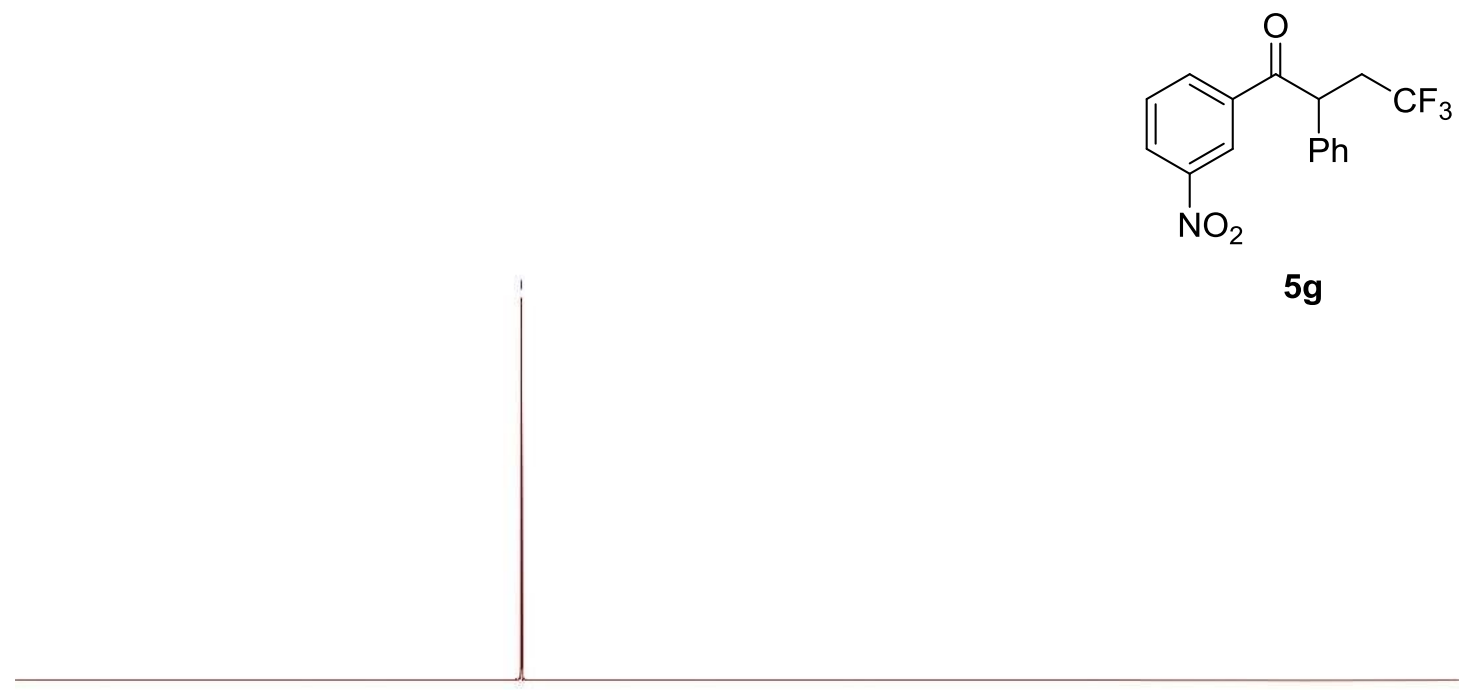

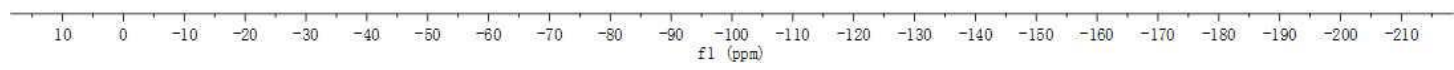




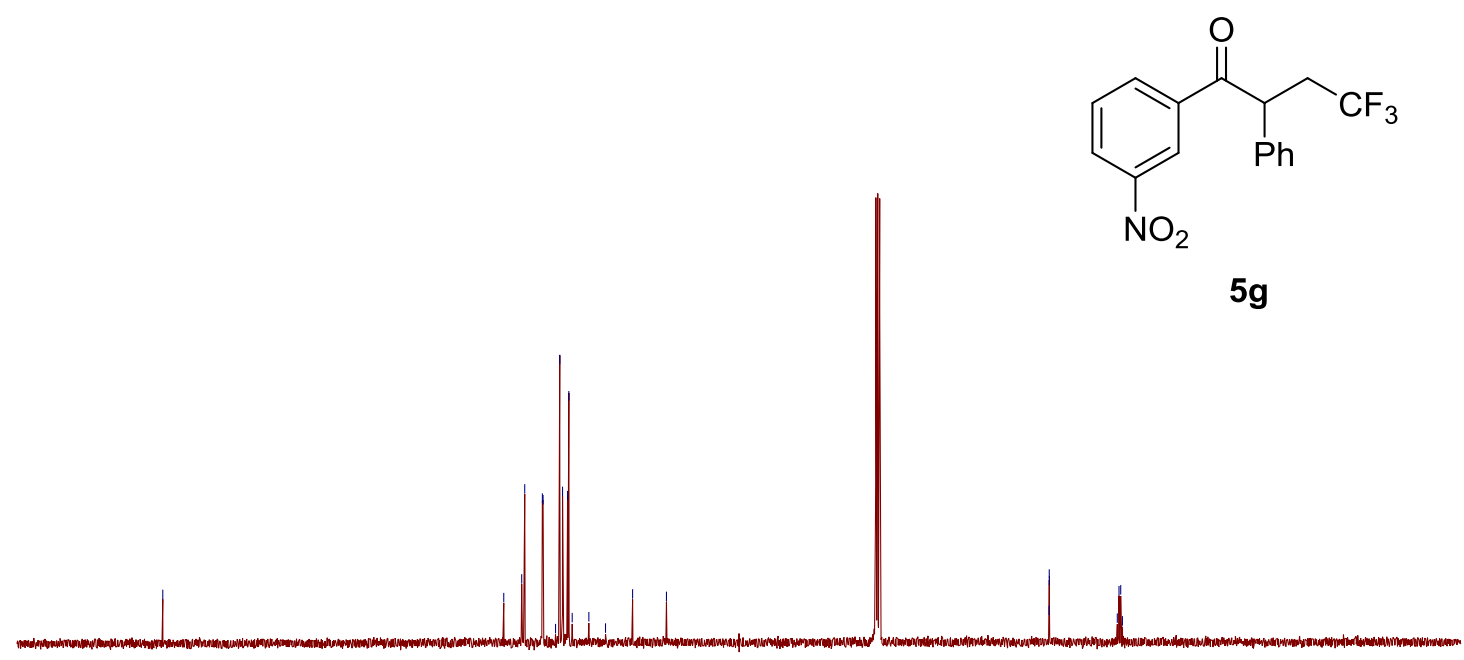




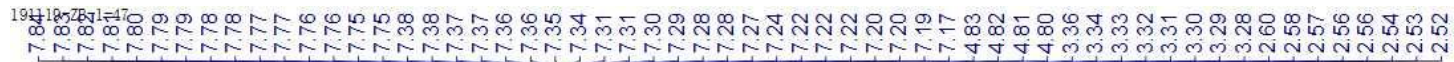

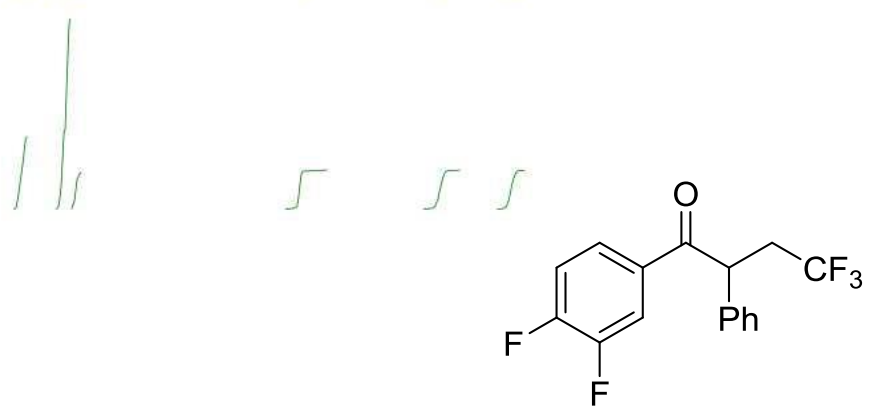

$5 h$

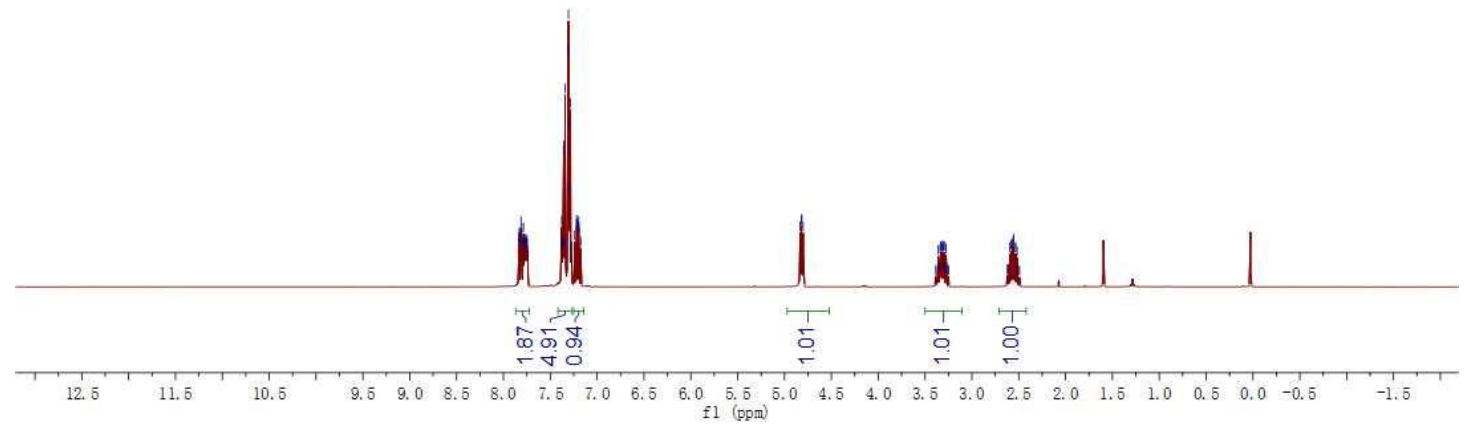

191119-ZB-1-47

๑

$\stackrel{\infty}{\infty} \underset{\substack{\infty \\ \infty}}{\infty}$

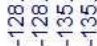<smiles>O=C(c1ccc(F)c(F)c1)C(CC(F)(F)F)c1ccccc1</smiles>

$5 \mathrm{~h}$

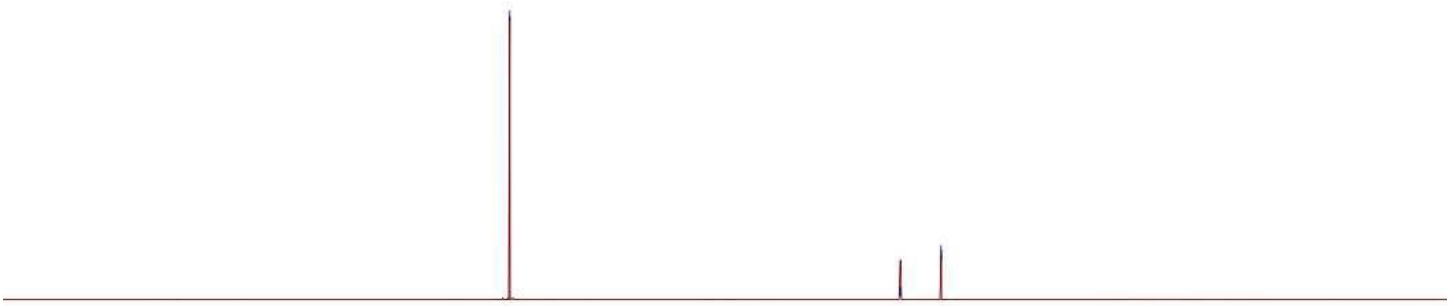


<smiles>O=C(c1ccc(F)c(F)c1)C(CC(F)(F)F)c1ccccc1</smiles>

$5 \mathrm{~h}$
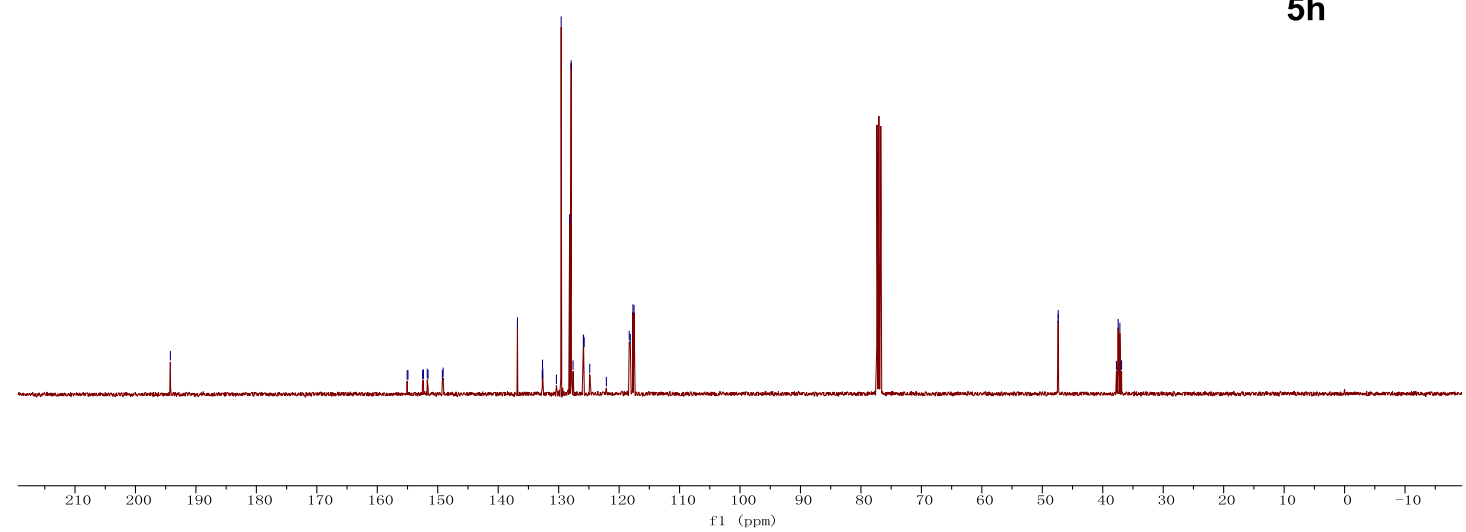


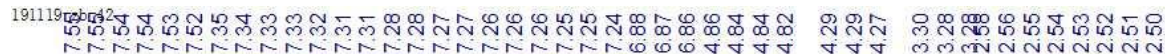
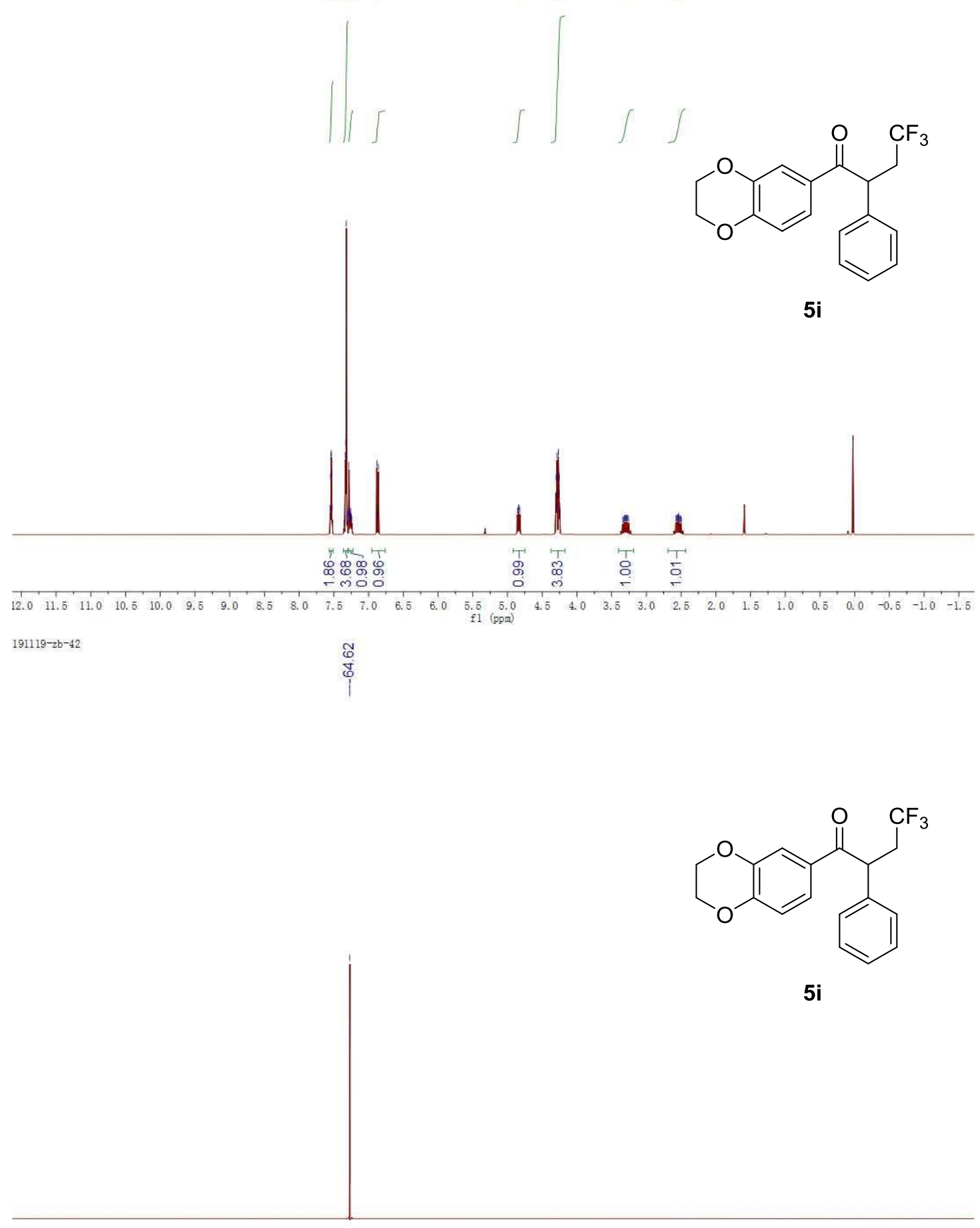

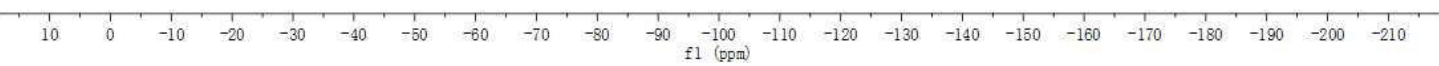



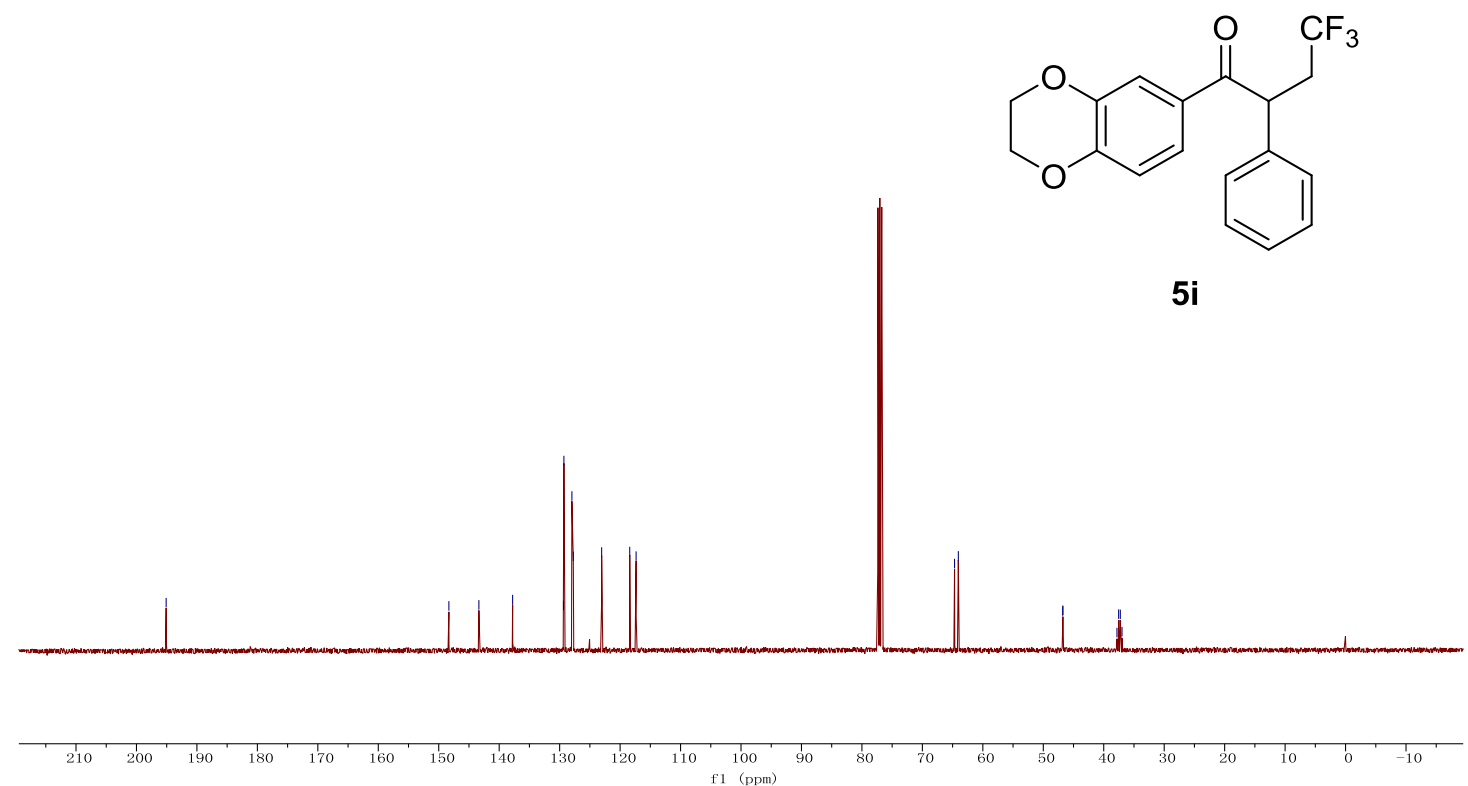


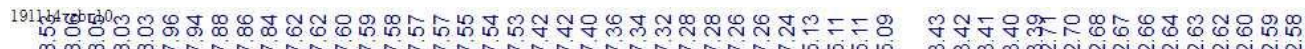

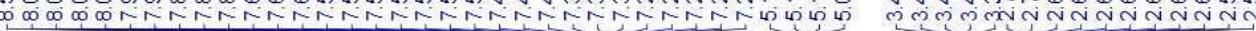

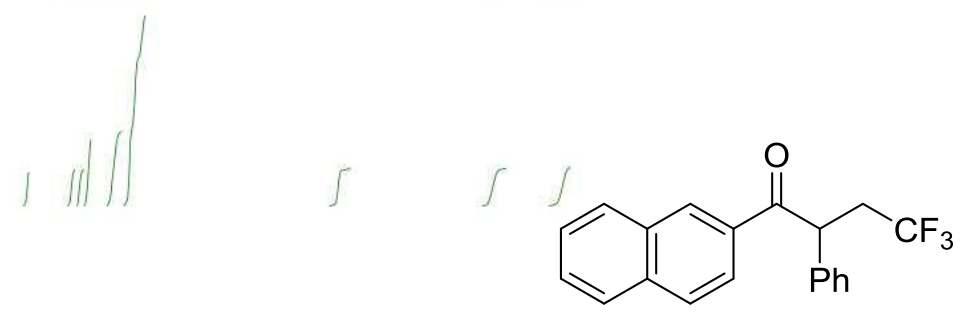

$5 \mathbf{j}$

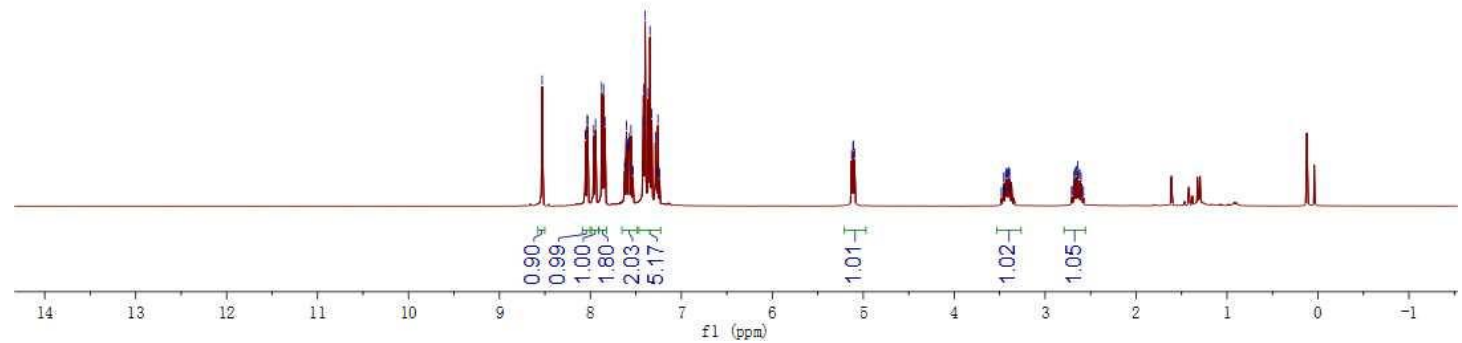

191114-zb-10

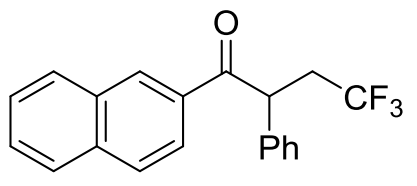

$5 \mathbf{j}$

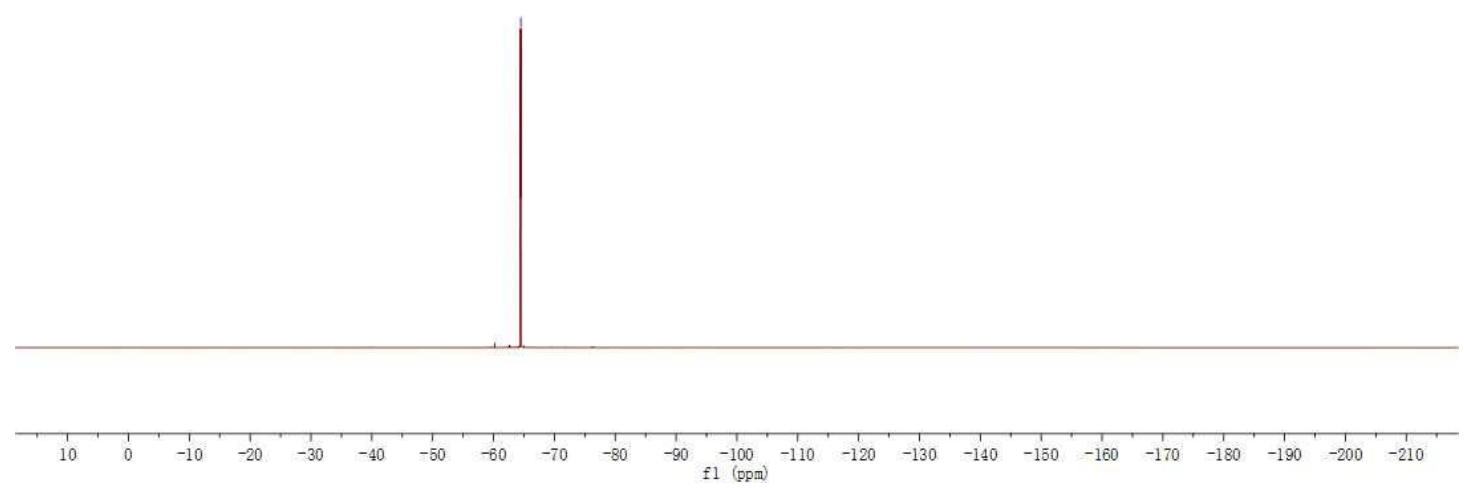


<smiles>O=C(c1ccc2ccccc2c1)C(CC(F)(F)F)c1ccccc1</smiles>

5j

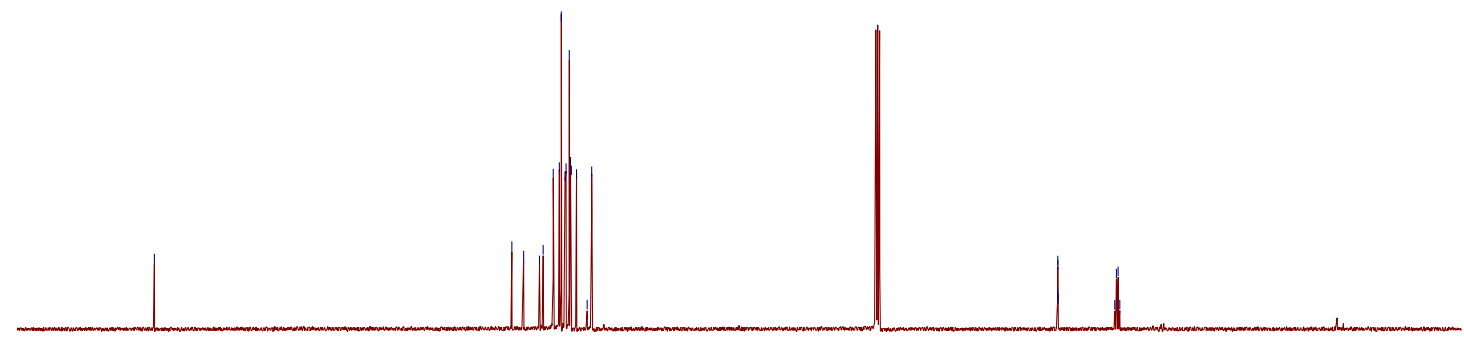

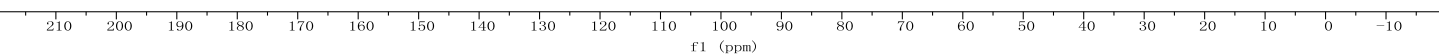




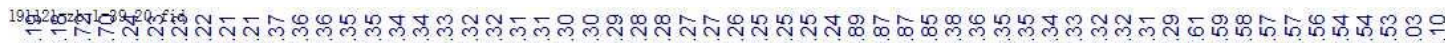

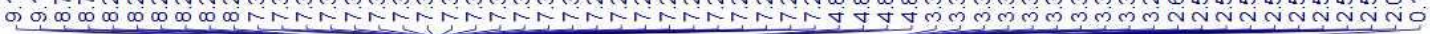

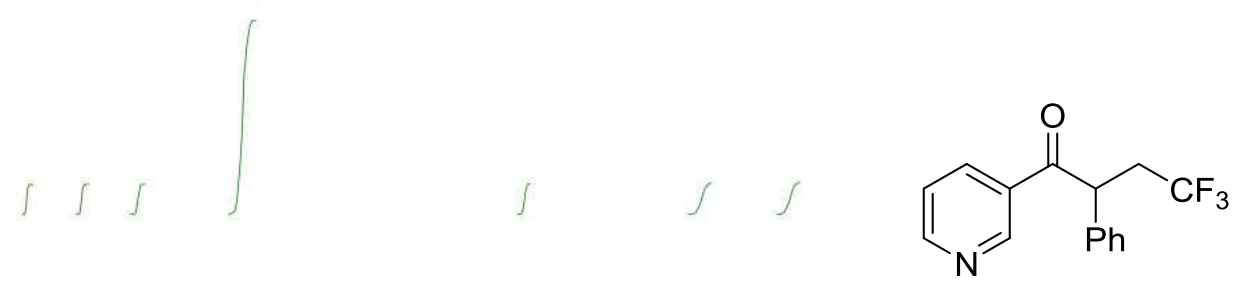

$5 k$

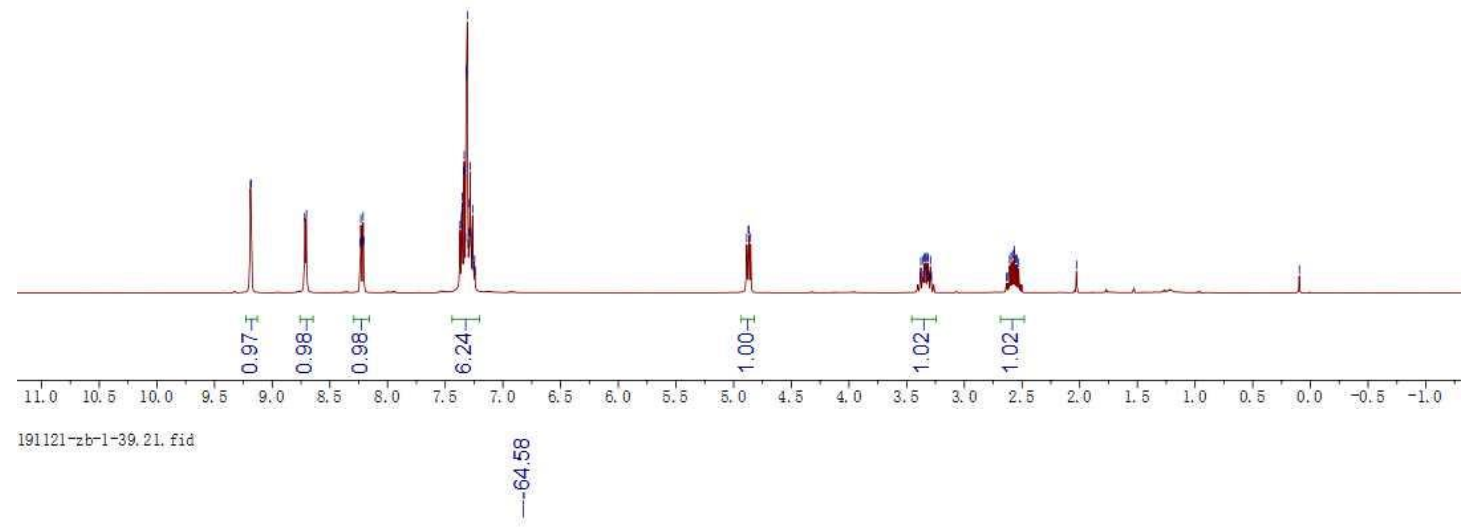<smiles>O=C(c1cccnc1)C(CC(F)(F)F)c1ccccc1</smiles>

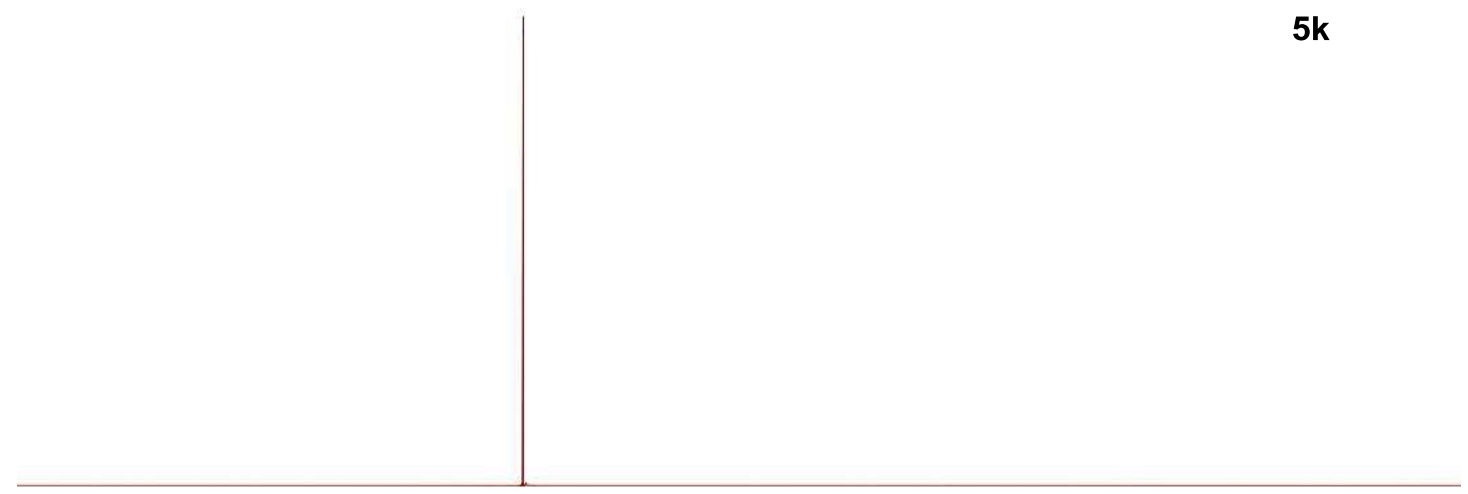

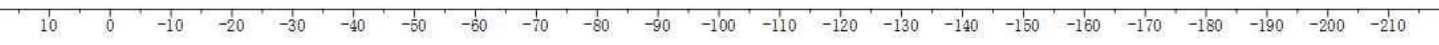




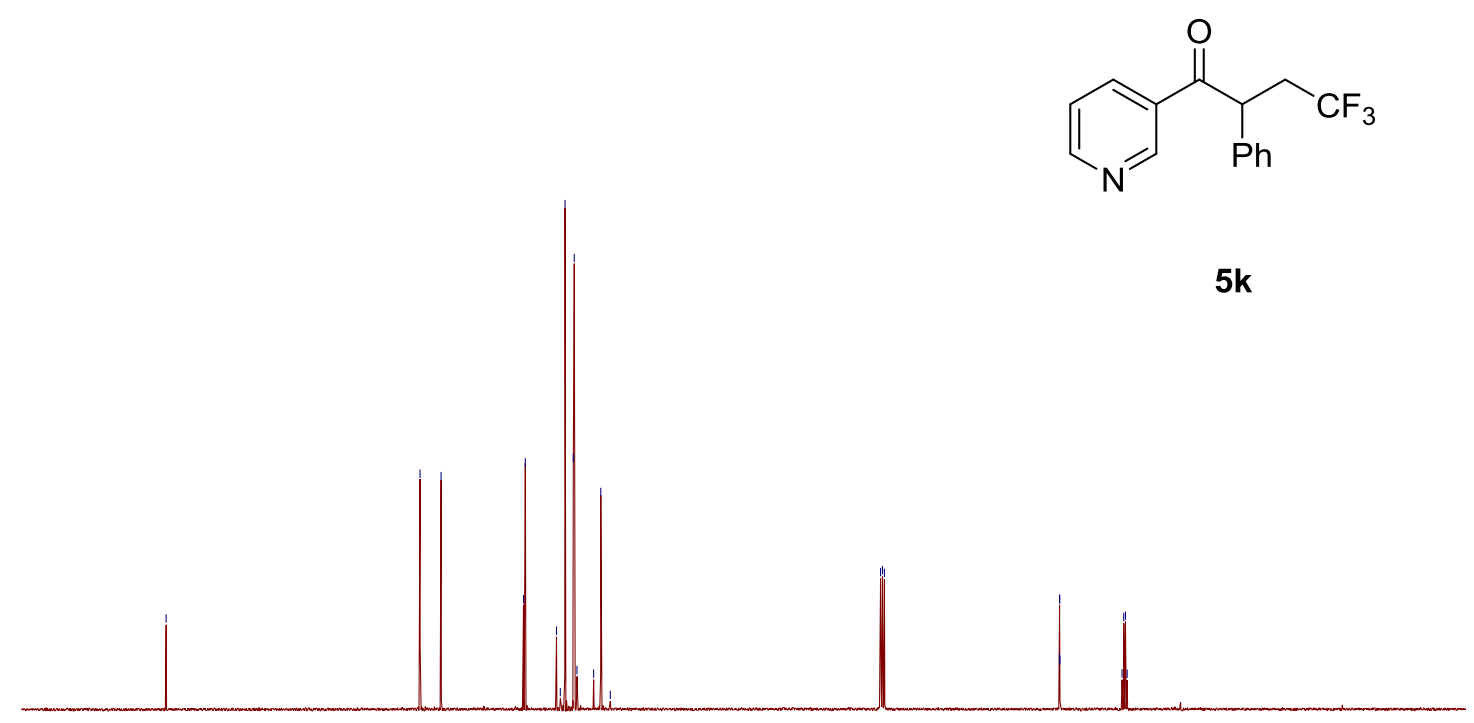



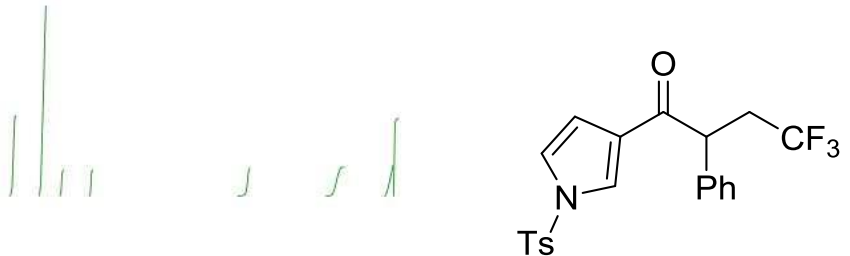

5

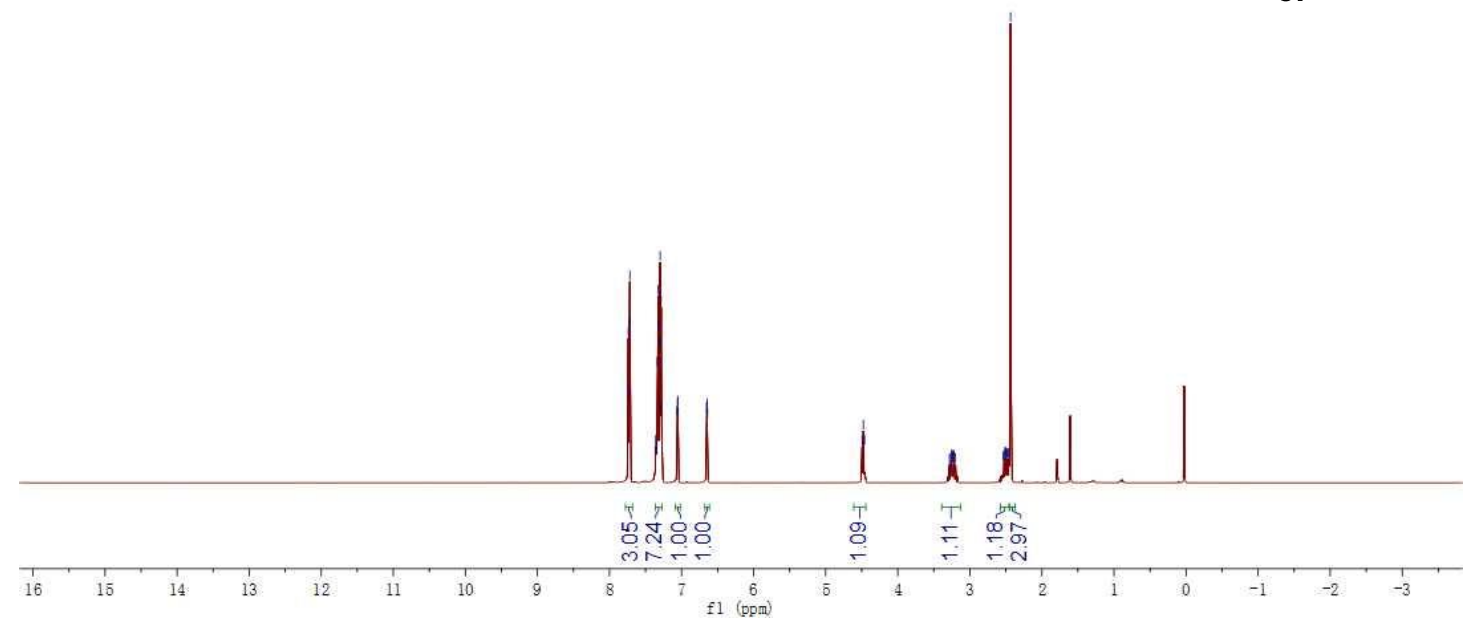

$191115-z b-15$

$\infty$
1
$\mathbb{1}$

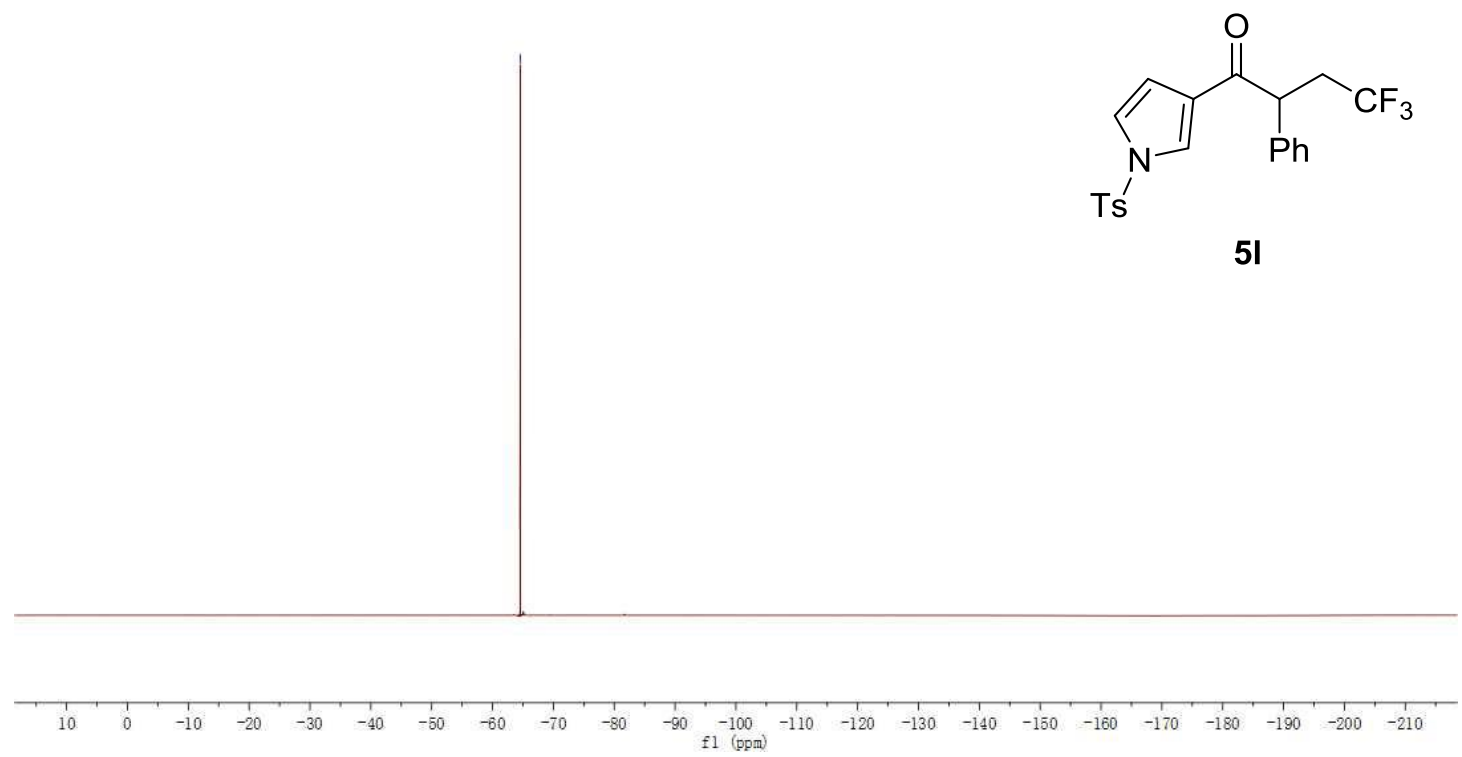



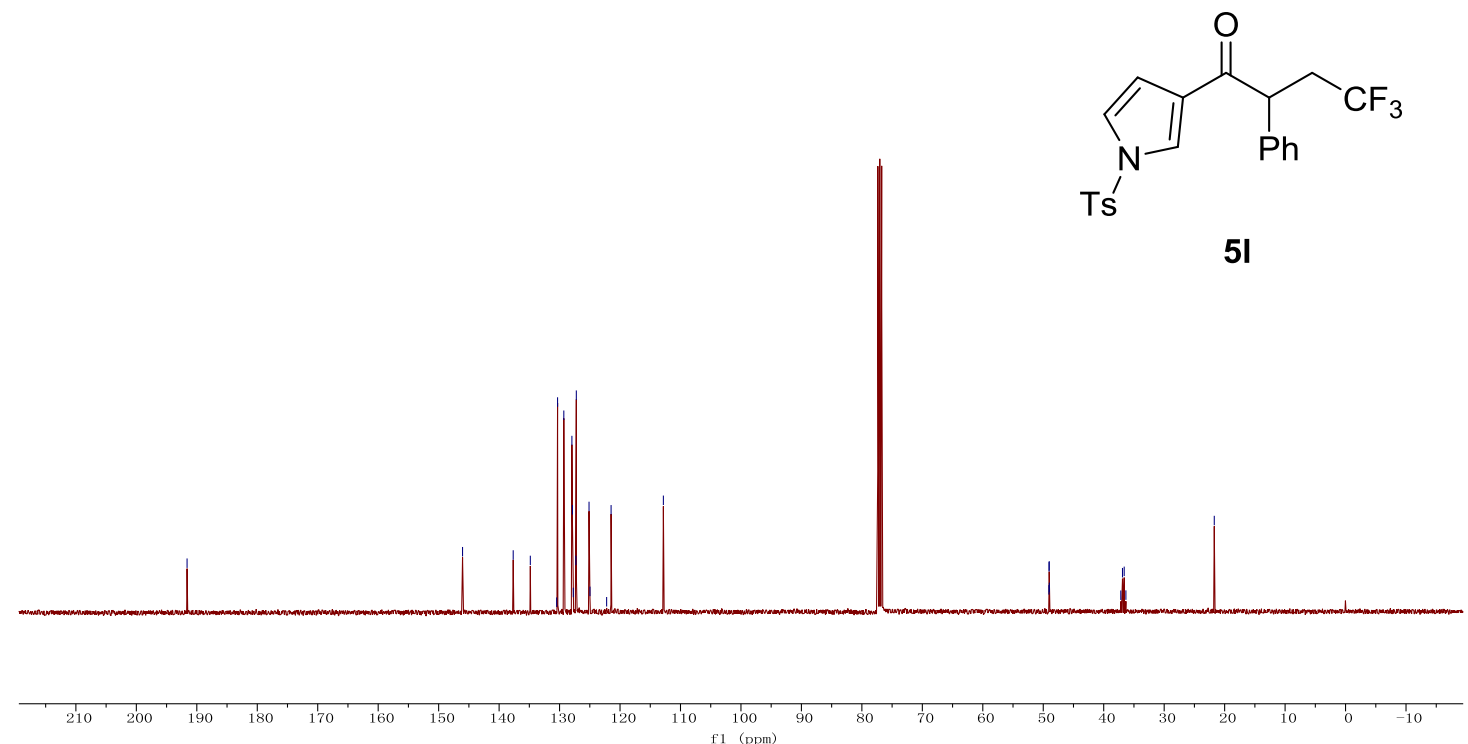

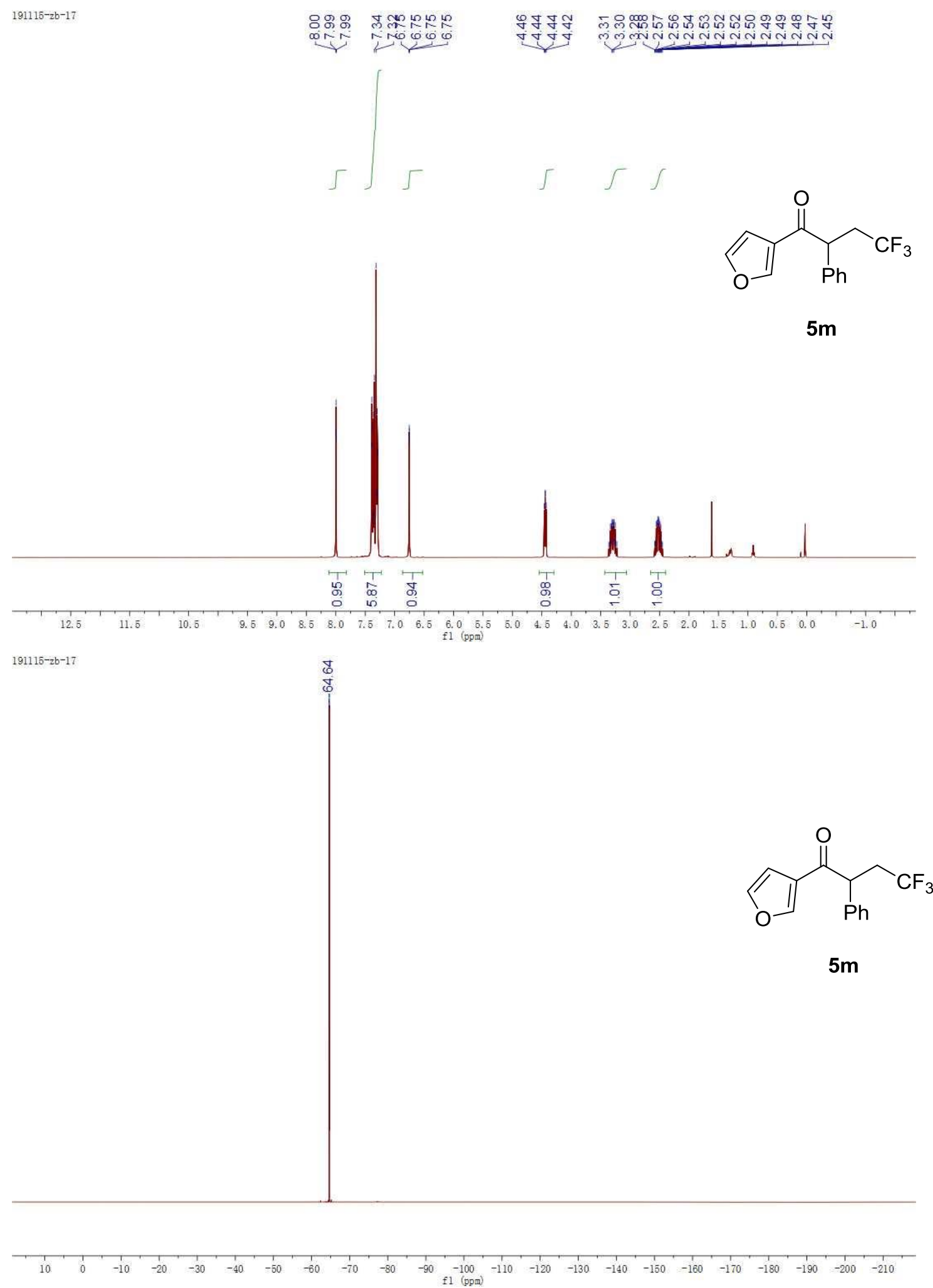


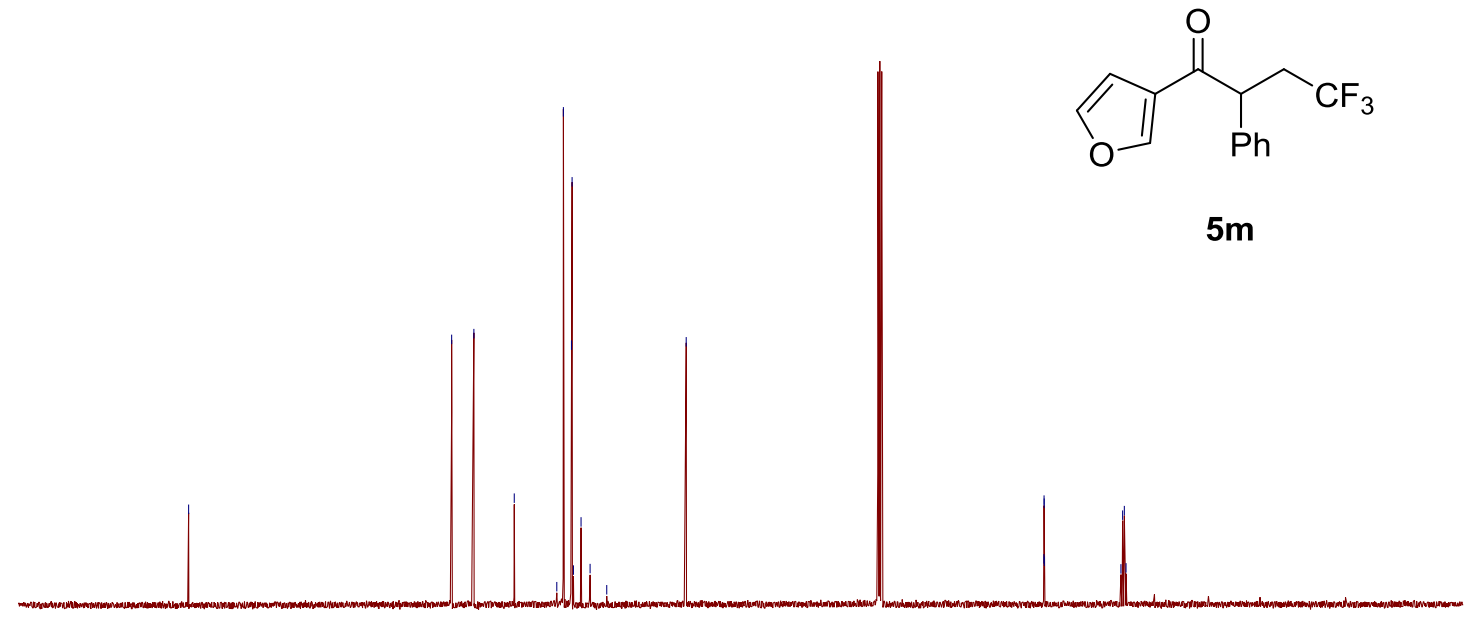




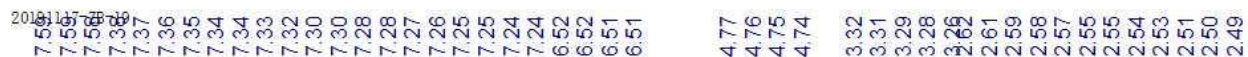

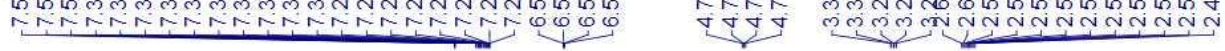
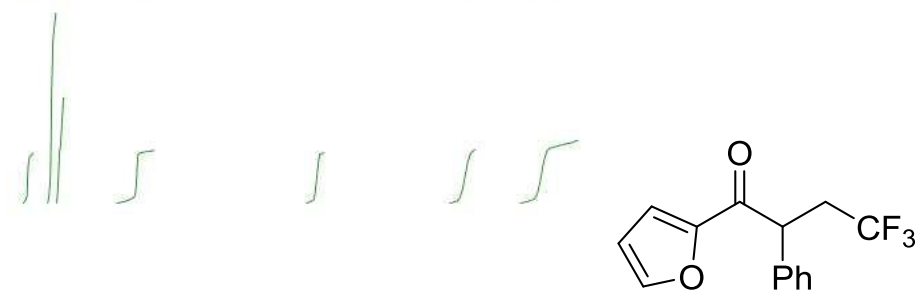

$5 n$

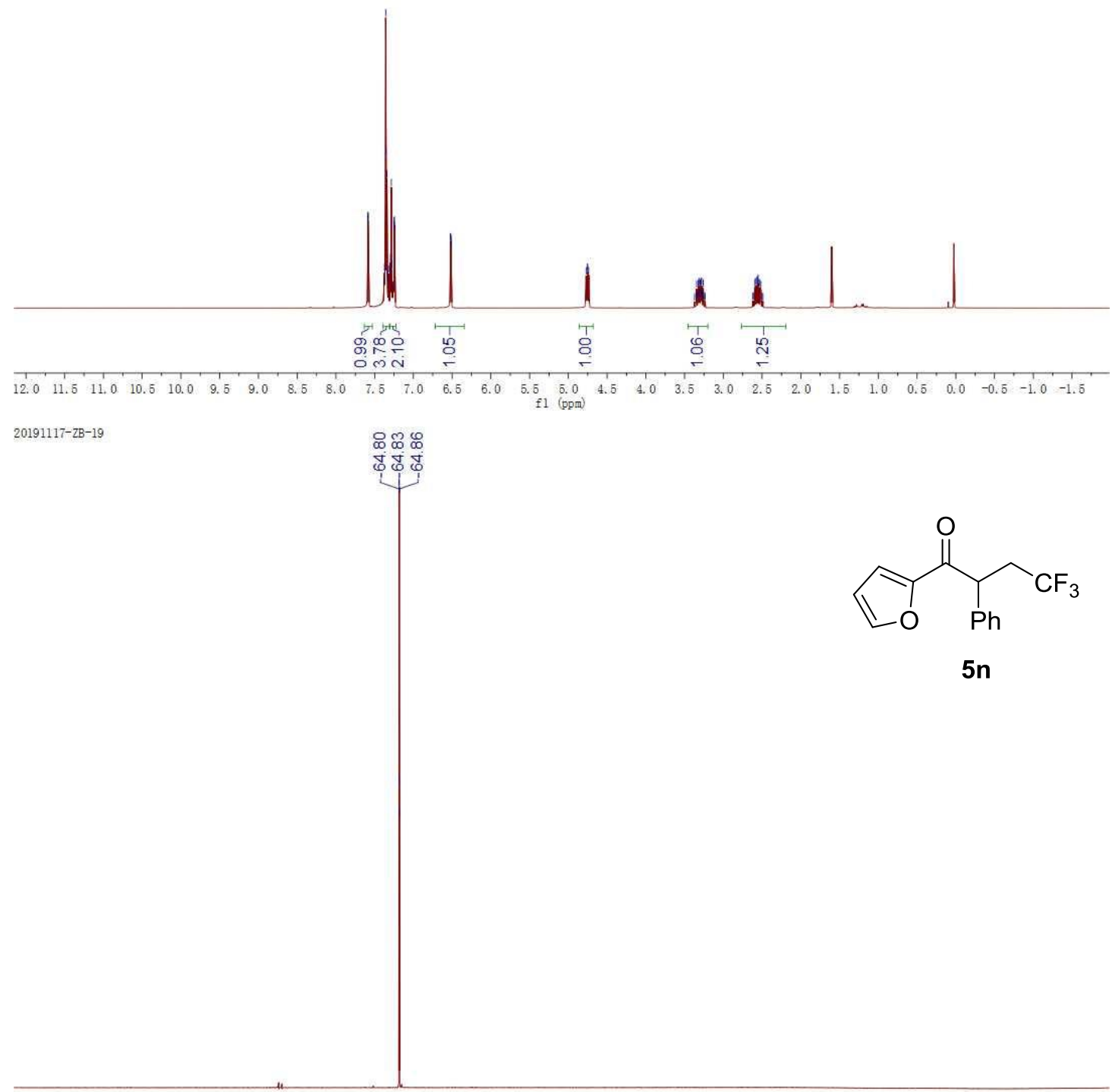

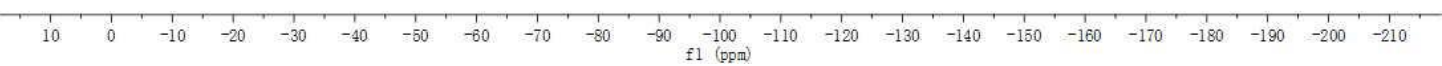


20191117-ZB-19

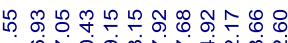

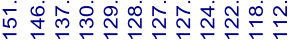

(1)
กุำ

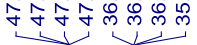

$5 n$

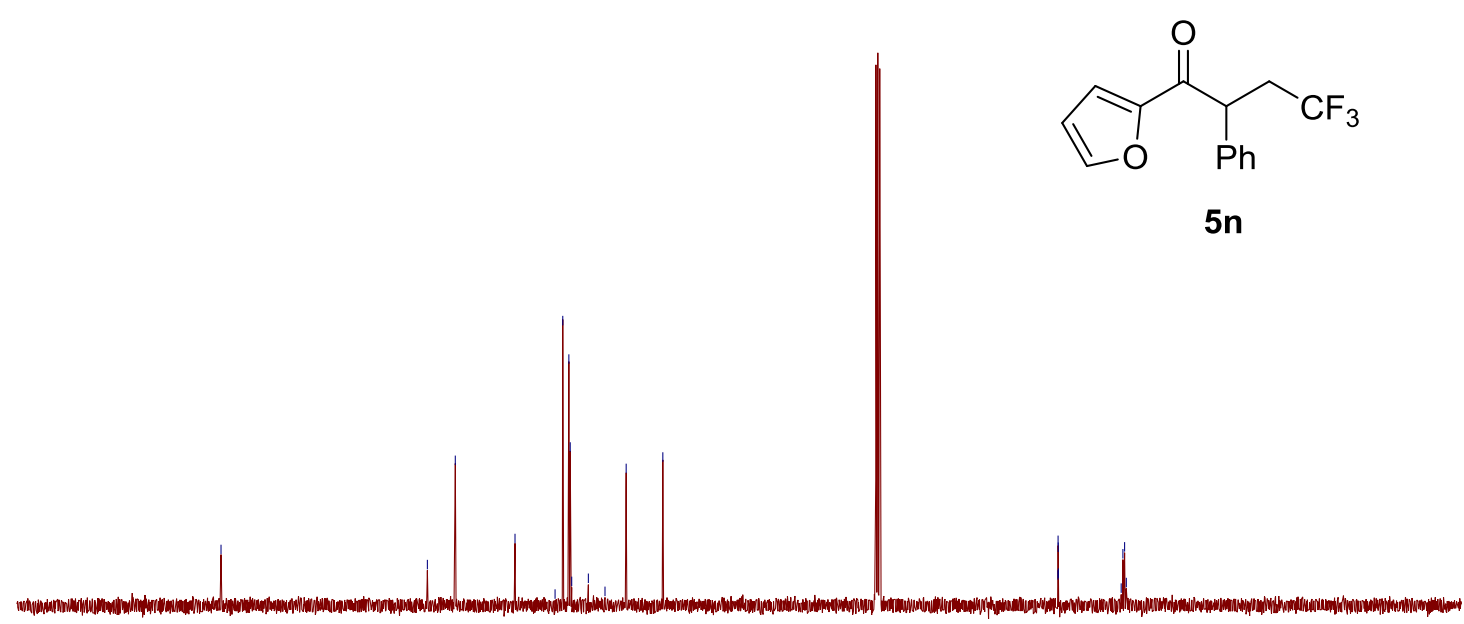




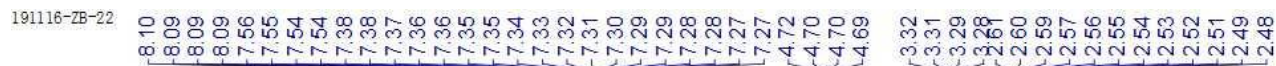

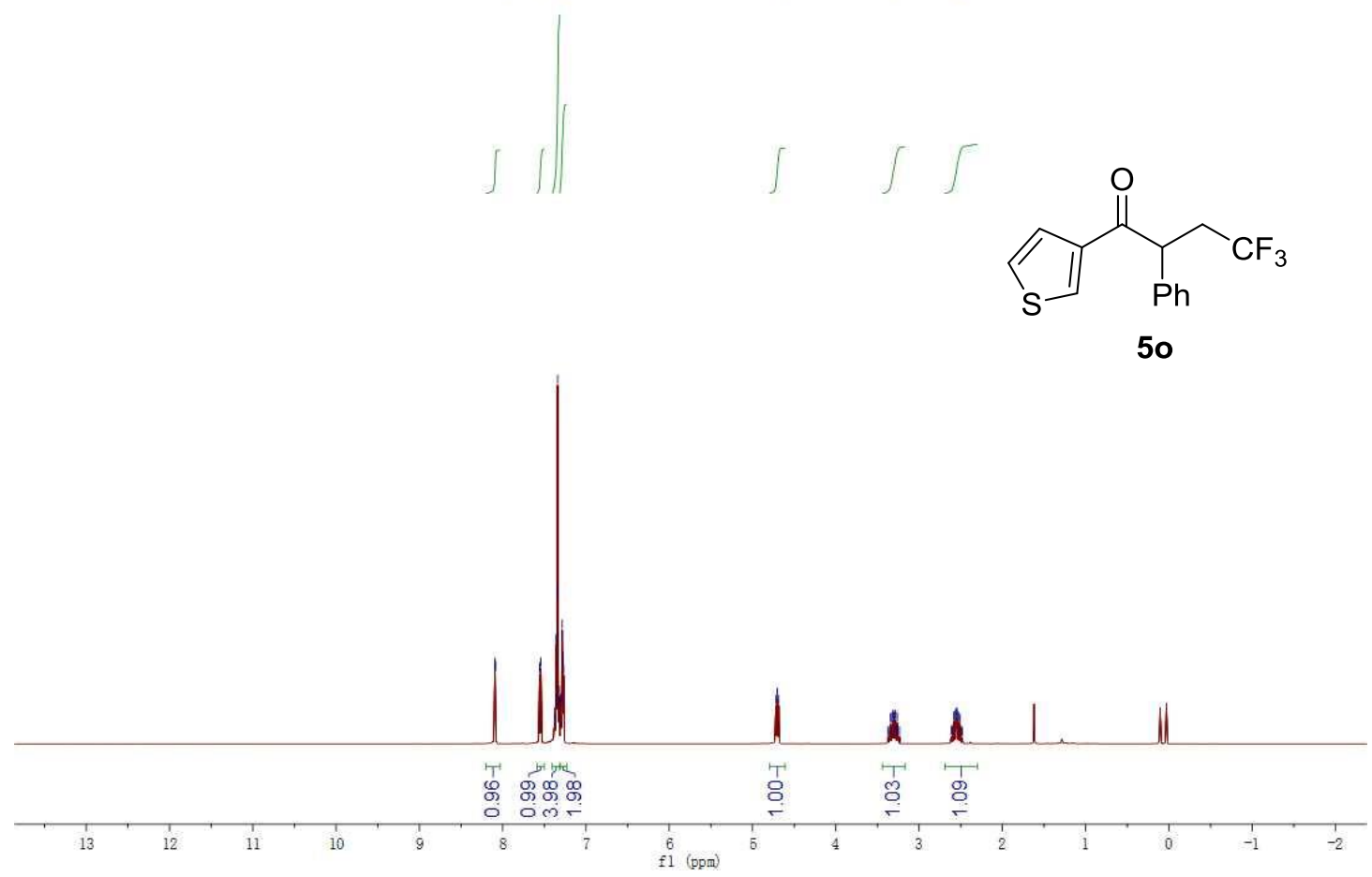

$191116-2 B-22$

|
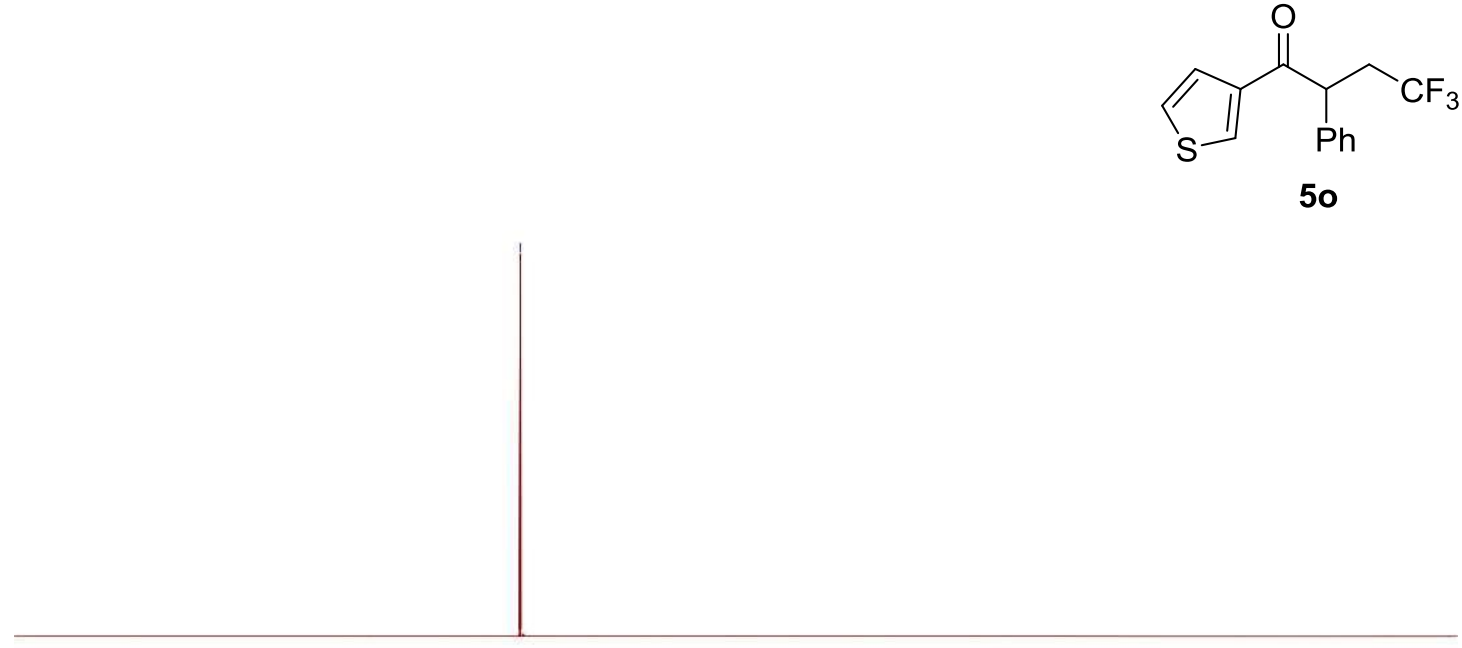

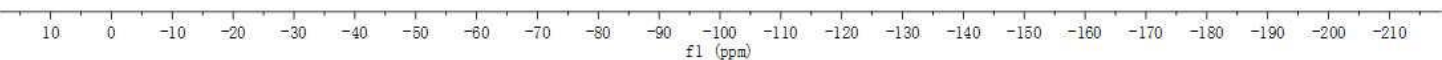


191116-ZB-22

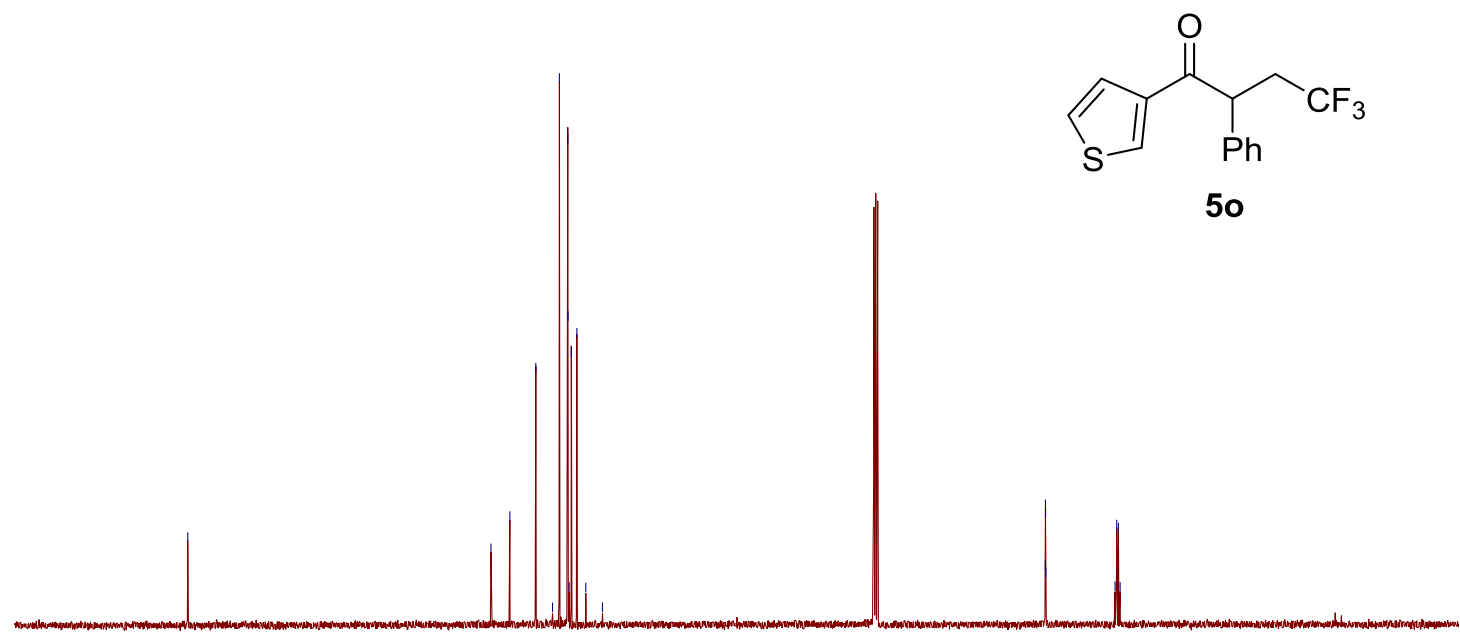

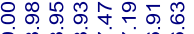

ز

50

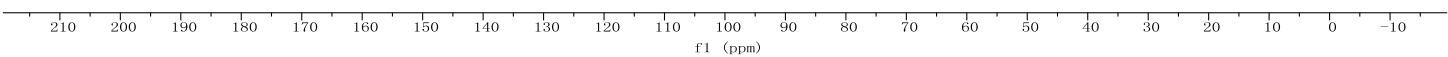




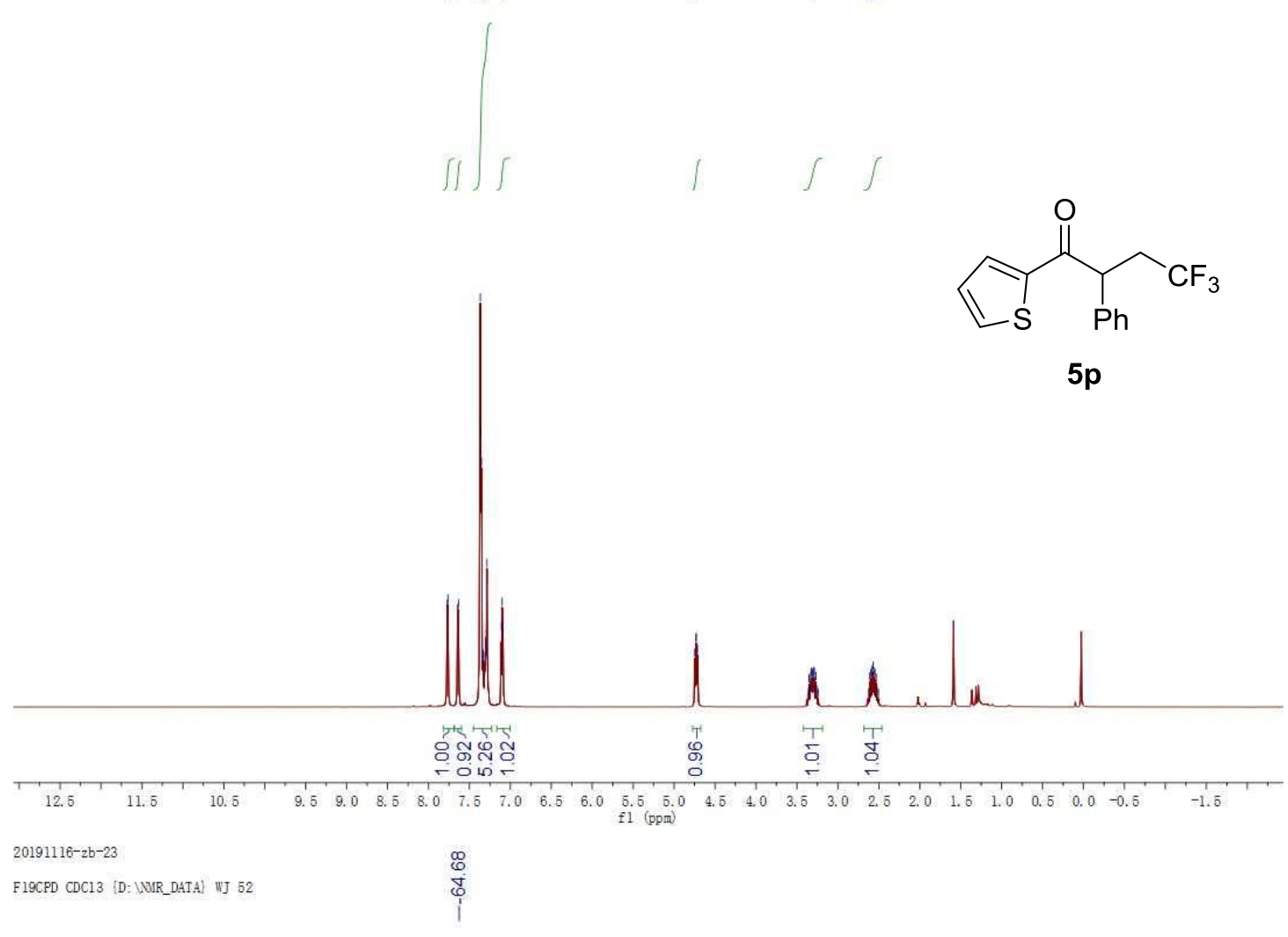

20191116-zb-23

F19CPD CDC13 \{D: WNR_DATA\} WJ 52

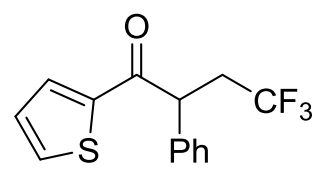

$5 p$

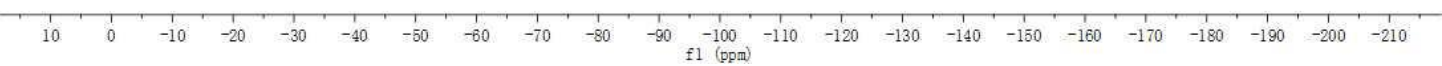


$20191116-\mathrm{zb}-23$

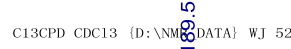

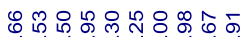

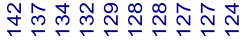

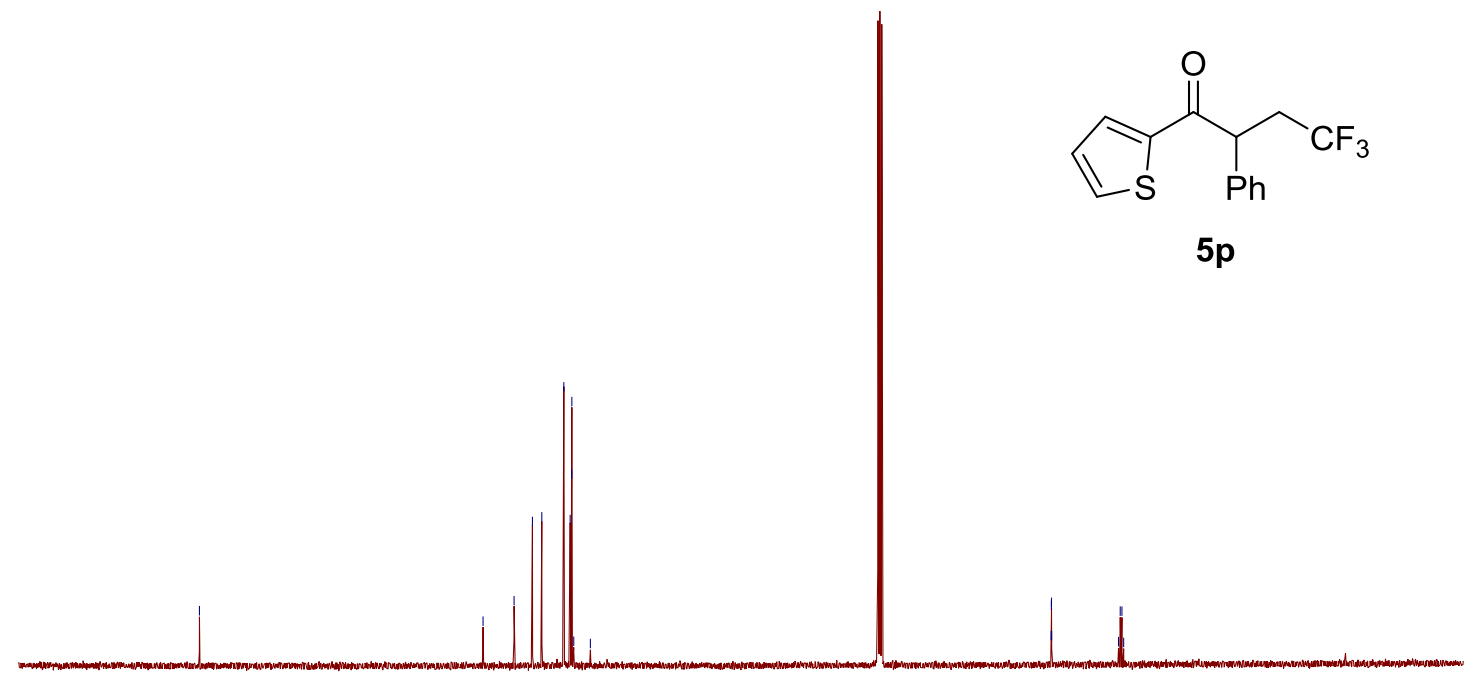

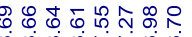

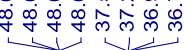

$5 p$

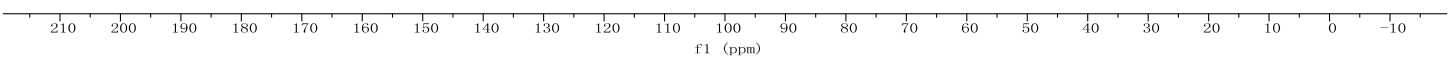



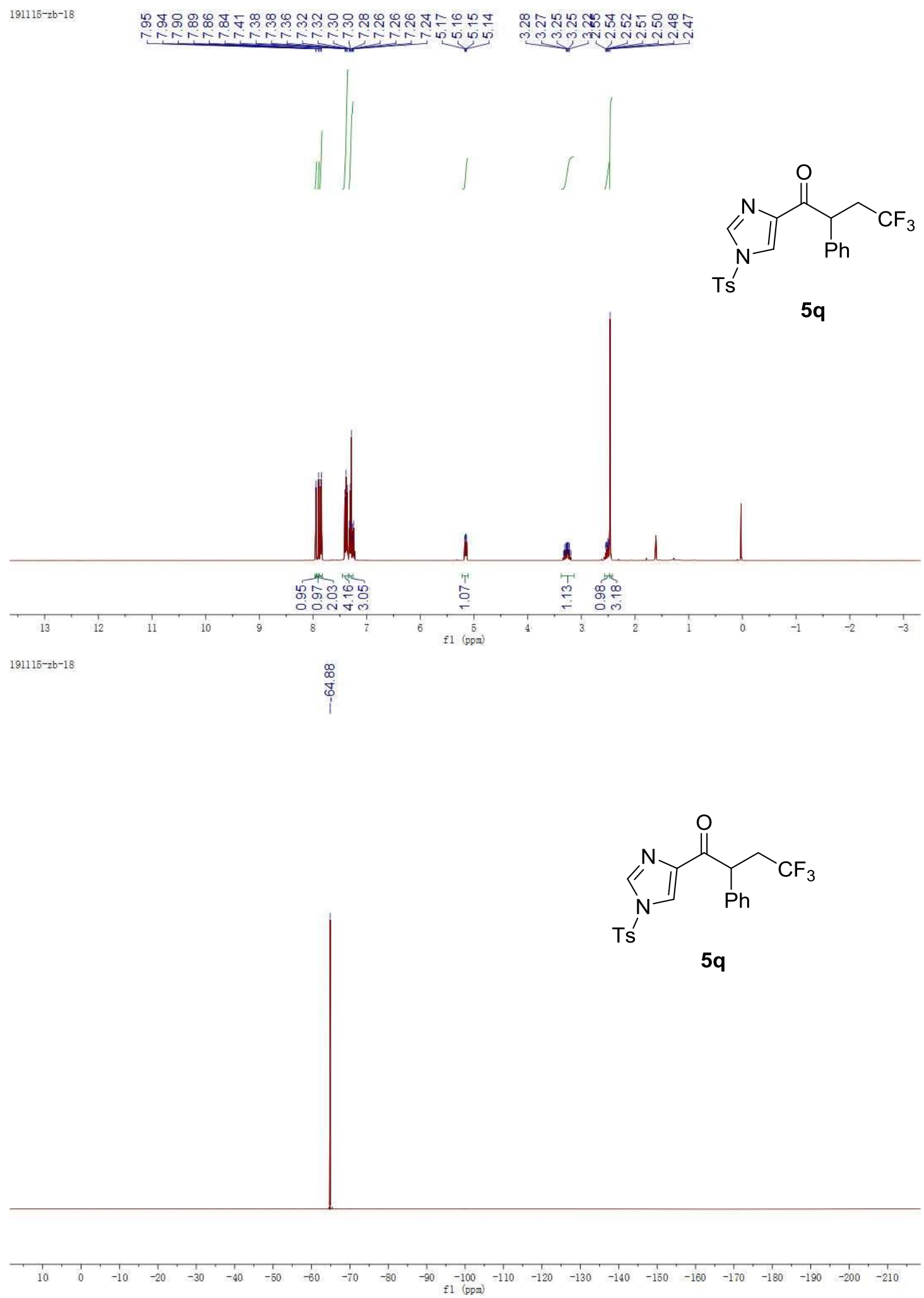
191115-zb-18

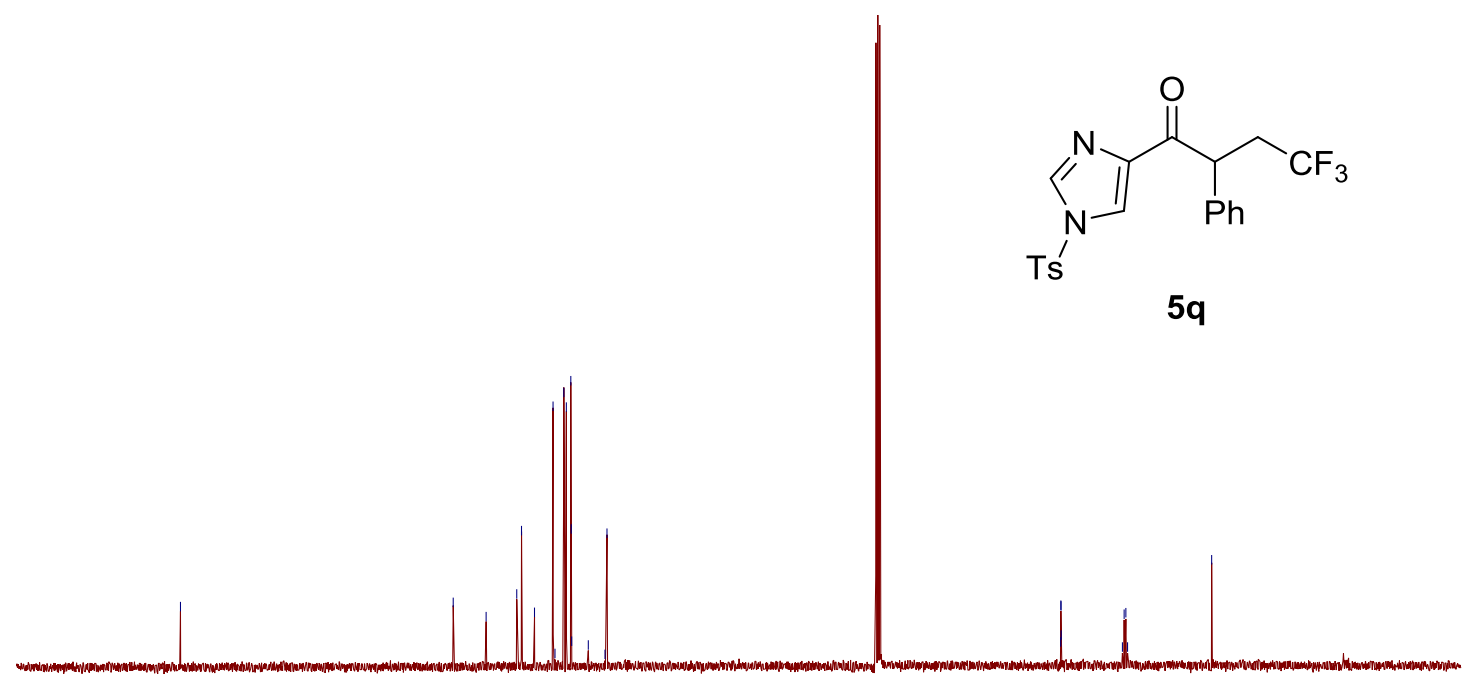

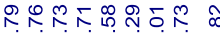

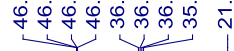

$5 q$ 


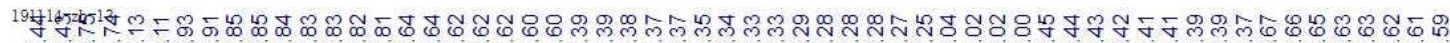

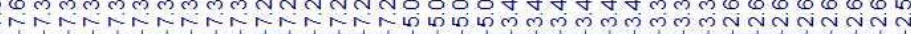
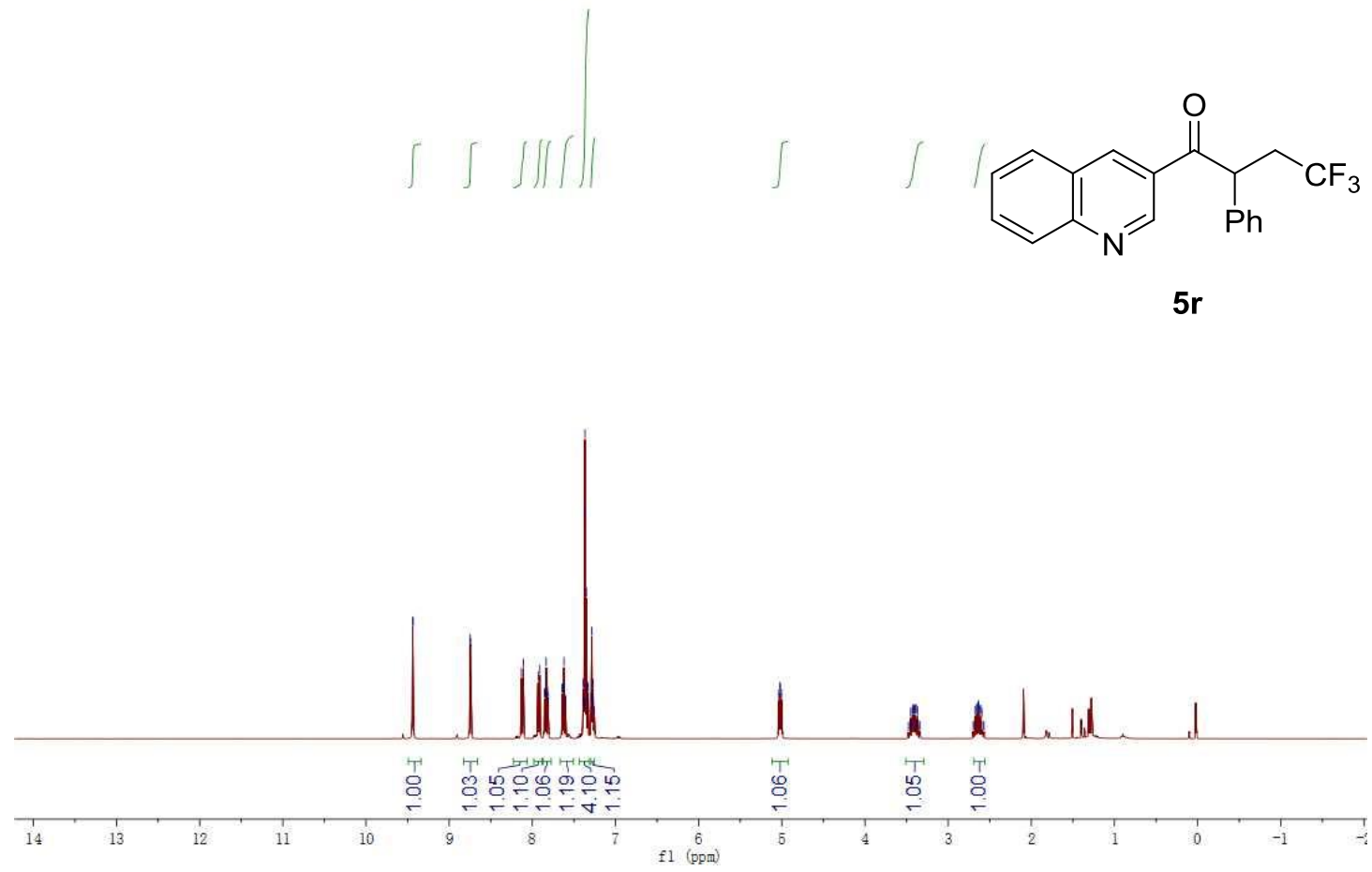

$191115-z b-13$

$\stackrel{\infty}{\stackrel{0}{0}}$

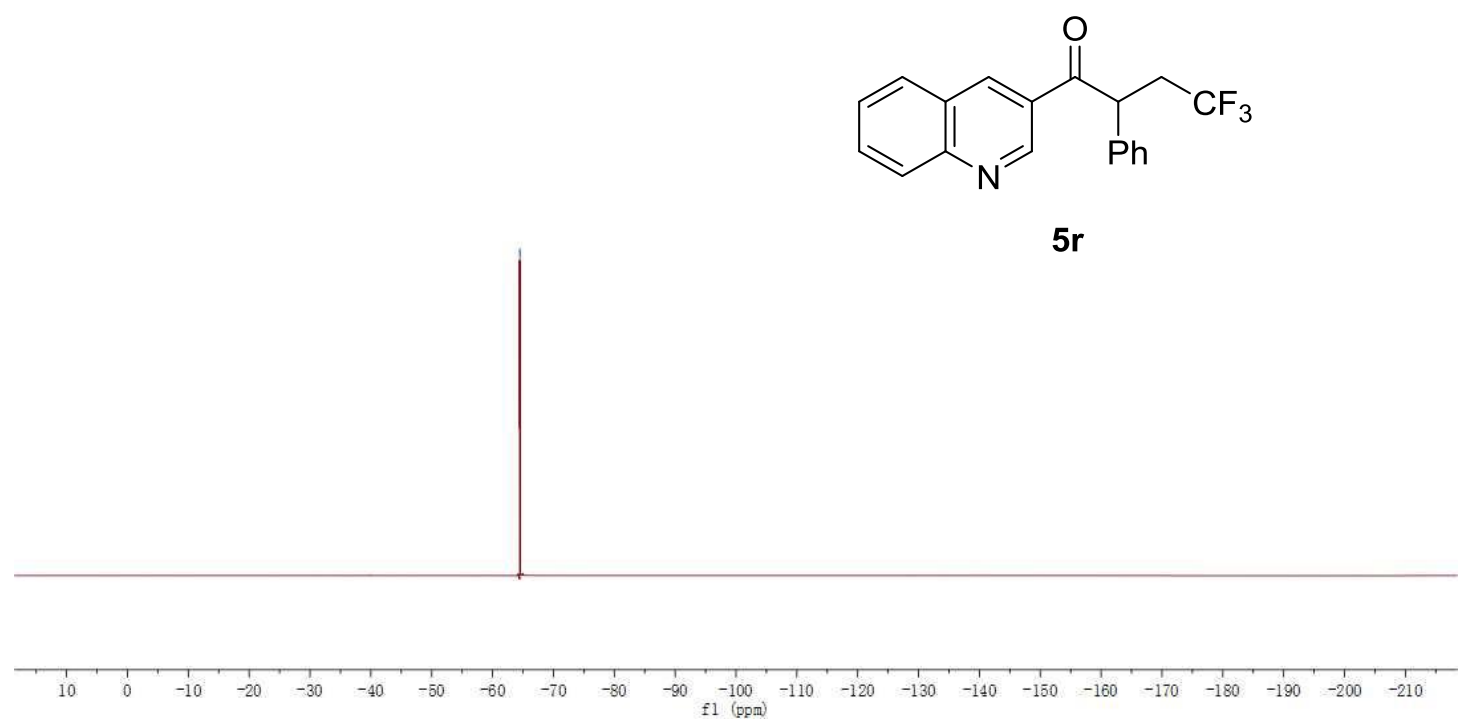



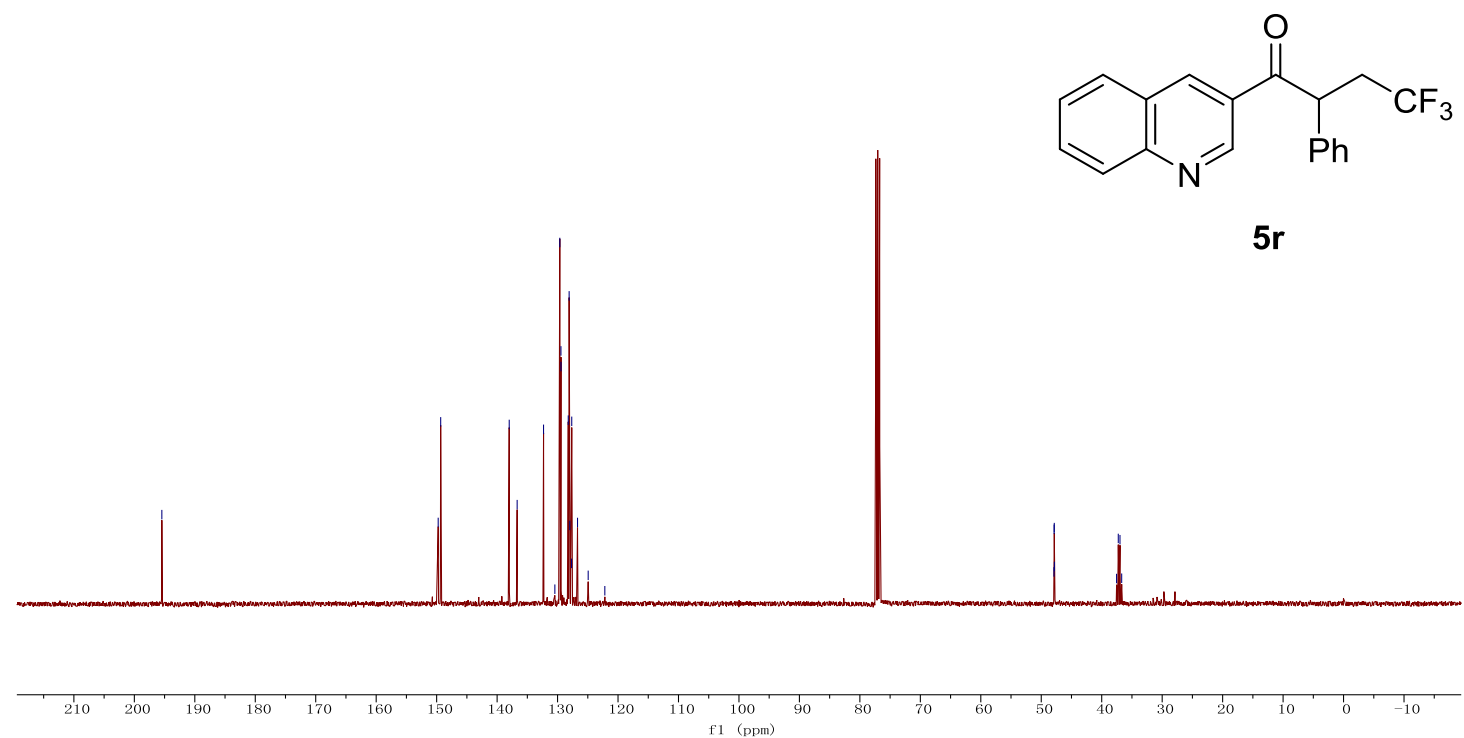


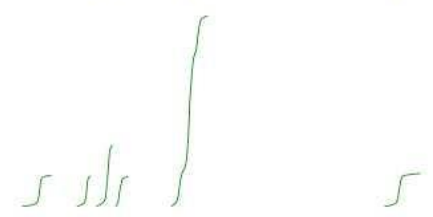<smiles>O=C(c1ccc2ncccc2c1)C(CC(F)(F)F)c1ccccc1</smiles>

$5 s$

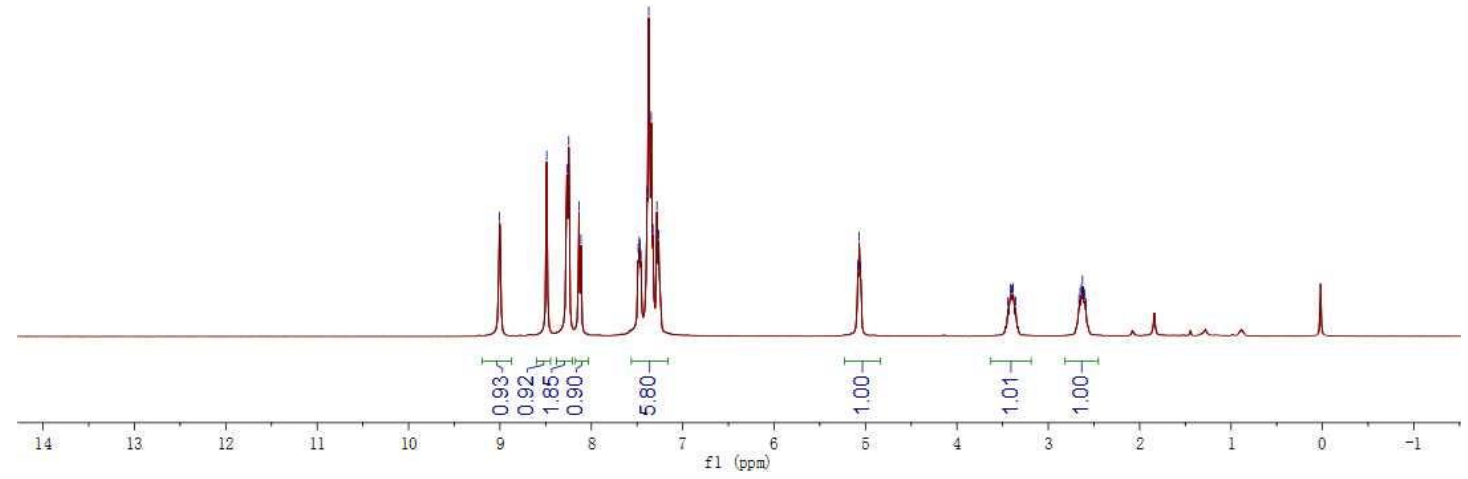

191115-zb-12
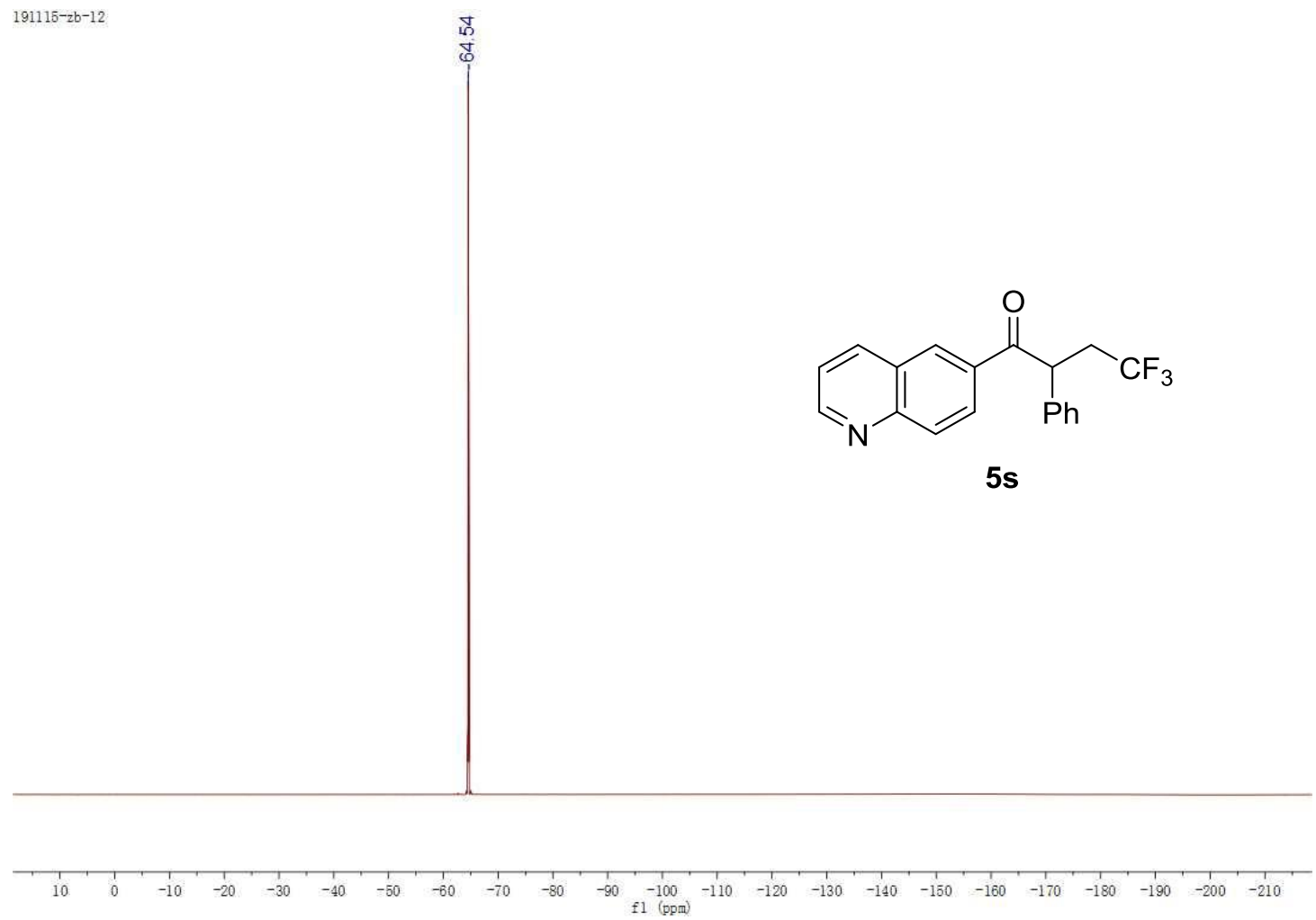


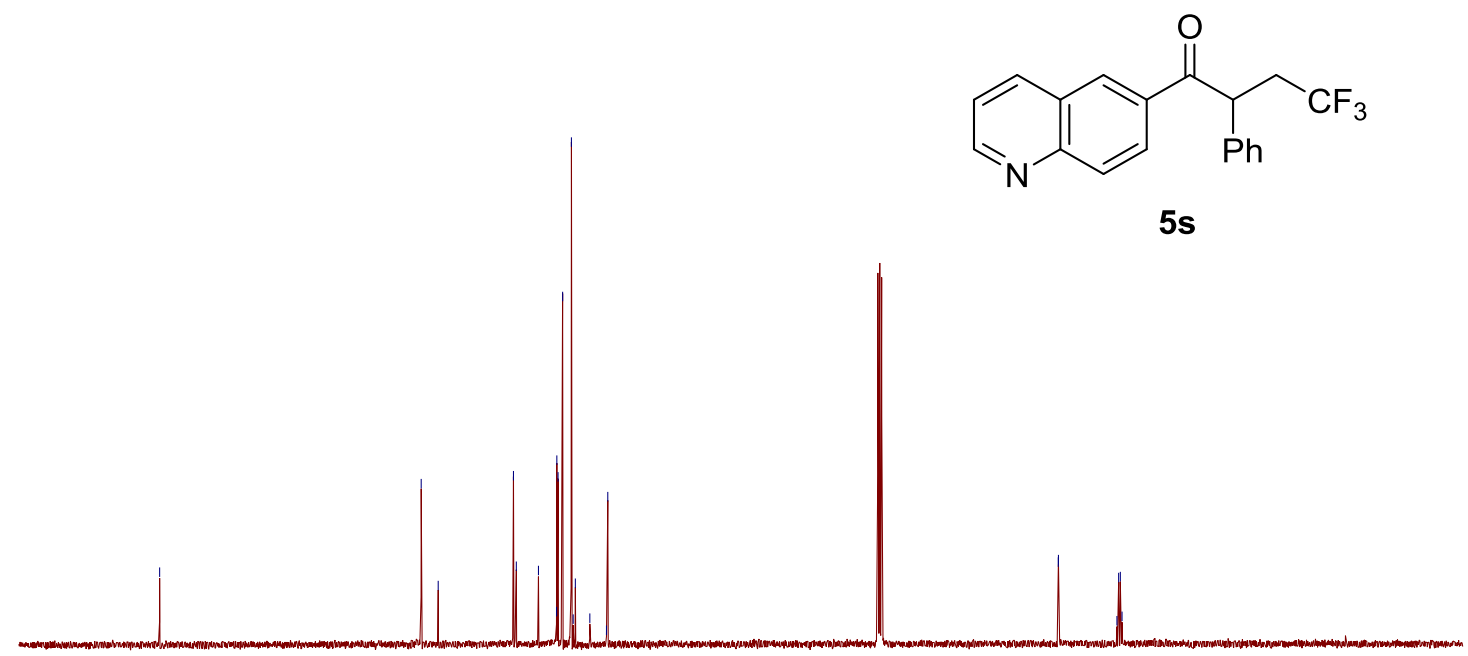




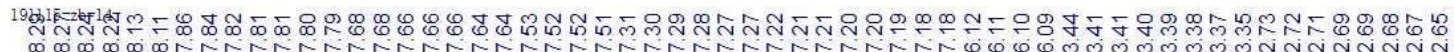

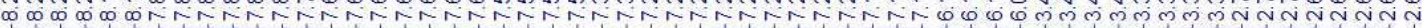
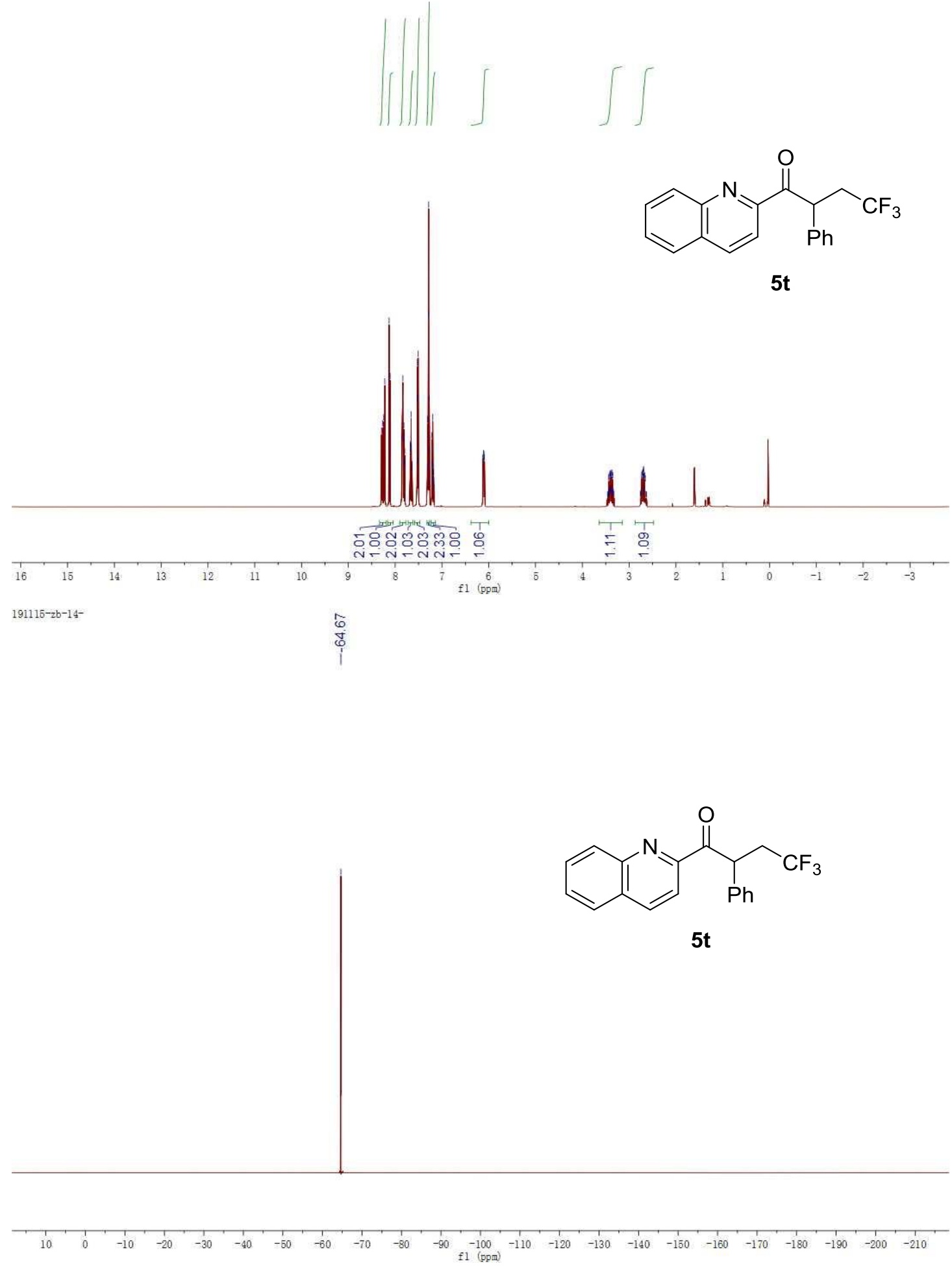

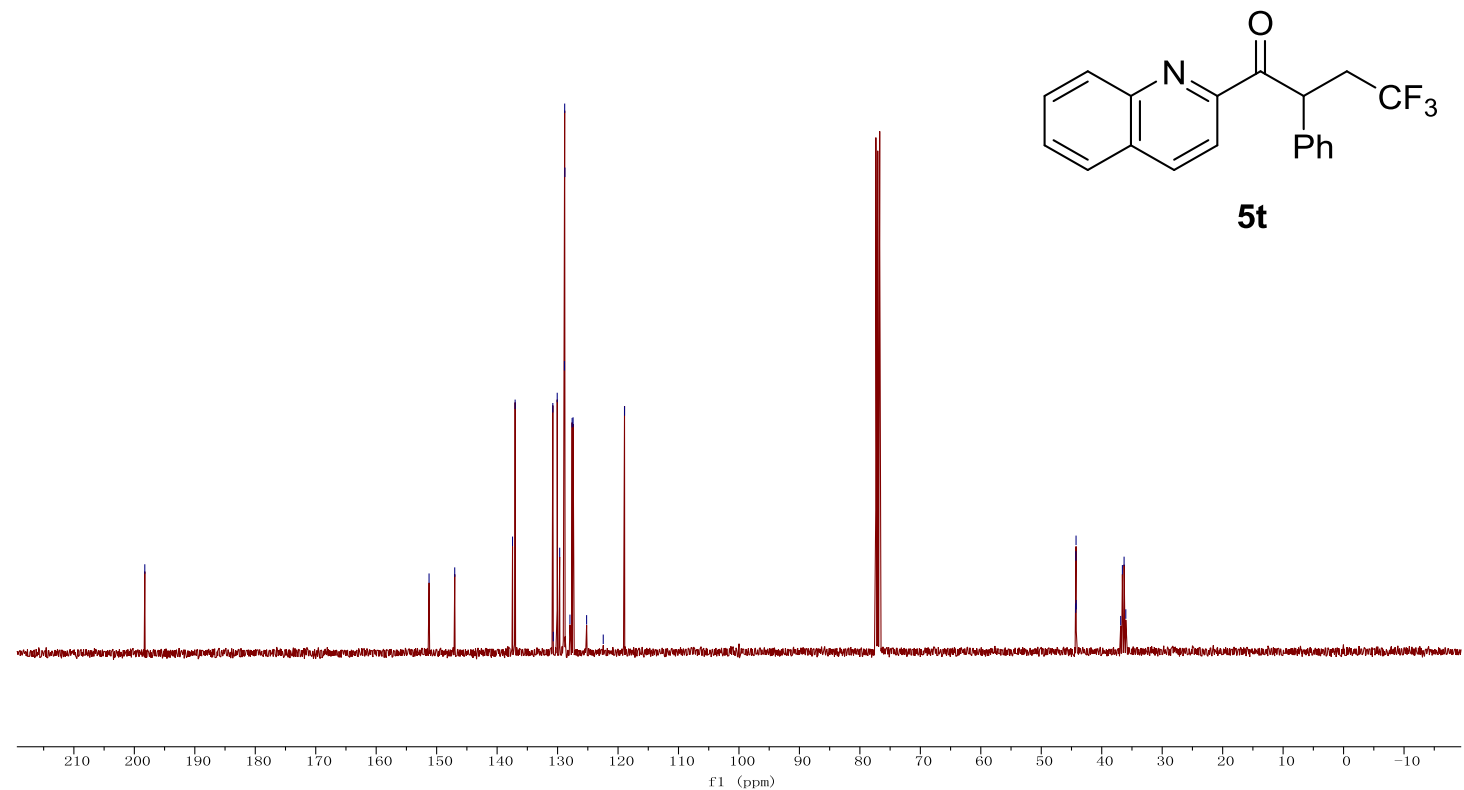


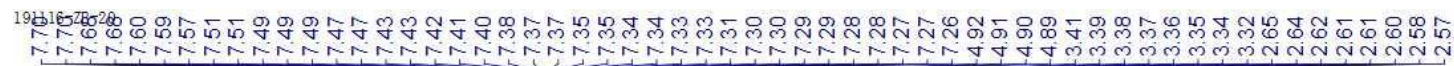

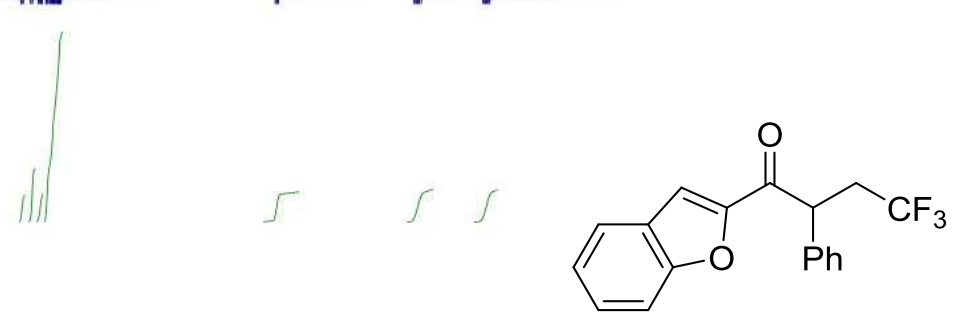

$5 u$

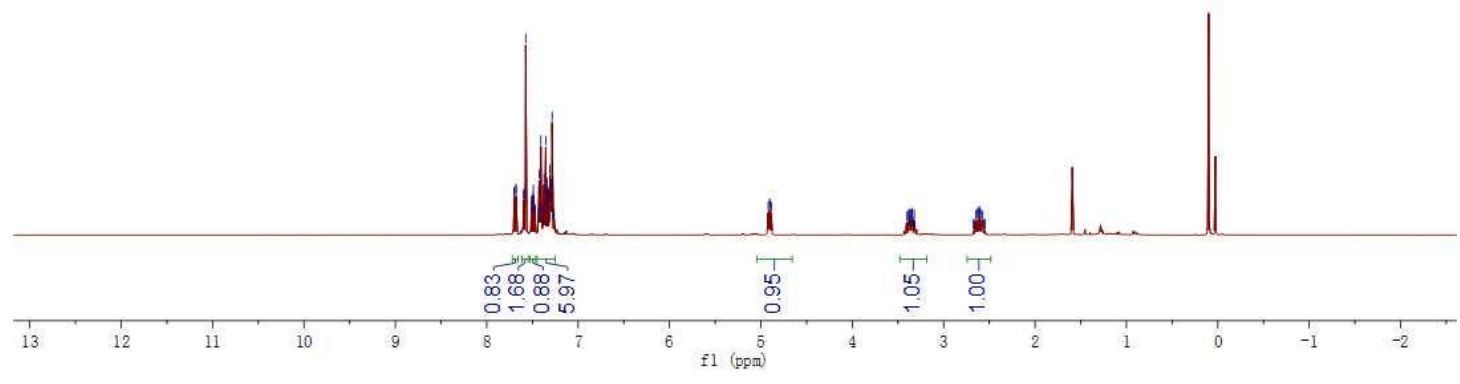

$191116-2 B-20$

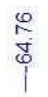<smiles>O=C(c1cc2ccccc2o1)C(CC(F)(F)F)c1ccccc1</smiles>

$5 u$
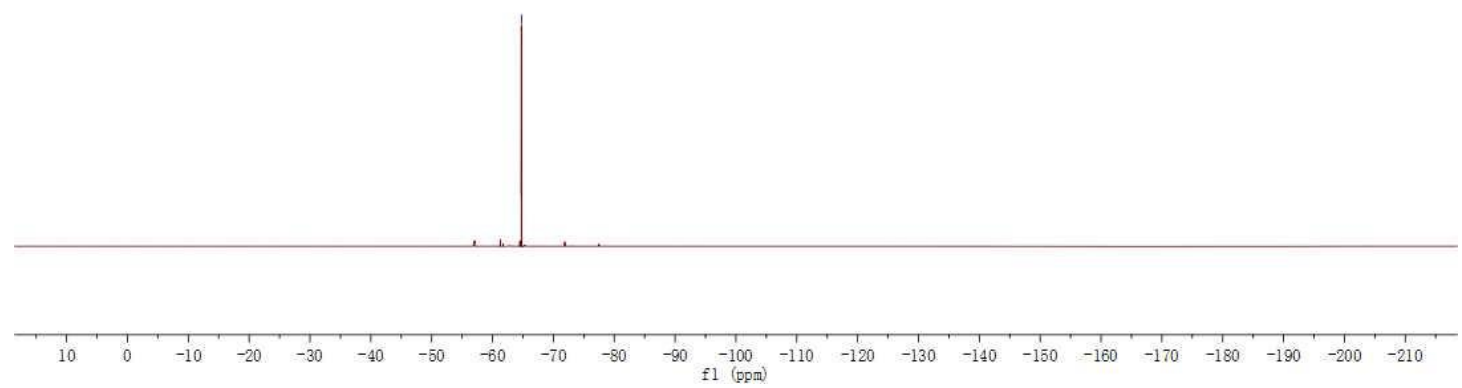
191116-ZB-20
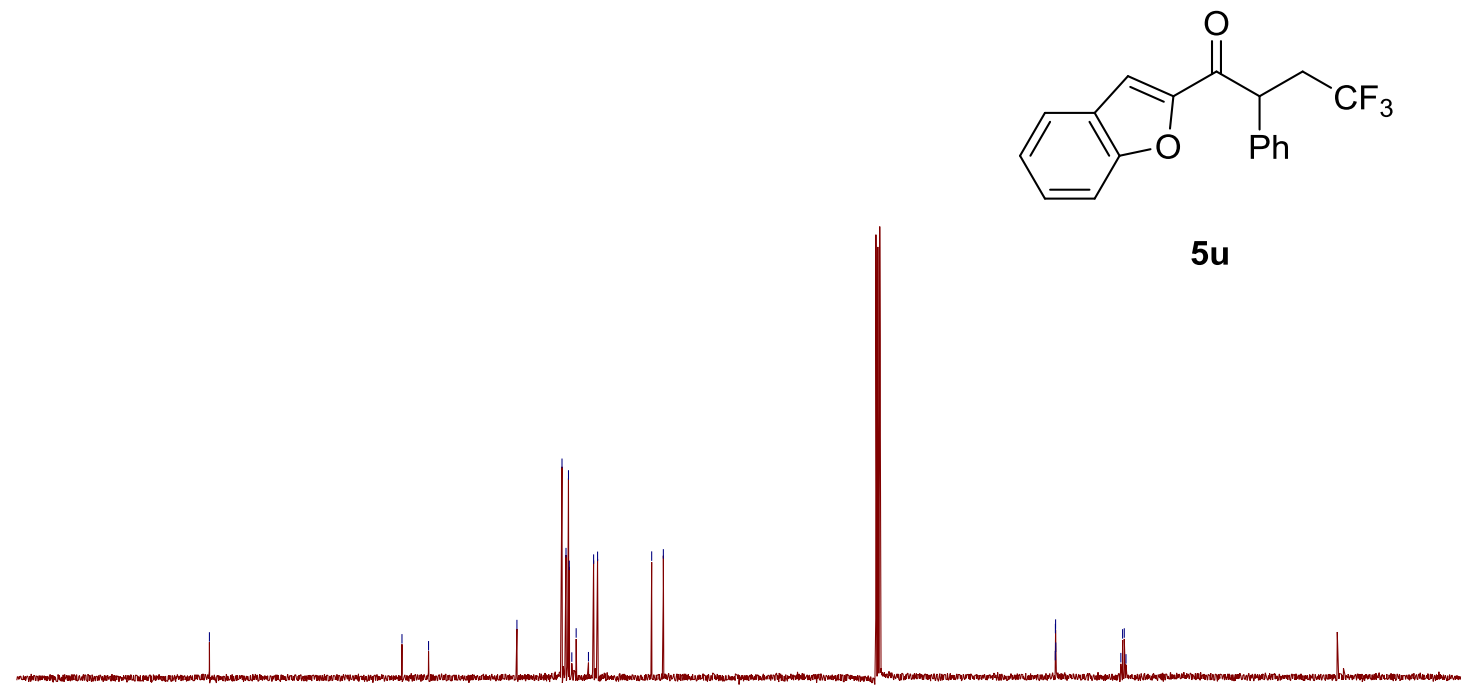


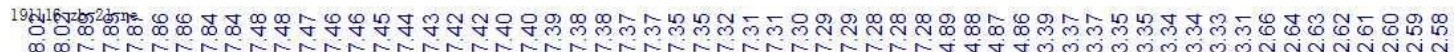

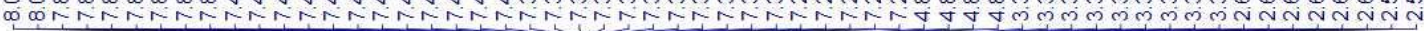
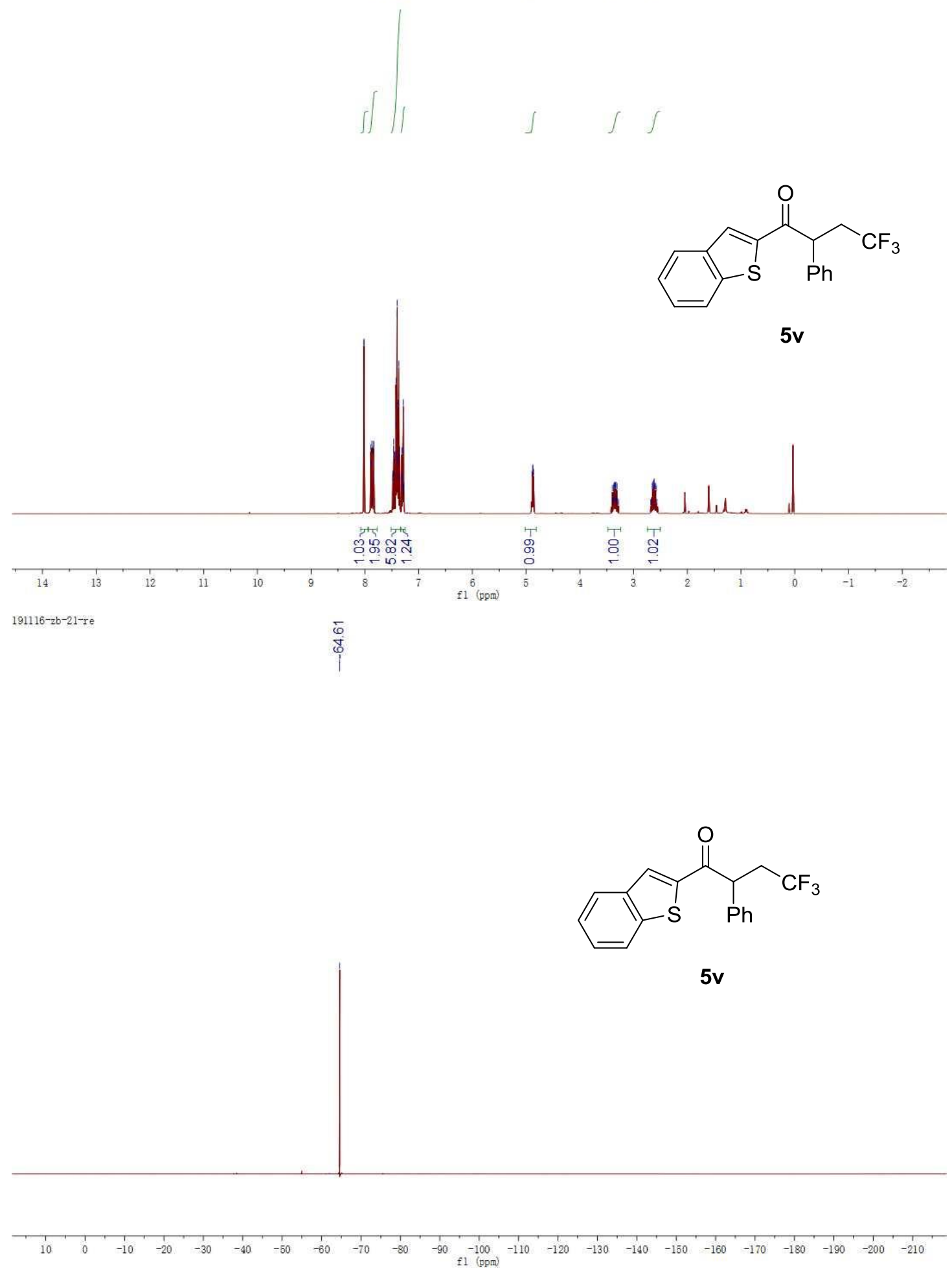

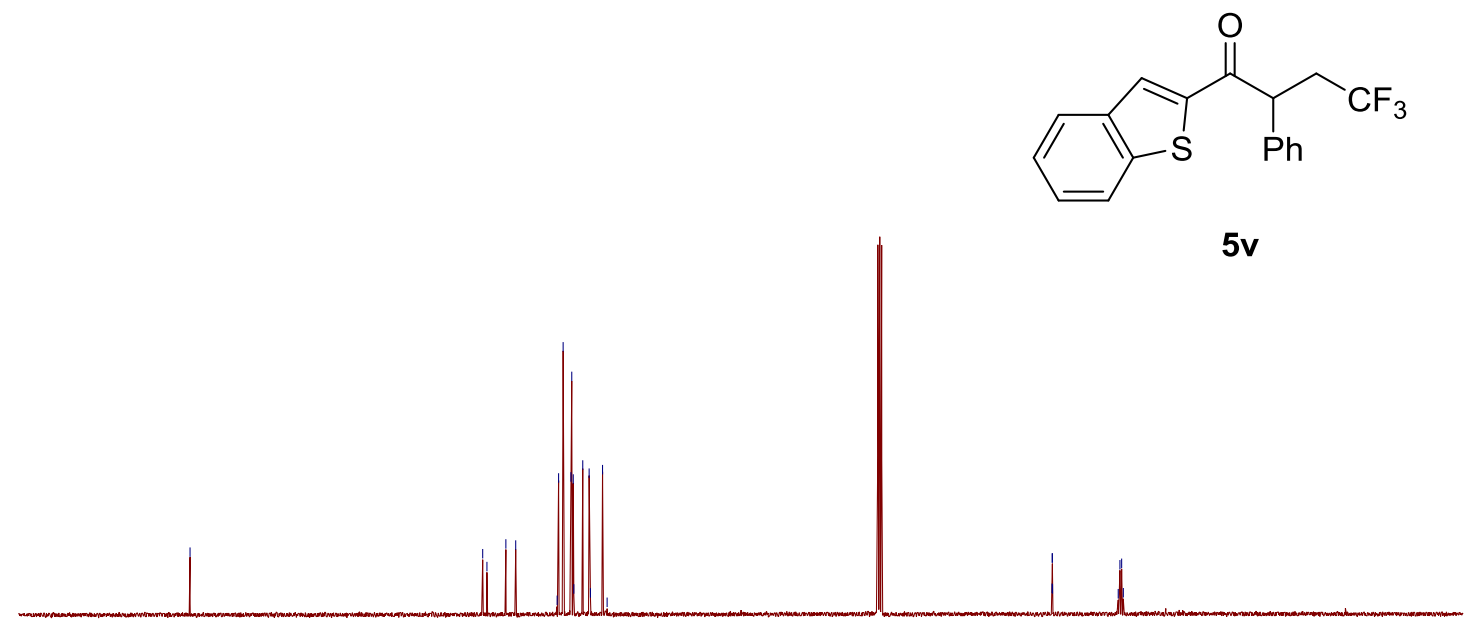

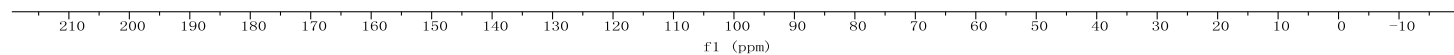

